\title{
Big Data-Driven AI-based Wireless Network Personalization
}

\section{Rawan Alkurd}

A thesis submitted to the Faculty of Graduate and Postdoctoral Affairs in partial fulfillment of the requirements for the degree of

Doctor of Philosophy

in

Electrical and Computer Engineering

Department of Systems and Computer Engineering

Carleton Univeristy

Ottawa, Canada

September 2020

(C) 2020

Rawan Alkurd 


\section{Abstract}

There is a consensus that trends in emerging wireless technologies, such as explosive data requirements and proliferating services and applications, are creating serious issues for the management of user experience. This is due to the fact that, unlike Quality-of-Service (QoS), for which technical metrics are available such as packet error rate, it is difficult to analytically model and measure "user experience" in wireless networks. Besides, current communication networks use design methodologies that prevent the realization of maximum network efficiency. For all these reasons, more agile, intelligent, and flexible networks are required. Such networks should be capable of micro-managing resources in a way that meets each user's service quality expectations while using a minimum amount of resources. This micro-management of network resources has ushered in the concept of wireless network personalization. Personalized networks optimize two correlated and contradicting objectives in real time: user satisfaction and resource utilization. In this thesis, the utilization of Artificial Intelligence (AI), big data analytics, and real-time non-intrusive user feedback is proposed in order to enable the personalization of wireless networks. Based on each user's actual QoS requirements and context, a Multi-Objective Optimization (MOO) formulation enables the network to micro-manage and optimize the provided QoS and user satisfaction levels simultaneously. In addition, to enable user feedback tracking and measurement, user satisfaction is modeled based on the Zone of Tolerance concept. Furthermore, a big data-driven AI-based personalization framework is proposed to integrate personalization into wireless networks. Moreover, a MOO problem is formulated to model the personalized resource allocation problem in wireless networks. Then, to validate the formulated problem, a proof-of-concept prototype is built using the wireless personalization framework in which Evolutionary Multi-Objective Optimization (EMOO) is utilized to find the optimum Pareto front solutions. Finally, since user privacy is a crucial concern, a privacy-preserving framework, which is based on the concept of Differential Privacy, is proposed for personalized wireless networks. 


\section{Acknowledgements}

First and foremost, I can never count the blessings of almighty Allah on me. I am grateful to Allah for all that I am, and all that I have.

Since I embarked on this Ph.D. degree adventure, 5 years ago, many people and things have changed my life. As I approach the end of this journey, I can not help but think how far I have changed and how the difficulties and challenges I have faced made me stronger, wiser, and fearless. This experience taught me to have courage, be brave, have a free well, limitless imagination, and be kind to all people. What strikes me the most when I think back over the past 10 years of my life is how important other people were in being who I am today and in arriving at this stage of my life. Therefore, I would like to thank some of them in the following few lines.

First of all, I would like to express my sincere gratitude to Professor Halim Yanikomeroglu for changing my life by letting me be part of his incredible research team and for giving me the opportunity to work under his supervision. He is my best role model for a smart and quality researcher and visionary. His patience, flexibility, genuine caring and concern, and faith in me enabled me to overcome many of the surrounded difficulties and challenges. I will never forget the great help and time you granted me and I will always remember your kindness and your beautiful motivating words. Thank you for everything.

I will forever be thankful to Dr. Ibrahim AbuAlhaol who literally changed and still changing my life. I still think fondly of my time as an undergraduate student in his classes. Dr. Ibrahim was the reason why I decided to pursue a career in research. He was the reason why I admire wireless communications and AI technologies. Without his guidance and constant feedback, this Ph.D. would not have been achievable. The joy and enthusiasm he has for his research were 
contagious and motivational for me, even during tough times. I owe you Dr. Ibrahim for all the things you did for me. It has been an honour to be your student.

Words cannot express my earnest gratitude towards my father and mother who surrounded me with their warm love and care. My father and mother sacrificed their lives for me and for my brothers and sisters. They have instilled any admirable qualities in me, and given me a solid foundation to confront life. They have taught me about hard work and self-respect, about persistence and being independent. I love them so much, and I would not have made it this far without them. My younger sister Razan has been my best friend throughout my whole life journey. I love her so dearly and thank her for her support: you will always be a special person in my life. I would thank our family's little angel, my youngest sister Alma. She is one of the best things that happened to me in my life. Her beautiful smile and innocent words make me deeply thankful to Allah for blessing us with her presence amongst our family. I also want to thank my three dear brothers Ahmad, Mohammad, and Abdulrahman for their help and support, and for the good times we spent together as one loving family. Thanks to my lovely family: you have always been there for me in every step of my life, and I owe all my success to you.

Finally, this project would not have been completed without the support from the government of Canada, which granted me the Vanier scholarship, Canada's most prestigious scholarship.

Rawan Alkurd 
I dedicate this thesis to

my father, mother, Razan, Alma,

Ahmad, Mohammad, and Abdulrahman

for their constant support and unconditional love.

I love you all dearly. 
"Your time is limited, so don't waste it living someone else's life. Don't be trapped by dogma, which is living with the results of other people's thinking. Don't let the noise of others' opinions drown out your own inner voice. And most important, have the courage to follow your heart and intuition."

Steve Jobs 


\section{Contents}

Abstract

Acknowledgements

List of Figures $\quad$ xii

List of Tables $\quad$ XV

Abbreviations $\quad$ xvi

1 Introduction 1

1.1 Introduction to the Research Problem . . . . . . . . . . . . . 2

1.1.1 Why current wireless networks are not sufficiently efficient? . 2

1.1.2 How to further optimize wireless networks? . . . . . . . . . 2

1.2 Motivation . . . . . . . . . . . . . . . 3

1.2.1 Why does personalization matter?.......... . . 3

1.2.1.1 Not everyone fits the mold . . . . . . . . . . . . . 4

1.2.1.2 Act at the speed of users ............ . . . . . . 4

1.2.1.3 Pricing paradigms . . . . . . . . . . . 4

1.2.2 Benefits of the proposed technology . . . . . . . . . 5

1.2 .3 Commercial value . . . . . . . . . . . . . . . 6

1.3 Aims and Objectives . . . . . . . . . . . . . . 7

1.4 Contributions . . . . . . . . . . . . . . . . 10

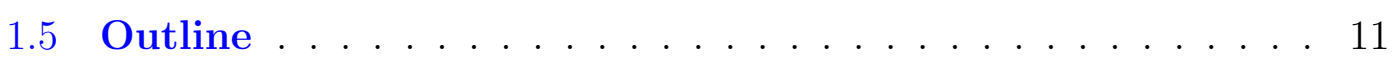

1.6 Publications and Patents . . . . . . . . . . . . . 12

2 Big Data-Driven and AI-based Framework to Enable Personalization in Wireless Networks $\quad 14$

2.1 Background ......................... 15 
2.1.1 The application of ML and big data analytics in wireless communication systems . . . . . . . . . . . 15

2.1.2 Network slicing vs. network personalization . . . . . . . . 18

2.1.2.1 Network slicing in $5 \mathrm{G}$. . . . . . . . . . 18

2.1.2.2 Network slicing vs. network personalization . . . . 20

2.2 Types of Data that Can be Mined to Personalize Cellular

Networks . . . . . . . . . . . . . . . . . . 22

2.2 .1 Network data . . . . . . . . . . . . . . . . . 22

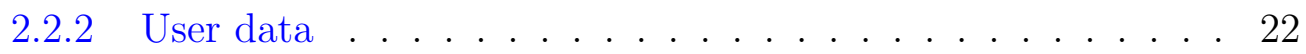

2.2 .3 Other sources of data . . . . . . . . . . . . . . 23

2.3 Big Data-Driven AI-based Framework to Enable Personalization in Wireless Networks . . . . . . . . . . . . 23

2.3 .1 Development . . . . . . . . . . . . . . . 23

2.3.1.1 Data mapping . . . . . . . . . . . 25

2.3.1.2 Data preprocessing . . . . . . . . . . . . 25

2.3.1.3 Cluster users into personas . . . . . . . . . 25

2.3.1.4 Multi-phase persona and user satisfaction prediction 25

2.3.2 Deployment . . . . . . . . . . . . . . . 26

2.3.3 Production . . . . . . . . . . . . . . . 26

2.3.3.1 Data collection and preprocessing . . . . . . . 26

2.3.3.2 Data-driven multi-objective optimization . . . . . . 26

2.3.3.3 Resource allocation . . . . . . . . . . . 26

2.3.3.4 Measure $Q \circ S_{p}$ and get user satisfaction feedback . 27

2.3.3.5 Tuning.................... 27

2.3.3.6 Online learning . . . . . . . . . . . . . . 27

2.4 Illustrating the Benefits of Personalized Wireless Networks 27

2.4.1 Experimental Setup . . . . . . . . . . . . . 28

2.4.1.1 Cellular network environment . . . . . . . . 28

2.4.1.2 Dataset description . . . . . . . . . . . . . . 29

2.4 .2 Experimental Results . . . . . . . . . . . . . 30

3 User Satisfaction in Wireless Networks 32

3.1 Background ...................... 33

3.1 .1 Types of user satisfaction feedback . . . . . . . . . . . . 33

3.2 User Zone of Tolerance Model . . . . . . . . . . . . . . . . . . 34

3.3 Zone of Tolerance in the Context of Personalized Networks 35

3.4 How Can Personalization Potentially Save Resources and

Increase User Satisfaction? . . . . . . . . . . . . . . . . 37

3.5 User Satisfaction Generation Model . . . . . . . . . . . . . . . 38

4 A Synthetic User Behavior Dataset Design for Data-driven AIbased Personalized Wireless Networks 
4.1 Pros and Cons of Utilizing Synthetic Datasets to Study Personalized Networks . . . . . . . . . . . . . . . 43

4.2 Datasets Generation Model : Tree Data Generator . . . . . 43

4.3 Features Description . . . . . . . . . . . . . 45

4.3 .1 Date, time, and day . . . . . . . . . . . . . . . . . 49

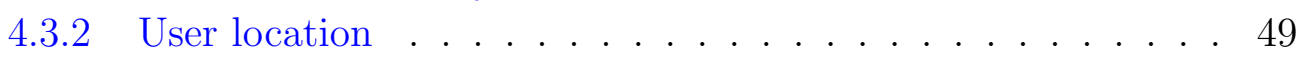

4.3 .3 User speed . . . . . . . . . . . . . . . . . . . . . . 49

4.3 .4 User activity . . . . . . . . . . . . . . . . . . . . . . . . . . 50

4.3 .5 Request arrival time . . . . . . . . . . . . . . . . 50

4.3 .6 Application choice . . . . . . . . . . . . . . . . . . 52

4.3 .7 Service type . . . . . . . . . . . . . . . . . . . . . . . 53

4.3 .8 Demand rate, given rate, and $\Delta \ldots \ldots \ldots$

4.3.9 User satisfaction prediction experiments using the proposed dataset design . . . . . . . . . . . . . . . 55

4.4 Integrating Realistic Characteristics into the Designed Dataset

. . . . . . . . . . . . . . 56

4.4 .1 Clustering users into personas . . . . . . . . . . . 56

4.4.2 Integrating real features from real sensor sata . . . . . . . 56

4.4.3 User satisfaction uncertainty modeling . . . . . . . . . . 58

4.4.4 User satisfaction prediction experiments using the proposed dataset with integrated realistic characteristics . . . . . . 59

$5 \quad$ User Persona Design for Personalized Wireless Networks 63

5.1 Background ..................... 64

5.1 Types of applications utilizing user personas . . . . . . . 66

5.1 .2 User persona design . . . . . . . . . . . . . . . . . 66 66

5.1 .3 Sources of data . . . . . . . . . . . . . . 66

5.1.4 Persona classifications/clustering approaches . . . . . . . . 66

5.2 Benefits of Utilizing User Persona in User Satisfaction Predcition . . . . . . . . . . . . . . 67

5.3 User Persona Design . . . . . . . . . . . . . . . . . . . 67

5.4 Big Data-Driven Persona Prediction Framework for Personalized Wireless Networks . . . . . . . . . . . . 69

5.4.1 Model development . . . . . . . . . . . . . . . . . 69

5.4.1.1 Data collection and labeling . . . . . . . . 69

5.4.1.2 Clustering context-satisfaction user data into personas . . . . . . . . . . . . . . 69

5.4 .1 .3 Data mapping . . . . . . . . . . . . . 71

5.4.1.4 Preprocessing . . . . . . . . . . . . . . 71

5.4.1.5 Train and validate the model . . . . . . . . . 73

5.4.2 Model deployment and production . . . . . . . . . . 74

5.4.2.1 Collect data stack for each user . . . . . . . . . 74 
5.4.2.2 Predict user persona for each data record . . . . . 74

5.4.2.3 Compute confidence . . . . . . . . . . 75

5.4.2.4 Find the predicted persona . . . . . . . . . 75

5.4 .2 .5 Validation ................. 75

5.5 Experimental results . . . . . . . . . . . . . 76

5.5.1 Experimental settings . . . . . . . . . . 76

5.5.1.1 The ML model design . . . . . . . . . . . . 76

5.5.1.2 Cellular network environment. . . . . . . . 77

5.5.2 Experiment 1: Performance of ML models vs. stack size . . 77

5.5.3 Experiment 2: Confidence vs. stack size . . . . . . . . . 79

5.5.4 Experiment 3: Persona in personalized wireless networks . . 80

6 User Satisfaction Prediction in Personalized Wireless Networks 83

6.1 Integrating satisfaction information in wireless networks:

Related work ... . . . . . . . . . . . . . 84

6.2 User Satisfaction Prediction Framework . . . . . . . . . . . 86

6.2.1 Acquire context and KPI data (Block 1 in Fig. 6.2) . . . . . 86

6.2.1.1 Data collection . . . . . . . . . . . . 86

6.2.1.2 Data mapping . . . . . . . . . . . . . 87

6.2.1.3 Data preprocessing . . . . . . . . . . 87

6.2.2 Capture user satisfaction (Block 2 in Fig. 6.2) . . . . . . . . 89

6.2.2.1 Emotions recognition . . . . . . . . . . . 89

6.2.2.2 Predict the correlation to service . . . . . . . . . . 89

6.2.2.3 Predict the actual user satisfaction . . . . . . . . 89

6.2.3 Build the predictive model (Block 3 in Fig. 6.2) . . . . . . . 89

6.2.4 Predict user satisfaction using new unlabeled data (Block 4

in Fig. 6.2) . . . . . . . . . . . . . . . . . . . . . . . . . . 90

6.3 Dataset Analysis and Prepocesssing . . . . . . . . . . . . 90

6.3.1 Dataset filtering and spliting . . . . . . . . . . . . . . 91

6.3.2 Data shuffling and scaling . . . . . . . . . . . . . . . . . . . . . . . . . 92

6.3.3 Encoding categorical values . . . . . . . . . . . . . . . . . . . . 92

6.3.4 Data balancing . . . . . . . . . . . . . . . . . . 92

6.4 Model Structure and Formulation . . . . . . . . . . . . . . 92

6.4.1 Forward propagation ..................... 93

6.4.2 Backwards propagation . . . . . . . . . . . . . . 94

6.5 Experiment Results and Analysis . . . . . . . . . . . 95

6.5.1 Improving the performance of DNN . . . . . . . . . . . 95

6.5.1.1 Number of layers and hidden units . . . . . . . . 95

6.5.1.2 Optimization methods . . . . . . . . . . . 95

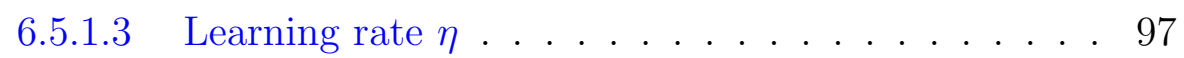

6.5.1.4 Other tuning parameters . . . . . . . . . . 97

6.5.1.5 Performance results . . . . . . . . . . 98 
7 Personalized Resource Allocation in Wireless Networks: An AIEnabled and BD-Driven Multi-Objective Optimization

7.1 Data-Driven Surrogate-Assisted Evolutionary Multi-Objective

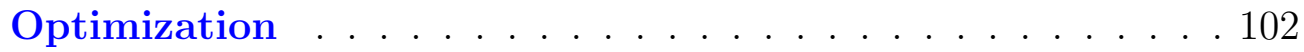

7.1.1 Evolutionary optimization algorithms . . . . . . . . . . 102

7.1.2 Online vs. offline optimization . . . . . . . . . . . . . . . 104

7.1.3 Interactive evolutionary computation . . . . . . . . . . . . 104

7.2 Surrogates in Personalized Wireless Networks . . . . . . . 105

7.2.1 Why we need surrogates? . . . . . . . . . . . . . 105

7.2.1.1 Real-time user satisfaction evalulation . . . . . 106

7.2.1.2 Dynamically evolving user satisfaction models . . . 106

7.2.2 Management of surrogates in personalized networks . . . . . 106

7.2.3 Surrograte model design and performance . . . . . . . . . . 109

7.3 Data-Driven Multi-objective Optimization of Resources in

Personalized Wireless Networks . . . . . . . . . . . 110

7.3.1 Problem description . . . . . . . . . . . . . . . . . 110

7.3.2 Problem formulation . . . . . . . . . . . . . . . . 111

7.3.2.1 System model . . . . . . . . . . . . . . 111

7.3.2.2 Solution encoding: Resource block and power allocation . . . . . . . . . . . . . . . 112

7.3.2.3 Data driven optimization . . . . . . . . . . . . . . . . . . . 112

7.3.2.4 Optimization problem formulation . . . . . . . 112

7.4 EMOO of Resources in Personalized Wireless Networks . . 114

7.4.1 Solution encoding . . . . . . . . . . . . . . . . . . . 114

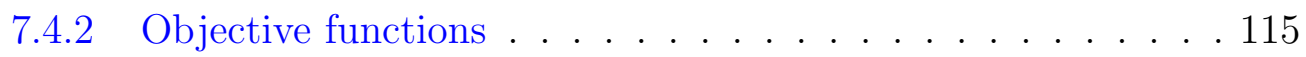

7.4.3 Population intialization . . . . . . . . . . . . . . 115

7.4.4 Selected MOEAs . . . . . . . . . . . . . . . . . . . . . . . . . . . . . . . . . . . . .

7.4.5 Evolutionary operators . . . . . . . . . . . . . . . . . . . . . . . . . . . . . . . . . . . . . .

7.4 .6 Stoping criteria . . . . . . . . . . . . . . 115

7.5 Empirical Analysis and Discussion . . . . . . . . . . 116

7.5.1 Experimental settings . . . . . . . . . . . . 116

7.5.1.1 Cellular network environment . . . . . . . . 116

7.5.1.2 Performance metrics . . . . . . . . . . . . . 117

7.5.1.3 Reference set generation . . . . . . . . . . . 118

7.5.2 Experiment 1: statistical analysis of MOEAs performance results . . . . . . . . . . . . . . . . 120

7.5.2.1 Sample size sufficiency . . . . . . . . . . . . . 121

7.5.2.2 Statistical analysis . . . . . . . . . . . . . . 121

7.5.3 Experiment 2: personalized vs. non-personalized wireless networks . . . . . . . . . . . . . . . . 125

7.5.4 Experiment 3: the impact of uncertainty introduced by surrogates on the performance of MOEAs . . . . . . . . 127 
7.5.5 Experiment 4: scalability Analysis . . . . . . . . . . . . . 129

7.5.5.1 The impact of the number of network users on complexity . . . . . . . . . . . . . . . . . 129

7.5.5.2 The impact of the required solution quality on complexity . . . . . . . . . . . . 130

8 Preserving User Privacy in Personalized Wireless Networks 131

8.1 Differential Privacy . . . . . . . . . . . . . . . . . 133

8.1.1 Definition of differential privacy . . . . . . . . . . . . 133

8.1.2 Central vs. local differential privacy . . . . . . . . . . . 134

8.2 DP-based Privacy-Preserving Framework for Personalized

Wireless Networks . . . . . . . . . . . . . . . . 135

8.2.1 Training at the user edge . . . . . . . . . . . . . 135

8.2.2 Predict user satisfaction in the cloud . . . . . . . . . . . 137

8.3 Empirical Analysis and Discussions . . . . . . . . . . . 138

8.3.1 Dataset and simulation setup . . . . . . . . . . . 138

8.3.2 Experiment 1: The impact of differential privacy on the quality of the user satisfaction predictor . . . . . . . . 138

8.3.3 Experiment 2: The effect of privacy budget and training data size on performance . . . . . . . . . . . . 139

9 Conclusions and Future Research Directions 141

9.1 Synopsis of the Proposed Technology . . . . . . . . . . . . . . 142

9.2 Additional Options and Applications . . . . . . . . . . . . . 146

9.3 Open Research Issues . . . . . . . . . . . . . . . . . . . . . 147

9.3 .1 Dynamic users data . . . . . . . . . . . . . . . . . 147

9.3 .2 Non-intrusive user feedback . . . . . . . . . . . . . . . . 147

9.3.3 User persona clustering . . . . . . . . . . . . . . . . 147

9.3.4 An effective and efficient surrogate management scheme . . . 148

9.3.5 Accurate prediction of user satisfaction . . . . . . . . . . . 148

9.3.6 Advaned IEC MOEAs . . . . . . . . . . . . . . . . . . . 148

9.3.7 Optimizing other aspects of wireless networks . . . . . . . . 149

9.3.8 Data collection, processing, and transmission overhead . . . 149

9.3.9 The correlation of detected user emotions with the provided service quality . . . . . . . . . . . . . . . . . . . 149 


\section{List of Figures}

1.1 Global mobile data traffic and year-on-year growth (EP per month)

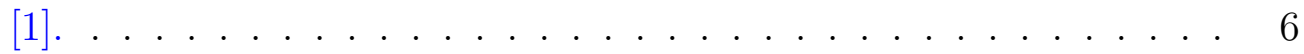

2.1 Comparison between the architecture of network slicing and network personalization. . . . . . . . . . . . . . . 19

2.2 Types of data that can be mined to personalize cellular networks. . 21

2.3 Big data-driven AI-based network personalization framework. . . . 24

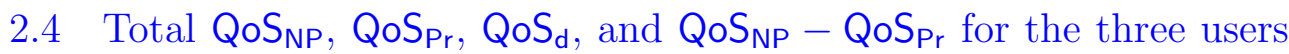
vs. time in hours. . . . . . . . . . . . . . . . . . . . 29

2.5 Average user satisfaction for the three users vs. time in hours for the personalized and non-personalized networks. . . . . . . . . 30

3.1 User Zone of Tolerance (ZoT) model. . . . . . . . . . . . . . . . . 34

3.2 User satisfaction model and a visualization of an example illustrating the relationship between the zone of tolerance, $\Delta$, user satisfaction, and personalized network decisions. . . . . . . . . 36

$3.3 Q_{o} S_{d}, Q_{o} S_{p}, \Delta$, and the corresponding user satisfaction for contexts $C_{1}$ and $C_{2}$ associated with both personalized and non-personalized networks. . . . . . . . . . . . . . . 37

3.4 Satisfaction mapper for $\psi=h$ and $\lambda=0.5,1,2 \ldots \ldots . . . . .39$

3.5 Satisfaction mapper for $\psi=l$ and $\lambda=0.5,1,2$. . . . . . . . . 39

4.1 TG for a working professional persona. . . . . . . . . . . . . . . . 44

4.2 Graphical model representation of hidden Markov model. . . . . . . 45

4.3 Sample instances from the proposed synthetic dataset. . . . . . . . 46

4.4 The relationships between the features: time, location, speed, and activity. . . . . . . . . . . . . . . . . . 4 47

4.5 User speed heat map and the ring of locations for a) a weekday and b) a weekend day. . . . . . . . . . . . . . . . . . 4 48

4.6 The percentage of time the user spent at each location over each time period on a weekday. . . . . . . . . . . . 50

4.7 The histogram of the recorded instances on a weekday for the six activities at two locations. . . . . . . . . . . . 51

4.8 The probability of request arrival (i.e., $P\{N(t, t+\delta)=1\}$ ) vs. $t$ in hours for a weekday. . . . . . . . . . . . . 52 
4.9 The probability of request arrival (i.e., $P\{N(t, t+\delta)=1\})$ vs. $t$ in hours for a weekend day. . . . . . . . . . . . . . 52

4.10 Heat map of the instance count for the requested services and the corresponding applications over a week. . . . . . . . . . 53

4.11 Accuracy swarm plot for 10-folds cross-validation using DT, Knn, and RF algorithms. . . . . . . . . . . . . . 55

4.12 The problem of user satisfaction prediction from user context data. 59

4.13 Satisfaction mapper with added uncertainty for $\psi=h ; \sigma=0.1$, 0.15 , and 0.2 ; and $\lambda=1,2$, and $3 . \ldots \ldots$. . . . . . 60

4.14 Accuracy swarm plot for 10-folds cross-validation using DT, Knn, and $\mathrm{RF}$ algorithms. . . . . . . . . . . . . . . 61

5.1 A comparison between persona design in the literature and the proposed persona design for personalized networks. . . . . . . . . 65

5.2 The proposed big data-driven persona prediction framework for personalized wireless networks. . . . . . . . . . 70

5.3 Cumulative EVR vs. the number of components. . . . . . . . . . 73

5.4 The accuracy and variance of the proposed persona prediction framework vs. stack size using DNN and SVM. . . . . . . . . . 78

5.5 The confidence and confidence variance of the persona prediction framework vs. stack size using DNN. . . . . . . . . . . . . . 79

5.6 Simulation results of the personalized and non-personalized networks. 81

6.1 An example of data mapping for user location feature. . . . . . . . 87

6.2 The proposed big data-driven satisfaction prediction framework. . . 88

6.3 Histogram for user satisfaction classes of the WPP dataset. . . . . 93

6.4 The network structure of the proposed DNN model. . . . . . . . . 94

6.5 Comparison between the accuracies of DNNs using different optimization methods. . . . . . . . . . . . . 96

6.6 Comparison between the training accuracy of DNNs using different learning rates. . . . . . . . . . . . . . . . . . . 97

6.7 Comparison of the DNN model performance for different training data sizes. . . . . . . . . . . . . . . . . . . . . . . . . . . . . 98

6.8 Accuracy swarm plot for 10-folds cross-validation using DT, Knn, RF, and the DNN model. . . . . . . . . . . . . . . . . . 99

7.1 A comparison between data-driven (online) and offline EAs frameworks. . . . . . . . . . . . . . . . . 103

7.2 EMOO management framework to optimize personalized wireless networks. . . . . . . . . . . . . . . . 107

7.3 Inputs and outputs of the Optimum Personalized Resource Allocation (OPA) problem for wireless networks. . . . . . . . . . 110

7.4 The generated approximation sets of the Pareto front solutions by the considered MOEA algorithms. . . . . . . . . . . . . . . . . 119 
7.5 The non-dominated Pareto front reference solution set extracted from the multiple sets generated by the considered MOEA algorithms.120

7.6 Standard Error of the Mean $\left(S E_{M}\right)$ vs. sample sizes. . . . . . . . . 122

7.7 The simulation results of the Direct Feedback Personalized Network (FPN) and the Surrogate-assisted Personalized Network (SPN) for four network users. . . . . . . . . . . . . . . . . . 126

7.8 The average HV computed for different surrogate models with varying performance levels for NSGAII, NSGAIII, SPEA2, and $\varepsilon$-MOEA.128

7.9 The number of users $\mathcal{U}_{b}$ vs. average HV for NSGAII, NSGAIII, SPEA2, and $\varepsilon$-MOEA. . . . . . . . . . . . . . . . 128

7.10 The Number of Function Evaluations (NFEs) vs. average HV for NSGAII, NSGAIII, SPEA2, and $\varepsilon$-MOEA. . . . . . . . . . . . . 129

8.1 Privacy-preserving framework for personalized wireless networks. . 136

8.2 10-folds cross-validation swarm plot for (I) DNN, (II) PCA+DNN, (III) DP-PCA+DNN, (IV) PCA+DP-DNN, and (V) DP-PCA+DPDNN. . . . . . . . . . . . . . . . . . 139

8.3 Accuracy of DP-DNN vs. privacy budgets $\epsilon$ for two different data sizes. . . . . . . . . . . . . . . . . . . . 140 


\section{List of Tables}

2.1 Classification of literature on different applications of machine learning in wireless communication networks. . . . . . . . . . 16

2.2 Simulation parameters. . . . . . . . . . . . . . . . . 28

4.1 The set of services and their associated demand rate for the considered set of applications. . . . . . . . . . . . . . . 54

5.1 Weekdays and weekends location rings for the four user personas. . 68

5.2 Features of the UCS dataset. . . . . . . . . . . . . . . . . . . . . . . . 72

5.3 Cellular network simulation parameters. . . . . . . . . . . . 77

6.1 Features of the WPP dataset. . . . . . . . . . . . . . . 91

7.1 Performance of the adopted DNN design in terms of accuracy. . . . 109

7.2 Cellular network simulation parameters. . . . . . . . . . . . . 117

7.3 Statistical and Friedman test results. . . . . . . . . . . . . . . . . 123

7.4 Pairwise comparison of algorithms rejected by the posthoc tests. . . 124 


\section{Abbreviations}

5G-PPP Fifth Generation Public Private Partnership

AI Artificial Intelligence

AUCED All You Can Eat Data

Adam Adaptive Moment Estimation

API Algorithm Performance Indicator

BW Bandwidth

CH Context-Satisfaction dataset

CV Cross-Validation

D2D Device-to-Device

DT Decision Tree

DNN Deep Neural Network

DL Deep Learning

DF Differential Privacy

EMOO Evolutionary Multi-Objective Optimization

EVR Explained Variance Ratio

EAs Evolutionary Algorithms 
FPN Direct Feedback Personalized Wireless Networks

GPS Global Positioning System

GD Generational Distance

HM HomeMaker

HDFS Hadoop Distributed File System

HS High School Student

HMM Markov Model-based

HUX Half Uniform Crossover

HV Hypervolume

IoT Internet-of-Things

ITU-T International Telecommunication Union

IGD Inverted Generational Distance

KPI Key Performance Indicator

Knn K-nearest neighbor

ML Machine Learning

Mbps Megabits per seconds.

MOO Multi-Objective Optimization

MOEAs Multi-Objective Evolutionary Algorithms.

NFV Network Function Virtualization

NPN Non-Personalized Network

NNs Neural Networks

NS Network Slicing 
NSGA-II Non-dominated Sorting Evolutionary Algorithm II

NFEs Number of Function Evaluations

NGR Overall Non-dominated Vector Generation Ratio

OPA Optimum Personalized Resource Allocation

PCA Principal Component Analysis

QoS Quality-of-Service

QoE Quality-of- Experience

ReLU Rectified Linear Unit function

RF Random Forest

RNN Recurrent Neural Networks

RAN Random-Access Networks

RB Resource Block

SGD Stochastic Gradient Descent

SDN Software-Defined Networks

SP Spacing

SPN Surrogate-assisted Personalized Wireless Networks

SVM Support Vector Machine

SIOAs Swarm Intelligence-based Optimization Algorithms

SNR Signal to Noise Ratio

TS Time Slot

TCO Total Cost of Ownership

TG Tree Data Generator 
US University Student

WPP Working Professional Persona dataset

WD Weekdays

WP Working Professional

ZoT Zone of Tolerance 


\section{Chapter 1}

\section{Introduction}

This chapter describes the tackled research problem and the proposed solution. Besides, the value and benefits of the proposed personalization solution for both operators and users are presented. Also, the aims and objectives along with the contributions of this thesis are highlighted. Lastly, the thesis outline and the list of publications are presented. 


\subsection{Introduction to the Research Problem}

\subsubsection{Why current wireless networks are not sufficiently efficient?}

Current wireless telecommunication networks are designed based on worst-case scenarios, being over-engineered to achieve a minimum Quality-of-Service (QoS) value. However, optimizing the wireless network based on worst-case scenarios decreases its efficacy and efficiency, and does not necessarily achieve the desired user satisfaction levels. The currently operating networks and the published literature do not consider any variation in the degree of user satisfaction and assume that all users will be satisfied with the same service performance level at all times. This is due to the fact that, when such networks were designed, the technology required to enable user satisfaction behavior tracking and estimation in real-time was not mature yet.

While the over-engineered network design is sufficient for current networks, it is considered non-efficient for future networks due to the nature of the expected data demand and requirements. Future networks are expected to support a wider range of applications and use cases, such as vehicular ad-hoc networks and virtual reality applications. Such applications require network services to be delivered with a variety of network performance characteristics (e.g., rate, latency, security, and Quality-of-Experience (QoE)) which raises significant technical challenges for service providers today. In addition, revenues associated with different services and applications will widely vary, which, in effect, will bring new business challenges. Current networks lack flexibility in balancing the implementation of cost-optimized and performance-optimized applications; hence, new technologies and solutions should be proposed to cope with the rising challenges and huge demand.

\subsubsection{How to further optimize wireless networks?}

Continuously advancing technologies have contributed to a surge in data traffic, making user satisfaction the cardinal competitive advantage for all service providers. Besides user satisfaction, service providers try to make the most of the scarce resources available. In order to meet these objectives simultaneously, more agile, intelligent, and flexible networks are required. Such networks should be capable of micro-managing resources in a way that meets each user's expectations of the network while using a minimum amount of resources. This micro-management 
of network resources has ushered in the concept of wireless network personalization. Personalized networks optimize two correlated and contradicting objectives in real-time: user satisfaction and resource utilization.

Naturally, wireless networks produce colossal amounts of data and most of this data is in real-time. A system that is capable of digesting these data to create relevant and meaningful decisions in real-time at the user level using Machine Learning (ML) and big data analytics is the ultimate solution to meeting the aforementioned objectives simultaneously and maximize efficiency. This is due to the fact ML is capable of finding insightful information without being programmed or told what to do; hence, model and solve complex problems that can not be solved using mathematical modeling. We refer to such a system as a big data-driven Artificial Intelligence (AI)-based personalized wireless network. Enabling wireless network awareness of context data and user feedback data and, consequently, enabling wireless network personalization will bring substantial benefits to both users and service providers.

This thesis presents a novel method to utilize context data along with nonintrusive user feedback data to personalize wireless networks. While the proposed personalization concept could potentially be applied to all wireless networks, the discussion in this thesis is specifically focused on wireless cellular networks as a use case.

\subsection{Motivation}

There are numerous advantages of studying and integrating personalization into wireless networks. This section starts with presenting the general motivation behind studying and integrating personalization into wireless networks. Then, the direct benefits of the proposed technology for both operators and users are discussed. Besides, the commercial value of adopting the proposed technology is discussed.

\subsubsection{Why does personalization matter?}

Most service providers are scrambling to increase the average revenue per user and to reduce subscriber churn. Therefore, there is a tremendous need to efficiently utilize scarce resources in order to achieve the ultimate balance between user satisfaction and profit. Personalization is indispensable to achieving this balance and to deliver services profitably to users in a win-win setting. In addition, there are 
several other benefits of integrating personalization into wireless networks, including the following:

\subsubsection{Not everyone fits the mold}

Typically, service providers invest in their networks to acquire more subscribers and increase their revenue. Therefore, they always seek to provide their subscribers with the best service quality. Service quality is defined as a comparison between subscribers' expectations and service performance [2]. Current networks are designed mostly to be a "universal fit," where service providers deliver services with a quality appeal to all types of users. However, user expectations of service quality are not "one size fits all." In practical terms, it is difficult to measure user expectations of service quality for current networks as this depends on numerous dynamic and difficult-to-measure variables. For this reason, service providers overengineer the delivery of services and, as a result, many users end up getting more resources than what would actually satisfy them, while others end up getting less than they expect. The way around this inefficiency is to tailor the network for each user's dynamic and context-dependent needs and expectations. This level of fine-grained network decision optimization will enable service providers to provide personalized, satisfactory services for the majority of users at a minimum cost.

\subsubsection{Act at the speed of users}

Although users could show some patterns in their long-term activity and satisfaction behavior, the real-time (short-term) user expectations and behavior evolve continuously. In order to make personalized decisions and actions dynamically, networks need to decide and act at the speed of users (i.e., in real-time). Personalized networks employ ML and big data analytics, which make real-time network decisions and actions possible through automation. Automation can be achieved by analyzing the enormous amounts of data produced by networks to identify relevant patterns and thereby predict context-dependent user needs and expectations.

\subsubsection{Pricing paradigms}

Currently, tariffs are differentiated based on usage. Wireless network personalization will fit well with the pricing paradigms differentiated according to QoS and user satisfaction levels. In addition, not only are QoS and user satisfaction-based pricing paradigms fairer and more attractive to users, but they will also create 
new business models and revenue opportunities for service providers. Based on the new business models, service providers can trade-off complexity and the required user satisfaction and resource-saving levels in the network. For example, a service provider can offer connectivity plans based on a guaranteed satisfaction level instead of pricing plans based on the amount of data provided.

\subsubsection{Benefits of the proposed technology}

The main advantages of the proposed technology include the following:

- Personalized networks are able to efficiently exploit network resources in order to achieve the ultimate balance between user experience and profit in the form of saved network resources.

- Personalized networks result in better user experience, which yields a winwin situation for both operators and users.

- Personalized networks allow operators to maintain a certain user satisfaction level, and hence maintain their customers' retention by meeting their expectations.

- Personalized networks can enable operators to attract more customers with various service price preferences. In other words, user satisfaction can be personalized to each user based on the service price charged by the operator.

- Personalized networks will give operators the flexibility to choose the most suited operating point depending on many factors.

- Personalized networks can enable better crisis management by allowing operators to move the operating point to lower satisfaction values and utilize resources for more critical applications.

In addition to the above, the personalization technology proposed for wireless networks can be applied to any network with users (e.g., wired network and WiFi). Moreover, it can be applied to other businesses and applications that require user feedback to improve the service. However, for the purpose of this thesis, the features are engineered in the proposed solution to fit wireless networks.

Another important advantage is the flexibility of the proposed technology, where it can be integrated into any wireless network at minimum cost in terms of infrastructure. This is because user satisfaction models are trained at the user edge and 
the personalized network optimization process is envisioned to be performed in the cloud (data centers). However, there may be extra overhead and computation power needed to operate and manage the network. In addition, user feedback should be captured, quantified, and labelled based on the proposed solution. This can be an enabler for various applications, especially telecom applications.

\subsubsection{Commercial value}

Future wireless networks, along with their ability to connect billions of devices with very high QoS, are expected to remain a profitable business. As shown in Fig. 1.1, global mobile traffic continues to grow rapidly and it is expected to further increase in the near future. In order to prepare for this huge expected demand in mobile broadband, there is a need to find ways to optimize network resources efficiently. If network users continue to pay, on average, the same amount for their network subscription, profit will decrease rapidly until the cost per GigaByte (GB) overruns the profit per GB. One important lever to address this and to ensure that mobile broadband remains affordable will be to reduce the Total Cost of Ownership (TCO) per GB.

Two factors are crucial to reducing TCO: production cost per GB and revenue per GB. There are many approaches proposed to reduce production cost per GB,

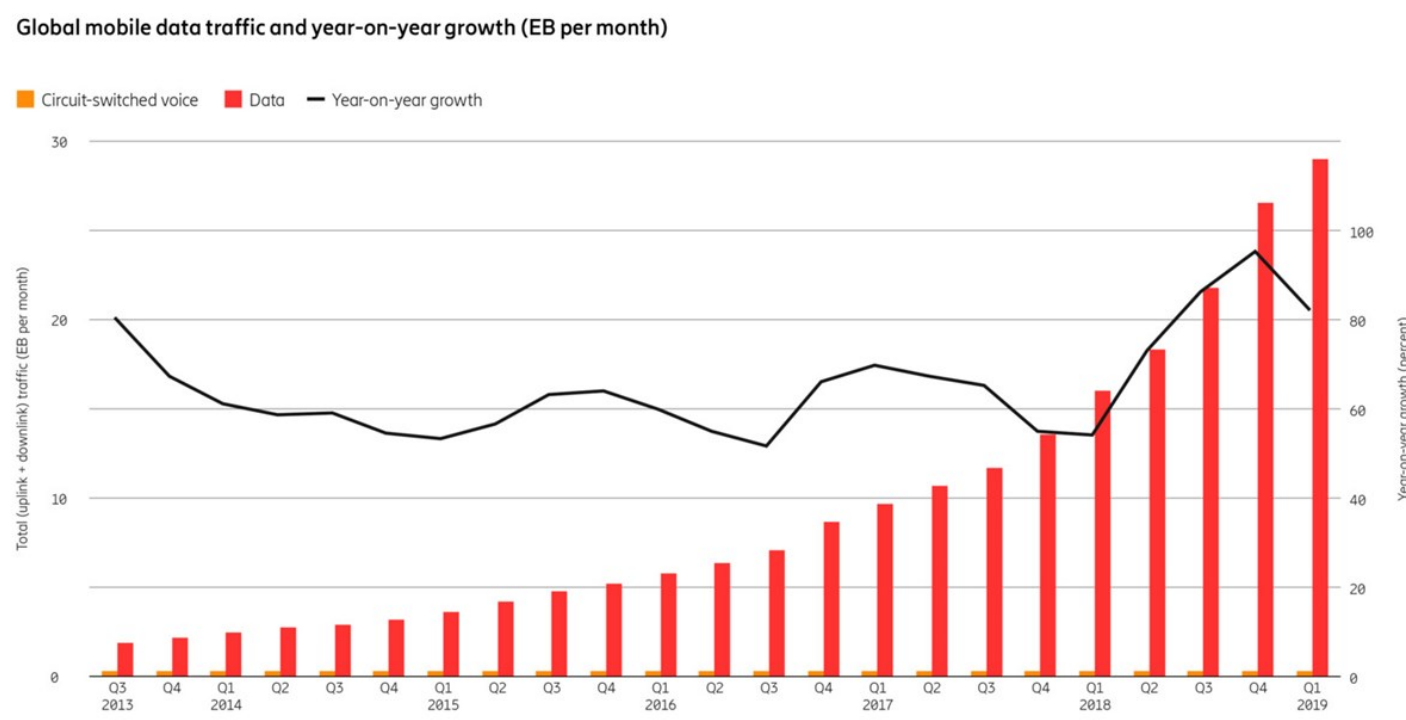

Source: Ericsson traffic measurements (Q1 2019)

FIGURE 1.1: Global mobile data traffic and year-on-year growth (EP per month) [1]. 
such as increasing network energy efficiency [3-5]. Although reducing the production cost per GB is effective, the most powerful way to reduce TCO and to increase profitability is to find new revenue streams in order to increase the revenue per GB. One of the current adopted ways to increase revenue is maximizing network resources utilization through sharing resources in all aspects and dimensions. However, there are further effective ways to empower operators to better monetize their assets and resources. On one hand, the rapidly improving technology could enable operators to adopt new business models with an increased level of granularity. The technology could allow for a more detailed decision-making process to utilize resources in the most cost-effective way. On the other hand, some network subscription plans are described as All You Can Eat Data plans (AUCED) where end users are allowed to use the network as much as they like without the worry of extravagant charges. Such plans are profitable but definitely do not maximize profit. Operators currently adopt a fair resource allocation scheme where the user perception of good QoS is not taken into consideration. Therefore, in addition to benefits discussed in the previous section (Section 1.2.2), network personalization could enable operators to I) adopt micro-plans instead of AUCED plans and II) adopt personalized instead of fair resource allocation schemes. Personalizing the network experience is a key enabler for the adoption of such micro plans.

As explained earlier, current wireless networks are over-provisioned to achieve an unnecessarily high QoS value that would be acceptable to most users. This non-granular, average-based, single-objective approach is currently adopted by all operators. In contrast, the proposed personalization technology provides network operators with improved flexibility of operation in terms of personalized user satisfaction (rather than one averaged satisfaction value good for most users) and the amount of consumed resources.

\subsection{Aims and Objectives}

As explained earlier, further optimization of the resources and increasing network efficiency is needed. The network proposed is more agile and flexible, and is able to micro-manage the resources within a slice and tailor them to the end user's specific needs and requirements. There are two main correlated objectives to be optimized in wireless networks: user satisfaction and resources. These objectives contradict each other and to maximize efficiency, the network should be optimized for both of them. However, since user satisfaction behavior information is not available in current networks, the range of QoS to achieve the required average 
user satisfaction for all users in most situations is predetermined and fixed. Based on the specified QoS range for a particular service, networks are optimized to increase the efficiency of the resources. Nonetheless, this results in an inefficient system. In comparison to current wireless networks, the described personalized networks in this thesis provide a more efficient system, at the same time providing higher levels of user satisfaction.

In addition to the above, although personalized networks offer attractive means of providing better service quality and value for users along with increased profitability for service providers, this win-win situation is however complicated by privacy and security challenges. This due to the fact that personalizing people's experience in networks entails gathering considerable amounts of data about them. For this reason, more research should be conducted to reconcile the goals and techniques used for network personalization with privacy and security restrictions.

The aims of the proposed solution include the following:

- 1) Personalized wireless networks utilize the non-intrusive real-time user satisfaction feedback in order to personalize wireless network decisions and hence, micromanage the available resources so that the required personalized satisfaction levels are achieved with the minimum allocated resources.

- 2) In order to enable the utilization of user feedback (or user satisfaction) to personalize wireless networks, a way to measure and model the non-intrusive real-time user satisfaction feedback should be defined.

- 3) The availability of user behavior and context data is a requirement for the proper operation of the proposed personalized networks. This data can be collected and analyzed or can be obtained from a third party. Since collecting user data from wireless networks for research purposes is difficult (due to confidentiality concerns), another alternative to data collection is synthesizing user data with realistic characteristics.

- 4) Personalized wireless networks are enabled by the real-time analysis of user context data, which raises several privacy concerns. Personalized networks should be realized in a way that does not compromise user privacy and does not risk user sensitive information.

In this thesis, the proposed approach addresses the following objectives:

- Modeling user satisfaction in wireless networks. 
- Modeling/synthesizing a representative data dataset.

- Augmenting and tuning the modeled dataset to be as close as possible to real network data.

- Proposing a way to manage and integrate personalization into current wireless networks.

- Proposing a way to cluster users into personas based on their user satisfaction behavior in order to make the system more efficient and to enable networks to classify and personalize services for new users who do not have a large data history.

- Formulating and solving a data-driven multi-objective optimization problem to maximize user satisfaction levels and minimize utilized resources, simultaneously.

- Proposing a way to realize the benefits of personalized wireless networks without compromising users' private information.

The proposed technology utilizes user satisfaction feedback to personalize the network decisions and hence, micro-manages the available resources so that maximum user satisfaction is achieved with a minimum amount of resources. Saving resources is very valuable to the network since it can be utilized for more critical applications, such as public safety and autonomous cars. In order to achieve the ultimate balance between network resources and user satisfaction, the following questions should be answered:

- Q1: How to integrate personalization into wireless networks?

- Q2: How to measure and quantify user satisfaction in wireless networks?

- Q3: What type of data can be used to personalize wireless networks?

- Q4: How to predict future user satisfaction values?

- Q5: How to optimize resources to achieve the required satisfaction levels using minimum resources?

- Q6: How to maintain the privacy of users in personalized wireless networks? 


\subsection{Contributions}

One aspect of the proposed technology avoids network over-provisioning by designing orthogonal networks that achieve the required user satisfaction levels using minimum resources, such as bandwidth and power. In this thesis, personalization is done using the proposed big data-driven personalization framework. The framework implements a multi-phase persona and user satisfaction prediction. Then, the proposed framework utilizes the final trained model in order to optimize resources in a multi-objective manner; hence achieve the required user satisfaction using minimum resources. The contributions of this work are summarized as follows:

- A framework proposed to integrate personalization into wireless networks.

- A novel Zone-of-Tolerance (ZoT) based model proposed to quantify user satisfaction in wireless networks.

- A synthetic dataset design that mimics real data with realistic characteristics proposed to be used for personalized wireless networks.

- The concept of user persona design in wireless networks proposed to minimize data required to predict satisfaction levels for each user and increase prediction accuracy.

- A user satisfaction and persona prediction frameworks proposed to enable user satisfaction and persona prediction in real-time.

- A formulation for the resource allocation problem as a data-driven MultiObjective Optimization (MOO) decision proposed to enable networks to micro-manage and optimize resources, and users' satisfaction simultaneously based on each user's QoS requirement and user satisfaction behavior.

- A prototype to validate the proposed personalization framework and the proposed MOO formulation in which evolutionary optimization is used to solve the optimization problem.

- A privacy-preserving framework based on the concept of Differential Privacy (DF) proposed to maintain user privacy in personalized wireless networks. 


\subsection{Outline}

The description of the technology in this document is set out in eight chapters as follows:

- Chapter 2 presents background on the types of ML applications in wireless networks and a comparison between network slicing and personalization. Also, this chapter presents the proposed big data-driven AI-based wireless network personalization framework.

- Chapter 3 presents background on user satisfaction prediction in the literature. Besides, this chapter introduces the proposed ZoT model, which is used to quantify user satisfaction in wireless networks.

- Chapter 4 presents the proposed synthetic dataset design for personalized wireless networks. In addition, data analysis and exploration is conducted to verify the required characteristics and attributes embedded within the proposed datasets.

- Chapter 5 presents the concept of persona in personalized networks and provides some background on the utilization of user persona in various applications. Besides, this chapter presents the proposed big data-driven persona prediction framework used to associate a persona type for each user in personalized wireless networks.

- Chapter 6 presents a big data-driven user satisfaction prediction framework in personalized networks. To illustrate the benefits of the proposed framework, it is implemented using deep neural networks as an exemplary ML model used for future user satisfaction prediction in personalized networks.

- Chapter 7 presents a MOO formulation to model the personalized resource allocation problem in wireless networks. Besides, a proof-of-concept prototype is built to solve the formulated problem and to simulate the proposed wireless personalization framework.

- Chapter 8 presents a privacy-preserving framework for personalized wireless networks. The framework utilizes the concept of DF and it is supported by a multi-stage prediction process to protect users' sensitive data.

- Finally, conclusions and a summary of the proposed technology are presented in Chapter 9. Moreover, the chapter lists a number of possible extensions and relevant future research areas that are beyond the scope of this thesis. 
Fig. 2.3 in Chapter 2 illustrates the proposed personalization framework and all it's constituent parts. This work addresses the framework parts required to enable wireless network personalization. The framework parts described in Fig. 2.3 are labeled with the chapters numbers where they are addressed.

\subsection{Publications and Patents}

- R. Alkurd, I. Abualhaol, and H. Yanikomeroglu, "Personalized Resource Allocation in Wireless Networks: An AI-Enabled and Big Data-Driven MultiObjective Optimization," US provisional patent application no: 63/057,560, application date: 28 July 2020.

- R. Alkurd, I. Abualhaol, and H. Yanikomeroglu, "Preserving User Privacy in Personalized Networks," submitted to IEEE Wireless Communications Letters, July 2020.

- R. Alkurd, I. Abualhaol, and H. Yanikomeroglu, "Personalized Resource Allocation in Wireless Networks: An AI-Enabled and Big Data-Driven MultiObjective Optimization," IEEE Access, vol. 8, pp. 144592-144609, 2020.

- R. Alkurd, I. Abualhaol, and H. Yanikomeroglu, "Big Data-Driven AI-based Framework to Enable Personalization in Wireless Networks," IEEE Communications Magazine, vol. 58, no. 3, pp. 18-24, March 2020.

- R. Alkurd, I. Abualhaol, and H. Yanikomeroglu, "User Satisfaction Prediction Framework for Personalized Wireless Networks: A DNN Approach," submitted to 2020 IEEE International Conference on Communications (ICC).

- R. Alkurd, I. Abualhaol, and H. Yanikomeroglu, "Enabling Wireless Network Personalization using Zone of Tolerance Modeling and Predictive Analytics", W.O. Pat. Ser. No. PCT/CA2019/051197, application date: 29 August 2019, publication no: WO2020/041883 A1, publication date: 05 March 2020.

- R. Alkurd, I. Abualhaol, and H. Yanikomeroglu, "User Persona in Personalized Wireless Networks: A Big Data-Driven Prediction Framework," in Proc. 2020 IEEE 92nd Vehicular Technology Conference (VTC-Fall), October 2020 .

- R. Alkurd, I. Abualhaol, and H. Yanikomeroglu, "A Synthetic Dataset Modeling for Data-Driven AI-Based Personalized Wireless Networks," in Proc. 
2019 IEEE International Conference on Communications Workshops (ICC Workshops), May 2019.

- R. Alkurd, I. Abualhaol, and H. Yanikomeroglu, "Dataset Modeling for Data-Driven AI-based Personalized Wireless Networks," in Proc. 2019 IEEE International Conference on Communications (ICC), May 2019. 


\section{Chapter 2}

\section{Big Data-Driven and AI-based Framework to Enable Personalization in Wireless Networks}

In this chapter, the utilization of AI, big data analytics, and real-time non-intrusive user feedback is proposed for enabling the personalization of wireless networks. In order to integrate personalization into wireless networks, a big data-driven AI-based personalization framework is proposed. The first part of the chapter presents background on the applications of $M L$ and big data analytics in wireless communication systems. Then, a comparison between network slicing and the proposed network personalization concept is presented. Lastly, the proposed framework is discussed after which the key concepts of network personalization are demonstrated through a case study. The case study shows how personalization can be realized to enable the efficient optimization of network resources such that a certain level of user satisfaction and revenue (in the form of saved resources) requirements are achieved. 


\subsection{Background}

\subsubsection{The application of ML and big data analytics in wire- less communication systems}

Recently, the application of ML and data analytics in wireless networks has gained a lot of interest within the research communities and the industry. Table 2.1 presents a summary of related literature. In literature, one of the main applications that implement ML in wireless communication is Device-to-Device (D2D) communications. A lot of research has been conducted to utilize ML for solving two main problems: resource allocation and eNB allocation. Mainly, two ML algorithms were adopted: Q-learning and Reinforcement learning. In [6], the authors develop a fully autonomous multi-agent Q-learning algorithm that enables each D2D pair to jointly select the wireless channel and power level to maximize rewards, whereas in [7] the authors utilize Q-learning to solve the relay selection problem in relay-aided D2D networks. Also, the authors in [8] formulate a Bayesian coalition formation game to solve the problem of distributed resource sharing in a D2D enabled heterogeneous network where device pairs choose their transmission channels, modes, base stations, and power levels based only on the locally-observable information. Furthermore, the work in [9] proposes a cooperative reinforcement learning algorithm for adaptive power allocation in D2D communication. Also, the authors in [10] formulate a joint resource block scheduling and power control scheme as multi-agent reinforcement learning using a Markov decision process. In addition to resource allocation, the work in [11] formulates the eNB selection problem as a Markov decision process as well. In order to obtain the network parameters, the authors in [11] also propose a reinforcement learning algorithm that leverages both present conditions and expected future demands.

Another wireless communication application that utilizes ML is Internet-ofThings (IoT). The authors in [12] model both network and users QoE and proposed an allocation algorithm based on Deep Reinforcement Learning to improve the accuracy of QoE evaluation adaptively. In addition, the work in [13] proposes the prediction of IoT traffic using Q-learning.

A large body of literature considers the application of ML in cellular networks. The first problem tackled using ML in cellular networks is interference mitigation. For instance, the authors in [14] propose a self-organized power allocation technique to solve the interference problem caused by a femtocell network operating on the same channel as an OFDMA cellular network. Moreover, the authors in [15] 
TABLE 2.1: Classification of literature on different applications of machine learning in wireless communication networks.

\begin{tabular}{|c|c|c|c|c|c|}
\hline Ref. No. & \multicolumn{2}{|c|}{ Application } & \multicolumn{2}{|r|}{ Algorithm } & Objective \\
\hline$[6,7]$ & \multirow{4}{*}{\multicolumn{2}{|c|}{ Device-to-Device networks }} & \multirow{4}{*}{ Reinforcement Learning } & Q-learning & \multirow{3}{*}{ Resource allocation } \\
\hline$[8]$ & & & & Bayesian & \\
\hline$[9]$ & & & & $\overline{\text { Cooperative Reinforcement Learning }}$ & \\
\hline$[10,11]$ & & & & Markov Decision Process & eNB selection/Resource allocation \\
\hline$[12,13]$ & \multicolumn{2}{|c|}{ Internet of things } & \multicolumn{2}{|c|}{ Reinforcement Learning/Q-learning } & Resource allocation/Traffic prediction \\
\hline$[14]$ & \multirow{6}{*}{ Cellular network (LTE) } & Femto cells & \multicolumn{2}{|c|}{ Q-learning } & Inter-cell interference coordination \\
\hline$[16]$ & & Pico cells & \multirow{3}{*}{\multicolumn{2}{|c|}{ Reinforcement Learning }} & \multirow[b]{2}{*}{ Resource allocation } \\
\hline$[17,18]$ & & HetNets & & & \\
\hline [19] & & Self healing & & & Cell outage compensation \\
\hline$[20]$ & & Massive MIMO & Bayesian Learning & Gaussian Mixture & Channel estimation \\
\hline$[15]$ & & Self-Organizing Networks & Reinforcement Learning & Cooperative Reinforcement Learning & Inter-cell interference coordination \\
\hline$[21]$ & \multicolumn{2}{|c|}{ LTE-U and WiFi } & \multirow{2}{*}{\multicolumn{2}{|c|}{ Reinforcement Learning }} & \multirow[b]{2}{*}{ Resource allocation } \\
\hline$[22,23]$ & Green wire & less networks & & & \\
\hline$[24]$ & \multirow{2}{*}{\multicolumn{2}{|c|}{ Cognitive radio }} & \multirow{2}{*}{ Bayesian Learning } & Hidden Markov Model & Estimation of primary user parameters \\
\hline$[25,26]$ & & & & - & Cooperative spectrum sensing \\
\hline
\end{tabular}


use Cooperative Reinforcement Learning with a Fuzzy Q-Learning implementation to deal with the Inter-Cell Interference Coordination problem in the downlink of cellular OFDMA (LTE and WiMAX) systems in the context of Self-Organizing Networks. The second problem discussed in the literature is resource allocation in cellular networks. The authors in [16] utilize reinforcement learning to propose a resource sharing algorithm that manages interference in LTE Pico Cells. Another example is the work in [17] where the authors propose a resource allocation policy for HetNets in order to maximize the energy efficiency of the overall network. In addition, using reinforcement learning for Uplink/Downlink resource allocation in 5G HetNet is discussed in [18]. The third problem in literature tackled using ML in the cellular networks is cell outage compensation. The work in [19] presents an automatic and self-organized Reinforcement Learning-based approach for cell outage compensation which controls the inter-cell interference generated at the borders of the extended cells. The fourth problem discussed in the literature is channel estimation. For example, in [20], the authors use a Bayesian learning method to estimate channel parameters of the considered links.

In addition to cellular networks, research problems related to LTE-U and WiFi are also approached using ML and data analytics. For example, the authors in [21] propose a scheme that uses Q-learning algorithms to dynamically allocate blank subframes so that both LTE-U and WiFi systems can successfully coexist. Furthermore, ML is applied to green heterogeneous wireless network problems. For instance, the authors in [22] propose a framework to improve the performance of green heterogeneous wireless networks where a deep reinforcement learning approach is adopted to solve the resource allocation problem. In addition, traffic offloading and power allocation for green HetNets is tackled in [23] using reinforcement learning.

Cognitive radio is one of the important applications of ML in wireless networks. In literature, ML is utilized to tackle two main cognitive radio network problems: estimation of primary user parameters and spectrum sensing. The authors in [24] model a cognitive radio system as a hidden Markov model where the channel parameters are estimated by the standard expectation-maximization algorithm. Moreover, the authors in [25] propose a cooperative spectrum sensing scheme based on the expectation-maximization algorithm. Finally, Bayesian learning is used in [26] to proposed a rate selection scheme over millimetre-wave channels in cognitive radio networks. 
In contrast to all of the literature work discussed in this section, the proposed technology in this thesis applies ML and big data analytics to estimate user satisfaction behavior in different contexts. Based on the estimated user satisfaction behavior, a framework to micro-manage and personalize decision-making in wireless networks is proposed such that a certain goal in the network is achieved.

\subsubsection{Network slicing vs. network personalization}

There are some similarities between the concept of Network Slicing (NS) and network personalization; therefore, this section provides some background on NS in 5G. Then, the discussion progresses towards distinguishing between NS and network personalization in terms of functions and requirements.

\subsubsection{Network slicing in 5G}

Network Function Virtualization (NFV) technology is proposed for 5G to isolate the software and hardware aspects of networks in order to transform network functions from dedicated hardware appliances into software-based applications. Along with NFV, Software-Defined Networks (SDN) are considered to be enablers for NS in 5G [27]. The concept of NS is proposed to allow operators to provide customized, reliable services with increased efficiency while reducing capital expenditure and operating expenses of wireless networks [28-31]. Each slice is associated with a set of resources, including bandwidth and network topology [32]. What distinguishes $5 \mathrm{G}$ NS from current QoS-based solutions is its ability to provide an end-to-end virtual network tailored to application requirements. This will enable service providers to deploy only the functions necessary to support a certain application. Hence, service providers would not need to deploy full functionalities to support applications that utilize a small part of those deployed functionalities [33].

\section{- Use cases}

ITU and Fifth Generation Public-Private Partnership (5G-PPP) have identified three main use case categories of NS: enhanced mobile broadband, massive machinetype communications, and critical communications [33]. Within each category, more specific use cases can be defined. Each use case has a set of heterogeneous requirements that can not be satisfied by the current one-size-fits-all architecture of current networks.

\section{- Network slicing architicture}

In literature, the research and various proposals on 5G NS revolve around three 


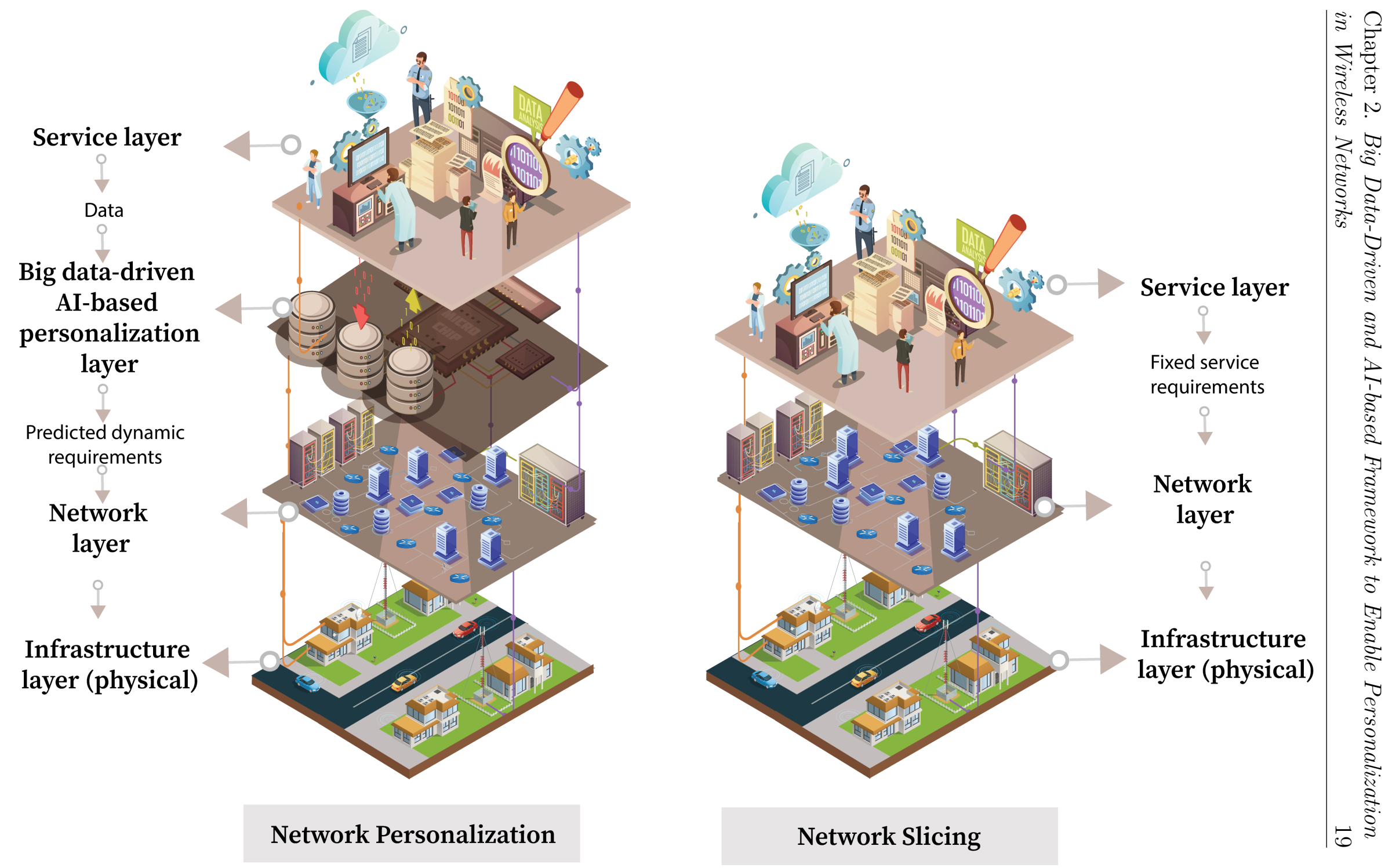

FIGURE 2.1: Comparison between the architecture of network slicing and network personalization. 
main layers: infrastructure layer, network function layer, and the service layer [30]. As shown in Fig. 2.1, the infrastructure layer refers to the network physical layer including Random-Access Networks (RAN) and core networks. Also, the infrastructure layer in NS is responsible for resource management and allocation of resources to slices. The second layer is the network function layer. This layer is responsible for managing all the operations of the network functions that are virtually placed and chained. These chained virtual networks offer end-to-end services that meet certain requirements and constraints. These requirements and constraints are defined and managed by the service layer, shown in Fig. 2.1. The service layer is considered the most important layer as it is responsible for linking the service business model with the appropriate network functions and infrastructure. As shown in Fig. 2.1, the process of defining the service type associated with a network slice and its description and requirements is done in the service layer. In the literature, researchers proposed two approaches to describe service requirements. The first approach describes services with a set of traffic requirements, such as throughput and latency. On the other hand, the second approach suggests describing the services with more details in terms of the specific functions and technologies that will guarantee the fulfillment of the requirements.

\subsubsection{Network slicing vs. network personalization}

What distinguishes 5G NS from the current QoS-based solutions is its ability to provide an end-to-end virtual network tailored to application requirements. Although NS contributes to the improvement of network efficiency, further optimization of the resources and increasing the network efficiency is needed; hence, an even more agile and flexible network is required. As shown in Fig. 2.1, personalized networks advocated in this thesis are not only tailored to application requirements but also to the dynamic user demands and expectations within the application. In other words, instead of specifying a fixed set of quality requirements, personalized networks are supported with an intelligent big data-driven layer to decide on the dynamically evolving user requirements. This enables the service providers to provide the necessary service quality required to achieve the target user satisfaction level for each user and any application. 


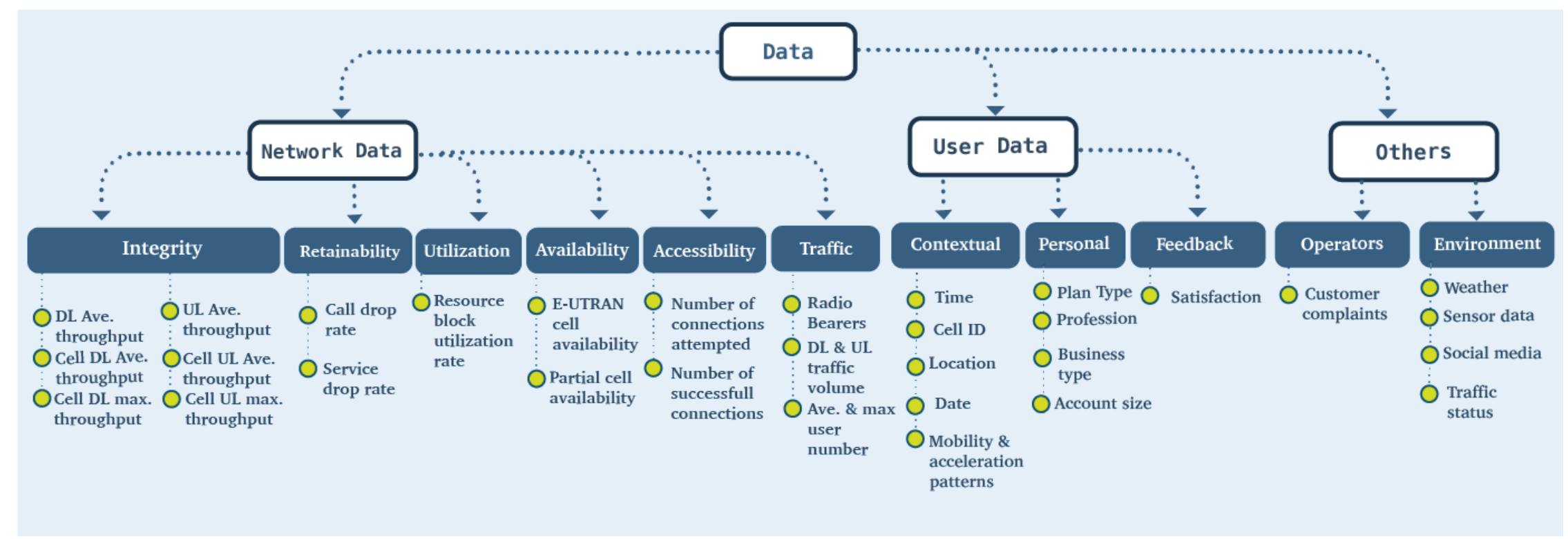

Figure 2.2: Types of data that can be mined to personalize cellular networks. 


\subsection{Types of Data that Can be Mined to Per- sonalize Cellular Networks}

Many variables can affect users' behavior and their perception of service quality. In [34], the authors discuss the different factors that could contribute to the model of QoE. Along with the widely varying users' satisfaction behavior, there are millions of users and devices connected to the network, which adds to the complexity and dimensionality of the problem. Besides, data collected from communication networks are massive, complex, unstructured, and increase in three dimensions: volume, velocity, and veracity. The problem of extracting knowledge from this huge amount of data is divided into two subproblems: a big data problem, and a modeling and prediction problem. In Fig. 2.2, the fundamental aspects and sources of data in personalized cellular networks are described.

\subsubsection{Network data}

As shown in Fig. 2.2, the first type of data is network data. Cellular networks produce an enormous amount of data with different types and volumes. However, to solve the personalization problem, certain types of data are more relevant, such as data describing the network performance over different links and sessions (i.e., Key Performance Indicators (KPIs), see Fig. 2.2).

\subsubsection{User data}

Another important piece of data for personalized cellular networks is user data. As shown in Fig. 2.2, user data is categorized into three categories; contextual, personal, and feedback data. Contextual data defines the context and enables user behavior modeling and prediction. Fig. 2.2 lists a set of exemplary contextual user data such as time, location, and mobility patterns. The second category of user data is personal data, which cannot be collected directly through the network. These data are often collected from customers when they first subscribe to the service and after signing a disclosure or privacy agreement. Fig. 2.2 identifies six types of personal data such as business and plan type. As shown in Fig. 2.2, the third category of user data is user satisfaction feedback. User satisfaction feedback is sent back to the cloud to use it for validating and improving the prediction model.

The process of measuring user satisfaction could be done in real-time or offline and in a number of intrusive and non-intrusive manners. Intrusive feedback data 
Chapter 2. Big Data-Driven and AI-based Framework to Enable Personalization in Wireless Networks

include user complaint data and real-time user feedback. However, non-intrusive feedback data include measurements from acceleration, voice, and camera sensors. Using AI, real-time non-intrusive feedback data can be utilized to predict user satisfaction feedback. In order to practically implement the proposed system, the network should depend mainly on non-intrusive real-time user feedback.

\subsubsection{Other sources of data}

The third category identifies data available from other sources such as social media, weather status, traffic status, and sensor data. Usually, these types of data are unstructured and need pre-processing before being ingested by the prediction model.

\subsection{Big Data-Driven AI-based Framework to En- able Personalization in Wireless Networks}

Data collected from communication networks are massive, complex, unstructured, and increase in three dimensions: volume, velocity, and veracity. The problem of extracting knowledge from this huge amount of data presents two subproblems: a big data problem and an AI problem. AI is defined as any process that senses the environment and takes actions to maximize the success probability of the targeted goal. Until recently, it has not been feasible to solve such a problem in real-time. However, computing power and distributed computing technologies are improving rapidly to the point that the use of big data analytics and prediction techniques for practical near-real-time applications (e.g., processing sensor data) are currently possible.

As shown in Fig. 2.3, the proposed personalization framework collects information from the user environment and the network, predicts user needs and tolerance to service quality, and optimizes resource allocation to minimize cost and maintain certain user satisfaction levels. The proposed framework consists of three stages:

\subsubsection{Development}

The development stage is composed of the following modules all of which are implemented offline: 


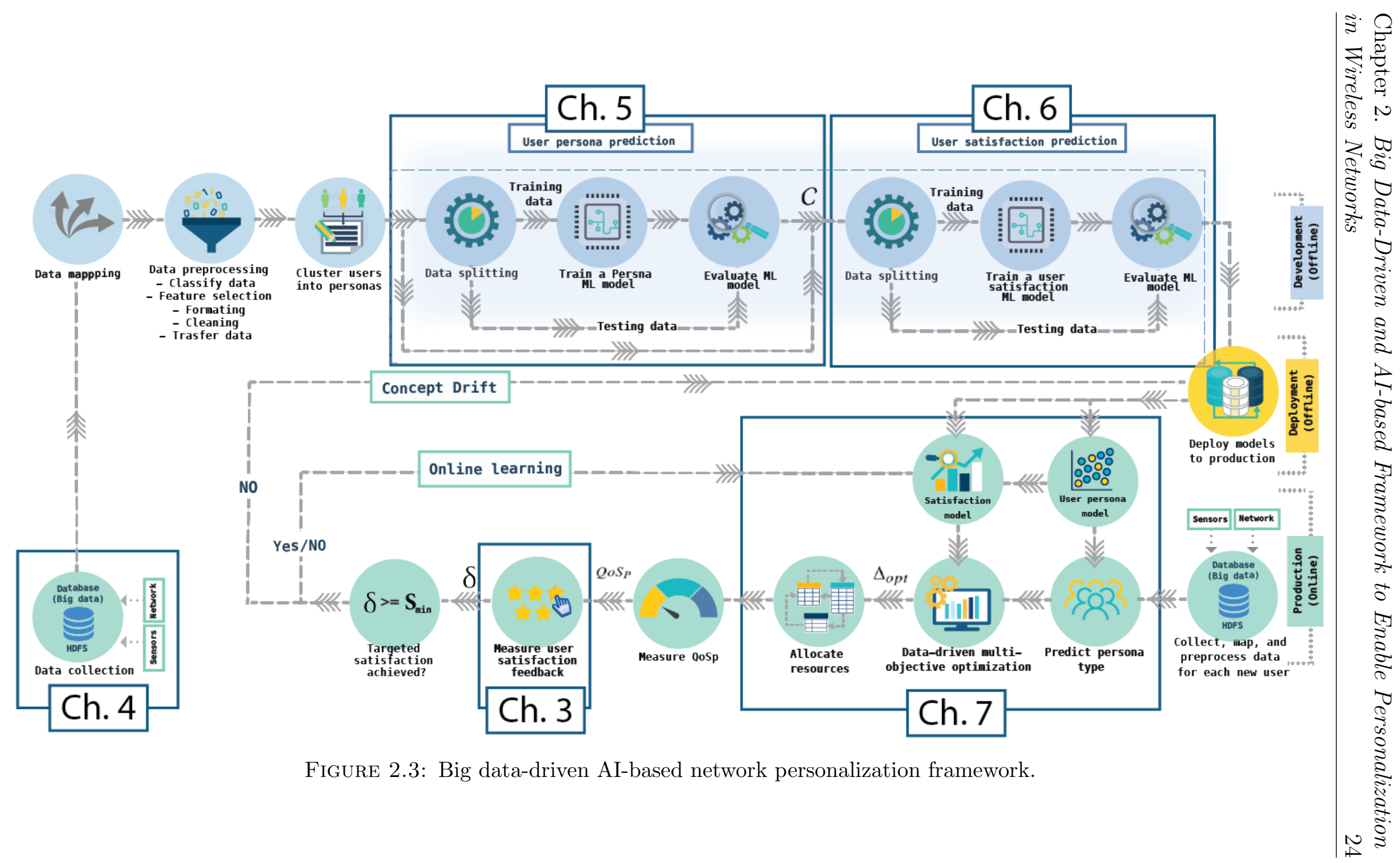




\subsubsection{Data mapping}

Data from different users are mapped to shared space. Mapping user data is an essential step as it enables ML models to capture correlations and inherent patterns. For instance, user location is recorded as Global Positioning System (GPS) coordinates. However, generally, user satisfaction behavior is actually correlated to a particular type of location (e.g., home) rather than GPS coordinates.

\subsubsection{Data preprocessing}

Retransform the dataset and extract useful features. This step is important because it contributes to the reduction of noise and irrelevant data, which can degrade the predictive model's performance.

\subsubsection{Cluster users into personas}

A group of users who share similar user behavior and satisfaction patterns is referred to as a persona [35]. Associating users with pre-existing user personas will enable networks to provide highly personalized services with a minimal amount of data. Besides, the utilization of federated learning can enable different users to share the same persona type to learn a shared model while keeping all the training data on their devices. This can reduce the required communication overhead and minimize the privacy concerns associated with transferring user data over the network.

\subsubsection{Multi-phase persona and user satisfaction prediction}

At this point, the network has access to labeled context data with user persona and satisfaction levels. The processed data is used to build an ML model to predict user satisfaction levels for each user. As shown in Fig. 2.3, the user satisfaction prediction problem is approached using a two-phase ML model. The first phase is designed to output the personas probability vector $\mathcal{C}$. In Chapter 5 , a workflow to implement the persona prediction phase and compute $\mathcal{C}$ is proposed [36]. The second phase digests $\mathcal{C}$ along with the preprocessed labeled data in order to build a model capable of predicting the user satisfaction levels, for new and existing users, using a minimum amount of data. In Chapter 6, a deep neural network model to predict user satisfaction in wireless networks is proposed [37]. 


\subsubsection{Deployment}

At the deployment stage, the output ML model from the previous stage is integrated into the production environment to start making practical decisions based on new data. There are several methods used to deploy ML models. In the proposed framework, to automate the prediction process, the ML model is deployed as an online ML model. Online ML models continue to update and train as more data becomes available to the network.

\subsubsection{Production}

The production stage is where the network utilizes the trained ML models to achieve network personalization in real-time. The production stage is composed of the following modules:

\subsubsection{Data collection and preprocessing}

The first step is to continuously collect context information from users to predict personalized user satisfaction behavior. Data are collected from different sources, such as sensors and network data, and stored in a big data database (e.g., Hadoop Distributed File System (HDFS) [38]). Then, data are preprocessed using the same workflow used in the development stage.

\subsubsection{Data-driven multi-objective optimization}

The next step is to use the multi-phase ML model trained in the development stage as an input to a multi-objective optimization problem. The optimization problem is formulated to micro-manage and optimize resources, and users' satisfaction simultaneously based on each user's QoS requirement and user satisfaction behavior. This optimization problem utilizes the ML model as its fitness function. Moreover, the targeted satisfaction level for each user is decided by the network service provider and fed as an input to the multi-objective optimization problem. The optimization problem outputs the optimum choice of $\Delta\left(\Delta_{o p t}\right)$ which achieves the required satisfaction level using the minimum amount of resources.

\subsubsection{Resource allocation}

The network utilizes $\Delta_{o p t}$ and other network parameters to allocate the best Resource Blocks (RBs) that achieve the targeted user satisfaction level. 
Chapter 2. Big Data-Driven and AI-based Framework to Enable Personalization in Wireless Networks

\subsubsection{Measure $Q_{o} S_{p}$ and get user satisfaction feedback}

After allocating resources to each user, the network records the $Q o S_{p}$ along with user feedback (i.e., satisfaction).

\subsubsection{Tuning}

The measured user satisfaction is used to validate the predicted satisfaction levels. If user satisfaction was predicted correctly, the instance is fed to the database. Otherwise, the error is fed to the predictive model to relearn (i.e., concept drift). Relearning is used to improve the predictive model performance and to update the model with user behavioral changes that could occur over time.

\subsubsection{Online learning}

Online learning is used to improve the predictive model proactively. Since network data become available in sequential order, batch learning techniques are not practical for real-time implementation. Instead, online learning techniques can dynamically adapt to new changes or patterns in user behavior and its relation to satisfaction.

In order to assess the practicality of the proposed framework, the steps that need to be performed during the communication session (i.e., online) should be assessed. As shown in Fig. 2.3, the production process involves relatively fast operations, such as data collection and performing predictions. However, utilizing optimization algorithms to solve data-driven resource allocation problems could potentially be a time-consuming process. Since optimizing resources in near realtime is essential for practical systems, meta-heuristics can provide sufficiently good solutions in a relatively short time. In contrast, the development and deployment stages may involve cumbersome, time-consuming processes, such as initial data collection, training, and validation, which require heavy computation. Nonetheless, since they are implemented offline, they should not affect the network proactivity.

\subsection{Illustrating the Benefits of Personalized Wire- less Networks}

In order to verify the benefits of personalized networks, the proposed framework is implemented as a proof-of-concept case study, which resembles a simplified, yet 
Chapter 2. Big Data-Driven and AI-based Framework to Enable Personalization in Wireless Networks

realistic network scenario. The prototype was implemented in python where the TensorFlow library was used to build the ML capabilities required for the framework. In this section, the implemented prototype is utilized to study the benefits of integrating personalization into wireless networks. For the purpose of comparison, two networks are implemented, a personalized and a non-personalized network. The personalized network utilizes the proposed framework to minimize the allocated resources (i.e., maximize $\Delta$ ) while maintaining user satisfaction levels higher than the targeted minimum satisfaction. On the other hand, since current cellular networks are designed to maximize $\mathrm{QoS}_{\mathrm{p}}$, the implemented non-personalized network optimizes the allocated resources to provide services with $\mathrm{Q}_{\mathrm{p}}$ as close as possible to $\mathrm{QoS}_{\mathrm{d}}$ (i.e., minimize $\Delta$ ) for all users.

\subsubsection{Experimental Setup}

\subsubsection{Cellular network environment}

Consider a cell within a cellular network that covers Ottawa, Canada. The cell has one eNB and it is connected to three active users moving within its coverage area. The area of the cell is divided into a $k * k$ grid. The cellular network environment is simulated using the parameters listed in Table 2.2. The cellular network operator collects data about the users and stores it in a database. The collected data are of two types, real-time user satisfaction levels as well as context values, such as time, location, and application. Measurements are recorded at each measuring instant. The period between two measuring instances is referred to as a Time Slot (TS). The operator collects data from the considered users with TS length of one

TABLE 2.2: Simulation parameters.

\begin{tabular}{|c|c|}
\hline \multicolumn{1}{|c|}{ PARAMETER NAME } & PARAMETER VALUE \\
\hline Number of eNBs & 1 \\
\hline Number of users & 3 \\
\hline Number of available RBs & 9 \\
\hline Number of subcarriers per RB & 12 \\
\hline Resource block bandwidth & $180 \mathrm{kHz}$ \\
\hline Carrier frequency & $2 \mathrm{GHz}$ \\
\hline Flat fading & Rayleigh \\
\hline Log normal shadowing & $8 \mathrm{~dB}$ standard deviation \\
\hline Distance attenuation & $\mathrm{L}=35.3+37.6 \times \log (\mathrm{d})$ \\
\hline UE thermal noise figure & $9 \mathrm{~dB}$ \\
\hline UE thermal noise density & $-174 \mathrm{dBm} / \mathrm{Hz}$ \\
\hline Grid size (k) & 100 \\
\hline Smin & 4 \\
\hline
\end{tabular}


second. Besides, the amount of resources used for each TS is recorded. Based on the type of service plan provided for the three users, the network operator targets a satisfaction level of $4\left(S_{\min }=4\right)$.

\subsubsection{Dataset description}

As shown in Fig. 2.3, user and network data are crucial requirements for personalized networks. Unfortunately, companies and institutions capable of collecting such data, particularly user data, do not publish them for privacy and confidentiality reasons. The way around this issue is to design and generate synthetic data that is flexible and has realistic characteristics. In Chapter 4, a synthetic dataset design is proposed to enable big data-driven wireless network personalization. The dataset is designed with four distinct user personas and is composed of context features, such as time, location, speed, and application along with their associated satisfaction labels. The dataset is utilized to build the prototype for the proposed personalized network and it can be found in a publicly available GitHub repository [39].

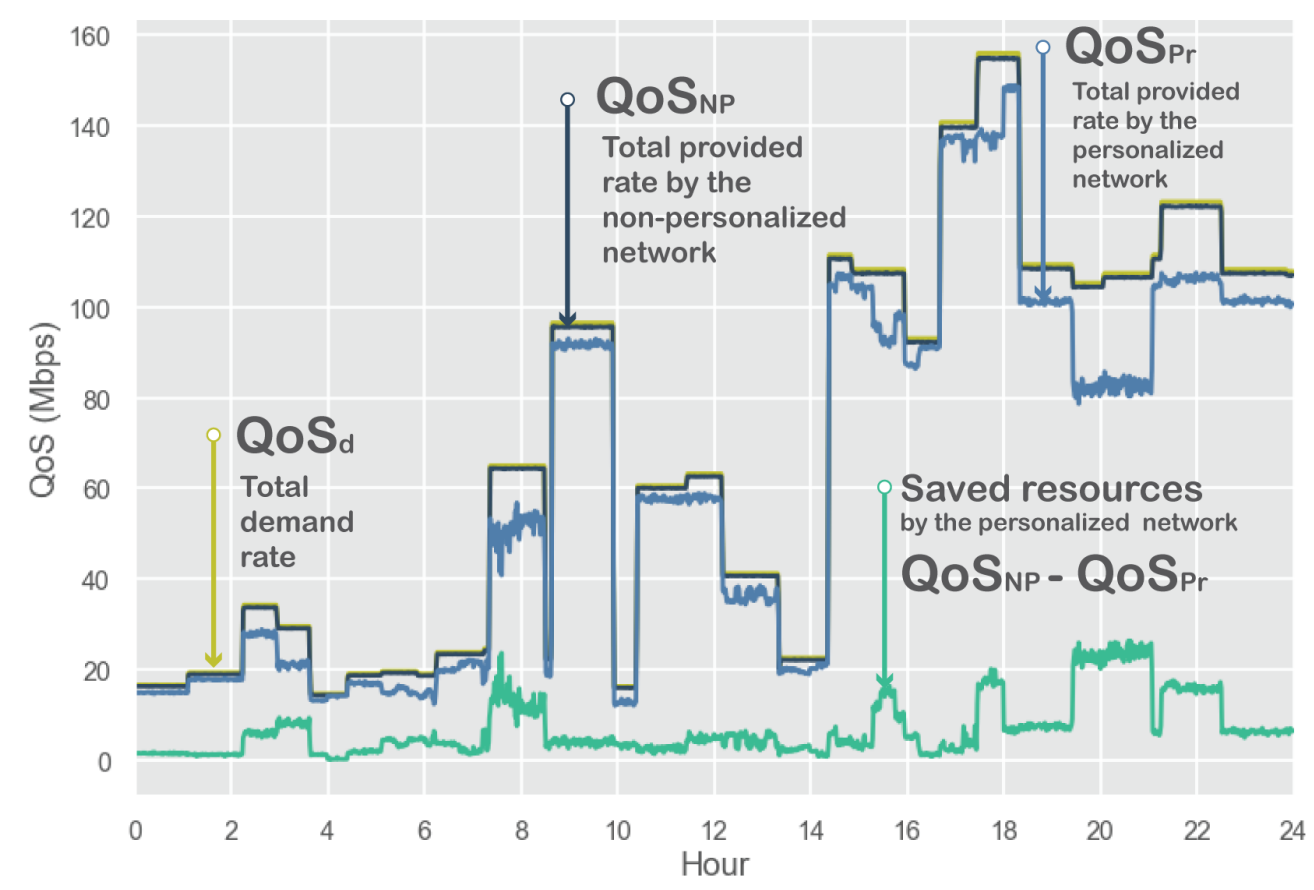

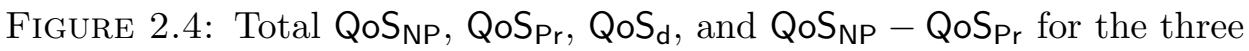
users vs. time in hours. 


\subsubsection{Experimental Results}

In this section, for the purpose of comparison, both personalized and non-personalized networks are simulated. The first premise of personalized networks is their ability to minimize the overall utilized resources at each instant (or frame). While resources in wireless networks are miscellaneous, in this chapter, resources are confined to Bandwidth, which is proportional to $\mathrm{Q}_{\mathrm{p}} \mathrm{p}$ in Mbps. The amount of saved resources is measured by calculating the difference between the $\mathrm{Q}_{\mathrm{o}} \mathrm{S}_{\mathrm{p}}$ provided by the non-personalized network $\left(Q_{0} S_{N P}\right)$ and $Q_{o} S_{p}$ provided by the personalized network $\left(Q_{0} S_{P r}\right)$ (i.e., QoS $\left.S_{N P}-Q_{0} S_{P r}\right)$. Fig. 2.4 depicts the total $Q \circ S_{N P}, Q_{0} S_{P r}$, $\mathrm{QoS}_{\mathrm{d}}$, and $\mathrm{QoS}_{\mathrm{NP}}-\mathrm{QoS}_{\mathrm{Pr}}$ for the three users vs. time in hours. As Fig. 2.4 shows, the total amount of saved resources $\left(Q_{0} S_{N P}-Q \circ S_{P r}\right)$ fluctuate with time. Essentially, the personalized network achieves the highest resource-saving when the non-personalized network attempts to maximize $\mathrm{QoS}_{\mathrm{p}}$ while the user has more tolerance to lower $\mathrm{Q}_{\mathrm{S}}$. In this particular scenario, as shown in Fig. 2.4, the amount of saved resources was always greater than zero indicating that the personalized network was able to provide service with $\mathrm{Q}_{\mathrm{O}} \mathrm{S}_{\mathrm{Pr}} \leq \mathrm{Q} \circ \mathrm{S}_{\mathrm{NP}}$; hence, it was able to save more resources (9703.8 Mbps over 24 hours) compared to the nonpersonalized network. However, depending on $S_{\text {min }}$, users' ZoTs, and the amount of resources provided to each user, the personalized network might suggest an

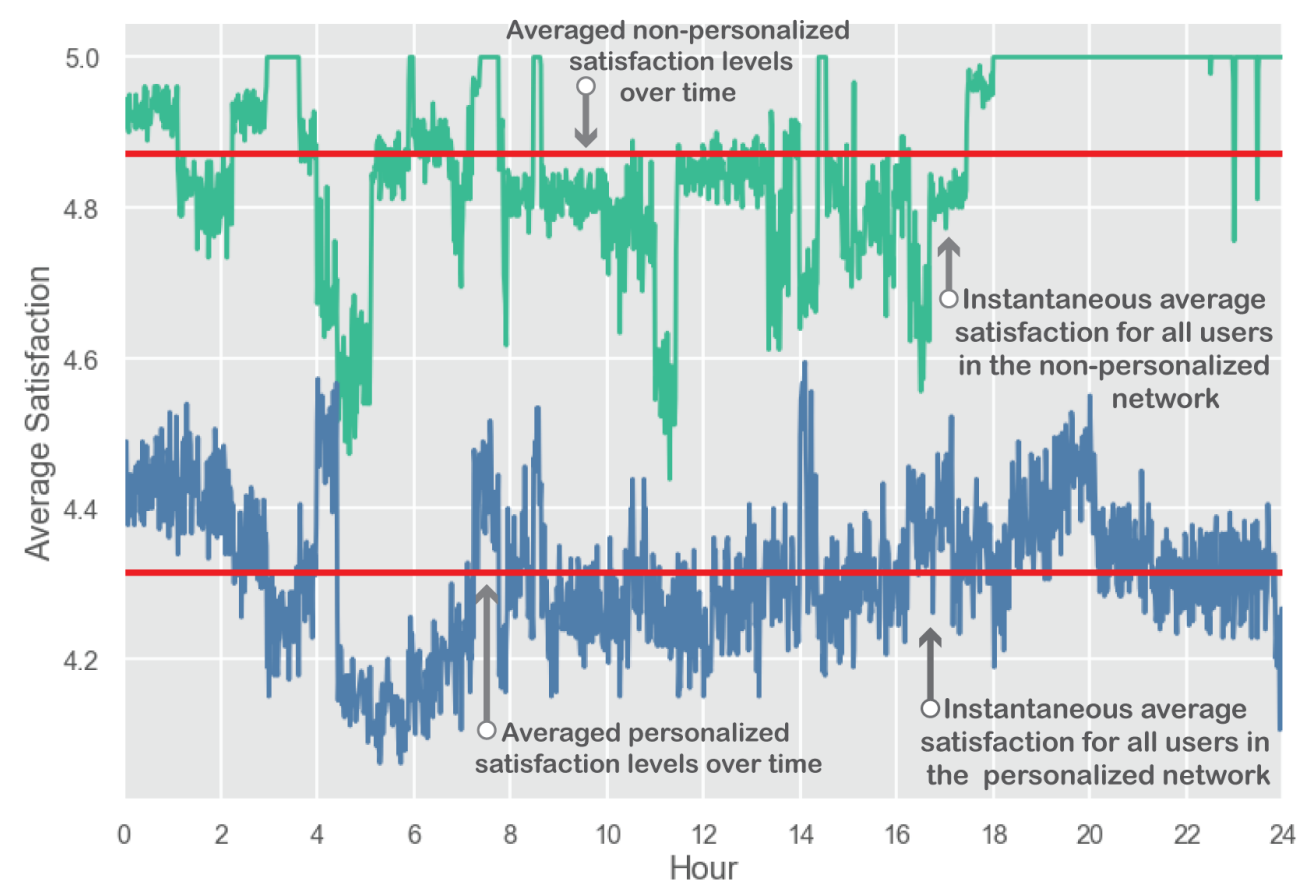

FIgURE 2.5: Average user satisfaction for the three users vs. time in hours for the personalized and non-personalized networks. 
increase in the provided resources to certain users (i.e., $\mathrm{Q}_{\mathrm{Pr}}>\mathrm{Q} \mathrm{O} \mathrm{S}_{\mathrm{NP}}$ ) to push their satisfaction levels above the targeted minimum. Usually, this increase in the provided resources for low tolerance users is offset by the reduced amount of provided resources for high tolerance users. In addition, the extra amount of resources suggested to low tolerance users is the optimized minimum required to achieve targeted satisfaction.

The second premise of personalized networks is their ability to maintain targeted satisfaction levels. To substantiate this claim, a comparison between the satisfaction levels of the personalized and non-personalized networks is provided. Fig. 2.5 depicts the average user satisfaction for the three users vs. time in hours for both networks. As shown in Fig. 2.5, although on average the non-personalized network achieved higher satisfaction levels (an average of 4.87), the personalized network was able to maintain user satisfaction above the targeted level of 4 (an average of 4.31) and save resources, simultaneously. 


\section{Chapter 3}

\section{User Satisfaction in Wireless Networks}

Wireless network personalization has two key enablers: measuring and predicting user satisfaction in real-time, and datasets that have both context and user satisfaction information. In order to enable user feedback tracking and measurement, it should be modeled and quantified. Therefore, this chapter introduces a novel user satisfaction model based on the zone of tolerance (ZoT) concept. The ZoT model is proposed for modeling the relationship between context, service performance, and user satisfaction. This chapter starts with a background on user satisfaction modeling and detection in wireless networks. In addition, the chapter discusses the ZoT model and the associated nonlinear mapper with illustrative examples. It is worth noting that the actual real-time user satisfaction feedback measurement using user sensor data is out of the scope of this thesis. 


\subsection{Background}

\subsubsection{Types of user satisfaction feedback}

The collection of user satisfaction feedback could be done in real time or offline in a number of ways that can be either intrusive or non-intrusive. Intrusive collection methods require users to actively interact with the system to record satisfaction information. On the other hand, non-intrusive user satisfaction collection methods employ ML and AI to predict personalized user satisfaction without the need to disturb users.

Examples of intrusive collection methods include surveys and feedback boxes. In wireless networks, the utilization of user feedback from intrusive methods is discussed in [40, 41]. The authors in [40] propose an approach called "user-inthe-loop" which utilizes real-time feedback to integrate spatial demand control to wireless networks where users are motivated to move to less congested areas. The authors in [41] propose a data-guided resource allocation approach where offline feedback data (e.g., network measurements and user complaints) is employed to improve the average user experience.

Generally, the intrusive feedback collection methods do not represent all users because the majority of users wouldn't complain, they just change the provider. Furthermore, while users' needs and expectations change rapidly in wireless networks, non-intrusive feedback collection methods enable more frequent feedback data collection which, consequently, increases the accuracy and relevance of network decisions. Therefore, non-intrusive feedback methods are considered more practical compared to intrusive feedback. Nonetheless, due to the lack of data and the immaturity of the technology required to acquire and utilize non-intrusive user satisfaction feedback in wireless networks, it is not a common discussion topic in the literature and is limited to but a few applications [42]. Nevertheless, nonintrusive user feedback has been proposed to make relevant automotive decisions in many applications, such as cloud gaming, healthcare, and human-computer interaction [43-45]. In this thesis, the proposed framework will enable non-intrusive user satisfaction feedback collection in wireless networks. There are several methods that can be used to collect and manage context data and non-intrusive satisfaction information collection, one of which is through an application installed on users' devices (developed and maintained by the service provider). 


\subsection{User Zone of Tolerance Model}

Due to the intense competition in the telecommunication industry, user satisfaction is crucial to sustaining a profitable business for any service provider. Over the long run, service providers that can maintain user satisfaction will obtain and retain more subscribers and increase business growth. "Satisfied customers offer businesses a promise of enhanced revenues and reduced operating costs" [46]. In order to understand customer/user satisfaction, business and marketing studies proposed the model of service quality. This model utilizes the Zone of Tolerance (ZoT) notion, which is defined as "the range of service performance a customer would consider satisfactory." The model of service quality depicts the ZoT as the service range bounded by desired and adequate levels of service. The desired level of service is defined as "the level of service representing a blend of what customers believe can be and should be provided," whereas the adequate level of service is defined as "the minimum level of service customers are willing to accept."

Drawing on concepts of service quality from business and marketing studies, our model of user satisfaction $(S)$ in wireless networks is shown in Fig. 3.1. The proposed model suggests dividing user satisfaction into levels where each level is associated with a certain range of QoS. In Fig. 3.1, satisfaction is divided into four levels: A, B, C, and D. The division and number of satisfaction levels could vary depending on service providers' preferences. Before proceeding to the detailed discussion of the user satisfaction model, it is worth mentioning that QoS can

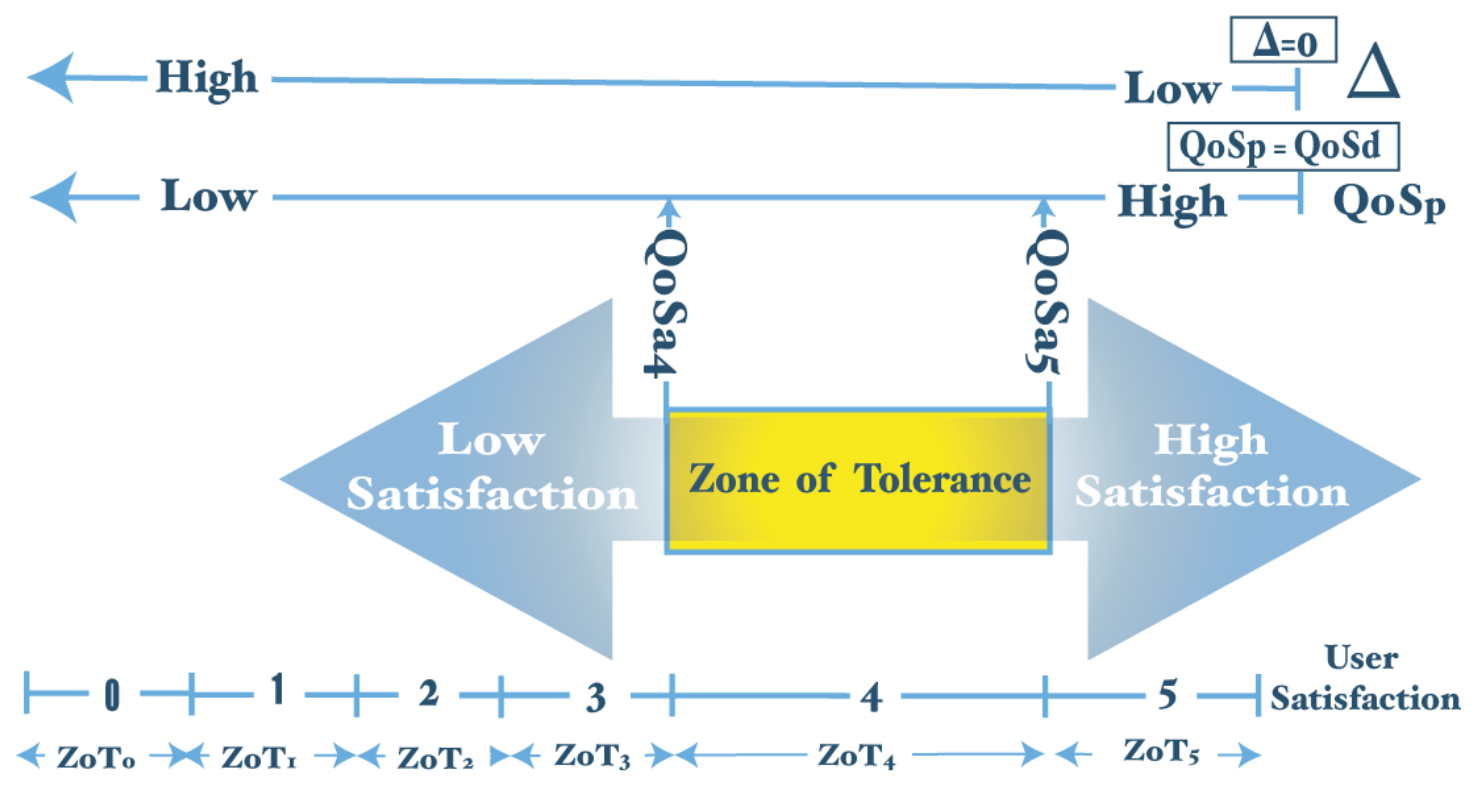

Figure 3.1: User Zone of Tolerance (ZoT) model. 
be a vector with several elements, such as rate, reliability, latency, and jitter. Nonetheless, for simplicity, QoS is assumed to be solely defined by rate. Our proposed user satisfaction model encompasses the following five main notions:

- QoS : the demanded QoS by the user, which represents the maximum QoS associated with the requested service.

- $Q S_{p}$ : the provided QoS by the network.

- $Q_{o} S_{a_{i}}$ : the adequate (minimum) QoS required to achieve a satisfaction level of $i$.

- $Z o T_{i}$ : the QoS range that satisfies the user with a satisfaction level of $i$. For $i<5, Z o T_{i}$ ranges between $Q o S_{a_{(i+1)}}$ and $Q o S_{a_{i}}$, whereas for $i=5, Z o T_{5}$ ranges between $Q_{o} S_{d}$ and $Q_{o} S_{a_{5}}$.

- $\Delta$ : the difference between the QoS demanded by the user and the QoS provided by the network $\left(Q_{o} S_{d}-Q_{o} S_{p}\right)$.

As shown in Fig. 3.1, as $Q o S_{p}$ decreases, $\Delta$ increases and, consequently, satisfaction decreases. To keep user satisfaction at a certain level, $Q_{o} S_{p}$ should be within the ZoT associated with the targeted satisfaction level. It is important to note that $Q o S_{a_{i}}$ is what changes from one user to another, which consequently changes the width of the $Z o T_{i}$. Moreover, demand is assumed to be dependent on the application and service type; hence, it is constant for all users requesting service of the same application.

\subsection{Zone of Tolerance in the Context of Person- alized Networks}

Understanding and characterizing ZoT will open the door to micro-managing wireless networks, which will allow operators to personalize their services and design new business models to generate new revenue streams while maintaining the targeted user satisfaction levels. Fig. 3.2 presents a simple example to illustrate how ZoT, $\Delta$, and user satisfaction are related. Assume that a service provider is trying to optimize the network such that a certain user in the network has a satisfaction level of B. Furthermore, assume the service provider is utilizing a big data-driven AI personalized network to predict $Z o T_{B}$ at different time slots $\left(T_{1}\right.$ to $\left.T_{6}\right)$. Based on the predicted $Z o T_{B}$, the personalized network optimizes $\Delta$ during each time 


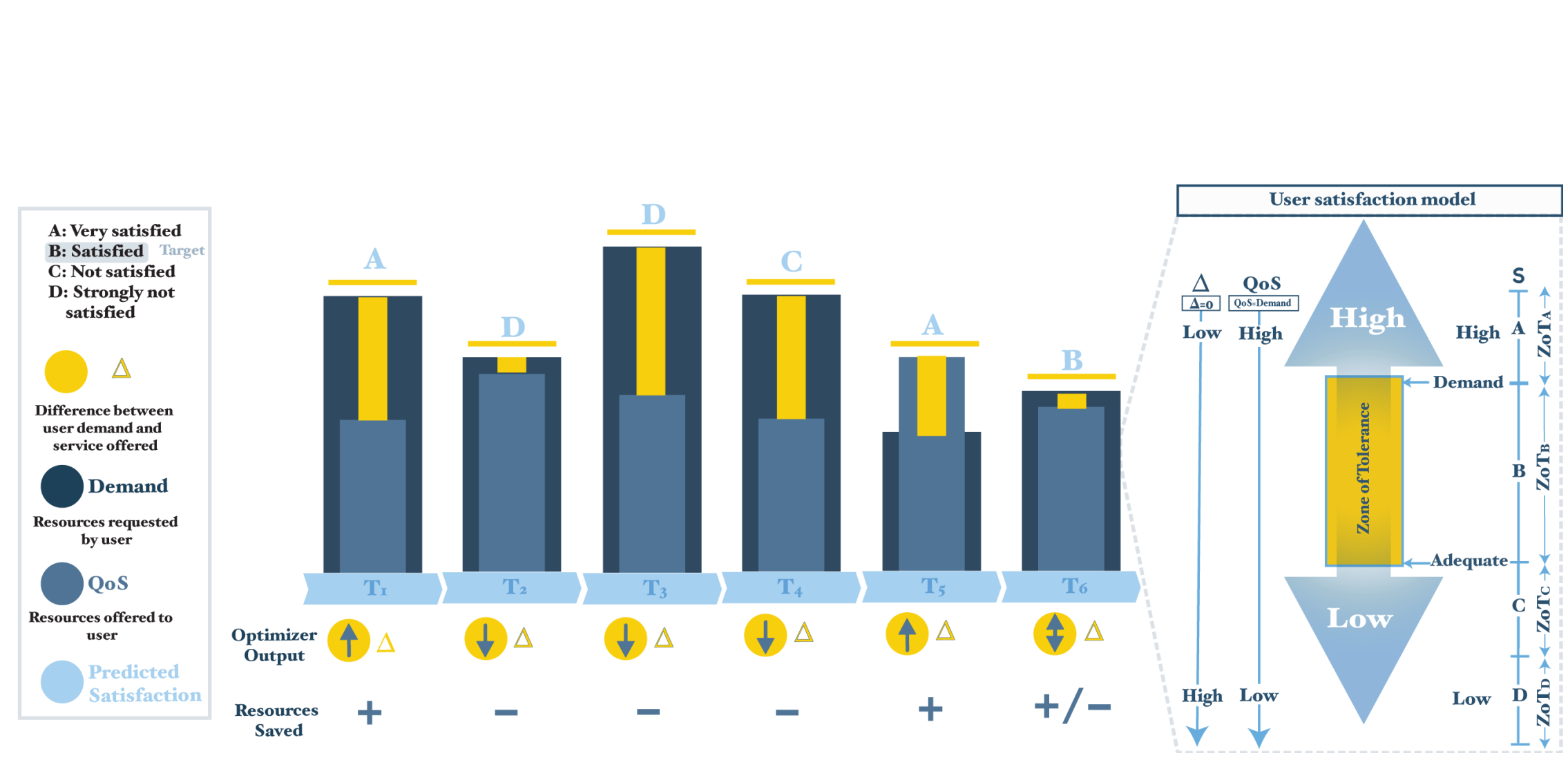

FIGURE 3.2: User satisfaction model and a visualization of an example illustrating the relationship between the zone of tolerance, $\Delta$, user satisfaction, and personalized network decisions. 

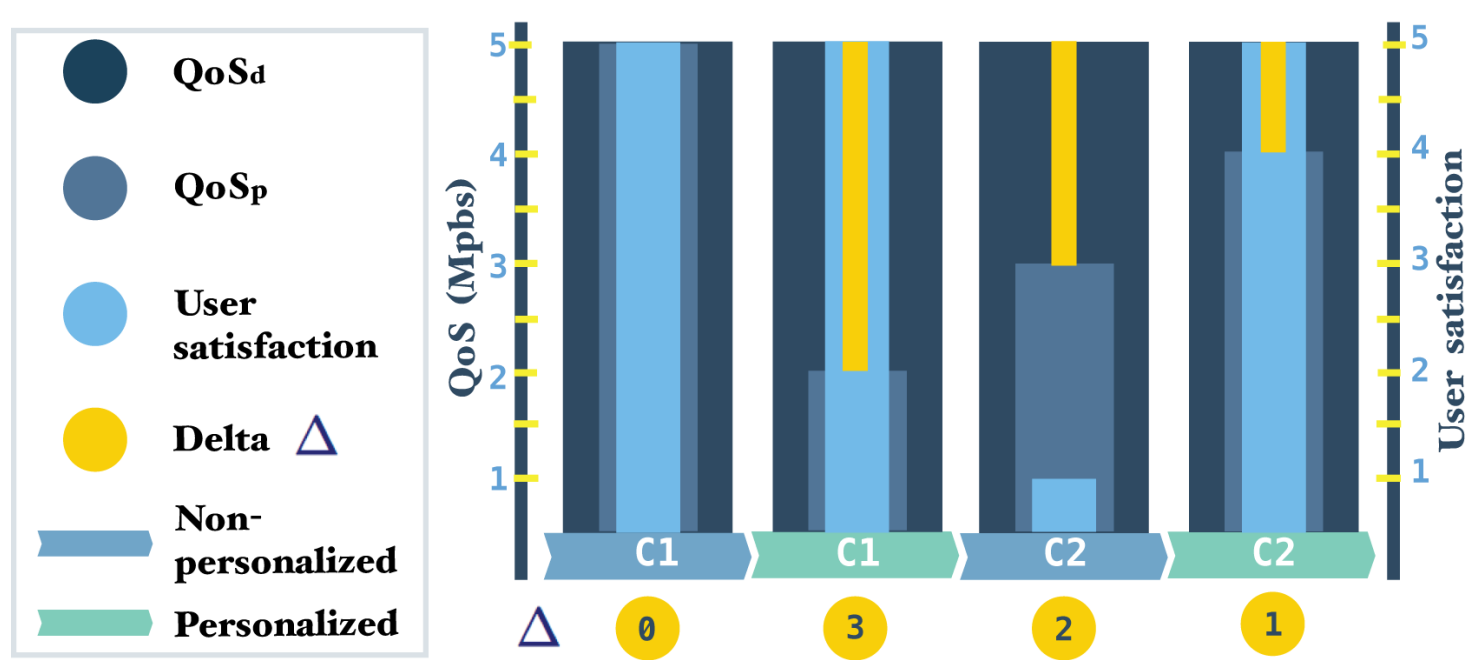

FIGURE 3.3: $Q o S_{d}, Q o S_{p}, \Delta$, and the corresponding user satisfaction for contexts $C_{1}$ and $C_{2}$ associated with both personalized and non-personalized networks.

slot. As shown in Fig. 3.2, at $T_{1}$, based on the provided $\Delta$, the predicted user satisfaction level is A. In order to reduce satisfaction and consequently save resources, the optimizer, which is part of the personalized network, suggests increasing $\Delta$ (1) to save resources $(+)$. At $T_{2}$, since $Z o T_{B}$ is continuously changing over time, although the provided $\Delta$ is lower than $\Delta$ at $T_{1}$, user satisfaction drops to level D. This indicates that this user has a very tight $Z o T_{B}$ at $T_{2}$, and consequently the optimizer suggests decreasing $\Delta(\mathbb{})$ further by allocating more resources (-). At $T_{6}$, the predicted user satisfaction for the provided $\Delta$ is level $\mathrm{B}$, which is the targeted user satisfaction. Therefore, the optimizer suggests keeping $\Delta$ as is $\mathbb{1}$ $(+\backslash-)$.

\subsection{How Can Personalization Potentially Save Resources and Increase User Satisfaction?}

In this section, using the proposed ZoT model, a few exemplary instances shall be considered in order to illustrate how personalization can potentially save resources and improve satisfaction at the minimum cost. Fig. 3.3 illustrates two different contexts, $C_{1}$ and $C_{2}$. The value of $\Delta$ is illustrated by the length of the yellow bar and is depicted, for each context, in the yellow circles. The targeted user satisfaction for the considered user is assumed to be 5 and the $Q o S_{d}$ is $5 \mathrm{Mbps}$. To begin with, the data associated with $C_{1}$ is analyzed. As for the non-personalized network, the allocated $Q_{o} S_{p}$ was $5 \mathrm{Mbps}$, and consequently, $\Delta$ will sum up to 
zero Mbps. On the other hand, the personalized network allocated only 2 Mbps, causing $\Delta$ to rise to $3 \mathrm{Mbps}$. The personalized network was able to predict that this user, during $C_{1}$, would have a relatively large $Z o T_{5}$ and, hence reduce the amount of resources allocated by $3 \mathrm{Mbps}$ without sacrificing user satisfaction. Accordingly, the following can be concluded: personalization can potentially save resources during contexts with larger ZoT without sacrificing user satisfaction.

On the contrary, during $C_{2}$, the non-personalized network allocated $3 \mathrm{Mbps}$, whereas the personalized network allocated 4 Mbps to reduce $\Delta$ from 2 to 1 Mbps. As a result, user satisfaction climbed from 1 for the non-personalized network to 5 for the personalized network. Personalization enabled the network to predict that this user, during $C_{2}$, would have a smaller $Z o T_{5}$, and hence the minimum required $Q o S_{p}$ is $4 \mathrm{Mbps}$. Accordingly, the following can be concluded: personalization can potentially increase user satisfaction to the desired level during contexts with tighter ZoT and using a minimum amount of resources.

\subsection{User Satisfaction Generation Model}

User satisfaction is assumed to be correlated with context and $\Delta$ (see Fig. 3.1). In order to generate satisfaction values $(\mathcal{S})$ based on the proposed ZoT model, the following non-linear mathematical formulation is proposed:

$$
\begin{aligned}
& \mathcal{S}\left(\psi, \Delta, \Delta_{\text {max }_{0}}, \lambda\right) \approx \\
& \begin{cases}\alpha_{h} \cdot \beta_{h}^{\left(\frac{\Delta . \zeta . \lambda}{\Delta_{\text {max }}}\right)}+6 & , \text { for } \psi=h, \Delta \leqslant \Delta_{\text {max }_{0} .} \\
\alpha_{l} \cdot \beta_{l}^{\left(\frac{\Delta . \zeta . \lambda}{\Delta \text { max }_{0}}\right)} & , \text { for } \psi=l, \Delta \leqslant \Delta_{\text {max }_{0} .} \\
5 & , \text { for } \mathcal{S}>5 . \\
0 & , \text { for } \Delta \geqslant \Delta_{\text {max }_{0}} \| \mathcal{S}<0\end{cases}
\end{aligned}
$$

where $\alpha_{h}=-1, \alpha_{l}=5, \beta_{h}=1.001, \beta_{l}=0.999$, and $\zeta=1750$. $h$ refers to high tolerance behavior, whereas $l$ refers to low tolerance behavior. The values of the aforementioned variables are tuned such that the proposed nonlinear mapper in (3.1) reflects the proposed ZoT satisfaction model. Also, the nonlinear model in (3.1) is chosen to echo the jumps in satisfaction with the varying $\Delta$. Since the minimum rate a user could receive is zero, $\Delta_{\max }$ is equal to user demand (i.e., $\left.\Delta_{\max }=Q_{o} S_{d}-0\right)$. User satisfaction behavior at a specific context is modeled using two variables, $\lambda$ and $\psi$. As $\lambda$ decrease, $Z o T$ and satisfaction increase, and vice versa. Also, a satisfaction mapper with $\psi=l$ indicates tighter $Z o T$ s for 


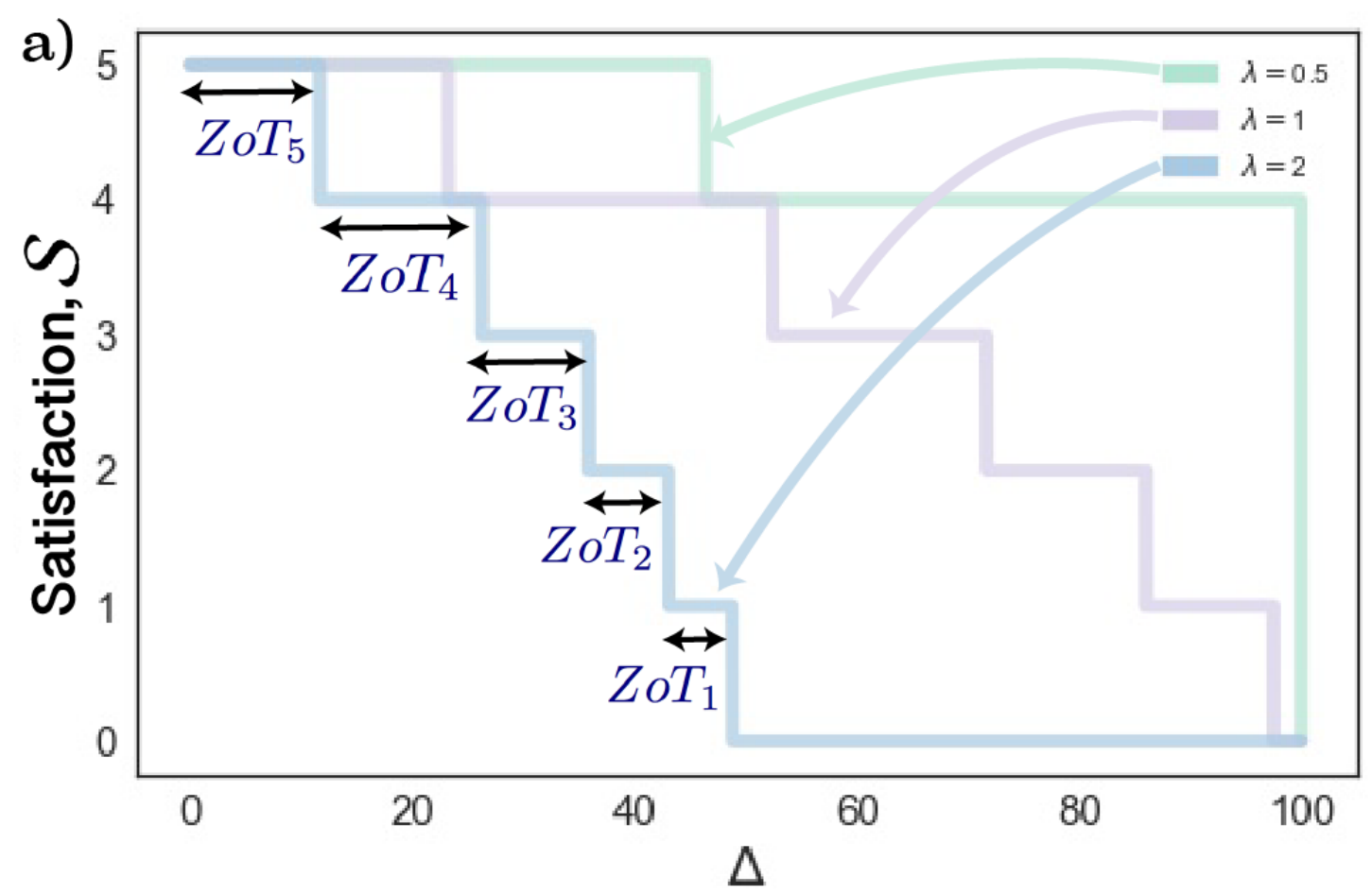

FiguRE 3.4: Satisfaction mapper for $\psi=h$ and $\lambda=0.5,1,2$

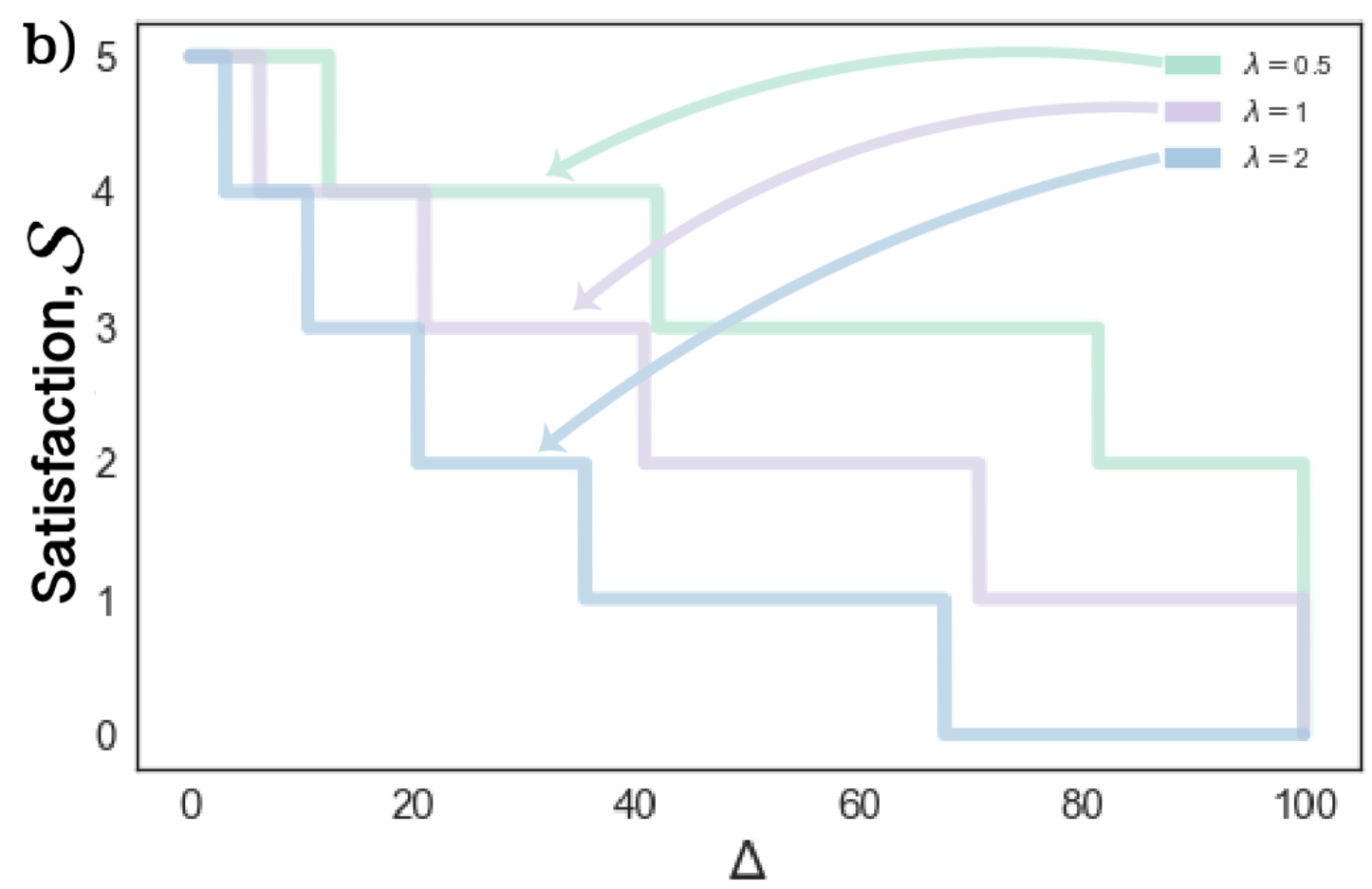

Figure 3.5: Satisfaction mapper for $\psi=l$ and $\lambda=0.5,1,2$. 
higher satisfaction values compared to lower ones. Similarly, $\psi=h$ indicates a more relaxed $Z o T$ s around higher satisfaction values compared to lower ones. Notably, at a certain context, $\lambda$ and $\psi$ has fixed values and user satisfaction model is a function of $\Delta$. It is worth menetioning that personlized netwroks save more resources for users with high $\lambda_{\mathrm{s}}$ (higher tolerance) compared to users with low $\lambda \mathrm{s}$ (lower tolerance).

Fig. 3.4 and 3.5 visualize the $\mathcal{S}$ mapper in (3.1) for $\psi=h$ and $\psi=l$, respectively, in conjunction with $\Delta_{\max }=100$ and different values of $\lambda$. From Fig. 3.4 and 3.5, it can be deduced that for a certain context (i.e., fixed $\psi, \Delta_{\max }$, and $\lambda$ ), $\mathcal{S}$ is solely a function of $\Delta$. Moreover, for the same $\lambda$ value, $\mathcal{S}$ with $\psi=h$ (see Fig. 3.4) has greater ZoTs compared to $\mathcal{S}$ with $\psi=l$ (see Fig. 3.5). Finally, another significant aspect of the proposed mapper in (3.1) is that $Z o T$ s increase with the decrease of $\lambda$ for all satisfaction values. In this thesis, each context is associated with a certain combination of $\lambda$ and $\psi$. 


\section{Chapter 4}

\section{A Synthetic User Behavior Dataset Design for Data-driven AI-based Personalized Wireless Networks}

Data-driven personalization will empower wireless networks to further optimize resources while maintaining user expectations of networks. In order to design, test, and validate research ideas related to wireless network personalization, acquiring data is essential. However, datasets that comprise user behavior and corresponding user satisfaction information are generally not published due to privacy and confidentiality concerns. To account for this, in this chapter, a synthetic dataset design methodology is proposed to generate labeled user behavior data with ground truth satisfaction values that mimic the real characteristics of real datasets. Besides, sample user satisfaction prediction experiments are conducted using several ML algorithms. 
As mentioned earlier, user satisfaction depends on both context data and $\Delta$ values. Despite the possibility of measuring context variables, satisfaction, and $\Delta$ values, a dataset that has the three elements does not exist in published literature. Therefore, in this chapter, a process is designed to synthesize a dataset based on the proposed user satisfaction model. All datasets generated in this chapter are generated for one year. Theoretically, there is a relatively large number of context variables of which each has a different effect on the satisfaction behavior of users. However, for the sake of this discussion, the following context variables are considered: time, day, location, speed, activity, service request arrival, application choice, application service choice, request rate demand, given rate, and $\Delta$. In this chapter, the details of generating datasets for users which comprise the aforementioned context variables along with the corresponding satisfaction values are discussed. The datasets generated in this chapter are publicly available in a GitHub repository [39].

One of the main design requirements of synthetic datasets is that they need to be similar to real-world data. However, designing a dataset with realistic characteristics is not a straightforward task. Real-world data are complicated and data attributes have internal patterns, errors, and dependencies most of which are nonlinear and difficult to model. In addition, the generated dataset should span a wide range of data space. In order to integrate the aforementioned design aspects into the proposed dataset, four strategies are proposed. The first strategy is to generate context variables with the proper data correlation. To achieve this, a methodology that is called the tree data generator is proposed. The second strategy is to introduce the notion of user persona model in order to create data for different users with different behavioral patterns and lifestyles. The third strategy is to integrate real-world sensor measurements in order to add real noise and errors to the generated data. Finally, the fourth strategy is to artificially integrate noise into user satisfaction values. Since real user satisfaction data does not exist yet, analyzing and modeling the actual behaviour of satisfaction noise can not be done yet. Therefore, and for the sake of simplicity, Gaussian noise is considered.

This chapter starts with a discussion on the benefits of utilizing synthetic datasets to study wireless network personalization. Afterward, in Section 4.2, the proposed generator utilized to create features with realistic correlations is discussed. Then, in Section 4.3, the generated data features and their values are described. Finally, Section 4.4 discusses the remaining four strategies used to integrate realistic characteristics to the generated datasets. 


\subsection{Pros and Cons of Utilizing Synthetic Datasets to Study Personalized Networks}

There are several advantages of employing synthetic data as opposed to real-world data. First and foremost, synthetic data removes privacy and confidentiality concerns. A lack of published user behavior data labeled with ground truth user satisfaction information is holding back innovation into new approaches for personalizing wireless networks. Companies and institutions capable of collecting such data do not publish them for privacy and confidentiality reasons. The way around this issue is to design and generate synthetic data that is flexible and has realistic characteristics. In addition to that, synthetic data can be generated in large volumes and with complex well-understood characteristics. Another fundamental advantage of synthetic datasets is the ability to redesign and change data structures as needed by varying certain input parameters. So instead of relying solely on real data over which we have little or no control, synthetic data provide us with great flexibility. Along with design flexibility, synthetic data generation methods enable the generated datasets to cover most of the data space needed to generate meaningful results, and hence, provide us with deeper insight and stronger conclusions. Moreover, the quality of the generated data can be controlled using various design techniques [47].

For all its benefits, the advantages of synthetic data come at a price. Real data are usually dirty and contain various types of errors; hence, it can be foreseen that the creation of data with characteristics similar to real-world data is not straightforward. Synthetic data also needs to reflect errors, distributions, and patterns that exist in real data. Finally, an additional validation step might be necessary to ensure that the conclusions drawn from synthetic data extend to real-world applications.

\subsection{Datasets Generation Model : Tree Data Gen- erator}

The Tree data Generator (TG) is a structure that specifies the relationships and correlation between context variables in user behavior generated datasets. This structure defines the rules, patterns, and dependencies that the generated data need to follow. In Fig. 4.1, a sample TG structure is illustrated. Each TG is a collection of several nodes, where each node has a value and could have a child 
node. Nodes that do not have child nodes are called leaf nodes, whereas nodes with child nodes are called parent nodes. The first node in TGs is called the key attribute node (see Fig. 4.1). Since context information datasets are collected over time, the key attribute node will always be time.

One of the main advantages of using TG to generate context data is its ability to exclude impossible or unwanted variable combinations. In other words, all paths in a TG will produce relevant data records. For instance, TG will eliminate speed values of zero when the recorded activity is running. TG nodes are of two types: rule-based nodes and Hidden Markov Model-based (HMM) nodes [48]. The values of rule-based nodes are generated based on rules specified by TG inputs. For example, the TG in Fig. 4.1 has a rule-based node called WeekDays (WD). WD node has two possible values: weekday and weekend. In order to determine whether a day is a weekday (working day) or a weekend day, a rule is integrated into this node. HMM nodes, by contrast, compute the state sequence conditional probabilities and perform a weighted random choice based on the computed probabilities.

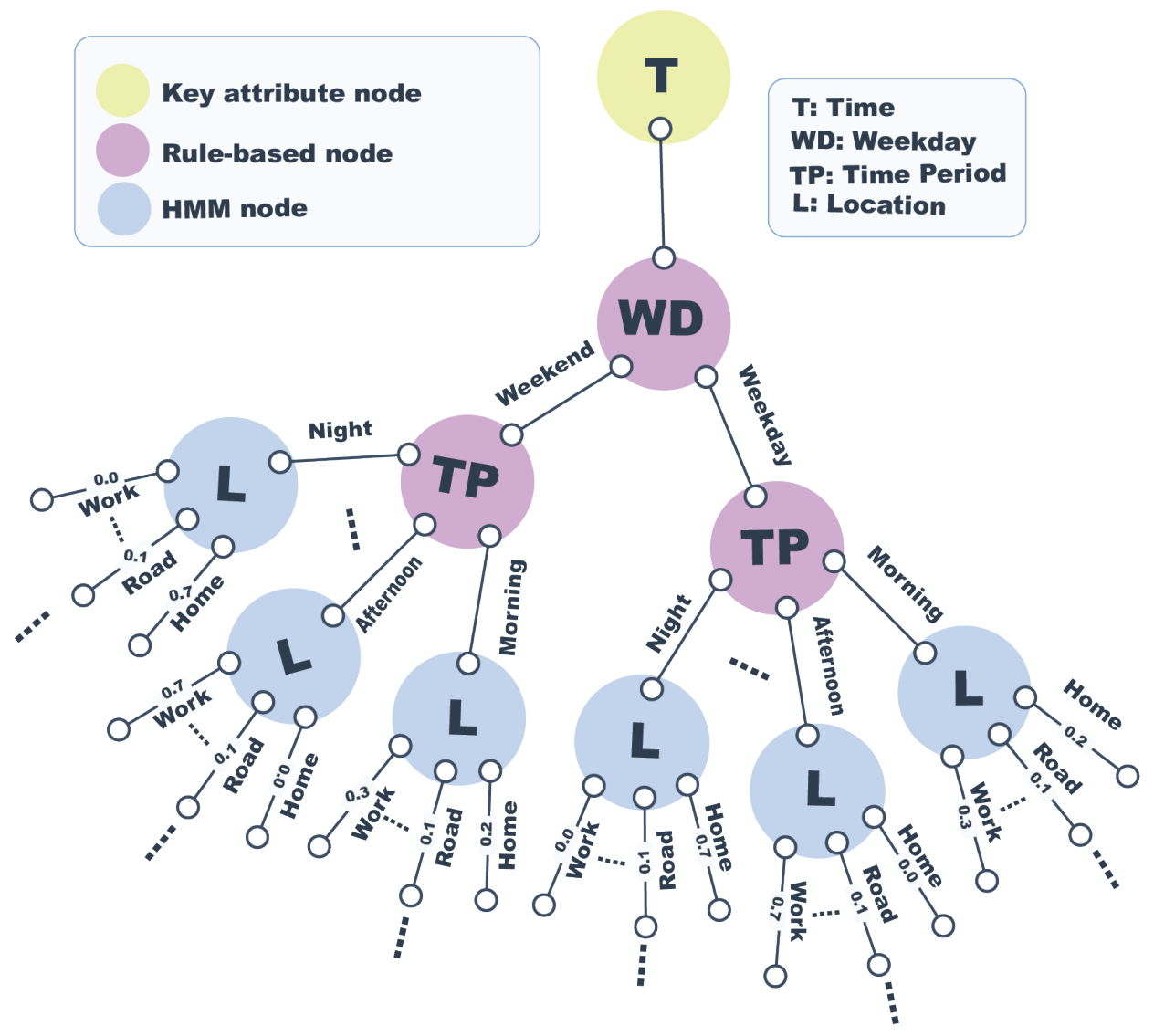

FIgURE 4.1: TG for a working professional persona. 


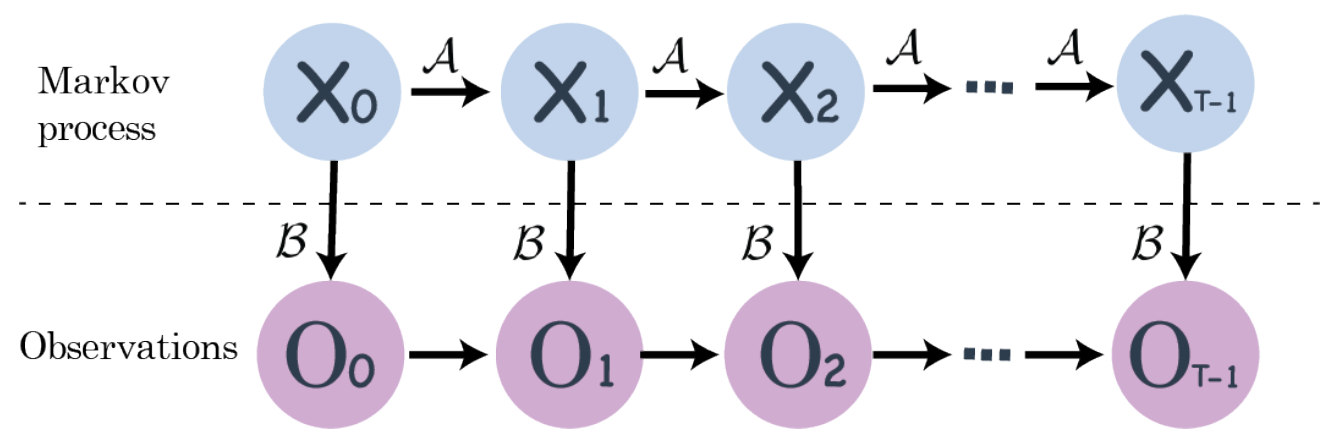

FiguRE 4.2: Graphical model representation of hidden Markov model.

Fig. 4.2 illustrates the decision process of HMM nodes, where $\mathcal{X}_{i}$ represents a hidden state sequence, $\mathcal{A}$ is the state transition probabilities, and $\mathcal{B}$ is the observation probability matrix. The matrix $\mathcal{A}=\left\{a_{i j}\right\}$ is $\mathbf{N} \times \mathbf{N}$ with

$$
a_{i j}=\mathbf{P}\left(\text { state } q_{j} \text { at } t+1 \mid \text { state } q_{i} \text { at } t\right)
$$

where $t$ is the current time, $\mathbf{N}$ is the number of states in the model, and $\mathcal{Q}=$ $q_{0}, q_{1}, \ldots, q_{N-1}$ are the distinct states of the Markov process. States of a TG's HMM node are the set of all possible values of the variable associated with the node. The matrix $\mathcal{B}=\left\{b_{i j}\right\}$ is $\mathbf{N} \times \mathbf{M}$ with

$$
b_{i j}=\mathbf{P}\left(\text { observation } k \text { at } t \mid \text { state } q_{i} \text { at } t\right),
$$

where an observation $\left(\mathcal{O}_{t}\right)$ of an HMM node at time $t$ is the sequence of values recorded at the predecessor parent nodes and $\mathbf{M}$ is the number of observed symbols.

\subsection{Features Description}

It is common these days for users to carry or wear electronic devices (e.g., smartphones, smartwatches) that have built-in sensors, such as microphones, dual cameras, accelerometers, gyroscopes, and more. Users can, therefore, be considered a rich source of context data. An example of a user context information dataset can be found in $[49,50]$. The availability of such context data presents new opportunities to develop new technologies that enable user-aware decisions in many applications. Although some context information datasets exist, large scale context information datasets, as well as datasets that have both context data and the corresponding satisfaction values, are not publicly available. 


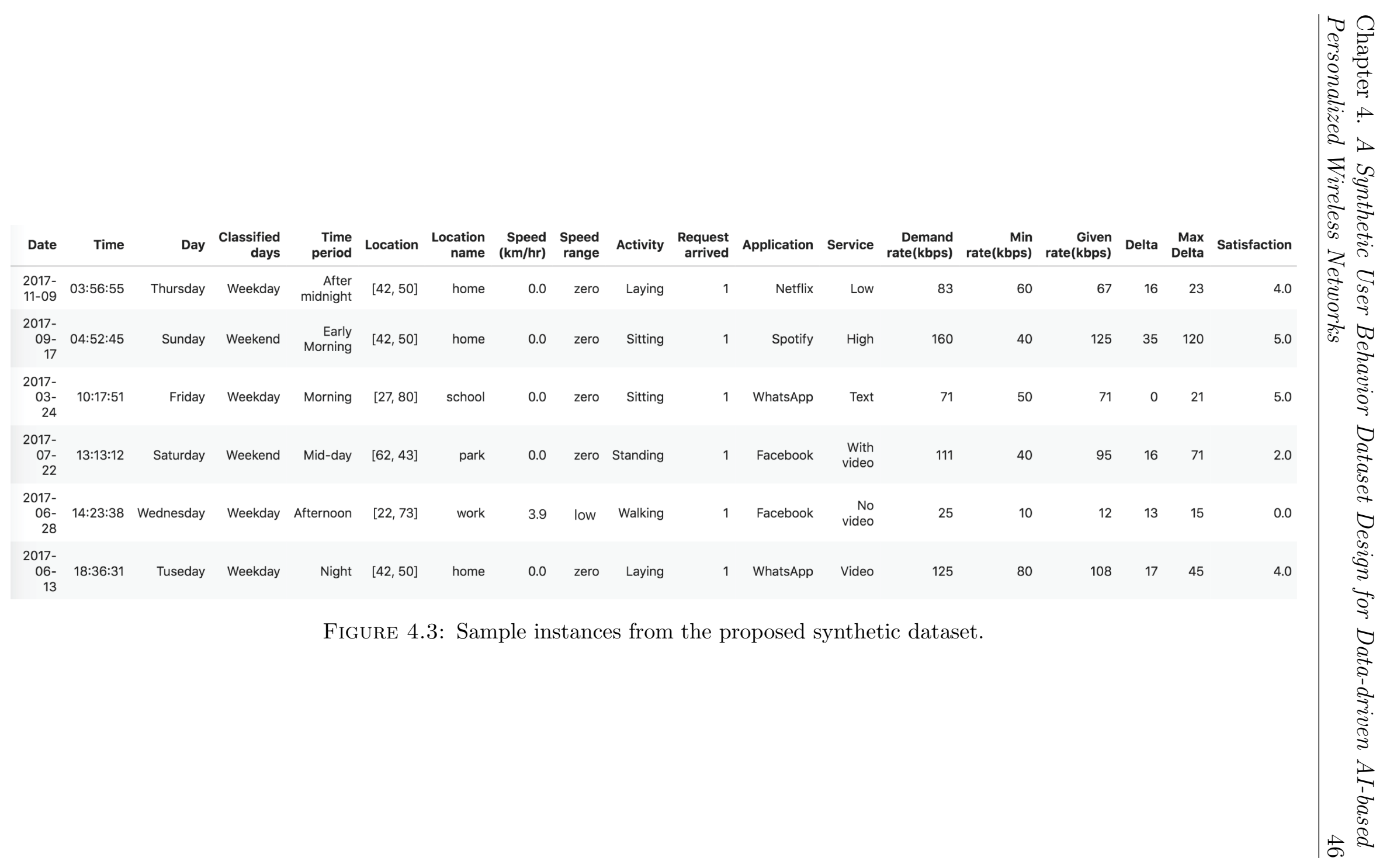


The proposed dataset structure consists of user context data and the corresponding satisfaction values. Context data are composed of a set of context variables, such as time and location. In this section, the measurements recorded are generated for one user over one year. The model used to design each context variable is also presented. In this dataset, a context variable could be a measured or engineered feature. Measured features, such as time, day, location, and speed are directly obtained from sensors. By contrast, engineered features, such as activity, are created on the basis of the measured features. Measured and engineered features are recorded at each measuring instant. Sample instances from the generated dataset are illustrated in Fig. 4.3. The period between two measuring instants is referred to as a Time Slot (TS). For the purpose of this dataset, the TS length is assumed to be 1 second. In order to model a realistic synthesis of user context with the proper correlation, The proposed TG generator is utilized to integrate relations between features (i.e., context variables). Fig. 4.4 illustrates these relations between four examples of features. Nodes represent features and links represent relations between features.

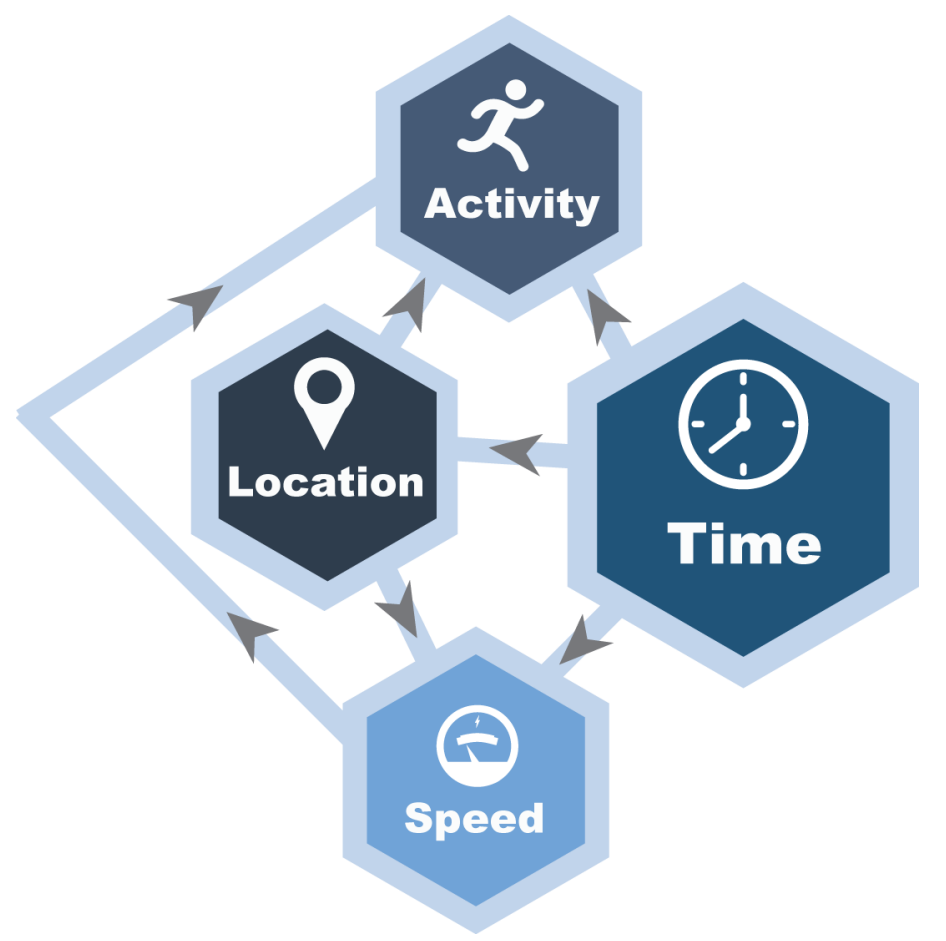

FiguRE 4.4: The relationships between the features: time, location, speed, and activity. 

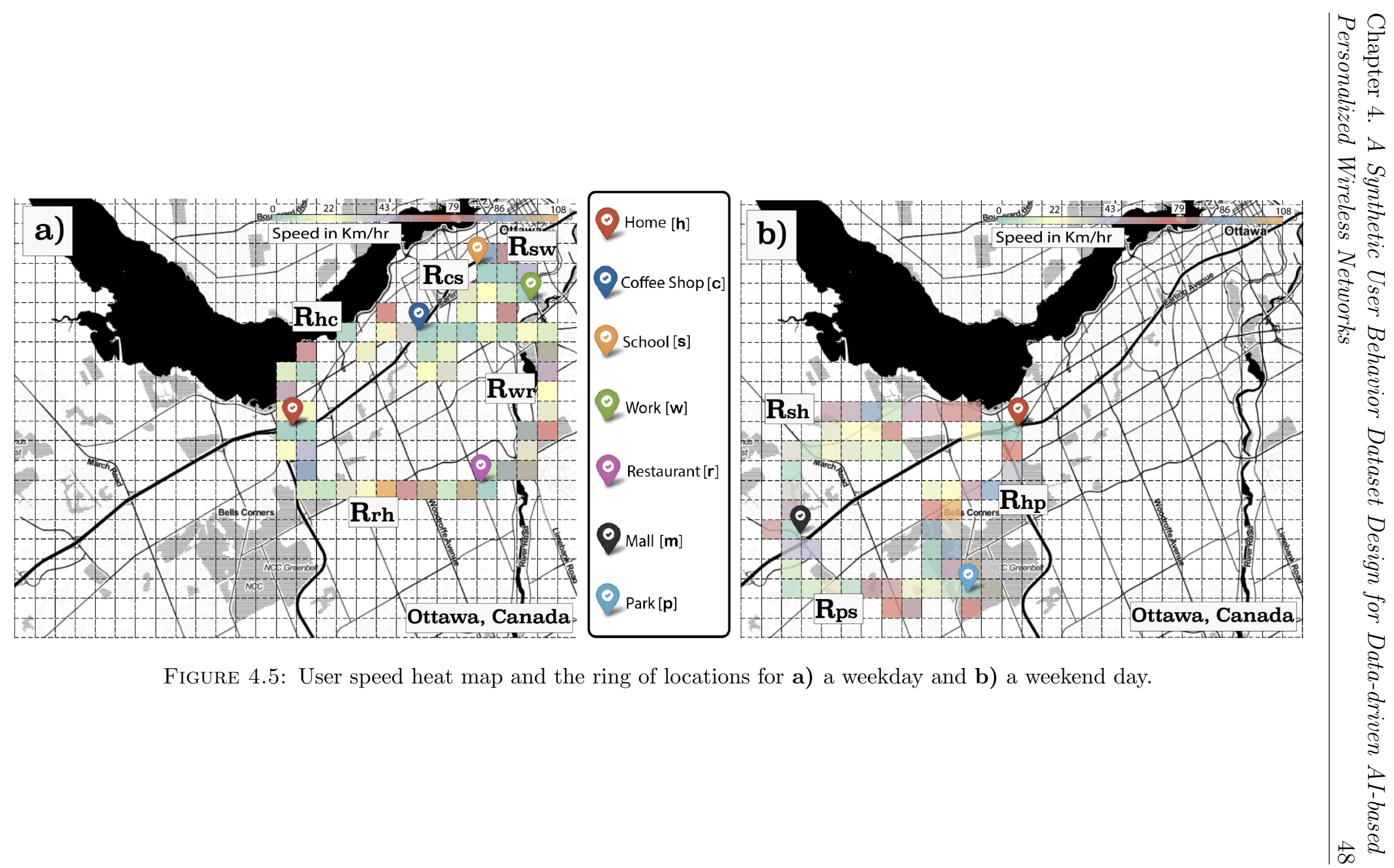


\subsubsection{Date, time, and day}

Date, Time of the day, and Day of the week are among the measured features added to the dataset. In addition, days of the week are classified into weekdays and weekends to create the Classified days engineered feature. Moreover, the time of the day is classified into 7 periods to create the Time period engineered feature. Time periods are assigned as follows: Early Morning: 04:00AM-05:59AM, Morning: 06:00AM-10:59AM, Mid-day: 11:00AM-13:59PM, Afternoon:14:00PM17:59PM, Night: 18:00PM-23:59PM, After midnight: 00:00AM-03:59AM.

\subsubsection{User location}

The user considered for this dataset is assumed to live in Ottawa, Canada. Instead of recording the user's GPS location, the user Location feature is engineered, which is created by dividing the Ottawa area into a $100 * 100$ grid with a distinct ID for each square. The area covered by each square is $91 \times 10^{-5} \mathrm{Km}^{2}$ and the distinct location ID is recorded to the dataset under the Location feature. Usually, most people follow location patterns in their daily life. Although these patterns do not occur with $100 \%$ probability, for the purpose of this dataset, it is assumed that the user has a certain and distinct location pattern for weekdays and weekends. In this work, the set of roads and destinations that constitute a location pattern is called the ring of locations. Roads and destinations are added to the dataset under the Location name feature. Roads are named $R_{i j}$, where $i$ is the initial of the starting location and $j$ is the initial of the destination. For example, $R_{h p}$ refers to the road from home to the park. Fig. 4.5.a and Fig. 4.5.b depict the weekday and weekend rings of locations for the considered user, respectively. The dependency of location on time is modeled using Markov chain probability with transition probabilities dependent on the next destination, time period, and current location. In Fig. 4.6, in order to illustrate the correlation between the time period and location features, the percentage of time the user spent at each location is plotted over each time period on a weekday. From Fig. 4.6, it can be deduced that the user spends most of his $\backslash$ her time on weekdays at work and home.

\subsubsection{User speed}

User speed is a measured feature that is model as a uniform random variable with a defined average speed. Fig. 4.5.a and Fig. 4.5.b illustrate the speed heat map in $\mathrm{km} / \mathrm{hr}$ for each location square using data generated for two days, a weekday and 


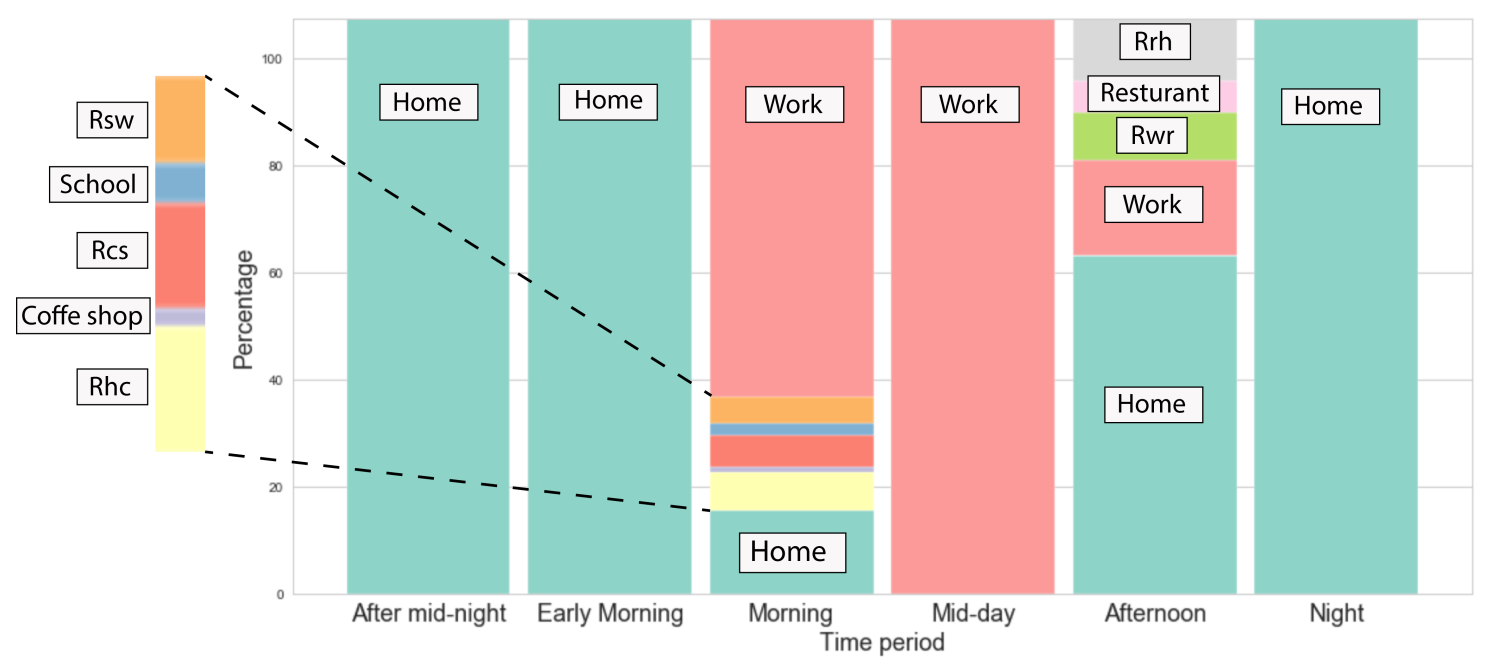

FiguRE 4.6: The percentage of time the user spent at each location over each time period on a weekday.

a weekend day, respectively. User speed in $\mathrm{km} / \mathrm{hr}$ is classified into four ranges: High, Medium, Low, and Zero speed to create the Speed range engineered feature.

\subsubsection{User activity}

Since user activity influences user satisfaction significantly, it is considered to be an essential part of the user context and incorporate it into the proposed dataset. A large body of literature discusses activity prediction using ML from data collected using various types of sensors [50]. Accordingly, instead of adding raw sensor measurements to the proposed dataset, Activity labels are incorporated as an engineered feature. Although there exist numerous types of activities, for the purpose of this discussion, it is assumed that the recorded user activity is one of the following: 1) walking, 2) sitting, 3) walking upstairs, 4) walking downstairs, 5) standing, and 6) laying. Similar to the aforementioned context variables, the user activity variable is designed such that it is correlated with other context variables. Fig. 4.7 presents the histogram of the recorded instances on a weekday for each of the six activities at two locations, home and work. It can be seen that the user's most frequent recorded activity at home is laying, whereas the user's activity at work is confined to three activities: sitting, walking, and standing.

\subsubsection{Request arrival time}

It is assumed that the user requests $N$ service requests in a day with at most one request within a TS. The arrival of a request during a certain instant is recorded to 


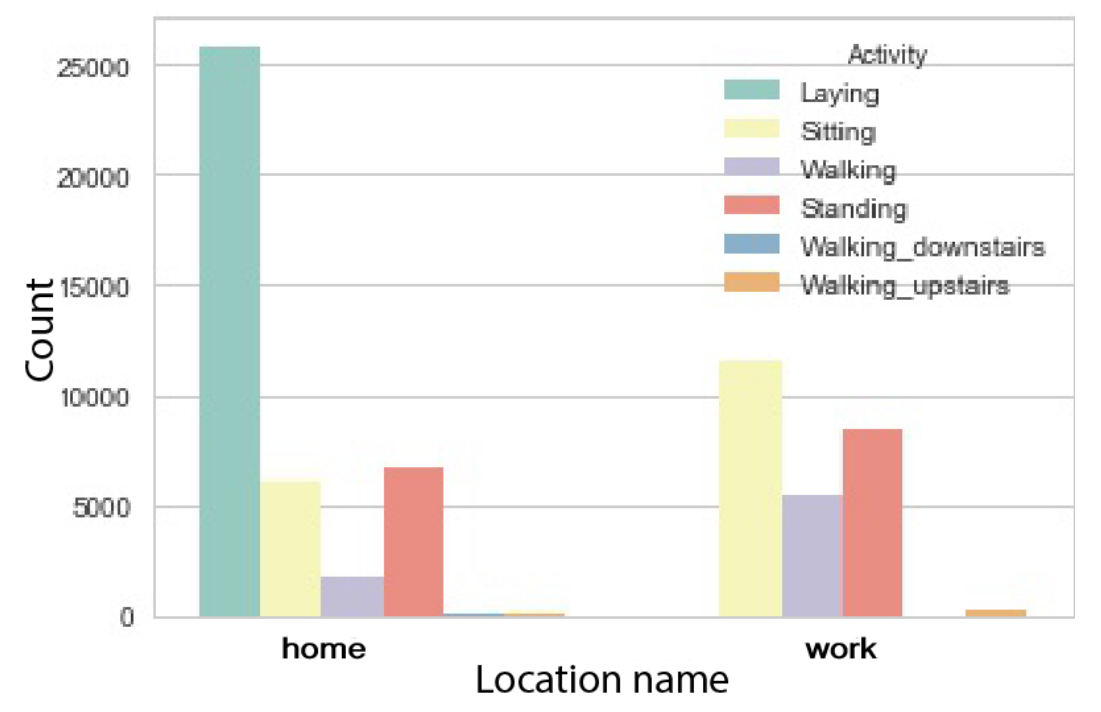

FiguRE 4.7: The histogram of the recorded instances on a weekday for the six activities at two locations.

the dataset using a binary variable called Request arrived (see Fig. 4.3). Request arrival is dependent on time $(t)$ in hours, the day of the week $(d)$, location $(l)$, and speed $(s)$ in $\mathrm{km} / \mathrm{hr}$. In order to model this dependency, a non-homogenous Poisson random process is used [51]. A non-homogeneous Poisson process with arrival rate $\lambda_{r}(t, d, l, s)$ is defined as a counting process $N(t)$ for $t>0$. The probability of a request arrival in an interval $\delta$ is given as:

$$
\begin{aligned}
& P\{N(t, t+\delta)=1\}=\delta \lambda_{r}(t, d, l, s)+o(\delta), \\
& P\{N(t, t+\delta) \geqslant 2\}=o(\delta),
\end{aligned}
$$

where $N(t, t+\delta)=N(t+\delta)-N(t)$ and $o(\delta)$ is a function that goes to 0 faster than $\delta$ as $\delta \Longrightarrow 0$. Since it is required to generate a correlation between the Request arrived variable and other context variables, $\lambda_{r}$ is modeled as a function of the context variables $t, d, l$, and $s$. In order to illustrate the correlation between the request arrival rate and other context variables, $d$ and $t$ are chosen as examples of features to plot $P\{N(t, t+\delta)=1\}$. Fig. 4.8 and Fig. 4.9 demonstrate $P\{N(t, t+$ $\delta)=1\}$ vs. $t$ for $\mathbf{a}$ ) a weekday and $\mathbf{b}$ ) a weekend day. For a weekday, the user tends to issue requests with a higher probability between 5AM and 3PM. By contrast, for a weekend day, the user tends to issue requests with a higher probability between $11 \mathrm{AM}$ and 5PM. 
Chapter 4. A Synthetic User Behavior Dataset Design for Data-driven AI-based Personalized Wireless Networks

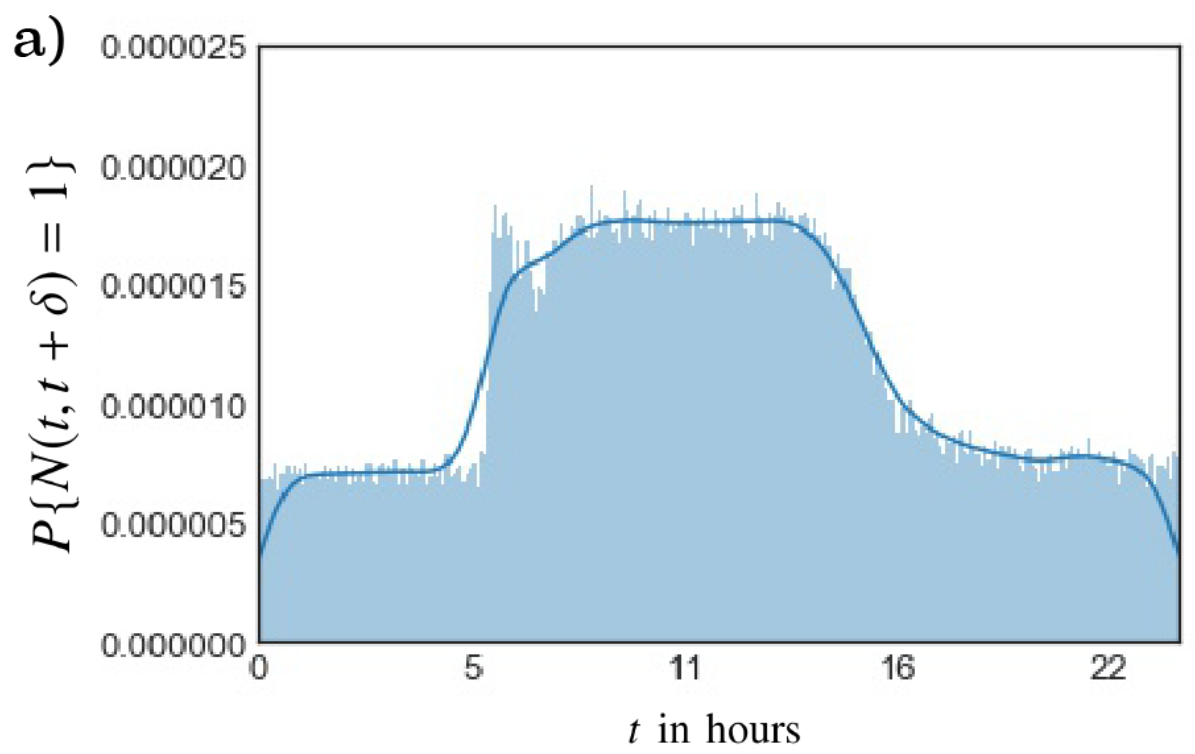

FIgURE 4.8: The probability of request arrival (i.e., $P\{N(t, t+\delta)=1\})$ vs. $t$ in hours for a weekday.

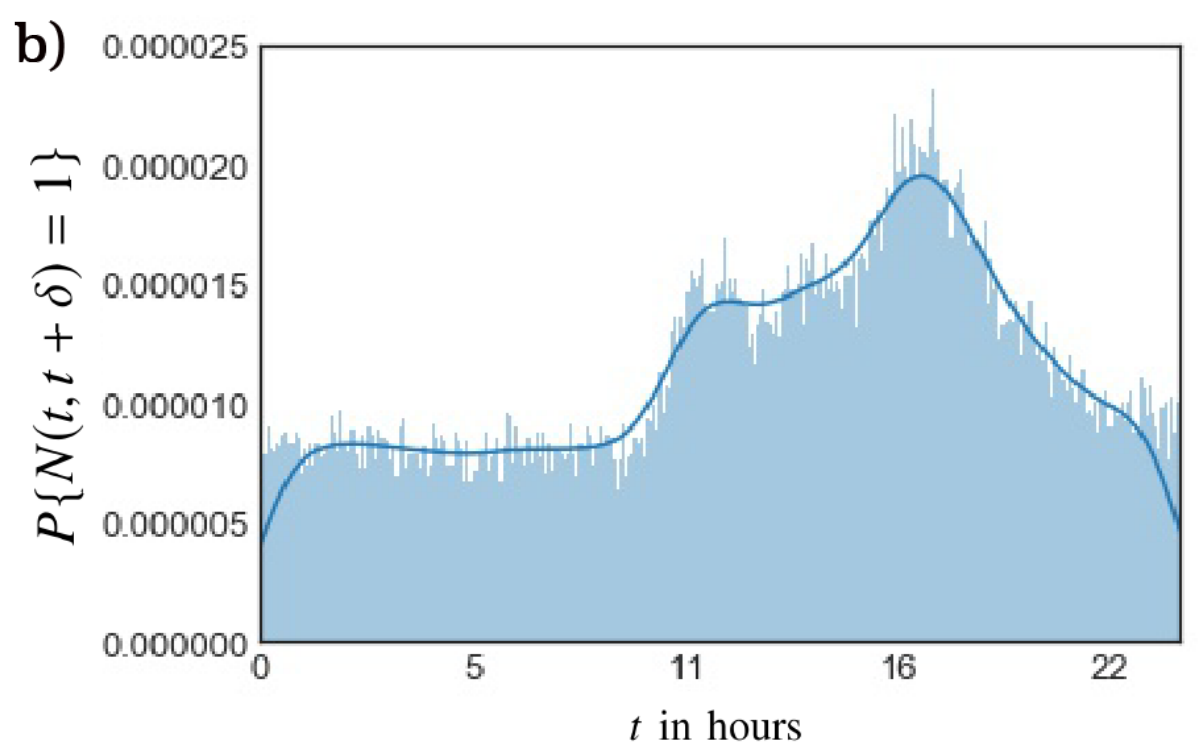

FIgURE 4.9: The probability of request arrival (i.e., $P\{N(t, t+\delta)=1\})$ vs. $t$ in hours for a weekend day.

\subsubsection{Application choice}

It is assumed that the user issues service requests from a specific set of applications (see Table 4.1). Similar to other context variables, the application feature is also correlated with other context variables, such as time and location, and it is recorded to the dataset under the Application feature. 


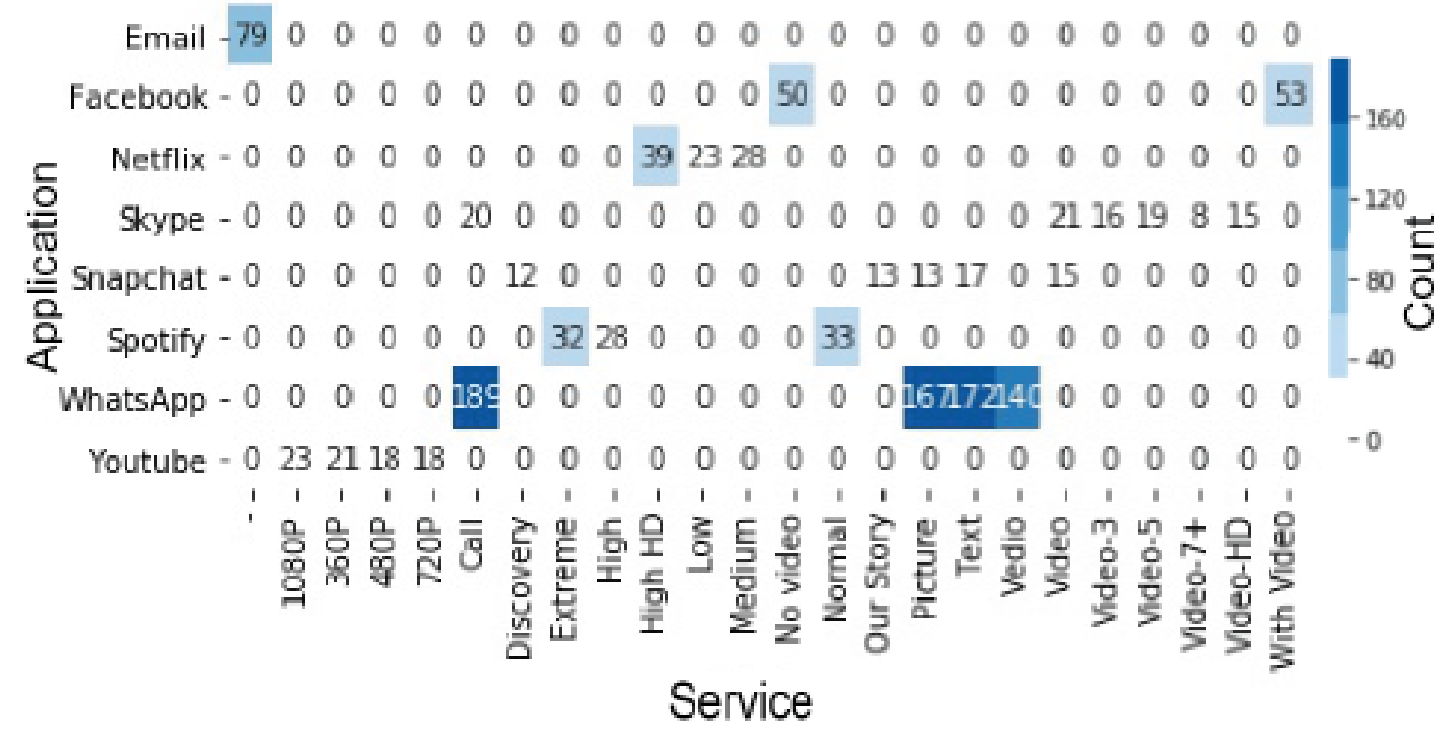

FiguRE 4.10: Heat map of the instance count for the requested services and the corresponding applications over a week.

\subsubsection{Service type}

Table 4.1 lists the set of services associated with each application. Similar to applications, the choice of the Service depends on the context as well. Fig. 4.10 depicts the heatmap of the instance count for services requested by the user and their corresponding application over a one-week period. As shown in Fig. 4.10, the most popular service for this user is WhatsApp video.

\subsubsection{Demand rate, given rate, and $\Delta$}

The Demand rate (i.e., $Q o S_{d}$ ) feature represents the rate requirement associated with the service requested by the user. Table 4.1 lists the service demand rate requirement data aggregated from several sources. On the other hand, the rate assigned to the user by the network is recorded to the dataset under the Given rate (i.e., $Q o S_{p}$ ) feature. Given rate is designed in such a way that it is correlated to some other context variables, such as time and location to model the effect of cell congestion. Lastly, for each service request, $\Delta$ is computed and recorded to the dataset under the Delta feature. 
Chapter 4. A Synthetic User Behavior Dataset Design for Data-driven AI-based Personalized Wireless Networks

TABLE 4.1: The set of services and their associated demand rate for the considered set of applications.

\begin{tabular}{|c|c|c|}
\hline Application & Service & Demand (in kbps) \\
\hline \multirow{4}{*}{ Yout } & $360 p$ & 93.75 \\
\hline & $480 p$ & 126 \\
\hline & $720 p$ & 312.5 \\
\hline & $1089 p$ & 562.5 \\
\hline \multirow{3}{*}{ (I) } & Low & 38.33 \\
\hline & Medium & 193.3 \\
\hline & $H D$ & 833.3 \\
\hline \multirow{2}{*}{ (f) } & No video & $25-41$ \\
\hline & With video & $93.75-125$ \\
\hline \multirow{3}{*}{ (2) } & Normal & 96 \\
\hline & High & 160 \\
\hline & Extreme & 320 \\
\hline (3) & - & $25-41$ \\
\hline \multirow{4}{*}{ (2) } & Text & 71 \\
\hline & Picture & 867 \\
\hline & Video & 125 \\
\hline & Call & 740 \\
\hline \multirow{5}{*}{ (3) } & Text & 17.3 \\
\hline & Picture & 86.7 \\
\hline & Video & 89.1 \\
\hline & Our story & 14.76 \\
\hline & Discovery & 90 \\
\hline (1) & - & $16.67-166.67$ \\
\hline \multirow{6}{*}{ (S) } & Voice & 100 \\
\hline & Video & 500 \\
\hline & Video HD & 1500 \\
\hline & Video-3 people & 2000 \\
\hline & Video-5 people & 4000 \\
\hline & Video-5 people & 8000 \\
\hline
\end{tabular}




\subsubsection{User satisfaction prediction experiments using the proposed dataset design}

In practice, the relationship between satisfaction and $\Delta$ (e.g., Fig. 3.4 and 3.5) is not available to the network. Besides, user satisfaction is shaped by a complex set of factors and variables in which their interconnected effects on user satisfaction cannot be modeled using traditional mathematical equations. Therefore, ML and data analytics is important to extract patterns and knowledge from historical data and use it to predict user satisfaction behavior in the future.

For the purpose of illustrating the benefits of the proposed ZoT model and the dataset, user satisfaction prediction is performed using the following traditional set of ML algorithms: Decision Tree (DT), K-nearest neighbor (Knn), and Random Forest (RF). Data preprocessing, such as filtering, scaling, feature selection, and addressing class imbalance is considered. Moreover, hyperparameter tuning is conducted using grid-search. Fig. 4.11 depicts the 10 -folds cross-validation prediction accuracies for each ML algorithm. Since there are six satisfaction levels, the random choice accuracy level is 0.166. Fig. 4.11 shows that the best performance is achieved using RF (ensemble) algorithm with an average accuracy of 0.884 compared to 0.85 for DT and Knn. It is worth mentioning that the predictors' performance should be directly related to the number of relevant context variables available to the ML algorithms.

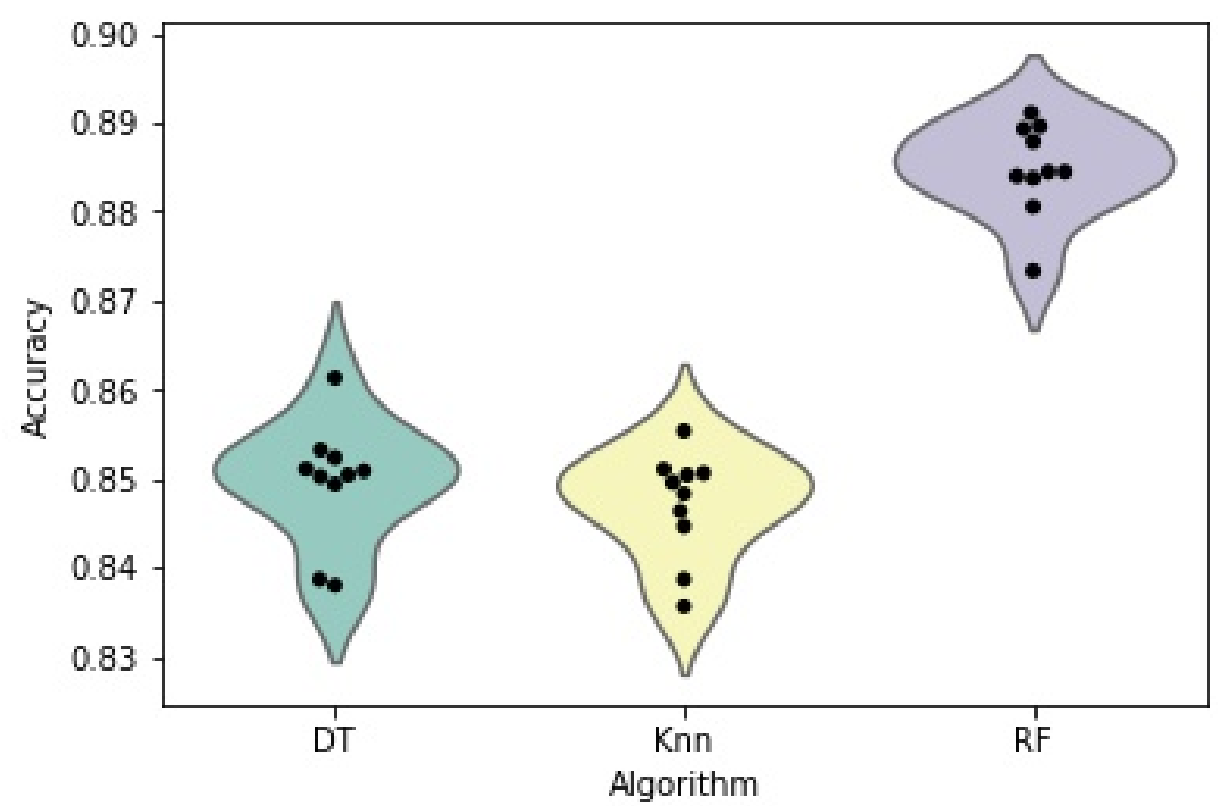

FiguRE 4.11: Accuracy swarm plot for 10-folds cross-validation using DT, Knn, and RF algorithms. 


\subsection{Integrating Realistic Characteristics into the Designed Dataset}

\subsubsection{Clustering users into personas}

Although the tree data generator proposed in this document could be used to create almost an infinite number of datasets with unique and different behavioral patterns, in the real world, people share certain similarities. For example, students tend to be in school during daytime hours on weekdays; likewise, professionals generally work during the same hours. Therefore, it should not be assumed that all people have fundamentally different behavioral patterns. Grouping people with similar behavioral patterns can also make processing their data more efficient, and it can improve the accuracy of predicting future user satisfaction levels. In this work, the TG structure of users with similar behavioral patterns is referred to as a "user persona". A persona-specific dataset is created by feeding the persona's parameters as input into the TG generator. Identical TG input parameters mean that people belonging to the same persona have identical $\mathcal{A}$ and $\mathcal{B}$ matrixes, which indicates that they will have similar distributions and patterns within their data.

\subsubsection{Integrating real features from real sensor sata}

Integrating errors into synthetic datasets is a fundamental requirement for creating datasets with realistic characteristics. Different datasets have different types of errors depending on several factors, such as the utilized data collection method and data type. One strategy that can be used to add errors to datasets is the integration of real data measurements with inherent errors and noise. In this section, real data are integrated from the sensor measurements dataset available in [50]. The authors in [50] carried out an experiment to collect data for a group of 30 volunteers with ages ranging from 19 to 48 years in order to obtain a dataset of phone sensor data labeled with ground truth activity labels. The activity labels were the following: standing, sitting, laying down, walking, walking downstairs, and walking upstairs. Sensor data are collected from sensors, such as body acceleration, gravity acceleration, and body angular speed sensors. Data pre-processing, including filtering, sampling, and normalization, has been already applied to the raw sensor data. The authors in [50] also engineered the sensor data features to create more relevant and meaningful features. In this work, the context-activity dataset in [50] is referred to as the Human Activity (HA) dataset, whereas the 


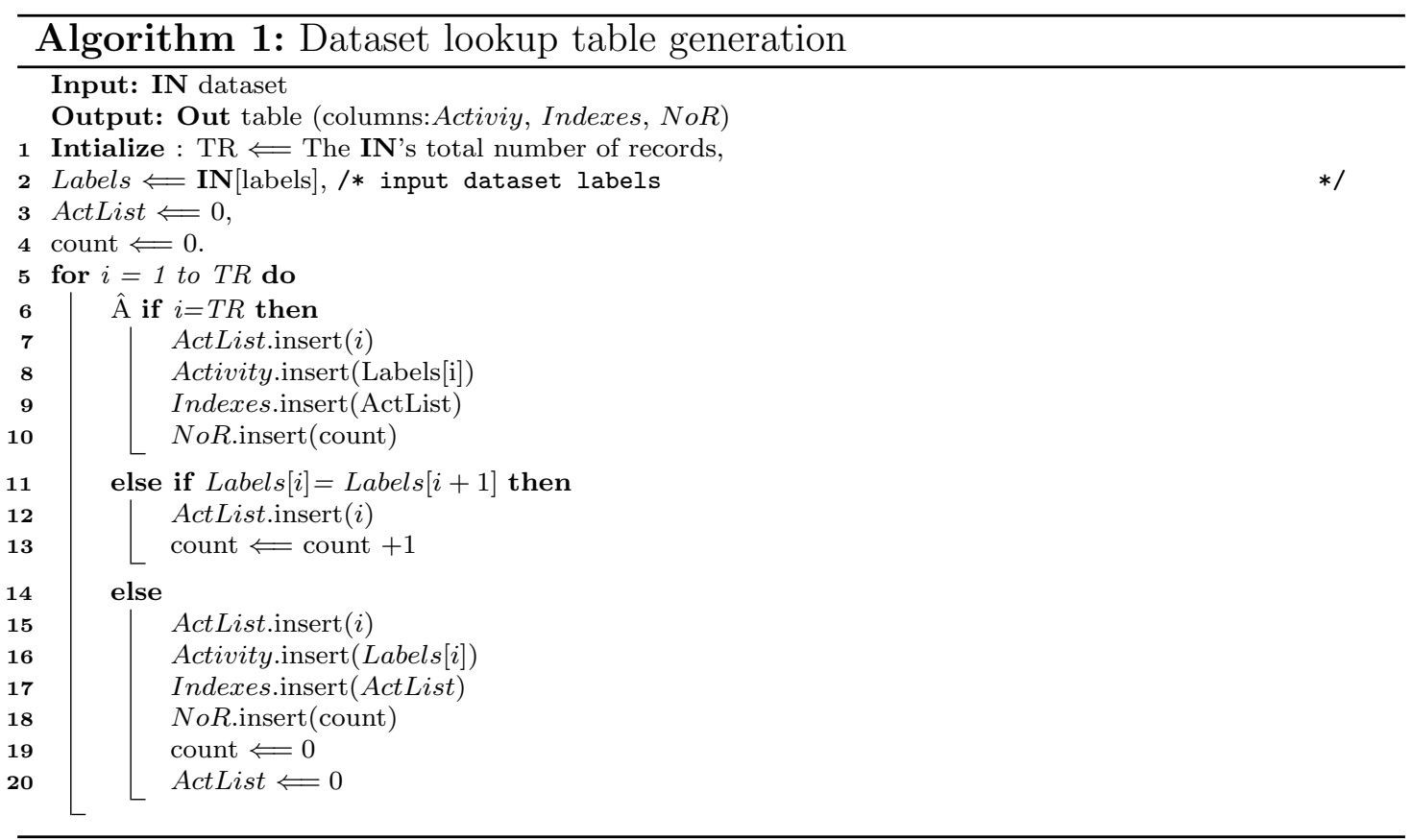

datasets proposed in this document are referred to as the user context (UC) datasets. The process of integrating HA into UC involves the following steps:

- Create a lookup table for HA dataset called HAL. HAL has three columns: Activity, Indexes, and Number of Records (NoR). Each row is called a record. One record is created for each consecutive set of HA records that have the same activity label. The indexes (i.e., row numbers) of those records are recorded under the Indexes column and the number of HA records in each set is recorded under the NoR column. Algorithm 1 describes the process of creating the lookup table HAL.A ${ }^{1}$

- Similar to HAL, create a lookup table for UC dataset called UCL. This lookup table is created using Algorithm 1 as well and it has three columns: Activity, Indexes, and NoR.

- Augment sensors data in UC dataset by data in HA dataset using Algorithm 2 .

\footnotetext{
${ }^{1}$ Bold variables represent tables (or datasets) and italic variables represent arrays. $\mathbf{H A}[$ Activity $][\mathrm{i}]$ represents the value in dataset $\mathbf{H A}$, at column Activity and record number $i$.
} 


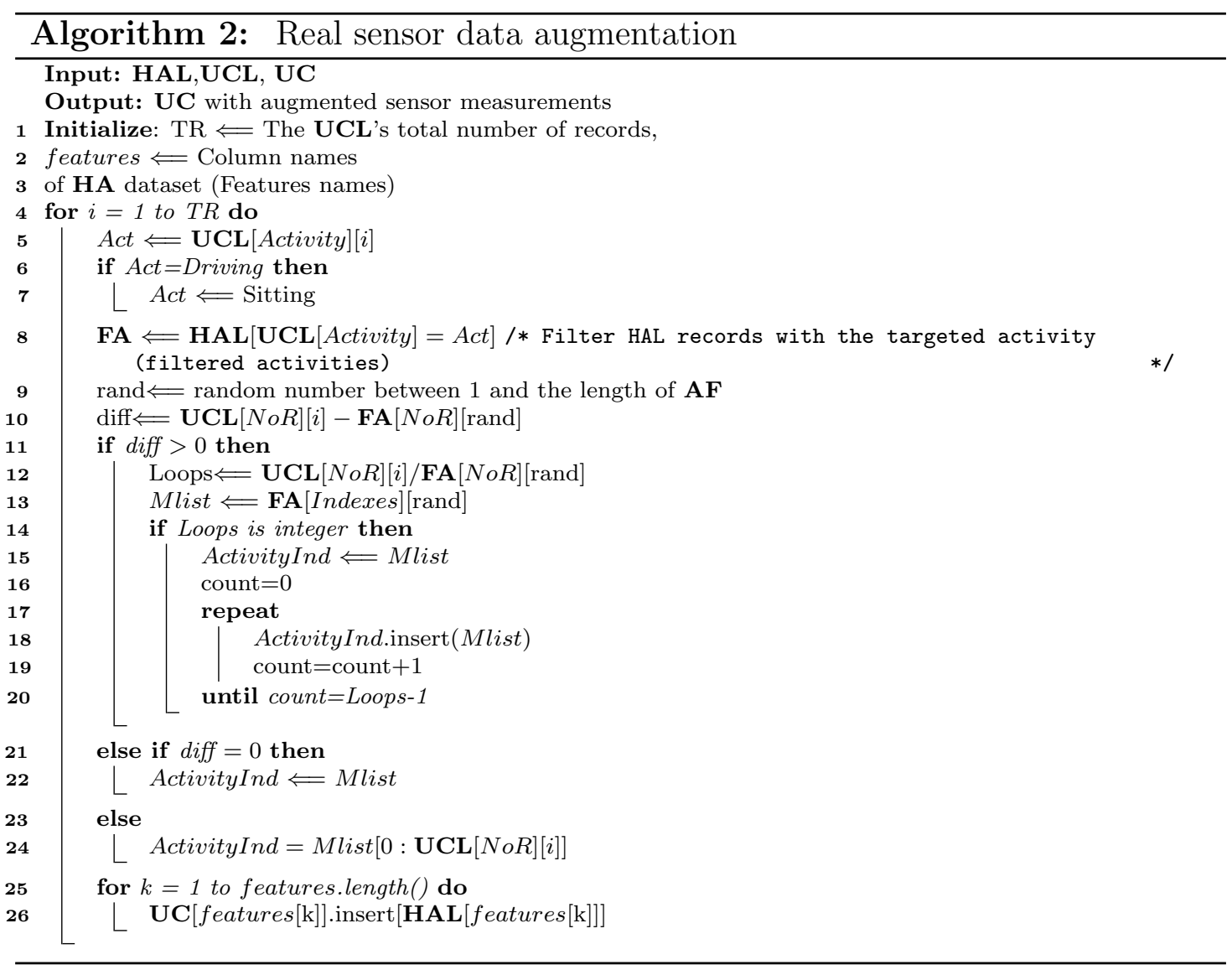

\subsubsection{User satisfaction uncertainty modeling}

User satisfaction depends on many variables some of which are difficult to track and measure. Fig. 4.12 visualizes the problem of user satisfaction prediction from user context data. As shown in Fig. 4.12, all the variables affecting user satisfaction prediction accuracy at a certain context are visualized as a plane. Each plane is associated with a certain context and user satisfaction behavior (i.e., mapper). User satisfaction behavior in each context is dependent on all context variables in the context plane. Missing variables in the context plane will create gaps, which will add noise to the associated user satisfaction mapper as a result and therefore hinder the accurate prediction of user satisfaction. It is worth noting that the accuracy of user satisfaction prediction is highly correlated with the number of missing variables and the amount of missing information. In order to integrate the resulting error from missing user context information, uncertainty is incorporated into the user satisfaction mapper. Satisfaction uncertainty implies that for the same context and value of $\Delta$, satisfaction might slightly vary due to a shift in the predicted $Z o T$ behavior compared to the original behavior. For the purpose of this work, it is assumed that the changes in $Z o T$ s for the same context 


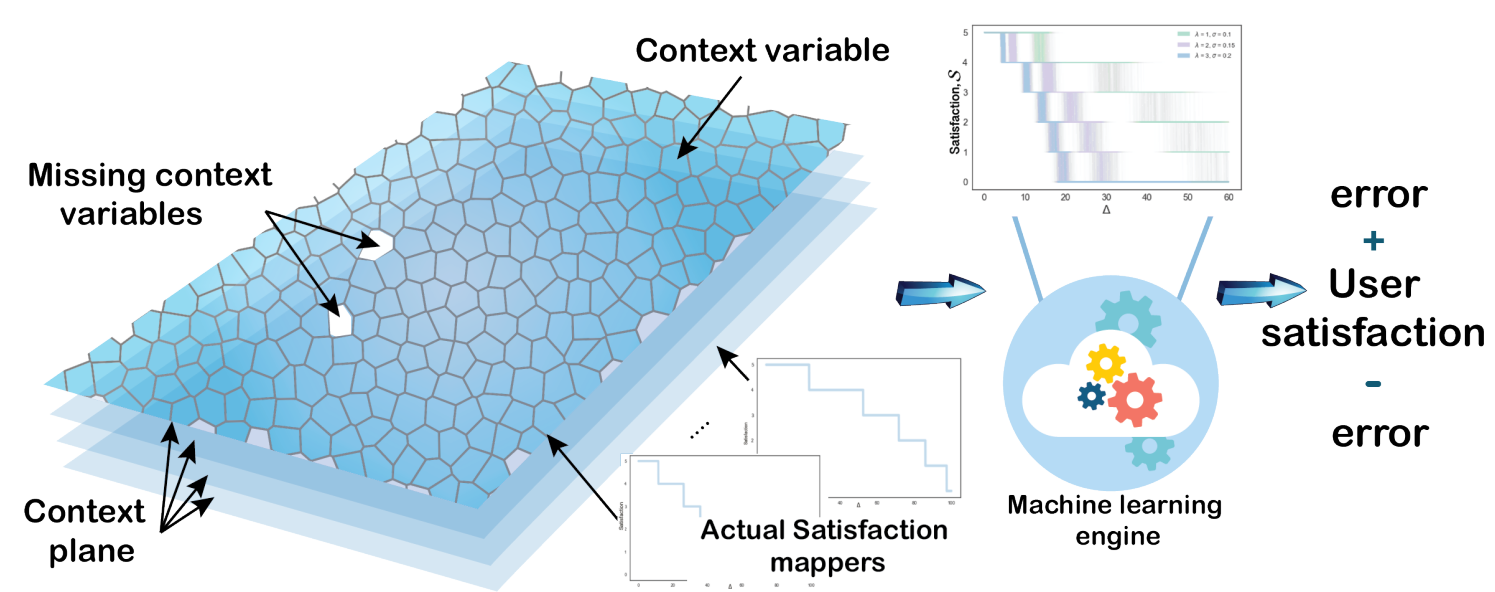

FiguRE 4.12: The problem of user satisfaction prediction from user context data.

and $\Delta$ is due to a shift in $\lambda$ value of the predicted satisfaction behavior. The value of $\psi$ is assumed to be fixed for specific context values. The change in $\lambda$ is modeled by an additional error $\delta$ added to the original $\lambda$ value associated with the considered context. In this work, the error $\delta$ is assumed to have a normal probability distribution as follows:

$$
P(\delta)=\frac{1}{\sqrt{2 \pi \sigma^{2}}} e^{-\frac{\delta^{2}}{2 \sigma^{2}}} .
$$

Hence, the final $\lambda$ with added error $\left(\lambda_{e}\right)$ is given by

$$
\lambda_{e}=\lambda+\delta
$$

The values of $\lambda$ and $\sigma$ are considered as inputs to the TG generator. This will enable the TG generator to associate a certain satisfaction behavior and error level with each context. Fig. 4.13 visualizes the satisfaction mapper in (3.1) with added uncertainty error. It can be seen that as $\sigma$ increases, the amount of error added to user satisfaction decreases.

\subsubsection{User satisfaction prediction experiments using the proposed dataset with integrated realistic character- istics}

As mentioned earlier, the relationship between satisfaction and $\Delta$ is not available to the network. Besides, user satisfaction is dependent on a complex set of factors, variables, noise, and other uncertainties. In order to capture the inherent 


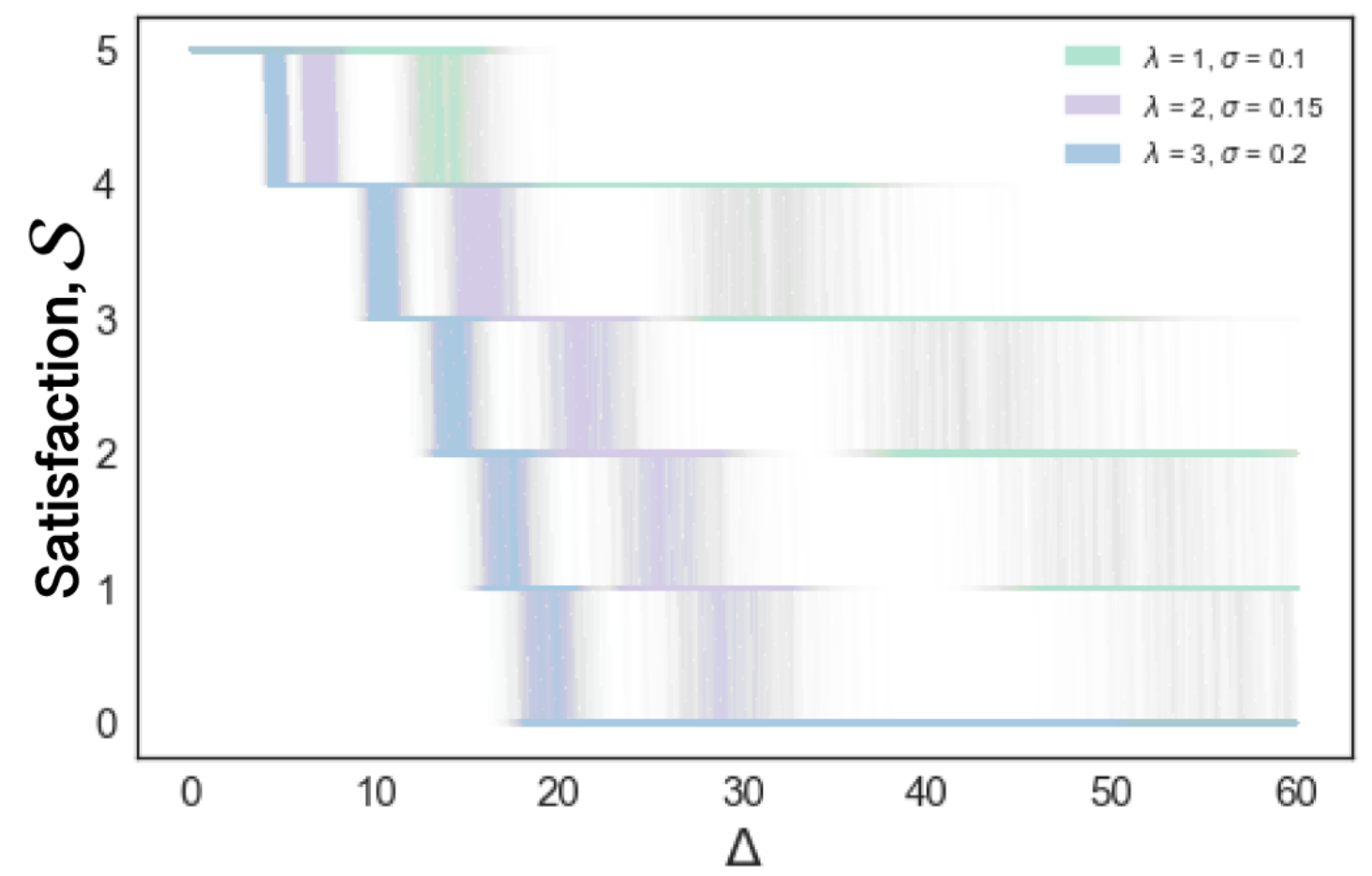

FiguRE 4.13: Satisfaction mapper with added uncertainty for $\psi=h ; \sigma=0.1$, 0.15 , and 0.2 ; and $\lambda=1,2$, and 3 .

patterns and anticipate future user satisfaction values from the available context data, machine learning and big data analytics are essential. In order to investigate the effect of augmenting real sensor data into the dataset and the effect of incorporating statistical noise in the satisfaction mappers, four classification experiments are performed to predict user satisfaction using the following datasets:

- A dataset with noise free satisfaction and without real sensor measurements augmentation. The accuracies for the three algorithms are labeled DT, Knn, and RF.

- A dataset with noisy satisfaction and without real sensor measurements augmentation. The accuracies for the three algorithms are labeled DT-n, Knn-n, and RF-n.

- A dataset with noise-free satisfaction and real sensor measurements augmentation. The accuracies for the three algorithms are labeled DT-a, Knn-a, and RF-a.

- A dataset with noisy satisfaction and real sensor measurements augmentation. The accuracies for the three algorithms are labeled DT-na, Knn-na, and RF-na. 


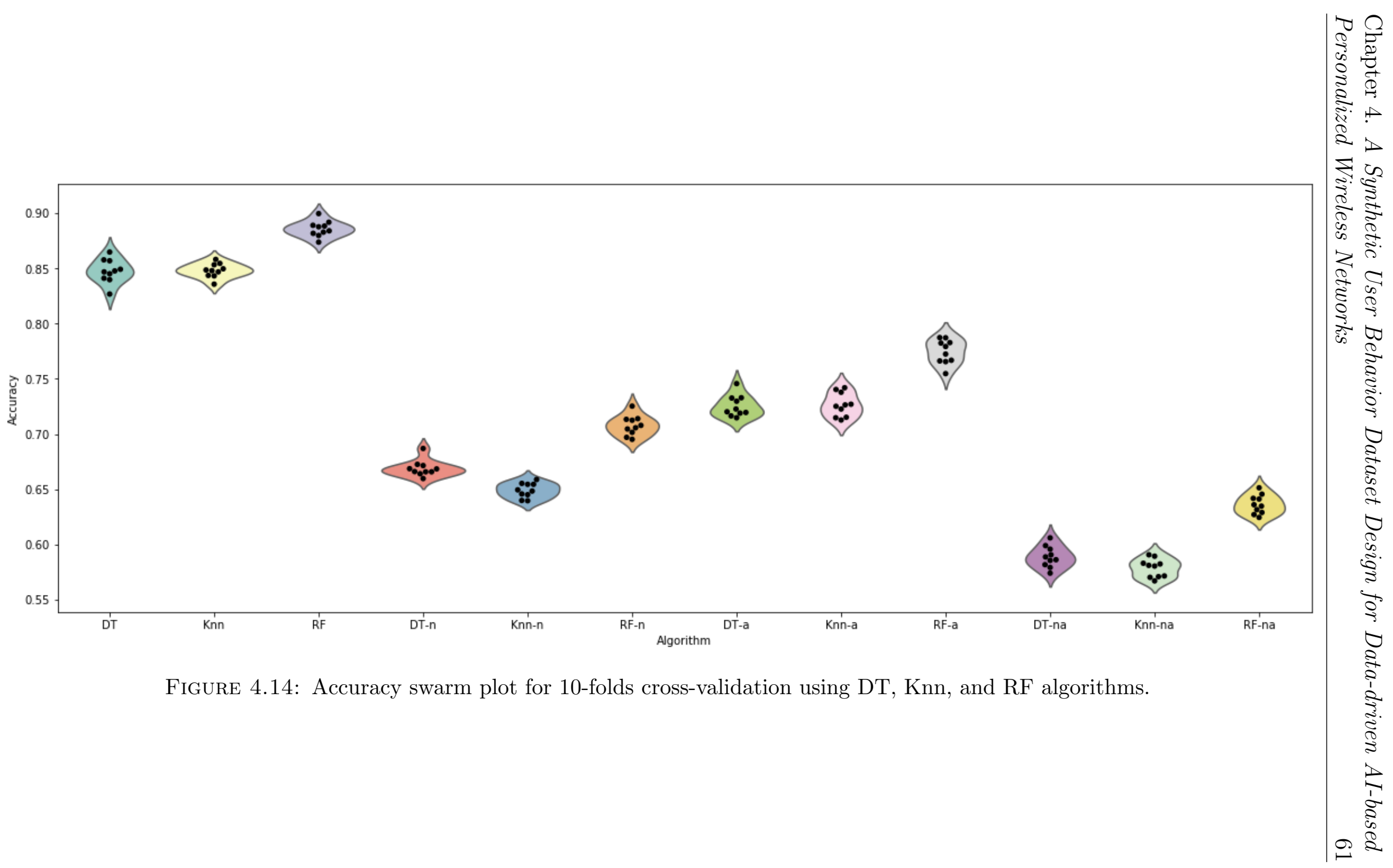


All the aforementioned experiments are performed on the working professional persona dataset using the following set of ML algorithms: Decision Tree (DT), K-nearest neighbor (Knn), and Random Forest (RF). Data preprocessing, such as filtering, scaling, feature selection, and addressing class imbalance is considered. Moreover, hyperparameter tuning is conducted using grid-search.

Fig. 4.14 depicts the 10-folds cross-validation prediction accuracies for the four experiments and for each ML algorithm. Since there are six satisfaction levels, the random choice accuracy level is 0.166 . It can be seen that the best accuracies were achieved by the first experiment, which is expected since this experiment was performed on error-free data. By contrast, the fourth experiment had the worst accuracies of all experiments due to the errors of the added satisfaction uncertainty and the real sensor measurements. It is worth noting that the predictors' performance in experiments three and four rely on the amount of noise added to the satisfaction mappers (i.e., the value of $\sigma$ ). 


\section{Chapter 5}

\section{User Persona Design for Personalized Wireless Networks}

In this chapter, a novel big data-driven framework to predict user persona type in personalized wireless networks is proposed. The presented framework is supported by a confidence assessment step to increase the accuracy of predictions and ensure the association of the correct persona type with each user. Also, a comprehensive study is conducted to investigate the effects of different amounts of data and confidence levels on the performance of the overall framework. Finally, using a simulated personalized wireless network, the behavior of different personas are compared in terms of the amount of saved resources and achieved satisfaction levels. 
Wireless network personalization is an emerging technology that has considerable potential to achieve the ultimate balance between resource allocation and user satisfaction. One of the main enablers of the proposed framework in Chapter 2 is the continuous monitoring and prediction of dynamic user satisfaction levels in various contexts. Accurate satisfaction prediction requires huge amounts of data, and unfortunately, data and the process of acquiring it are expensive. Nonetheless, a closer look at user behavior and satisfaction levels reveal that certain users share certain similarities. Capitalizing on these similarities, this chapter introduces the concept of user persona in personalized wireless networks. A persona in a personalized wireless network is a set of user context patterns and their associated user satisfaction behavior characteristics that are shared by a group of people. Associating users with pre-existing user personas will enable networks to provide highly personalized service with a minimal amount of data, thereby improving the efficiency of personalized networks. Fig. 5.1 illustrates the difference between persona design in marketing and social media contexts and personas in personalized wireless networks. The term user persona in the marketing literature refers to shared behavioral characteristics and activity patterns among a group of people. On the other hand, as shown in Fig. 5.1, the concept of user persona in personalized wireless networks refers to a shared user ZoT satisfaction behavior at similar contexts among a group of people. In order to build the aforementioned capabilities, and to integrate the benefits of user persona design into personalized wireless networks, an innovative big data-driven framework is proposed. The framework employs real-time data analytics and ML capabilities to learn and predict user personas in real-time, which subsequently improves user satisfaction monitoring and prediction in personalized wireless networks.

\subsection{Background}

Studying, designing, constructing, and predicting user personas for various applications has considerable potential and benefits. In this section, the literature on user personas is categorized in terms of the types of applications utilizing user personas, user persona design, sources of data utilized to create user personas, and methodologies used to classify users/people into personas. 

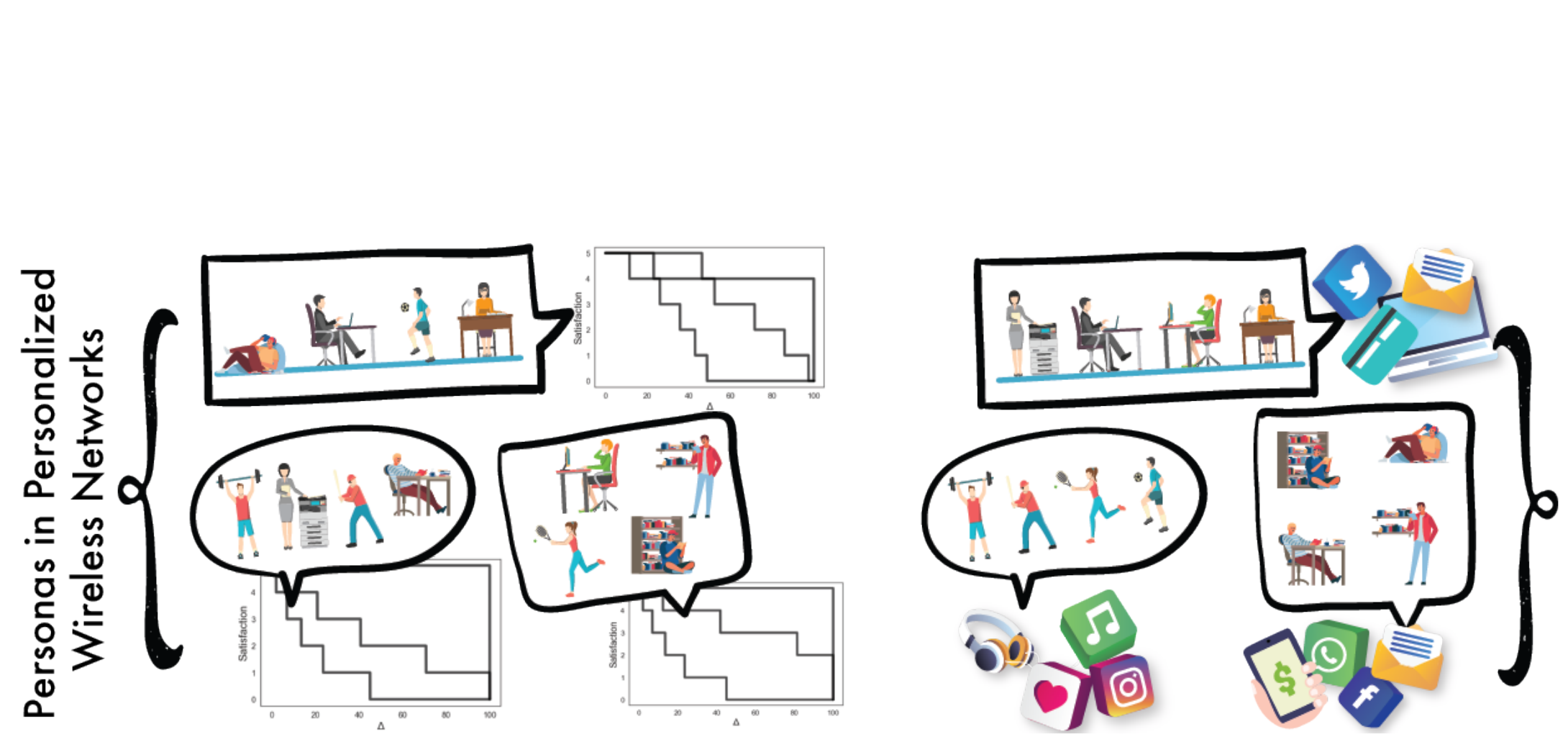

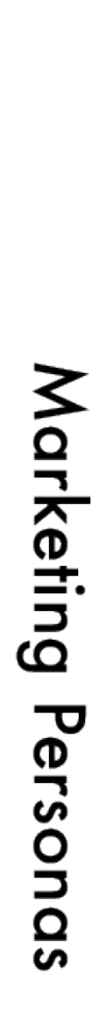

FiguRE 5.1: A comparison between persona design in the literature and the proposed persona design for personalized networks. 


\subsubsection{Types of applications utilizing user personas}

The concept of user personas features prominently in business, advertising, and marketing studies for the purpose of abstract user representations [52]. Advertisements and marketing strategies based on user personas are very popular in the literature, and many solutions and intellectual properties have been proposed/registered to actualize this approach $[53,54]$. Another application of user personas lies in providing customized and personalized services, such as call center services [55]. In addition, personas have been utilized to capture the individual characteristics of speech and background [56]. Finally, the concept of personas has also been proposed to evaluate people in terms of performance efficiency and to evaluate their qualifications to do specific tasks $[57,58]$.

\subsubsection{User persona design}

The user persona designs proposed in the literature can be categorized into two main categories. The first design category involves personas with fixed characteristics designed by domain experts. For instance, the author in [59] proposed a statistical technique to create user personas based on user needs and preferences. The second category involves flexible personas with dynamic characteristics created using big data-driven real-time analytics, such as the solutions proposed in [60] and [61].

\subsubsection{Sources of data}

Depending on the purpose of the persona design and the targeted application, several sources of data have been utilized to collect the information required to group and categorize users/people. This section categorizes them as offline and online data sources. Offline data are manually collected using studies and questionnaires (e.g., market segmentation studies, field studies, and focus groups) [59]. By contrast, online data are collected from the activities of users on the cloud. For instance, the authors in [61] utilized social media data, specifically youtube social media, to create user personas.

\subsubsection{Persona classifications/clustering approaches}

Researchers have adopted several approaches for classifying users into different personas. One of the most popular approaches is to cluster or classify users based 
on behavioral data, such as physical activity and virtual activities (e.g., websites visited and clicks.). Another approach is to utilize information about user interests, opinions, age, and other demographic information [59]. Moreover, personas are also grouped based on user goals and decision-making preferences [62]. It is worth noting that some approaches are designed using a combination of some or all of the aforementioned approaches [60, 61].

\subsection{Benefits of Utilizing User Persona in User Satisfaction Predcition}

There are several benefits to integrating persona modeling in the prediction of user satisfaction values. First of all, in the real world, people share certain similarities. Therefore, predicting user satisfaction based on the assumption that all users in the network are drastically different is not an efficient or practical design. Grouping users with similar contextual and user satisfaction behavioral characteristics reduces the need for collecting and storing huge amounts of data; it also reduces the computational complexity and processing power required. Another notable advantage of integrating user persona modeling is that it enables personalized wireless networks to provide personalized services to new users joining the network about whom there is not yet enough information concerning their preferences or behavior in the network. This can be done by simply associating the new user with a pre-existing user persona.

\subsection{User Persona Design}

In this chapter, four distinct user personas are proposed: 1) a working professional, 2) a high school student, 3) a university student, and 4) a homemaker. Not only do people with similar personas share similar behavior patterns, but the correlation between their context and satisfaction data is similar as well. Table 5.1 lists the four personas and their corresponding ring of locations for both weekdays and weekends. Locations grouped in one bracket indicate that the user can go to either one with a certain probability. The four datasets generated for the four personas are available in [39]. 
TABLE 5.1: Weekdays and weekends location rings for the four user personas.

\begin{tabular}{|c|c|c|}
\hline Persona & Weekday ring of locations & Weekend ring of locations \\
\hline Working professional & $\begin{array}{c}\text { Home } \Rightarrow R_{h c} \Rightarrow \text { Coffee shop } \Rightarrow R_{c s} \Rightarrow \text { School (drop kids) } \\
->R_{s w} \Rightarrow \text { Work } \Rightarrow R_{w r} \Rightarrow \text { Restaurant } \Rightarrow R_{r h} \Rightarrow \text { Home }\end{array}$ & $\begin{array}{c}\text { Home } \Rightarrow R_{h s} \Rightarrow \text { Shopping mall } \Rightarrow R_{s p} \Rightarrow \\
\text { Park } \Rightarrow R_{p h} \Rightarrow \text { Home }\end{array}$ \\
\hline High school student & Home $\Rightarrow R_{h s} \Rightarrow$ School $\Rightarrow R_{s h} \Rightarrow$ Home & $\begin{array}{c}\text { Home } \Rightarrow R_{h-p m} \Rightarrow\{\text { Park, Movie }\} \\
\Rightarrow R_{p m-h} \Rightarrow \text { Home }\end{array}$ \\
\hline University student & $\begin{array}{c}\text { Home } \Rightarrow R_{h c} \Rightarrow \text { Coffee shop } \Rightarrow R_{c u} \Rightarrow \text { University } \\
\Rightarrow R_{u-l g} \Rightarrow\left\{\text { Library, GYM } \Rightarrow R_{l g-h} \Rightarrow \text { Home }\right.\end{array}$ & $\begin{array}{c}\text { Home } \Rightarrow R_{h g} \Rightarrow \text { GYM } \Rightarrow R_{g-p f} \Rightarrow \\
\text { Park, Friends house }\} \Rightarrow R_{p f-h} \Rightarrow \text { Home }\end{array}$ \\
\hline Homemaker & $\begin{array}{c}\text { Home } \Rightarrow R_{h s} \Rightarrow \text { School (drop kids) } \Rightarrow R_{s c} \Rightarrow \text { Coffee shop } \\
\left.\text { (meet friends) } \Rightarrow R_{c s} \Rightarrow \text { School (pick up kids }\right) \Rightarrow R_{s h} \Rightarrow \text { Home }\end{array}$ & $\begin{array}{c}\text { Home } \Rightarrow R_{h s} \Rightarrow \text { Shopping mall } \Rightarrow R_{s p} \Rightarrow \\
\text { Park } \Rightarrow R_{p h} \Rightarrow \text { Home }\end{array}$ \\
\hline
\end{tabular}




\subsection{Big Data-Driven Persona Prediction Frame- work for Personalized Wireless Networks}

As mentioned earlier, users are generally classified on the basis of patterns in their behavior, activities, and interests. In personalized wireless networks, users are classified on the basis of patterns in satisfaction-context behavior. A satisfaction behavior for a user represents his/her satisfaction patterns for various levels of service performance in a specific context. Integrating the capabilities of identifying these behavioral and satisfaction patterns is key for enabling personalized wireless networks. In Fig. 5.2, a big data-driven framework is proposed for implementing user persona prediction in personalized wireless networks. The proposed framework consists of three main processes: development, deployment, and production. In this section, these three processes and the steps involved in each are discussed.

\subsubsection{Model development}

As shown in Fig. 5.2, the development process goes through multiple stages before the output model is deployed in the network. Here, the steps involved in the development process are described.

\subsubsection{Data collection and labeling}

The first stage is to collect context data and associated user satisfaction data from the network itself or from sensors. Both context and satisfaction levels data are combined into a labeled user context-satisfaction behavior dataset. Since such data is not publicly available due to privacy and confidentiality concerns, Chapter 4 presented synthetic context-satisfaction datasets that can be utilized for big data-driven prediction and optimization in personalized wireless networks. The datasets are designed meticulously to reflect realistic characteristics of real user data, and they are publicly available in a GitHub repository [39].

\subsubsection{Clustering context-satisfaction user data into personas}

The next step is to cluster users into personas based on patterns in their contextsatisfaction behavior captured by the collected data. Several unsupervised clustering algorithms can be utilized in this process, such as K-Means clustering. Then, the dataset for each user is labeled with a persona type. The utilized dataset in this chapter includes both user satisfaction information and persona type. The 


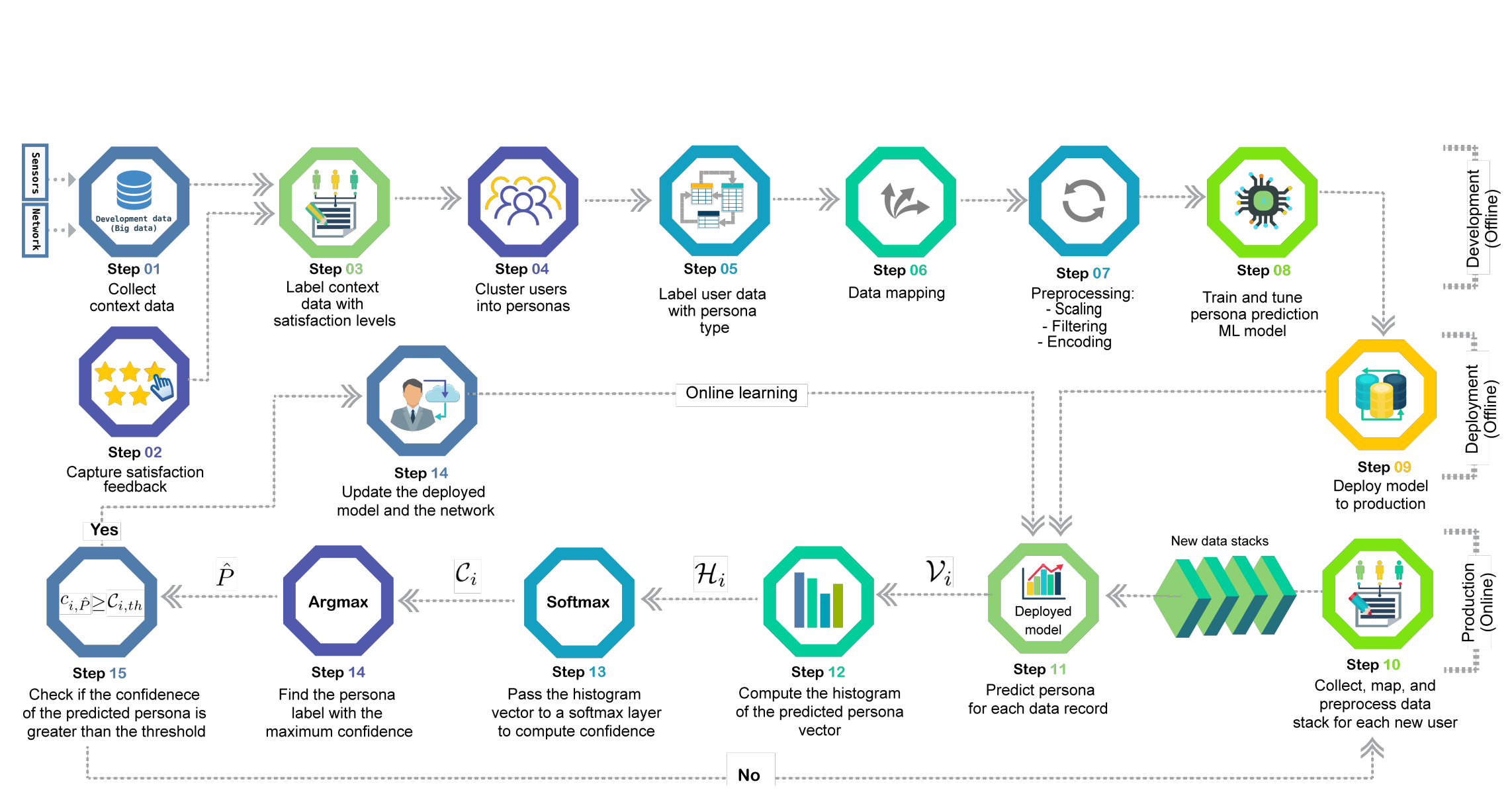

FiguRE 5.2: The proposed big data-driven persona prediction framework for personalized wireless networks. 
proposed datasets belong to four distinct personas: 1) a Working Professional (WP), 2) a High School Student (HS), 3) a University Student (US), and 4) a Homemaker (HM). For the purpose of this chapter, the four datasets are merged, which is designated as the User Context-Satisfaction (UCS) dataset. In addition, in order to distinguish between data associated with each persona, a new label column called Persona is incorporated. Table 5.2 shows the features of the dataset and an example of their values ${ }^{1}$.

\subsubsection{Data mapping}

In order to capture correlations between users belonging to the same persona, data should be mapped to shared space. For instance, user location is recorded as GPS coordinates. However, generally, user satisfaction behavior and persona types are actually correlated to a particular type of location (e.g., home) rather than to GPS coordinates. Therefore, the features in the UCS dataset are mapped to the same shared space.

\subsubsection{Preprocessing}

The next stage is to preprocess the data, which consists of the following steps.

Filtering. The UCS dataset was recorded for a period of one year. Also, the resolution (i.e., the time period between two records) of the UCS dataset is one second. For the purpose of this chapter, a part of the dataset is selected, where the filtered dataset spans a four-month period. Naturally, users do not use the network during all measuring instances. Therefore, since user satisfaction behavior is a crucial part of user persona design and prediction, records that had no service requests from the network and that did not carry any satisfaction information are filtered out. The dataset was filtered on the basis of the Request arrived feature, which is set to 1 when a user requests a service from the network and, otherwise, is set to 0 . The filtered number of records is 38,166 .

Data re-scaling. Scaling data is a very important step especially for ML algorithms, such as support vector machine and neural networks, where the distance between the data points matters. In this chapter, MinMax scaling is performed, which is typically done via the following equation:

$$
\mathcal{X}_{\text {scaled }}=\frac{x_{i}-\min (\mathcal{X})}{\max (\mathcal{X})-\min (\mathcal{X})}, \forall x_{i} \in \mathcal{X},
$$

\footnotetext{
${ }^{1}$ The dataset in [39] has other features, such as real sensor measurements. However, for the purpose of this chapter, only the features listed in Table 5.2 are considered.
} 
TABLE 5.2: Features of the UCS dataset.

\begin{tabular}{|c|c|c|c|}
\hline \hline No. & Feature name & Units & Example \\
\hline \hline 0 & Date & - & $2018-01-10$ \\
\hline 1 & Time & - & $14: 55: 02$ \\
\hline 2 & Day & - & Wednesday \\
\hline 3 & Classified days & - & Weekday \\
\hline 4 & Time period & - & Afternoon \\
\hline 5 & Location & - & {$[22,73]$} \\
\hline 6 & Location name & - & Work \\
\hline 7 & Speed & $\mathrm{km} / \mathrm{hr}$ & 5.2 \\
\hline 8 & Speed range & - & Low \\
\hline 9 & Activity & - & Walking \\
\hline 10 & Request arrived & - & 1 \\
\hline 11 & Application & - & WhatsApp \\
\hline 12 & Service & - & Picture \\
\hline 13 & Demand rate & $\mathrm{kbps}$ & 867 \\
\hline 14 & Min rate & $\mathrm{kbps}$ & 600 \\
\hline 15 & Given rate & $\mathrm{kbps}$ & 802 \\
\hline 16 & $\Delta$ & - & 65 \\
\hline 17 & Max $\Delta$ & - & 267 \\
\hline 18 & Satisfaction & - & 4.0 \\
\hline 17 & Persona & - & WP \\
\hline & & & \\
\hline
\end{tabular}

where $\mathcal{X}=\left[x_{1}, x_{2}, \ldots, x_{m}\right]$ is the features input vector and $m$ is the number of input features.

Encoding categorical values. Many ML algorithms can not directly ingest categorical values. Hence, encoding categorical values is an essential step that must precede the feeding of the data into the predictive model. In this chapter, categorical values are encoded using one-hot encoding.

Dimensionality reduction. User data in wireless networks are characterized by a high number of features, which also means a high number of dimensions. Besides, one-hot encoding will further increase the dimensionality of the data. Highdimensional data are at risk of being very sparse, where most instances are likely to be far away from each other. As a result, predictions using high-dimensional data are much less reliable compared to data with lower dimensions [63]. One way to solve this issue is to increase the amount of training data. However, user data is expensive, difficult to acquire, and not always available to the network. For these reasons, in order to extract relevant behavioral and satisfaction patterns 


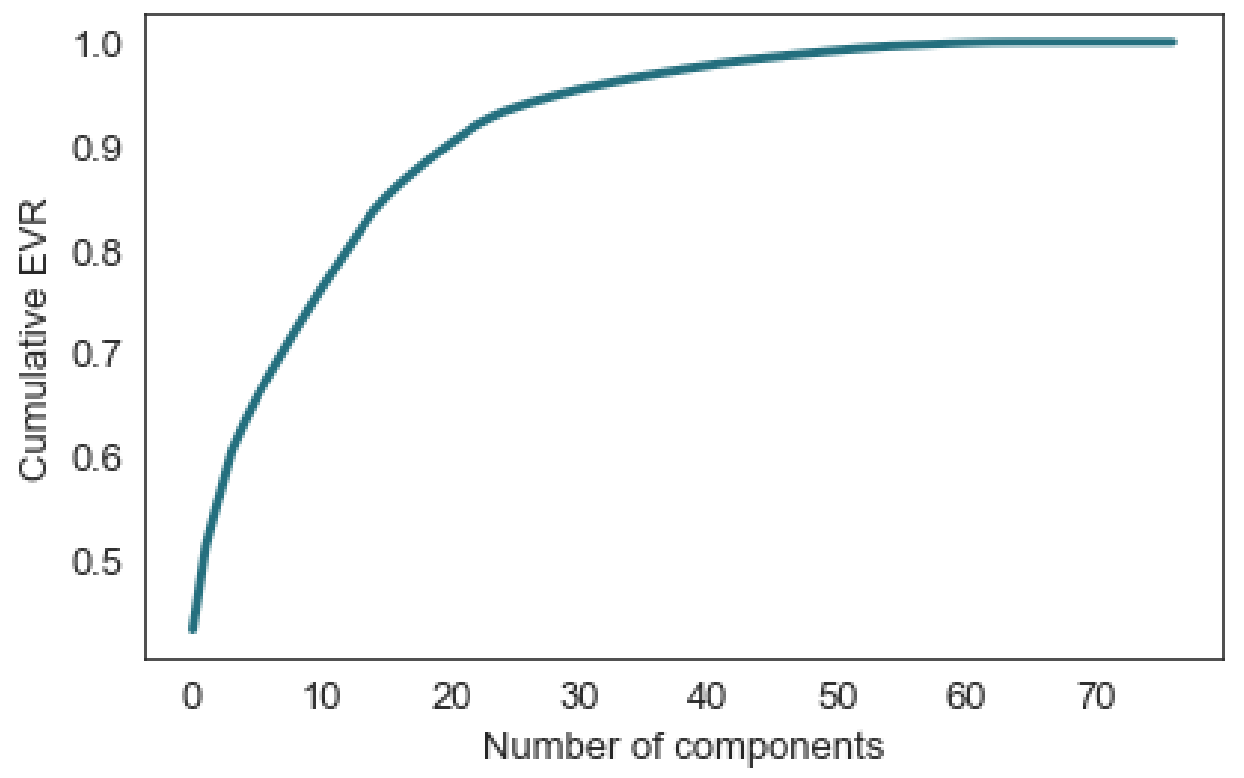

Figure 5.3: Cumulative EVR vs. the number of components.

in the most effective way, the utilization of Principal Component Analysis (PCA) is proposed, which is the most popular dimensionality reduction algorithm [64]. PCA identifies the hyperplane that lies closest to the data and then projects the data onto it. The hyperplane is chosen such that it preserves the highest variance. The PCA algorithm needs the number of principal components to be fed as input. The number of components should be as small as possible while maintaining a reasonable variance in the data. A useful piece of information that can be used to find the optimal number of components is the Explained Variance Ratio (EVR) of each principal component. EVR describes the percentage of the dataset's variance that lies along the axis of each component. Fig. 5.3 illustrates the cumulative EVR as a function of the number of components. For the purpose of this model, it is aimed to preserve at least $98 \%$ variance using the minimum number of components. As shown in Fig. 5.3, 98\% cumulative EVR was achieved using at least 50 principal components.

\subsubsection{Train and validate the model}

There are several ML algorithms that can be used to build a predictive model one of which is the Deep Neural Network (DNN) algorithm. Data collected from wireless networks are colossal and DNNs are fast and more efficient than before in handling huge amounts of data; hence they are extremely attractive for real-time 
applications, such as our proposed framework. It is worth mentioning that the user persona prediction process requires a single ML model to predict all personas.

\subsubsection{Model deployment and production}

Once the output model from the development process has been tested, tuned, and optimized in the development environment, the next step is to deploy the model into production. Production machines are continuously running operating systems with servers that are highly optimized to meet expected load and demand. In this section, the stages of implementing the deployed model into the production process are described.

\subsubsection{Collect data stack for each user}

In this stage, the network collects data from new users joining the network in order to predict their persona type. As mentioned earlier, a user persona describes users with similar user behavior and satisfaction patterns over time. Therefore, in order for the predictor to achieve accurate results, it should be able to decide based on more than one data record. For this reason, the proposed predictor is designed to ingest a stack of data prior to deciding on the predicted user persona. To this end, random statistical sampling without replacement is used in order to build data stacks for different users. Each stack was sampled using the entire population of the test data associated with the ground truth persona. For each persona, 500 stacks of data were sampled for 500 users. Hence, the total number of testing stacks was 2000 stacks with a stack size of $\mathcal{B}$. The effect of changing $\mathcal{B}$ on the performance of the proposed framework will be discussed in Section 5.5.

\subsubsection{Predict user persona for each data record}

For new users, the network collects a stack of data from the user in question and feeds it to the ML model. As shown in Fig. 5.2, each stack is fed into the ML model in order to predict user personas for each data record. At this step, the output of the predictor model is the vector $\mathcal{V}_{i}=\left[v_{i, 1}, v_{i, 2}, \ldots, v_{i, \mathcal{B}}\right]$, where $\mathcal{B}$ is the stack size and $i$ is the user number. Each $\mathcal{V}_{i}$ will be used to predict the final persona label for user $i$. 


\subsubsection{Compute confidence}

After predicting the persona label for each data record, the next stage in the proposed framework is to assess the confidence of the predictor. This step is essential due to the significant impact of using wrong persona labels on the performance of personalized networks. The confidence is computed using two steps. The first step is to compute the histogram of the predicted labels listed in $\mathcal{V}_{i}$ for each user $i$. The output histogram vector is denoted as $\mathcal{H}_{i}=\left[h_{i, 1}, h_{i, 2}, \ldots, h_{i, \mathcal{N}}\right]$, where $\mathcal{N}$ refers to the number of unique persona labels. The second step is to compute the confidence of the prediction by passing $\mathcal{H}_{i}$ through a softmax layer defined by

$$
\mathcal{C}_{i}=\sigma\left(\mathcal{V}_{i}\right)_{j}=\frac{e^{v_{i, j}}}{\sum_{k=1}^{\mathcal{B}} e^{v_{i, k}}}, \quad \forall V_{i, j} \in \mathcal{V}_{i}
$$

The output of the softmax layer is the confidence vector $\mathcal{C}_{i}=\left[c_{i, 1}, c_{i, 2}, \ldots, c_{i, \mathcal{N}}\right]$, where $c_{i, 1}$ is the confidence of persona 1 for user $i$.

\subsubsection{Find the predicted persona}

The next stage is to find the predicted persona label $\hat{P}_{i}$ which has the maximum confidence as follows:

$$
\hat{P}_{i}=\underset{y}{\operatorname{argmax}} \mathcal{C}_{i, y}, \quad \forall y \in \mathcal{Y},
$$

where $\mathcal{Y}=[1,2, \ldots, \mathcal{N}]$.

\subsubsection{Validation}

The proposed persona prediction framework is supported by a validation stage. The validation stage checks whether the confidence of the predicted persona is greater than a specified threshold $\mathcal{C}_{i, t h}$. As shown in Fig. 5.2, if $c_{i, \hat{P}} \geq \mathcal{C}_{i, t h}, \hat{P}$ is passed as the final prediction result. However, if $c_{i, \hat{P}}<\mathcal{C}_{i, t h}$, the network is directed to increase stack size, and hence collect more data from the user. The validated predictions are fed back to the deployed model. The deployed model continuously learns from the arriving samples in a process known as online learning. 


\subsection{Experimental results}

This section assesses the proposed persona prediction framework for personalized wireless networks. As mentioned earlier, the dataset used for our experiment is the UCS dataset, which is publicly available in [39]. In assessing the framework, this section focuses on building, tuning, and optimizing the ML model as well as the implementation of the production process. Besides, a personalized wireless network is built and simulated in order to study the impact of personas on the amount of resources saved as well as the achieved satisfaction levels in personalized networks. The implementation of the experiments was done in Python (version 3.7.6). The DNN model was built using the TensorFlow library (version 2.1). In addition, the scikit-learn library was used for preprocessing the data, whereas seaborn and Matplotlib were used for visualization purposes.

\subsubsection{Experimental settings}

\subsubsection{The ML model design}

In this chapter, the proposed framework is implemented using two different ML algorithms, SVM and DNN. SVM and DNN are well known for their ability to capture complex relations and patterns, and therefore they are great candidates for our experiment. We split the UCS dataset into 70\% training and 30\% testing data. Then, the SVM and DNN models were tuned using grid-search to maximize performance. The tuned SVM parameters were $C=100$ and $\gamma=0.1$. By contrast, the structure of the tuned DNN model was composed of four hidden layers as follows:

- First hidden layer: 1024 neurons.

- Second hidden layer: 1024 neurons.

- Third hidden layer: 512 neurons.

- Fourth hidden layer: 512 neurons.

In addition, we integrated dropout into the DNN model with a rate of 0.15 in order to improve the model generalization and reduce overfitting. 
TABLE 5.3: Cellular network simulation parameters.

\begin{tabular}{ll}
\hline Parameter name & Parameter value \\
\hline Number of available Resource Blocks (RBs) & 12 \\
Number of subcarriers per RB & 12 \\
RB bandwidth & $180 \mathrm{kHz}$ \\
Carrier frequency & $2 \mathrm{GHz}$ \\
UE thermal noise density & $-174 \mathrm{dBm} / \mathrm{Hz}$ \\
Grid Size (k) & 100 \\
Flat fading & Rayleigh \\
Number of personas & 3 \\
Number of users & 3 \\
Number of eNBs & 1 \\
Minimum satisfaction level requirement & 4 \\
Maximum power $\left(P_{\max }\right)$ & 3 Watt \\
\hline
\end{tabular}

\subsubsection{Cellular network environment}

Consider a cell within a cellular network that covers Ottawa, Canada. The cell has one eNB and it is connected to three active users moving within its coverage area. The area of the cell is divided into a $k * k$ grid. The cellular network environment is simulated using the parameters listed in Table 5.3. The cellular network operator collects data about the users and stores it in a database. The collected data are of two types, real-time user satisfaction levels as well as context values, such as time, location, and application. The cellular network operator requires a minimum satisfaction level of 4 for all users. The output $\mathrm{Q}_{\mathrm{S}} \mathrm{p}$ and the satisfaction levels for each user are recorded for 24 hours with a resolution of one second.

\subsubsection{Experiment 1: Performance of ML models vs. stack size}

As shown in the development process (see Fig. 5.2), the ML models were trained using all the labeled data records available in the network. However, in the production process, the prediction was performed using a stack of data for each user. 


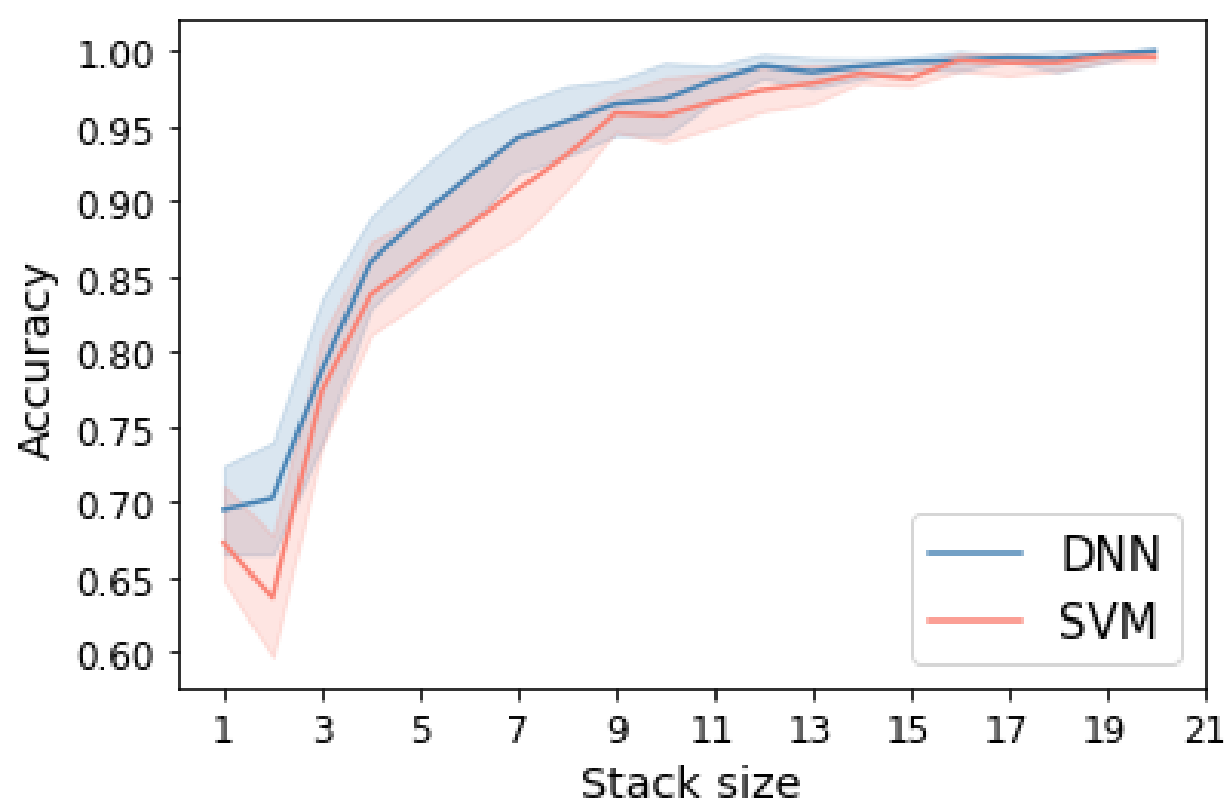

FIgURE 5.4: The accuracy and variance of the proposed persona prediction framework vs. stack size using DNN and SVM.

This enables the ML model to ingest more data before making the final decision about the predicted persona label. One would expect that the more data collected from users, the more accurate the final decision would be. In order to investigate this assumption, we explore the effect of stack size on the performance of our predictors. In Fig. 5.4, we plot the average accuracy vs. stack size for the SVM and DNN predictors, respectively. In addition, we plot the prediction accuracy variance for the four personas using a shaded area around the accuracy curve. As shown in Fig. 5.4, the performance of both predictors improved as stack size increased. Moreover, the variance of the predictors' accuracy decreased significantly as stack size decreased.

Although higher amounts of data improve predictor performance, acquiring data is expensive for the network. Therefore, it is essential to choose an optimal stack size that will reduce the amount of data required without sacrificing a significant portion of accuracy. As shown in Fig. 5.4, the rate of improvement in the predictor's accuracy decreased as stack size increased, almost plateauing at a stack size of 12 . The optimal stack size depends on service provider preferences and the amount and quality of the data available. Service providers demanding higher prediction accuracies should allocate more resources to acquiring more data, which means using a higher stack size. It is also worth noting that the number of persona labels will also affect the performance of the predictors and the required stack size to achieve a certain accuracy. The higher the number of unique persona labels, 


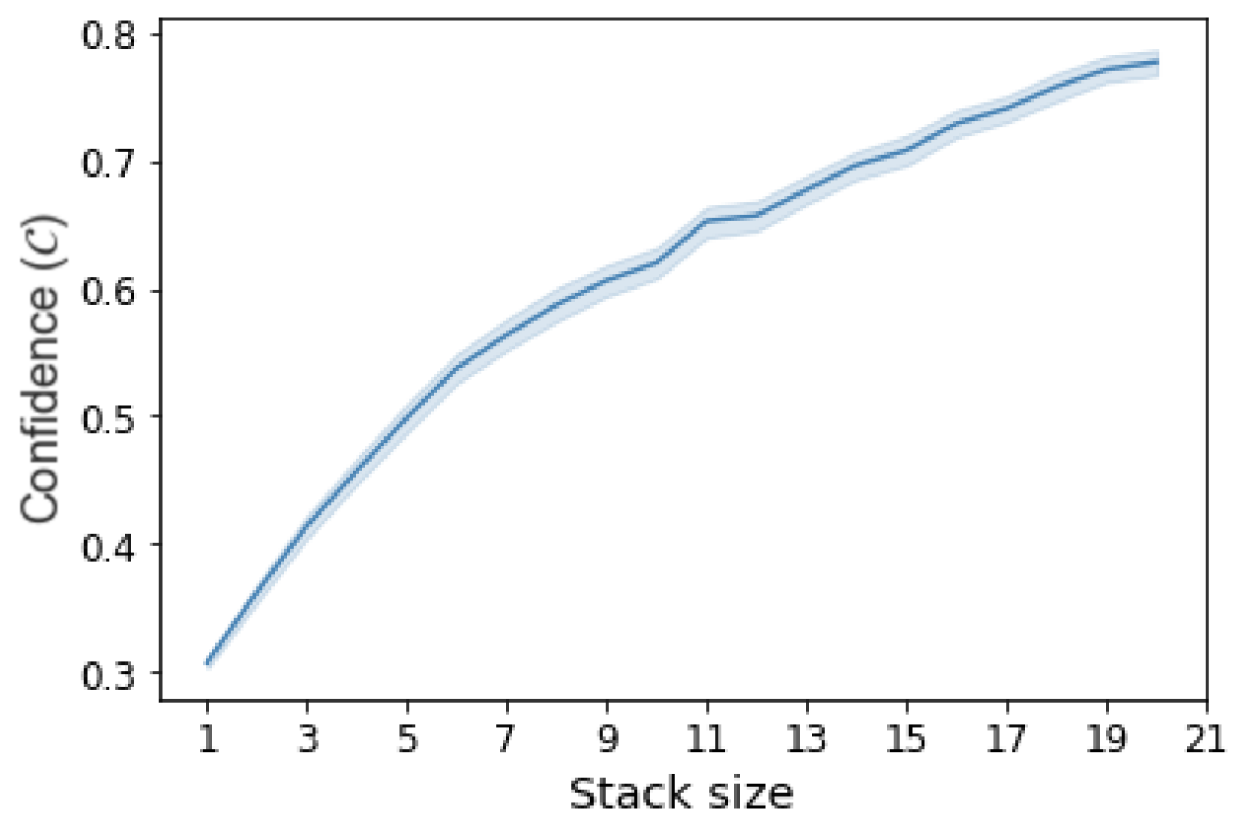

FIGURE 5.5: The confidence and confidence variance of the persona prediction framework vs. stack size using DNN.

the higher the amount of data required to differentiate between users belonging to different personas.

\subsubsection{Experiment 2: Confidence vs. stack size}

As shown in Fig. 5.2, prediction confidence plays a vital role in our proposed framework. Instructing the network to act on the basis of a falsely predicted persona will dramatically affect user experience and satisfaction levels. Since the network has no prior definite knowledge of the persona of new users, the framework is designed to assess the confidence levels of the predictions and consider them only if they pass a certain threshold. In Fig. 5.5, we plot the DNN predictor confidence vs. stack size. It can be seen that, similar to the ML model accuracy, the confidence levels increase with the increase in stack size. Besides, the choice of optimum confidence threshold comes with a tradeoff. The higher the threshold, the greater the amount of data required for the predictions to pass. Interestingly, although the variance of the predictors' accuracy is highly dependent on stack size, the variance in confidence levels for the four personas does not significantly change with the increase in stack size. 


\subsubsection{Experiment 3: Persona in personalized wireless net- works}

As described earlier in this chapter, users associated with the same persona in personalized wireless networks have their unique satisfaction behavior patterns. In other words, they have similar ZoTs; hence similar QoS requirements to achieve a certain satisfaction level. In order to study the behavior of user personas in personalized wireless networks, for the purpose of comparison, we simulate a Personalized (P) and Non-Personalized (NP) network. For the sake of simplicity, we confine the number of users to three users of which each has a distinct user persona. The detected user personas by the simulated personalized wireless network are WP, US, and HM personas. As described in Table 5.3, the minimum satisfaction level required by the service provider is 4 . As a result, based on each persona satisfaction behavior, the personalized network will predict and calculate the minimum amount of network resources, in the form of rate $\left(\mathrm{Q}_{\mathrm{O}} \mathrm{S}_{\mathrm{P}}\right)$, which is required to achieve a minimum satisfaction level of 4 . In comparison to the $\mathrm{P}$ network, the NP network is designed to utilize the available resource such that the maximum rate $\left(\mathrm{Q}_{\mathrm{N}} \mathrm{S}_{\mathrm{NP}}\right)$ is achieved for all users. Fig. 5.6.a illustrates the amount of saved resources by the personalized network (i.e., $\mathrm{Q}_{\mathrm{N}} \mathrm{S}_{\mathrm{NP}}-\mathrm{Q}_{\mathrm{OS}}$ ) for each persona $^{2}$. The amount of saved resources reported in Fig. 5.6. a fluctuate over time. This indicates that the satisfaction behavior of users changes as context changes. As opposed to the NP network, the P network was able to capture these behavioral changes and capitalize on them to save resources. Another important thing to observe is the variance in patterns of saved resources among users, which indicate the existence of different satisfaction behavior for different user personas. For example, the WP persona user showed consistent amounts of saved resources over time, thereby consistent expectations of the network. On the other hand, the HM persona user had lower tolerance during the morning hours; consequently, the amounts of saved resources for this persona was at a minimum compared to other personas. While the personalized network is designed to minimize resources, it is also required to maintain a certain satisfaction level determined by the service provider. Therefore, in Fig. 5.6.b, we study the user satisfaction levels for each persona over the simulated 24 hours. Fig. 5.6.b illustrates a high satisfaction level for all personas in the NP network. This is expected due to the fact that the NP is utilizing more resources compared to the $\mathrm{P}$ network. Nonetheless, while the $\mathrm{P}$

\footnotetext{
${ }^{2}$ For visualization purposes, the output user satisfaction and QoS numbers are averaged every 30 minutes.
} 


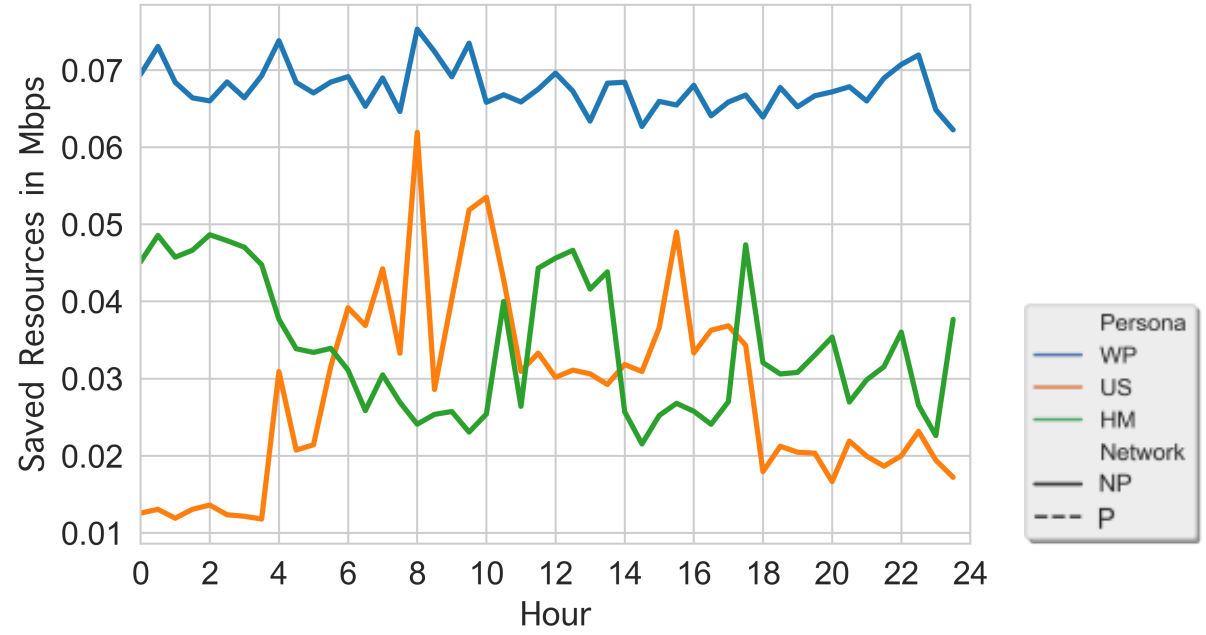

(A) The amount of saved resources by the personalized network in Mbps for each user persona.

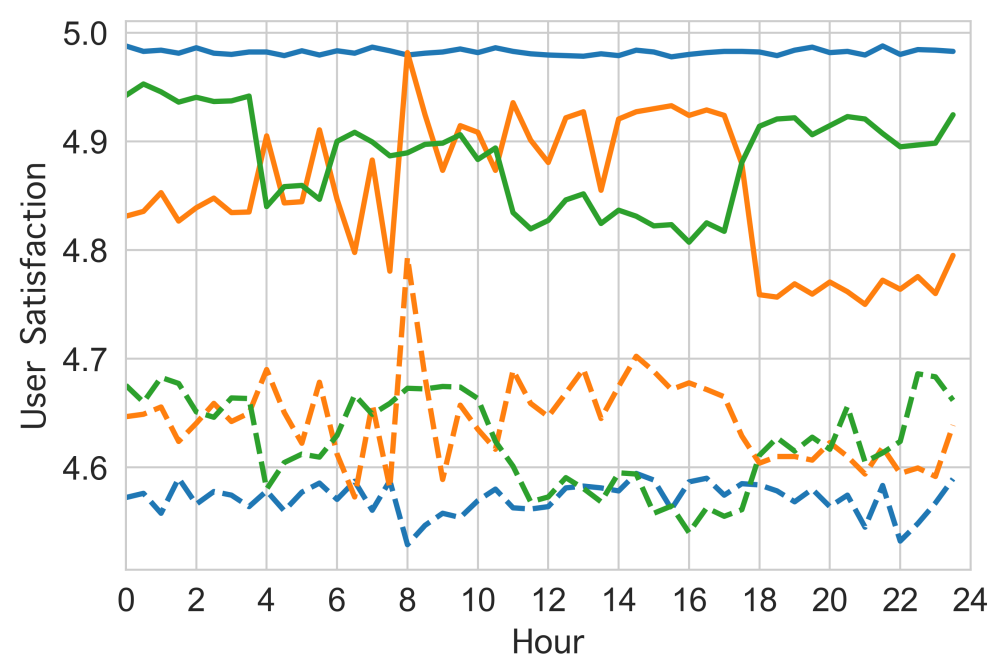

(B) User satisfaction levels achieved by the personalized and non-personalized network for each persona.

FigURE 5.6: Simulation results of the personalized and non-personalized networks. 
network was able to reduce the amounts of consumed resources, it was also able to maintain a satisfaction level above 4 for all personas at all times. It is worth noting that the amount of saved resources in personalized networks depends on several factors including the number of users in the network, the QoS demand associated with the requested service, and the satisfaction behavior of each user captured by the user persona. 


\section{Chapter 6}

\section{User Satisfaction Prediction in Personalized Wireless Networks}

User experience is highly subjective and depends on the highly dynamic user satisfaction behavior in the network. For this reason, data-driven user experience modeling and prediction is more relevant than mathematical modeling. Modeling and predicting user satisfaction in realtime will enable wireless networks to make more personalized decisions, which can increase efficiency and user satisfaction. In this chapter, a framework for building a big data-driven satisfaction monitoring and prediction system is proposed. Inspired by the success of neural networks and deep learning techniques, a deep neural network model to predict user satisfaction in wireless networks using context information is proposed. Also, a comprehensive study is conducted to tune the proposed model in order to achieve the best performance. The results of the experiments show the feasibility and preeminence of the proposed model. 
In wireless networks, user satisfaction is highly subjective, complicated, and changes dynamically depending on various factors. For this reason, mathematical expressions that attempt to model the relationship between user satisfaction and other factors do not yield accurate results. As a result, researchers have begun adopting data-driven approaches that are backed by ML and AI techniques in order to model and predict user satisfaction in wireless networks.

In this chapter, based on the proposed ZoT model in Chapter 3, a data-driven user satisfaction prediction framework is proposed. The proposed framework utilizes context information collected from wireless networks and users to predict in real-time the personalized user satisfaction behavior of each user. Besides, the proposed framework is designed to isolate the process of directly capturing user satisfaction levels from sensor data and the process of real-time user satisfaction prediction using context information. The importance of this property stems from the fact that capturing the actual user satisfaction information directly from users is a cumbersome time-consuming process, hence; it can hinder the proactivity of wireless networks. Instead, predicting user satisfaction behavior based on data flowing in the network is much faster and more efficient. Finally, to illustrate the processes involved in implementing the proposed satisfaction prediction framework, a DNN-based learning model is implemented and tuned.

\subsection{Integrating satisfaction information in wire- less networks: Related work}

The problem of integrating user satisfaction information in wireless networks for the purpose of further optimizing resources has been approached in various ways. The concept of QoE in wireless networks was defined at the International Telecommunication Union (ITU-T) as the "the overall acceptability of an application or service, as perceived subjectively by the end-user." Although this definition is highly subjective, the research community has aimed to narrow it down in order to enable the utilization of such information in wireless networks. In the literature, QoE models are categorized into mathematical models and ML models. Mathematical QoE models are formulations designed to find a specific QoE value for a certain set of parameters. For example, the authors in [65] argue that the QoE has a logarithmic nature described by the law of Weber-Fechner. Experiments and research in QoE modeling have shown that the correlation between variables 
affecting QoE is rather complex and highly dynamic. As a result, mathematical modeling is incapable of accurately modeling or predicting user experience in wireless networks. Therefore, ML has been widely used to capture these complex correlations with a view to providing us with deeper insight and stronger conclusions.

In the literature, the proposed solutions for modeling and predicting user experience in wireless networks differ in terms of input feature design, level of personalization, and ML tools utilized. Researchers have applied different types of ML algorithms, such as Recurrent Neural Networks (RNN), Bayesian modeling, and DNNs [66, 67]. Also, researchers have proposed various input feature designs to feed into their proposed learning models. These designs are categorized as follows:

- Input features are a set of network KPIs [68].

- Input features are a combination of network parameters and measurements aggregated directly from sensors. For example, the authors in [69] proposed monitoring user QoE using a set of network parameters and EEG signals collected from sensors placed near the brain.

- Input features are a combination of context variables and network parameters [70].

- Input features are a combination of context variables, network parameters, and sensor measurements [66].

Lastly, the proposed user experience prediction approaches vary in terms of the level of personalization, which are categorized in two ways: averaged QoE prediction and personalized QoE prediction. Systems based on averaged QoE prediction are designed to perform actions based on the predicted average QoE for all users in the network [68]. Although this approach is simple and easy to implement, it lacks accuracy since user preferences and behavior in wireless networks vary widely depending on complex and dynamic factors. By contrast, personalized QoE prediction aims to predict QoE for each user using data with a set of features recorded from the same user to ensure consistency and relevance [69].

The proposed framework differs from the aforementioned literature work in two aspects. First, the personalized satisfaction prediction solutions in the literature are designed to measure user satisfaction directly from users using sensor data, such as cameras and EEG signals, which is a time-consuming, complex, and cumbersome process. By contrast, the proposed framework in this chapter capitalizes 
on the patterns and correlations that exist between context data and user satisfaction behavior in order to enable real-time satisfaction prediction and tracking in wireless networks. Also, the proposed satisfaction prediction solutions in the literature are based on average-based, static, and highly subjective satisfaction models, such as the QoE model. On the other hand, the proposed framework is based on the proposed ZoT user satisfaction model, which is designed to be dynamic and personalized to each user.

\subsection{User Satisfaction Prediction Framework}

Data collected from communication networks are massive, complex, unstructured, and increase in three dimensions: volume, velocity, and veracity. The problem of extracting user satisfaction knowledge from this huge amount of data presents two subproblems: a big data problem and an AI problem. In this chapter, a novel approach for measuring and predicting user satisfaction in wireless networks is proposed. Fig. 6.2 illustrates the complete framework envisioned for predicting user satisfaction in wireless networks. This framework is designed to autonomously predict future personalized user satisfaction values in real-time for each user in the network in a non-intrusive manner. The proposed framework consists of the following four processes:

\subsubsection{Acquire context and KPI data (Block 1 in Fig. 6.2)}

\subsubsection{Data collection}

The first step is to acquire context data, which can be done by monitoring sensors, aggregating and analyzing collected data, and predicting missing and future context information. Besides, KPI data is collected from the network and aggregated with context information. The authors in [71] provide some details on the process of acquiring and monitoring user context data. As mentioned earlier, using all types of network parameters in the user satisfaction prediction problem is very popular in the literature. However, since user experience is directly influenced by the network KPIs, and due to the fact that, as far as the user is concerned, the changes in network parameters directly affect KPIs, the proposed framework suggests considering KPIs as the only network variables. 


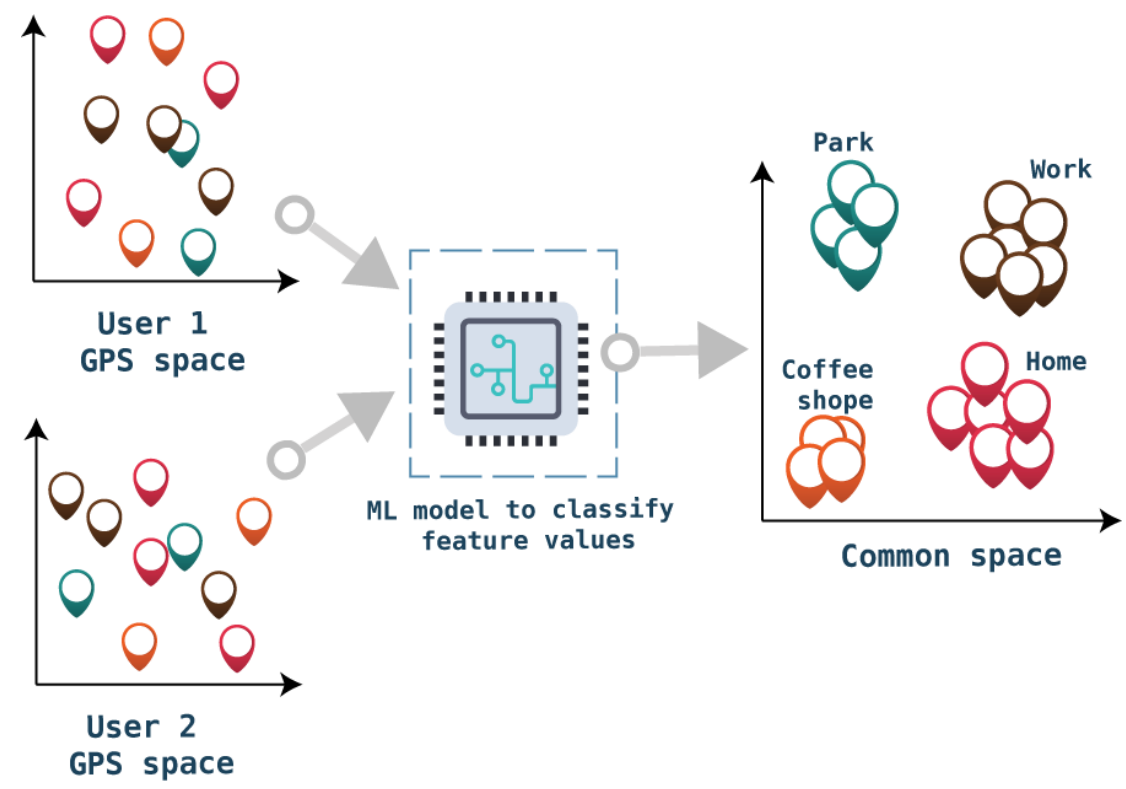

Figure 6.1: An example of data mapping for user location feature.

\subsubsection{Data mapping}

The second step is to map data from different users to shared space. Mapping user data is an essential step as it enables ML models to capture correlations and inherent patterns. For instance, Fig. 6.1 illustrates an example of location feature mapping to a common space. User location is acquired from GPS sensors and its recorded as unique coordinates. Generally, user satisfaction behavior is actually correlated to a particular type of location (e.g., home) rather than GPS coordinates. In order to enable the ML model to extract relevant user satisfaction information, the recorded coordinates should be classified into locations types. In Fig. 6.1, on the left side, feature values (GPS coordinates) are shown in their original input space where each user has his/her own space. After passing the coordinates through the ML model, the common feature space is obtained. In this example, samples belong to four location classes. The color indicates the class of an instance.

\subsubsection{Data preprocessing}

The third step is to retransform data and extract useful features. This step is important because it contributes to the reduction of noise and irrelevant data, which can degrade the predictive model's performance. 


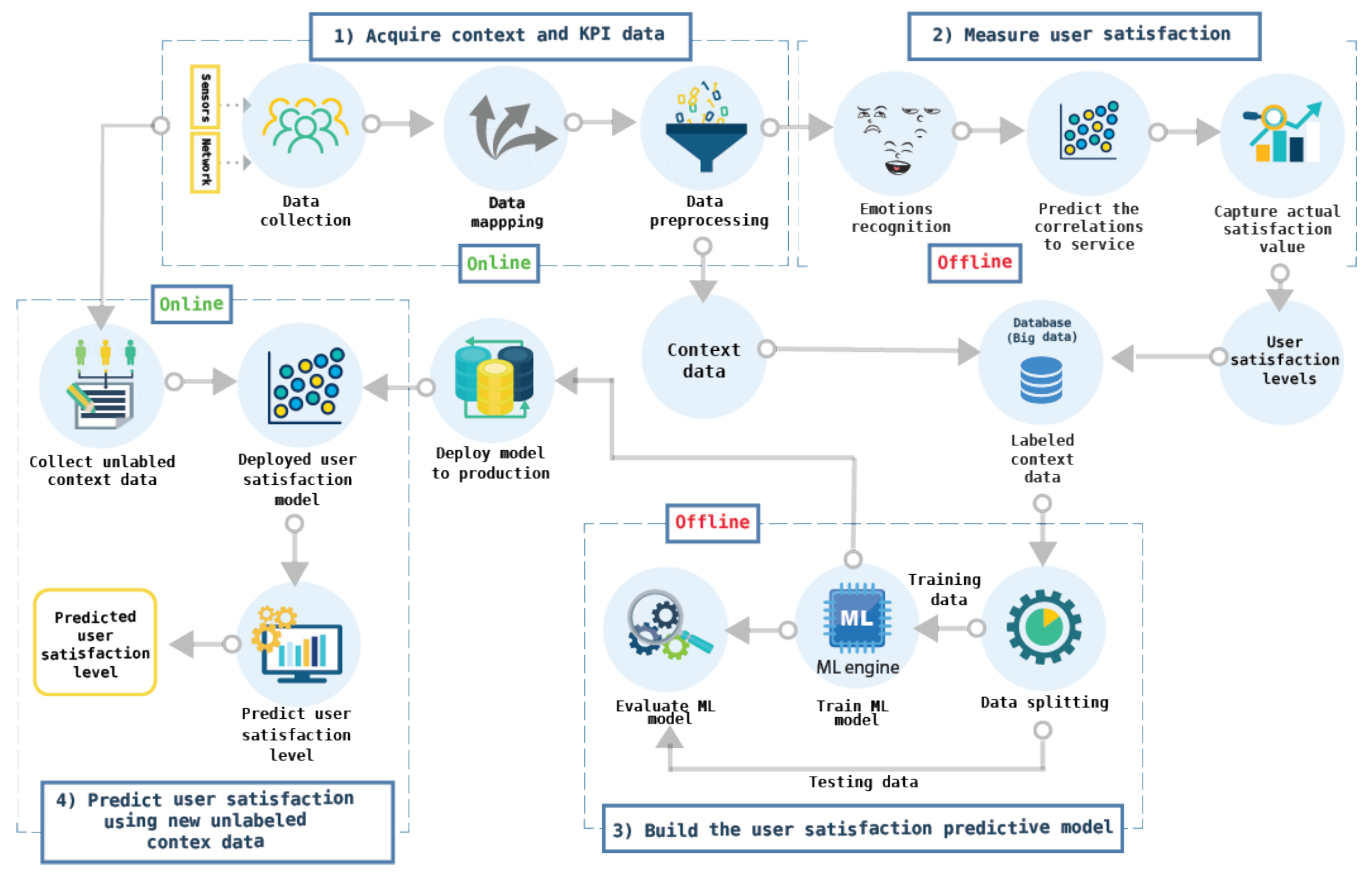

FIGURE 6.2: The proposed big data-driven satisfaction prediction framework. 


\subsubsection{Capture user satisfaction (Block 2 in Fig. 6.2)}

The second process in the framework is labeling context data by the actual user satisfaction values. The proposed process to capture actual user satisfaction is as follows:

\subsubsection{Emotions recognition}

The first step is to predict and measure user emotions and feelings. The detection of user emotions in a non-intrusive manner is widely discussed in computational intelligence literature. Emotions can be monitored using different types of data input, such as images and video [72], speech and sound [73, 74], body language [75-77], and other commercial sensors [78, 79].

\subsubsection{Predict the correlation to service}

The next step is to estimate the correlation between measured user feelings and the performance of the service being used by the user. This step is essential due to the fact that detected feelings are mostly not related to provided service performance.

\subsubsection{Predict the actual user satisfaction}

The next step is to utilize user feelings that are related to the service in order to estimate actual satisfaction levels. Then, the estimated satisfaction levels are used to label context values recorded by the network. Processes 1 and 2 should be designed to collect and label user data in an automated way in order to make the framework scalable and increase network intelligence and the ability to detect users' actual needs and demands.

\subsubsection{Build the predictive model (Block 3 in Fig. 6.2)}

At this stage, the network has enough labeled data to build a user satisfaction prediction model for each user (or persona) with a good performance (predict satisfaction using context information). There are several ML algorithms that can be employed and different techniques to tune the models depending on the type of data being utilized. 


\subsubsection{Predict user satisfaction using new unlabeled data (Block 4 in Fig. 6.2)}

Finally, the fourth process in the proposed framework is to predict future unlabeled user satisfaction values for each user from the context-satisfaction datasets aggregated by the network and stored in the database. In this chapter, deep learning is selected, which is one of the most popular ML tools used to solve various problems in wireless networks [80]. Neural Networks (NNs) that have a sufficient number of hidden layers are an example of deep learning models. There are major advantages to applying DNNs to model and predict user satisfaction levels in wireless networks. Unlike traditional ML tools, DNNs automate feature extraction from data that has complex structures and correlations, which thus reduce expensive human-dependent tasks that hinder automation and real-time network operations. In addition, data collected from wireless networks is increasingly large and heterogeneous and arrives in different formats and speeds from different sources [81]. In contrast to DNNs, the performance of classical ML does not significantly improve with more data and it cannot handle high dimensional data. Thus, DNNs are considered to be one of the best tools for learning useful patterns for complex and colossal wireless network data.

In order to assess the practicality of the proposed framework, the steps that need to be performed during the communication session (i.e., online) should be assessed. As shown in Fig. 6.2, the first process involves relatively fast operations, such as data collection and preprocessing. On the other hand, the second and third processes involve cumbersome, time-consuming, and complex operations, such as training, validating, and implementing the ML models. Nonetheless, since they are implemented offline, they should not affect the network pro-activity. Finally, the fourth process is operating during the communication session and it involves fast operations, such as using the ML models to performance predictions. Therefore, implementing this process in real-time would not raise practicality concerns.

The rest of the chapter discusses further details of the proposed framework by building a DNN model to predict user satisfaction from context data, which are the third and fourth processes in the proposed framework.

\subsection{Dataset Analysis and Prepocesssing}

In this chapter, the working professional persona dataset (WPP) proposed in Chapter 4 is utilized. Table 6.1 shows the features of the WPP dataset and an 
example of their values ${ }^{1}$.

\subsubsection{Dataset filtering and spliting}

The WPP dataset was recorded for a period of one year with one record for each second. However, naturally, the user did not use the network during all measuring instances. Hence, the number of records that actually have satisfaction labels is less than the total number of records. The dataset has an indicator feature called Request arrived which is set to 1 when the user requests a service from the network. The filtered number of records is 100,000. As for splitting, the dataset is divided into $70 \%$ training set, $25 \%$ validation set, and $5 \%$ test set. Stratified

\footnotetext{
${ }^{1}$ The dataset in [39] has other features, such as real sensor measurements. However, in this chapter, only the features listed in Table 6.1 are considered.
}

TABLE 6.1: Features of the WPP dataset.

\begin{tabular}{|c|c|c|c|}
\hline \hline No. & Feature name & Units & Example \\
\hline \hline 0 & Date & - & $2018-01-10$ \\
\hline 1 & Time & - & $14: 55: 02$ \\
\hline 2 & Day & - & Wednesday \\
\hline 3 & Classified days & - & Weekday \\
\hline 4 & Time period & - & Afternoon \\
\hline 5 & Location & - & {$[22,73]$} \\
\hline 6 & Location name & - & work \\
\hline 7 & Speed & $\mathrm{km} / \mathrm{hr}$ & 5.2 \\
\hline 8 & Speed range & - & low \\
\hline 9 & Activity & - & Walking \\
\hline 10 & Request arrived & - & 1 \\
\hline 11 & Application & - & WhatsApp \\
\hline 12 & Service & - & Picture \\
\hline 13 & Demand rate & $\mathrm{kbps}$ & 867 \\
\hline 14 & Min rate & $\mathrm{kbps}$ & 600 \\
\hline 15 & Given rate & $\mathrm{kbps}$ & 802 \\
\hline 16 & $\Delta$ & - & 65 \\
\hline 17 & Max $\Delta$ & - & 267 \\
\hline 18 & Satisfaction & - & 4.0 \\
\hline
\end{tabular}


sampling is used to ensure that the statistics of satisfaction labels are similar for both the training and testing sets.

\subsubsection{Data shuffling and scaling}

In order to improve the DNN model quality and its predictive performance, the features and the satisfaction labels are synchronously shuffled [82]. Also, since feature scaling is a critical step in using DNN models, MinMax scaling is applied, which is typically done via the following equation:

$$
\mathcal{X}_{\text {scaled }}=\frac{x_{i}-\min (\mathcal{X})}{\max (\mathcal{X})-\min (\mathcal{X})}, \forall x_{i} \in \mathcal{X},
$$

where $\mathcal{X}=\left[x_{1}, x_{2}, \ldots, x_{m}\right]$ is the input vector.

\subsubsection{Encoding categorical values}

Neural networks do not accept categorical values. Hence, encoding categorical values is an essential step prior to feeding the data into the model. In this chapter, categorical values are encoded using one-hot encoding.

\subsubsection{Data balancing}

One of the most important preprocessing steps for NNs is to check is the training data is unbalanced. Training NNs using unbalanced data will result in ignoring the classes with smaller representation in the dataset, which creates a biased predictor. Fig. 6.3 plots the histogram for the user satisfaction classes of the considered WPP dataset. The histogram of the classes shows that this dataset is highly imbalanced. In order to address this issue, the dataset is oversampled using the SMOTE algorithm [83].

\subsection{Model Structure and Formulation}

As shown in Fig. 6.4, the network architecture consists of 6 layers feed-forward $\mathrm{NN}$. The first layer is the input data layer. The input matrix is denoted by $\mathcal{X}=\left[x_{1}, x_{2}, \ldots, x_{m}\right]$, where $m$ is the number of inputs (or features) fed into the network. Layers 2 to 5 are the hidden layers, and layer 6 is the output layer with 6 nodes. The number of nodes per hidden layer will be tunned in the following 


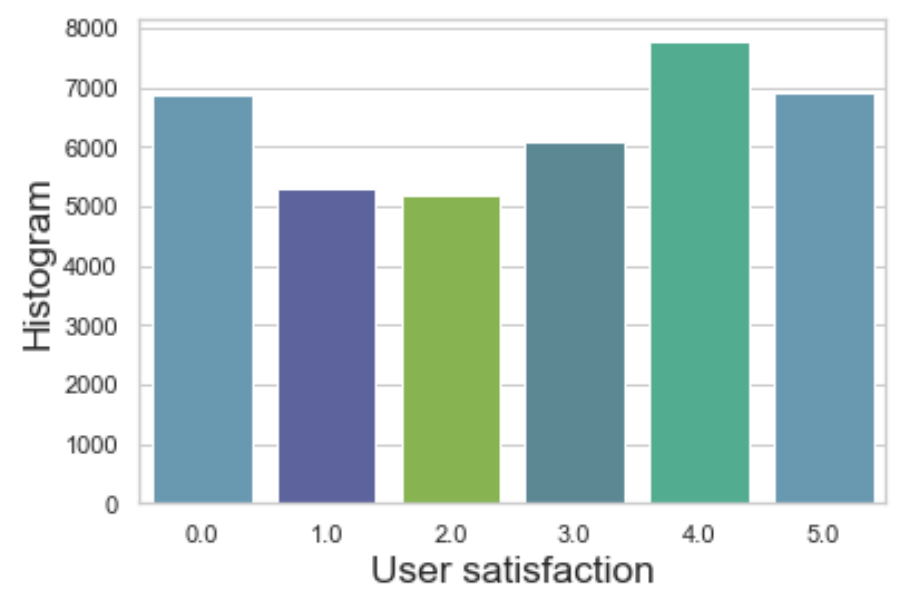

FiguRE 6.3: Histogram for user satisfaction classes of the WPP dataset.

section for the purpose of improving the prediction performance (i.e., classification accuracy) of the model. The output of each neuron is computed through two steps. The first step is to calculate the weighted linear combination of the inputs. Then, the second step is to calculate the nonlinear transformation of the output of the first step. Updating the DNN parameters is divided into two processes:

\subsubsection{Forward propagation}

The output of each layer is updated using the following equation:

$$
v_{i}^{l}=\phi\left(\sum_{\mathrm{i}=1}^{\mathrm{h}_{1-1}} \mathrm{w}_{\mathrm{k}, \mathrm{i}}^{1} \mathrm{v}_{\mathrm{i}}^{1-1}+\mathrm{b}_{\mathrm{k}}^{\mathrm{l}}\right),
$$

where $l$ is the layer, $w_{k, i}^{l}$ is the weight of the connection from neuron $(i, l-1)$ to neuron $(k, l), b_{k}^{l}$ is the bias of the $(k, l)$ unit, and $\phi()$ is a nonlinear activation function. There are several activation functions discussed in the literature. Of these, the rectified linear unit (ReLU) function is selected, which is given by the following:

$$
\phi()=\operatorname{relu}(\mathrm{v})=\max (0, \mathrm{v}) .
$$

The main advantage of ReLU over other activation functions is that it does not activate all neurons at the same time; hence, the neuron will be deactivated if the output is less than zero.

The output score is computed from the first layer to the sixth layer. Since the classification problem is a multi-class problem, the categorical cross-entropy is 


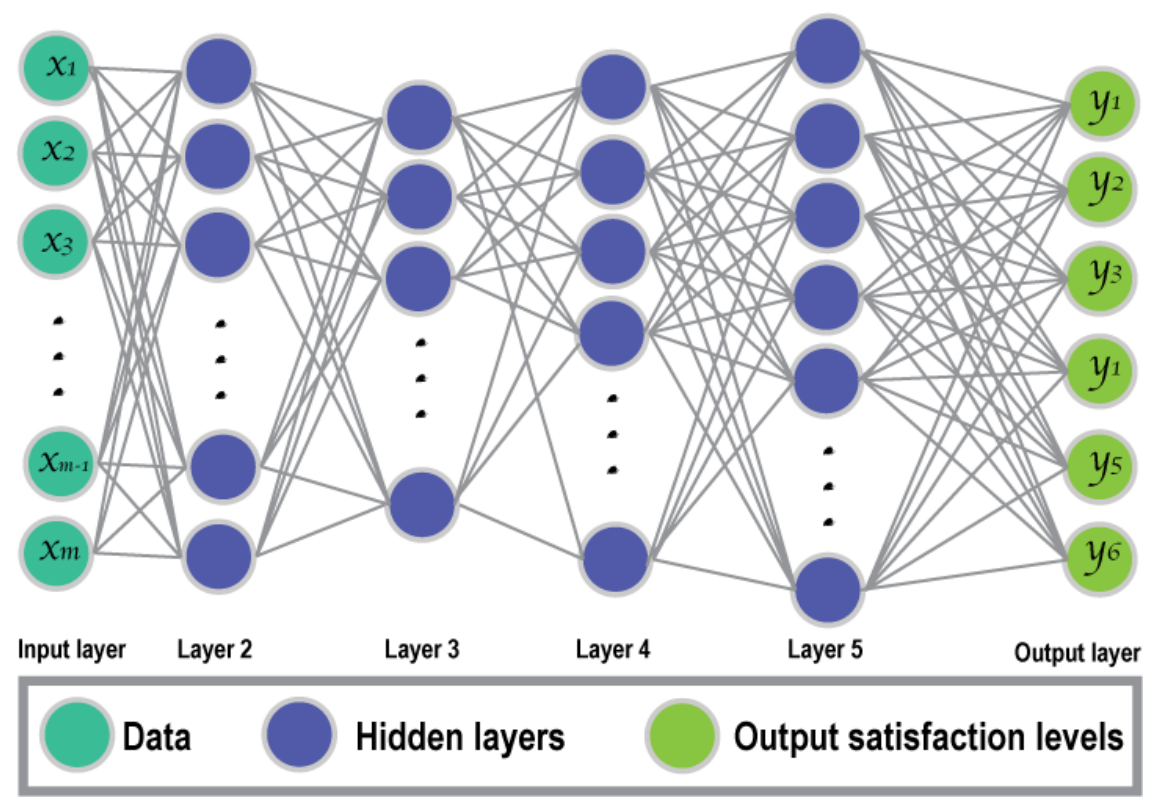

Figure 6.4: The network structure of the proposed DNN model.

used as the cost function, which is given by

$$
\begin{aligned}
& \mathcal{J}(w, b)=\frac{1}{m} \sum_{i=1}^{m} \mathcal{L}\left(\hat{y}_{i}, y_{i}\right) \\
& =-\frac{1}{m} \sum_{i=1}^{m}\left(y_{i} \log \left(\mathrm{v}_{\mathrm{i}}^{|\mathrm{L}|}\right)+\left(1-\mathrm{y}^{\mathrm{y}_{\mathrm{i}}}\right) \log \left(1-\mathrm{v}_{\mathrm{i}}^{|\mathrm{L}|}\right)\right),
\end{aligned}
$$

where $\mathcal{L}$ denotes the loss function, $y_{i}$ and $\hat{y}_{i}$ are the labeled and the predicted outputs, and $L$ is the number of layers.

\subsubsection{Backwards propagation}

After completing the forward propagation calculations, through the hidden layers, the information flows back from layer $L$ to the first layer in order to compute the gradient of $\mathcal{L}$. The parameters of the networks are updated until the loss is minimized. Using gradient descent (GD), the gradient is updated as follows:

$$
w_{t+1, i}=w_{t, i}-\eta \frac{\partial \mathcal{L}}{\partial w} .
$$




\subsection{Experiment Results and Analysis}

The implementation of the experiments in this chapter was done in Python 3.7.6. The DNN model was built using the TensorFlow library. In addition, the scikitlearn library was used for preprocessing the data, whereas seaborn and Matplotlib were used for visualization purposes.

\subsubsection{Improving the performance of DNN}

In order to optimize the performance of the DNN model, various state-of-the-art search and tuning techniques are utilized to yield the best performance.

\subsubsection{Number of layers and hidden units}

The first step is to choose the best DNN architecture that yields the best performance. Hence, a grid search is performed to choose the number of neurons per hidden layer. Based on the output of the search process, the following structure picked:

- First hidden layer (Layer 2): 128 neurons.

- Second hidden layer (Layer 3): 32 neurons.

- Third hidden layer (Layer 4): 16 neurons.

- Fourth hidden layer (Layer 5): 8 neurons.

\subsubsection{Optimization methods}

There are several optimization algorithms proposed in the literature to update the weights of DNNs. The most popular algorithm is GD. For large datasets, GD is very slow because it requires gradient calculation for the entire dataset. In order to rectify this problem, stochastic gradient descent (SGD) is proposed. SGD is faster because it is designed to perform parameter updates for each training example. SGD updates the model parameters according to the following rule:

$$
w_{t+1, i} \Longleftarrow w_{t, i}-\eta \frac{\partial \mathcal{L}\left(w^{T} x_{i}+b, y_{i}\right)}{\partial w_{t, i}} .
$$

Although SGD has improved performance, GD and its variants suffer from several problems including the difficulty of choosing the learning rate and the fact 
that the learning rate is applied to all parameter updates. If the data is sparse, where features have different frequencies, updating all of them is highly inefficient. Adagrad is proposed to allow the learning rate $\eta$ to adapt based on the frequency of the parameter, and therefore, it is well-suited for working with sparse datasets [84]. Adagrad updates the model parameters according to the following rule:

$$
w_{t+1, i} \Longleftarrow w_{t, i}-\eta \frac{\partial \mathcal{L}\left(w^{T} x_{i}+b, y_{i}\right)}{\partial w_{t, i}} \cdot \frac{1}{\sqrt{G_{t}+\epsilon}},
$$

where $G_{t, i i}$ is a diagonal matrix. The main disadvantage of Adagrad is that $\eta$ is always decaying which makes the process slower. Another modified and improved optimizer is the adaptive moment estimation (Adam). Similar to Adagrad, Adam also calculates the adaptive $\eta$ for each parameter. However, unlike Adagrad, Adam also keeps an exponentially decaying average of past gradients [85]. Adam updates the model parameters according to the following rule:

$$
w_{t+1, i} \Longleftarrow w_{t, i}-\eta \frac{\hat{m}_{t}}{\sqrt{\hat{v}_{t}+\epsilon}},
$$

where $\hat{m}_{t}$ and $\hat{v}_{t}$ are the estimates of the first and second moments, respectively.

In order to decide which algorithm best suits the utilized dataset and the DNN model, in Fig. 6.5, the DNN model accuracies for the three aforementioned algorithms are compared. As shown in Fig. 6.5, SGD achieved the lowest accuracy

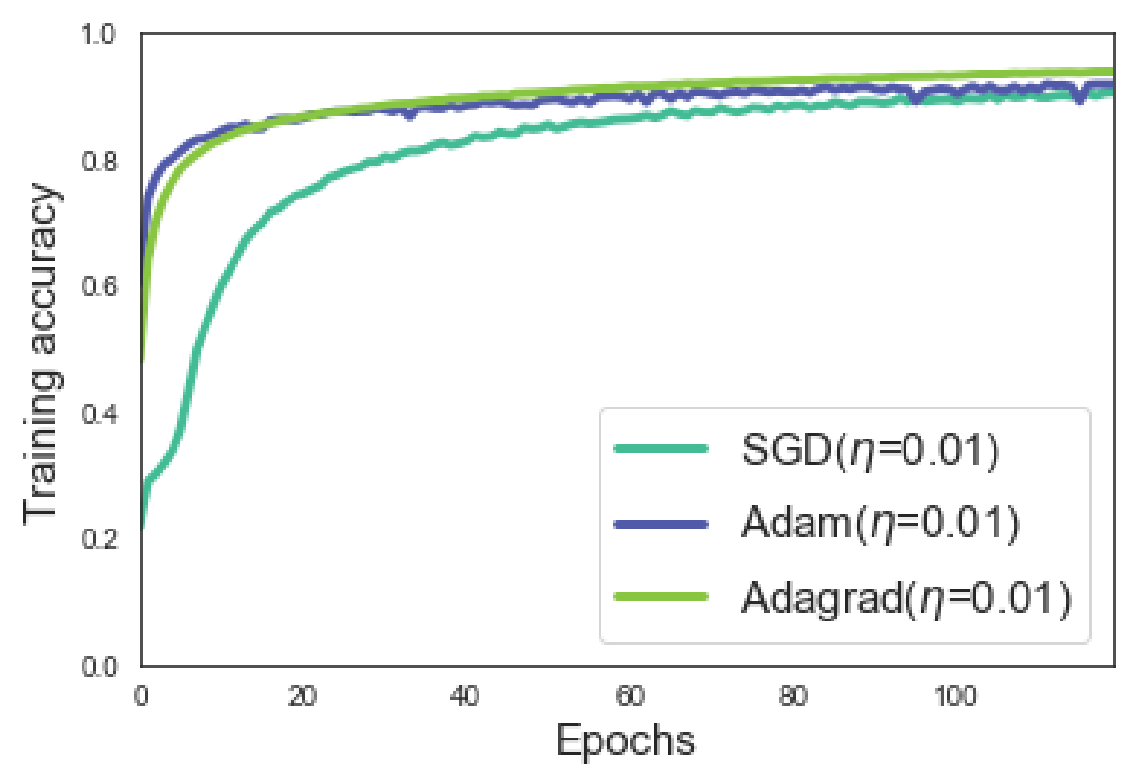

FiguRE 6.5: Comparison between the accuracies of DNNs using different optimization methods. 
convergence. Adagrad achieved the fastest convergence and it converged to the best accuracy, which is why Adagrad algorithm is chosen as for the optimization part.

\subsubsection{Learning rate $\eta$}

Choosing the proper $\eta$ is one of the most important steps in building DNNs. A very small $\eta$ will lead to painfully slow convergence, whereas too large of an $\eta$ could hinder convergence and cause fluctuations. In Fig. 6.6, the training accuracy of the proposed DNN is plotted with the Adagrad optimizer and three different learning rates. The first thing to notice is the very slow convergence of $\eta=0.001$ optimizer. This indicates that $\eta=0.001$ is too small for the DNN model. By contrast, $\eta=0.1$ and $\eta=0.05$ optimizers converge to a low accuracy, which indicates that they are too big for the model. As shown in Fig. 6.6, the fastest and best-performing optimizer is the $\eta=0.01$ optimizer; hence, it is chosen for the proposed model.

\subsubsection{Other tuning parameters}

In addition to the DNN structure, the optimization algorithm, and $\eta$, other parameters are tunned, such as the weight initialization technique. Also, the impact of implementing the following initializers is investigated: zeros, uniform, normal, he normal, and leCun uniform. The results showed that the DNN model achieved the best performance with the leCun uniform initializer. Other parameters, such

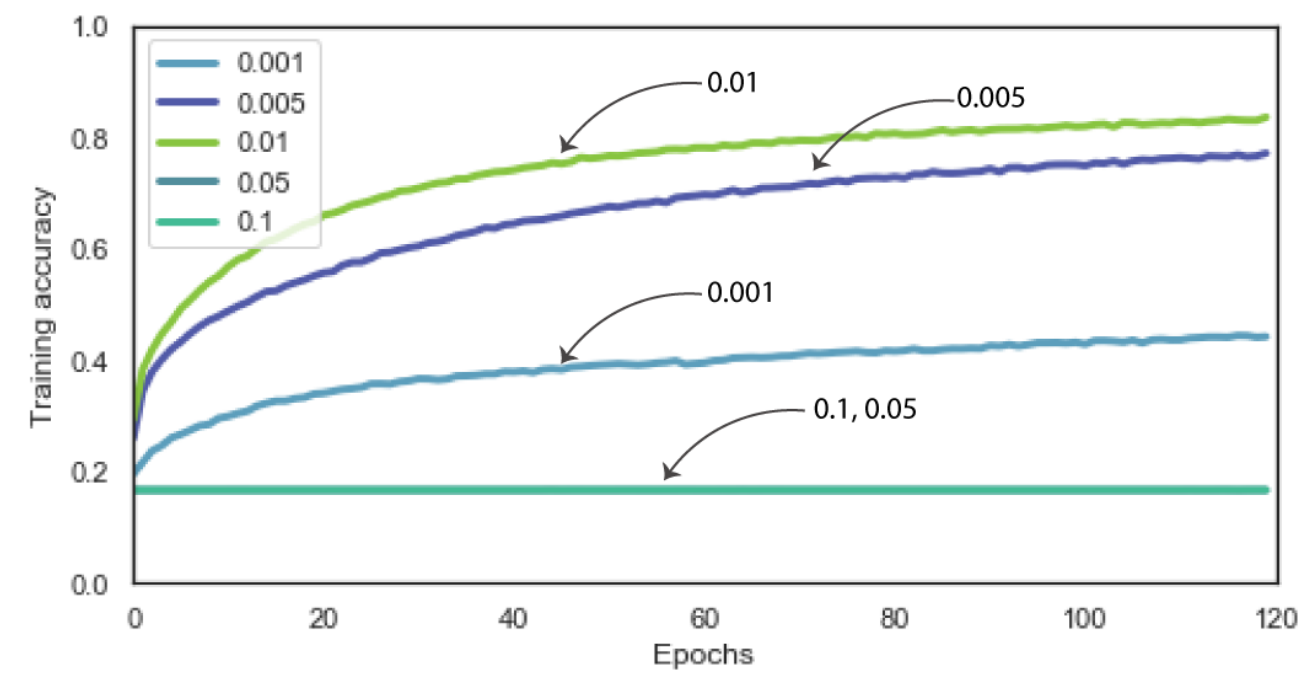

FiguRE 6.6: Comparison between the training accuracy of DNNs using different learning rates. 


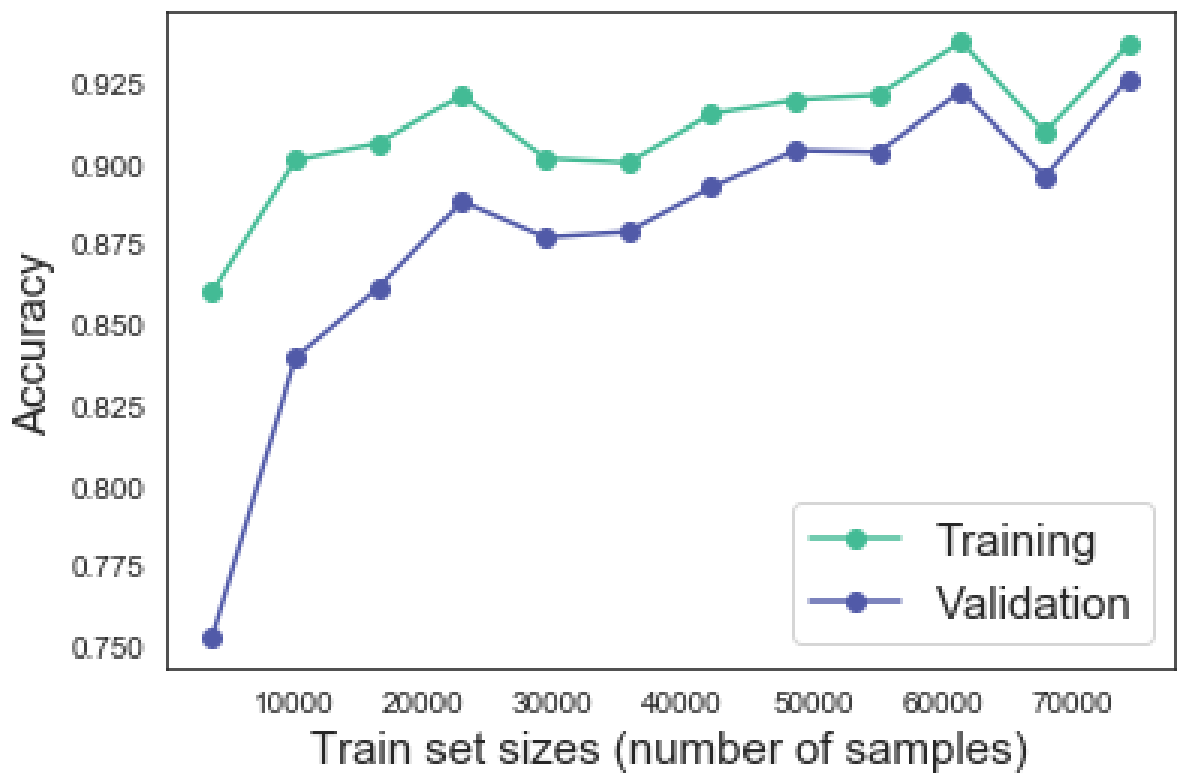

FiguRE 6.7: Comparison of the DNN model performance for different training data sizes.

as batch size and the number of epochs were tuned to 100 and 120, respectively. In addition, regularization is used to reduce overfitting and make the model generalize better. In the literature, there are two main strategies to regularize NNs, dropout and L1 and L2 regularization. L1 and L2 are the most popular types of regularization strategies, and they update the general cost function by integrating another term to reduce the values of the weight matrices. Dropout is also a very interesting regularization technique and it is widely used in the field of deep learning. Dropout randomly picks some nodes and removes them and all the incoming and outgoing connections associated with it. For the utilized DNN design in this chapter, dropout is integrated into each layer. The dropout rate was tuned to $27 \%$ for each layer.

\subsubsection{Performance results}

In order to validate the performance of the DNN model, a CV testis performed. Although CV is not widely used for NNs due to its computational complexity, for the purpose of this study, a 10-fold CV test is performed. For the purpose of comparison, three benchmark ML models are implemented and tuned, namely, Decision Tree (DT), K-nearest neighbor (Knn), and Random Forest (RF). Fig. 6.7 depicts the 10-folds CV accuracies for the four ML models. As shown in Fig. 6.7, the DNN model achieved the best average accuracy ((93.78 \pm 0.51$) \%)$. Also, Fig. 6.7 shows that the DNN model is more robust in comparison to the other 


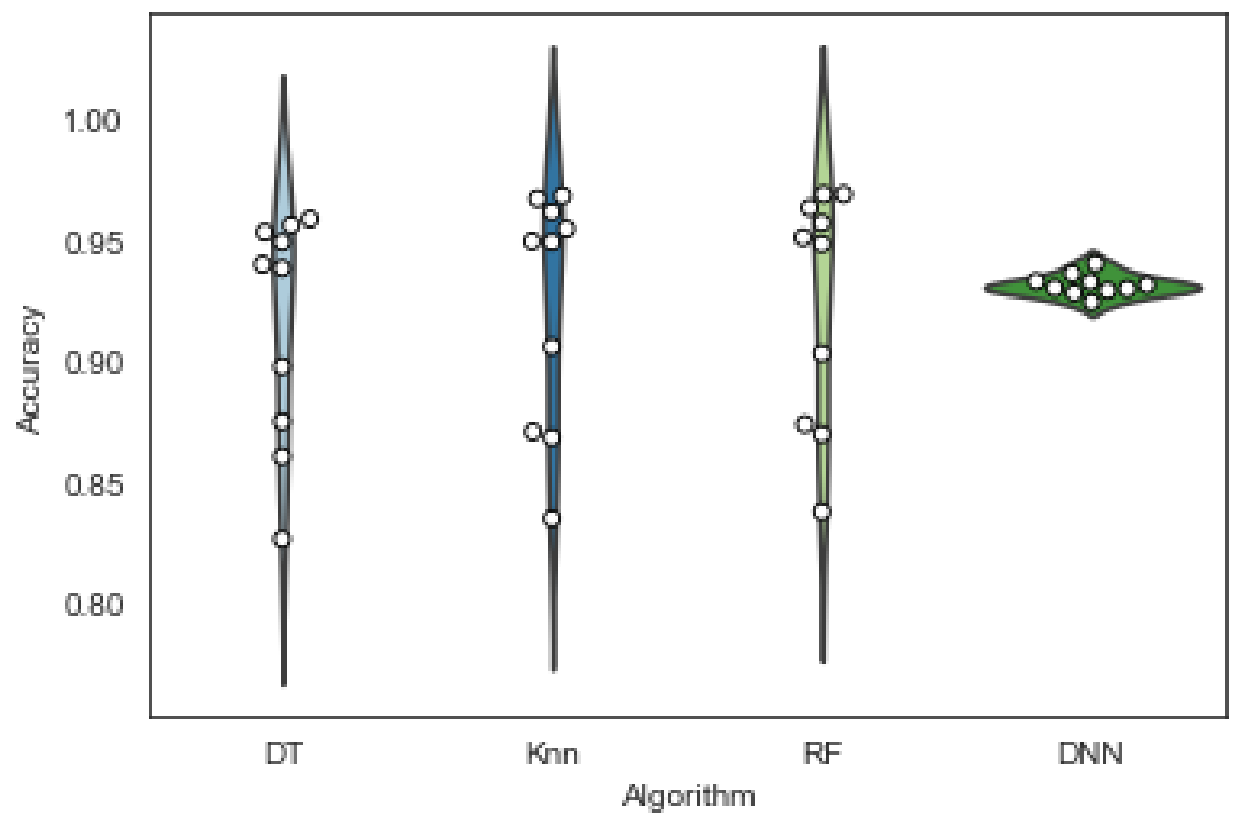

FiguRE 6.8: Accuracy swarm plot for 10-folds cross-validation using DT, Knn, RF, and the DNN model.

ML models as it achieved the lowest confidence interval. It is worth noting that since there are six satisfaction levels, the random choice accuracy level is 0.166. Therefore, an average accuracy of $93.78 \%$ is considered very good and it can be further improved by training with more data. Finally, it is important to point out that the results reported in this section are based on the synthesized dataset designed in Chapter 4; hence, the hyper-parameter estimation and even the model structure would need to be revisited when real data becomes available. 


\section{Chapter 7}

\section{Personalized Resource Allocation in Wireless Networks: An AI-Enabled and BD-Driven Multi-Objective Optimization}

This chapter proposes a Multi-Objective Optimization (MOO) formulation to model the personalized resource allocation problem in wireless networks. The proposed MOO problem is tackled using evolutionary optimization due to its practicality and speed. Also, statistical analysis is conducted to verify the significance of the obtained results. Besides, a simulation proof-of-concept prototype is built to solve the formulated problem. The prototype is utilized to demonstrate the benefits of implementing personalized networks in contrast to non-personalized networks. Further, the effect of uncertainty introduced by the ML surrogate models was examined. Lastly, a scalability analysis was performed to investigate the effect of increasing the number of problem variables, such as the number of users, on the complexity and quality of solutions. 
The main contribution of this chapter revolves around addressing the personalized decision-making process that is responsible for making optimized, fine-grained, and personalized actions in wireless networks. The decision process in personalized wireless networks is based on the intelligence created by ML engines. The primary use of ML in personalized networks is to build surrogate models for user satisfaction behavior, which is highly dynamic and continuously evolving. This chapter begins by presenting some fundamental concepts associated with personalized wireless networks. Then, of the various decision-making processes in wireless networks, this thesis sheds light on the personalization of the resource allocation process. The main premise of personalized resource allocation is to achieve optimum allocation such that maximum user satisfaction levels are achieved using the minimum amount of resources. To this end, personalized wireless networks should be designed to optimize two correlated and contradicting objectives in real-time: user satisfaction and resource utilization. In this thesis, the described optimization problem is termed by the Optimum Personalized Resource Allocation (OPA) problem.

In this chapter, OPA is modeled as a Multi-Objective Optimization (MOO) problem. In wireless networks, decisions are made in real-time; hence, to maintain the proactivity of the network, the optimization process and decisions are also required to be in real-time. As a result, although exact optimization algorithms that are based on mathematical programming produce the best possible solutions, they are slow and computationally expensive; hence not feasible due to the complexity of OPA. Instead, to speed up the optimization process, data-driven evolutionary optimization is utilized to approximate the Pareto front solutions. In view of this, a review and categorization of data-driven Evolutionary MOO (EMOO) are presented followed by a discussion on the benefits and challenges of employing EMOO in personalized wireless networks. Another important aspect of the problem is integrating user satisfaction behavior into the optimization process. To actualize this in real-time, a surrogate model is utilized to approximate the personalized user satisfaction behavior of network users. The proposed surrogate model is ML-based and built using Deep Neural Networks (DNNs). Then, in order to maintain and manage the surrogate models, a surrogate-management framework is proposed. The framework employs the collection of select user satisfaction feedback measurements in real-time. Also, it reduces the risk of solutions divergence and the effect of uncertainty introduced by surrogate models. Besides, it is designed to continuously enhance the performance of the surrogate models as more data arrive in the network. Afterward, the OPA problem is formulated and solved 
Chapter 7. Personalized Resource Allocation in Wireless Networks: An AI-Enabled and BD-Driven Multi-Objective Optimization

using EMOO. Through several experiments, the optimum Pareto front solutions are analyzed for various EMOO algorithms. Then, using the best algorithm, the personalized and non-personalized networks are compared in terms of the amount of saved resources and user satisfaction levels in the network. Moreover, the effect of uncertainty introduced by the surrogate models on the quality of the produced Pareto front solutions is studied. Finally, a scalability analysis is conducted to explore the effect of higher numbers of users and the effect of varying the Number of Function Evaluations (NFEs) on the performance of the simulated algorithms and the quality of solutions.

\subsection{Data-Driven Surrogate-Assisted Evolution- ary Multi-Objective Optimization}

Many problems in all sorts of research fields are formulated as optimization problems. While optimization problems were traditionally approached using mathematical programming, the complexity level of current problems have led researchers in academia and industry to move towards more heuristics/metaheuristics approaches. In contrast to mathematical programming, heuristics/metaheuristics optimization algorithms are less sensitive to the formulation of the optimization problem. This is considerably important for wireless network optimization problems due to their scale and complexity level. Generally, heuristics/metaheuristics optimization algorithms are of two main classes, Evolutionary Algorithms (EAs) [86] and Swarm Intelligence-based Optimization Algorithms (SIOAs) [87]. In this chapter, EAs are employed to solve the proposed and formulated optimization problem.

\subsubsection{Evolutionary optimization algorithms}

EAs are a class of metaheuristics population-based optimization algorithms, where multiple candidate solutions are maintained in parallel. EAs are designed based on the idea of the survival of the "fittest" solution in order to evolve a population that is a good approximation of the global optimum [88]. The fitness of an evolved solution is a measure of its quality at solving the problem. Block 1 in Fig. 7.1 illustrates the process cycle of evolutionary computation. At each cycle, EAs begin with generating parents (populations of candidate solutions). Then, offspring solutions are generated using various variation operations, such as crossover and 


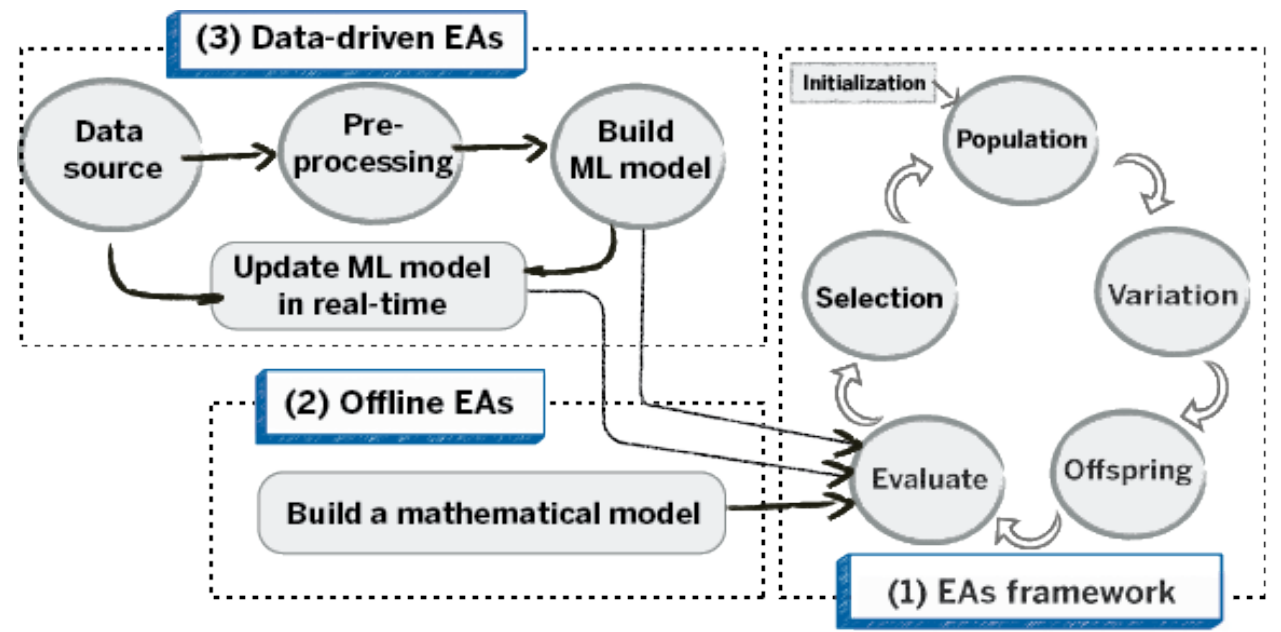

Figure 7.1: A comparison between data-driven (online) and offline EAs frameworks.

mutation. Lastly, in order to select the parent solution for the next cycle, the quality (or fitness) of the generated offspring solutions are evaluated using the objectives and constraints.

There are several advantages of EAs that drive researchers to utilize them for solving various optimization problems, of which the most important is that they do not necessitate analytical modeling and formulation of the objectives and constraints functions associated with the optimization problem. This is considered beneficial since many emerging problems, including the proposed OPA problem, can not be expressed using mathematical modeling. The mathematical formulation could require the integration of many relaxations into the problem, which, in effect, will limit the practicality and accuracy of the produced and solved problems. Another important advantage of EAs is that they can operate with little domain knowledge, which creates more robust models that are less susceptible to the various mathematical features of problems, such as convexities and discontinuities. Another advantage that adds to the robustness of EAs is that they are based on stochastic search, which samples the entire population making them less vulnerable to local optimums.

The advantages of heuristics/metaheuristics optimization come at a price. Since EAs rely on iterating the evaluation process of the objective and constraints associated with the solutions population, the higher the degree of accuracy required, the higher the computational power required. However, supercomputers and distributed computing technologies are improving rapidly to the point that the use of big data analytics and EAs for practical near-real-time applications are currently possible. As a result, EAs have been utilized in many fields and applications, 
Chapter 7. Personalized Resource Allocation in Wireless Networks: An AI-Enabled and BD-Driven Multi-Objective Optimization

such as automated data mining to extract meaningful information [89], finance and economics[90], and wireless sensor networks [91].

\subsubsection{Online vs. offline optimization}

Although EAs do not require the analytical and mathematical formulation of objectives and constraints, most EAs in the literature assume the availability of such models. Optimization algorithms that assume the availability of mathematical models to assess objectives and constraints are referred to as offline optimization algorithms. Unfortunately, offline optimization is not a valid approach for many dynamic problems that involve rapidly changing features, requirements, and behaviors. In comparison, online optimization continuously digests data from the problem's environment to make decisions based on updated information flowing to the algorithm, making the optimization process more dynamic and adaptive. EAs that are based on data are called data-driven evolutionary optimization algorithms. As shown in Fig. 7.1, the main difference between offline EAs (Block 2, Fig 7.1) and data-driven EAs (Block 3, Fig 7.1) is the method used in the evaluation process. Offline EAs utilize analytical objective and constrainest to evaluate the fitness of solutions, whereas data-driven EAs utilize data-driven models. The proposed OPA problem in this chapter utilizes a data-driven model to estimate real-time user satisfaction in networks; hence, data-driven (online) EAs are utilized to find the optimum decisions.

\subsubsection{Interactive evolutionary computation}

Interactive Evolutionary Computation (IEC) is evolutionary computation applied to optimize systems based on humans' subjective opinions and expectations [92]. The IEC technology embeds a user in the optimization system in which the user is considered to be a black box. There are several reasons for integrating user input with particularly EAs as opposed to other optimization methods. One of the most prominent reasons is that EAs do not require the use of gradient information to search the space, which in most scenarios can not be computed for such problems. Examples of problems that capitalize on IEC algorithms include mental health measurement [93] and emotional music generation [94].

Although the decisions in personalized wireless networks are optimized based on users' subjective opinions and expectations, users are not actively logging their satisfaction levels. Instead, as shown in Fig. 7.2, user satisfaction is captured in 
a non-intrusive manner from sensors data using AI. Also, the trained user satisfaction ML engine models user behavior and expectations, which enables the network to repeatedly evaluate user satisfaction in the optimization process in a non-intrusive manner and in a relatively short time. Considering the fact users are involved in the personalized optimization process, the proposed OPA problem in this chapter can be considered as an IEC problem. In the literature, many researchers proposed solutions to improve the performance and efficiency of IEC problems. For example, preference-based (or progressive) interactive evolutionary optimization reduces the required numbers of function evaluations by involving the decision-maker in the intermediate generations of the algorithm; hence focus computations on the targeted Pareto front solutions [95, 96]. Even though IECbased algorithms have several benefits and can greatly enhance the performance of optimization algorithms, this chapter employes well-known Multi-Objective Evolutionary Algorithms (MOEAs) that are not necessarily optimized for IEC problems.

\subsection{Surrogates in Personalized Wireless Networks}

The micromanagement and personalization of wireless networks require the continuous tracking and measurement of personalized user satisfaction behavior for all users. Such a level of granularity and dynamic behavior modeling can not be achieved using the traditional mathematical models or the average-based ML models proposed in the literature [65-67, 97]. Therefore, in Chapter 6, a big datadriven satisfaction prediction framework is proposed, which is designed based on the proposed user ZoT model discussed earlier. The framework composes personalized ML models optimized based on context data collected by the network. The output personalized satisfaction behavior models are employed by personalized networks to personalize the allocation of resources based on the satisfaction behavior of each user.

\subsubsection{Why we need surrogates?}

In the optimization literature, ML models that are used to estimate the relationship between the inputs and outputs of systems are known as ML-based surrogate models (or meta-models). In this section, the main drivers for adopting surrogates models in personalized wireless networks are discussed. 
Chapter 7. Personalized Resource Allocation in Wireless Networks: An AI-Enabled and BD-Driven Multi-Objective Optimization

\subsubsection{Real-time user satisfaction evalulation}

Although user satisfaction behavior in wireless networks can not be modeled analytically, the network can still capture the satisfaction measurements from users. Although these measurements may not be done in real-time, they can act as the exact function evaluations (or reference evaluations) for the evolutionary optimization algorithm. The problem with measuring user satisfaction is the associated cost. Collecting data and extracting user satisfaction information is expensive and time-consuming. Due to the nature of wireless network applications, wireless networks are designed to take decisions in real-time. Therefore, for practical reasons, real-time network decisions should not be designed to be dependent on the captured user satisfaction. Evolutionary optimization of wireless network problems will not be able to afford thousands of real-time evaluations required by EAs. Instead, surrogate models are comparatively faster and rely on historical data and user patterns to predict user satisfaction values in real-time. Accordingly, surrogate models are considered an essential enabler for personalized wireless networks.

\subsubsection{Dynamically evolving user satisfaction models}

Our expectations of wireless networks dynamically change with several factors that constitute multi-variate contexts. Also, the speed of behavioral changes can be in the order of seconds, which imposes another limitation on the modeling process of user behavior in wireless networks. Although users could have repeating patterns in their short term behavior and expectations, their long term behavior and satisfaction patterns can change. This can be explained by different factors, such as age, professional development, career type, etc., which are usually accompanied by interest changes. Since the surrogate models are built using ML models, and due to the fact that such surrogates are continuously trained and validated through user feedback data, they are able to capture the short term changes in user behavior patterns as well the behavioral changes over the long run. This ensures the dynamic design and adaptivity of the network.

\subsubsection{Management of surrogates in personalized networks}

Management of surrogates, which involves the process of using and updating the models, plays an essential role in maintaining acceptable performance of surrogateassisted optimization [98]. Generally, surrogate models are assumed to be of high 


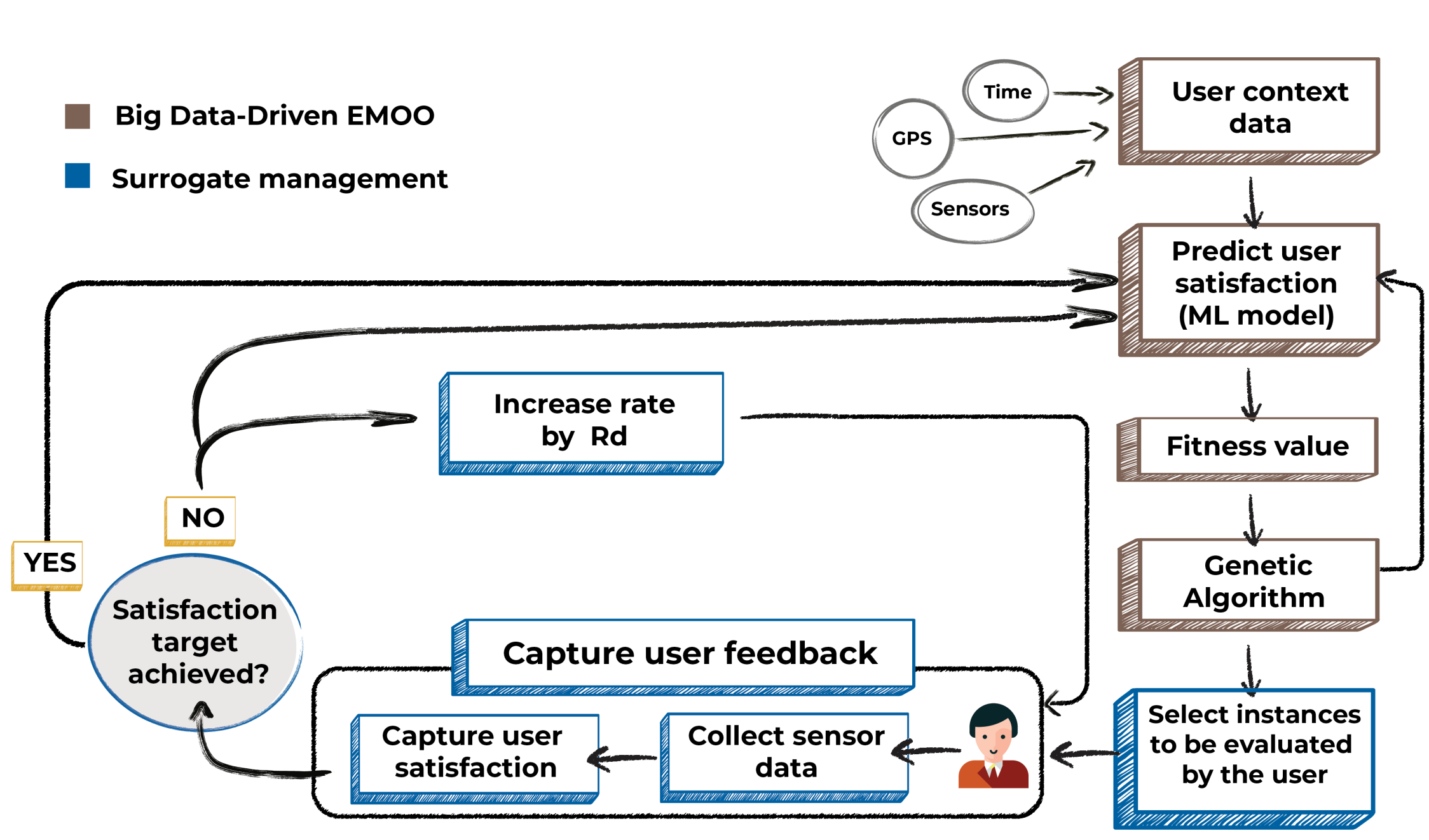

FiguRE 7.2: EMOO management framework to optimize personalized wireless networks. 
fidelity; hence, the exact fitness functions are not utilized in the evolutionary optimization computation. Nevertheless, this approach can run the risk of convergence to incorrect solutions [99].

To address this issue, many strategies for managing surrogate models using exact fitness functions are proposed in the literature [98]. Nonetheless, since the OPA problem proposed in this chapter does not have an exact fitness function, the implemented MOEAs are solely dependent on the approximated ML-based surrogates. Hence, to manage user satisfaction surrogates in personalized wireless networks, a different approach is adopted. Fig. 7.2 illustrates the proposed framework for solving the OPA problem using evolutionary computation. As shown in Fig. 7.2, the proposed framework essentially relies on the trained ML-based user satisfaction surrogates to compute the satisfaction fitness values during the optimization process. However, even though the exact fitness function for user satisfaction behavior in personalized wireless networks does not exist, user satisfaction feedback can be measured and utilized to enhance the accuracy of the implemented surrogates and prevent the network from converging to inaccurate solutions. As shown in Fig. 7.2, user satisfaction feedback is measured and fed back to the surrogate model in order to actively validate and correct inaccurate solutions produced by the optimizer. Also, the surrogate model uses the continuously arriving data samples to learn and enhance its performance. Moreover, when the measured user satisfaction levels do not match the optimized targeted levels by the optimizer, as illustrated in Fig. 7.2, the proposed framework suggests to gradually increase/decrease the provided resources to the user by $R_{d}$ while continuously measuring the actual user satisfaction levels. Then, the collected user satisfaction behavior data at that particular context is used to enhance the performance of the approximated ML-based satisfaction surrogate. This process is critical as it prevents wireless networks from continuously providing services to users with satisfaction levels that do not meet the service providers' standards and requirements.

Notably, only a small number of instances are re-evaluated and validated by the real user satisfaction behavior in the network. There are several algorithms and solutions proposed in the literature for choosing such individuals or instances, including selecting solutions with high uncertainty levels [100, 101], and choosing representative and good solutions [102-104]. The implementation and integration of these strategies into the proposed framework in Fig. 7.2 is out of the scope of this thesis. 
Chapter 7. Personalized Resource Allocation in Wireless Networks: An AI-Enabled and BD-Driven Multi-Objective Optimization

\subsubsection{Surrograte model design and performance}

Various ML models can be utilized to build surrogates, including linear models, support vector machines [105], and Gaussian processes [106]. In Chapter 6, a big data-driven framework is proposed to build ML-based surrogate models in order to predict user satisfaction in wireless networks. The proposed framework is designed to automate the process of data collection, preprocessing, prediction, and validation, which maintains a seamless integration with the highly dynamic design of wireless networks. In this chapter, DNNs are adopted to capture the complicated patterns that exist within the collected user data. Since building accurate and complex ML engines to predict user satisfaction in wireless networks is not the main focus of this chapter, a simple DNN model is adopted with four layers as follows:

- First hidden layer (Layer 2): 128 neurons.

- Second hidden layer (Layer 3): 32 neurons.

- Third hidden layer (Layer 4): 16 neurons.

- Fourth hidden layer (Layer 5): 8 neurons.

The data fed into the model is pre-processed using several steps including scaling, encoding, and balancing. Chapter 6 discusses the details of the implemented preprocessing steps for the personalized networks dataset utilized in this chapter. To examine the performance of the utilized DNN model in this chapter, a 10-folds cross-validation test is implemented. Table 7.1 summarizes the performance of the implemented ML model.

TABLE 7.1: Performance of the adopted DNN design in terms of accuracy.

Accuracy of individual folds in \%

\begin{tabular}{cccccccccc}
\hline \hline fold-1 & fold-2 & fold-3 & fold-4 & fold-5 & fold-6 & fold-7 & fold-8 & fold-9 & fold-10 \\
\hline \hline 95.45 & 95.76 & 94.61 & 95.66 & 95.64 & 94.93 & 95.46 & 95.54 & 95.30 & 95.41 \\
\hline \hline \multicolumn{3}{c}{ Average Accuracy } & \multicolumn{5}{c}{ Std. of Accuracy } \\
\hline \hline & \multicolumn{5}{c}{$0.34 \%$} \\
\hline \hline
\end{tabular}


Chapter 7. Personalized Resource Allocation in Wireless Networks: An AI-Enabled and BD-Driven Multi-Objective Optimization

\subsection{Data-Driven Multi-objective Optimization of Resources in Personalized Wireless Networks}

In order to study the benefits of integrating personalization into wireless networks, the resource allocation problem for personalized wireless cellular networks (i.e., OPA) is formulated and solved in this chapter. As shown in Fig. 7.3, the resource allocation algorithm for personalized wireless networks accepts two groups of inputs, user context values, and network/system context values. User context is a set of variables that affect user satisfaction behavior in the network. As shown in Fig. 7.3, examples of user context variables include user ID, time, location, speed, application, and $\mathrm{QoS}_{\mathrm{d}}$. On the other hand, network context is the set of network variables that affect network conditions, such as noise power, channel gain, Signal to Noise Ratio (SNR), packet rate, and throughput. Taking into consideration user and network context, each user is assigned a set of Resource Blocks (RBs) determined based on the predicted user satisfaction behavior at each particular instant. The resource allocation algorithm will output the optimum set of RBs for each user such that the required satisfaction level is achieved using the minimum amount of resources.

\subsubsection{Problem description}

Usually, resource scheduling problems for networks are modeled as an optimization problem with a single objective that maximizes throughput, spectral efficiency, or fairness under certain constraints. Besides, the objective could be a trade-off between throughput/spectral efficiency and fairness [107]. In this chapter, OPA

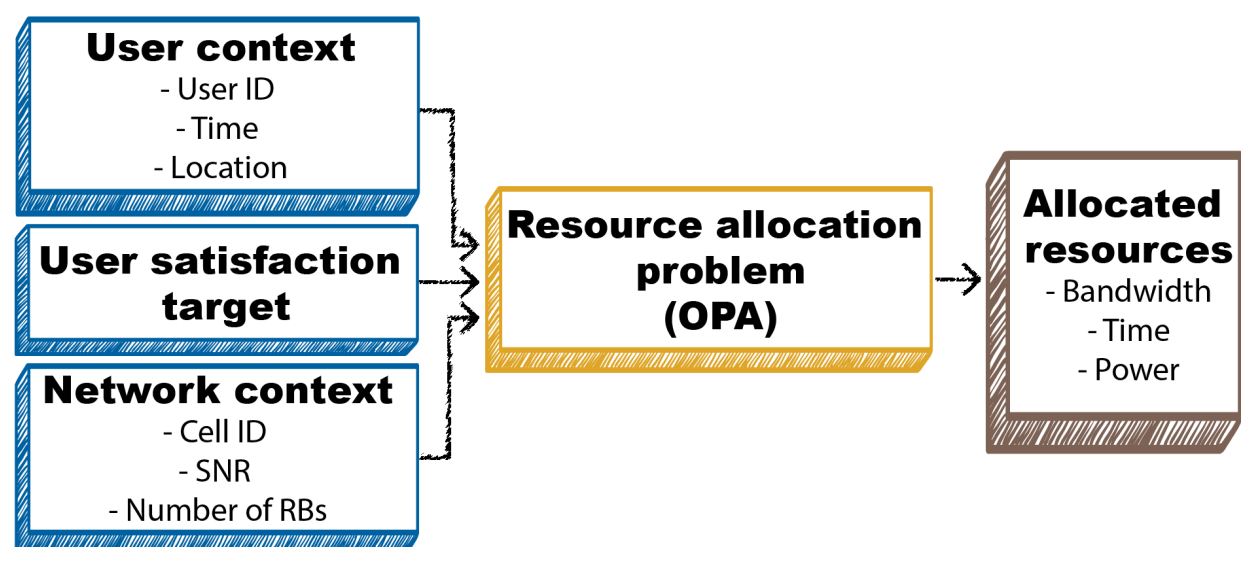

FiguRE 7.3: Inputs and outputs of the Optimum Personalized Resource Allocation (OPA) problem for wireless networks. 
is modeled as a MOO problem. MOO problems are used to model optimization problems with more than one conflicting objective. OPA is defined as a bi-objective optimization problem that maximizes two conflicting objective functions: the total $\Delta\left(\right.$ QoS $\left._{\mathrm{d}^{-}} \mathrm{QoS}_{\mathrm{p}}\right)$ for all users, which represents the amount of saving in network resources, and the average satisfaction for all users. Typically, MOO problems are solved by finding the set of mutually nondominant solutions called the Pareto front. In a Pareto front solution set, there is no solution better than the other as all solutions trade off the conflicting objective functions [108]. Using MOO, along with the available satisfaction information in personalized networks, the network can make granular personalized resource allocation decisions for each user to ensure that the required satisfaction level is achieved with the minimum cost (i.e., resources).

\subsubsection{Problem formulation}

\subsubsection{System model}

let $\mathcal{B}=\{1,2, \ldots, B\}$ eNBs available in the network. The number of user equipments connected to eNB $b$ is denoted by $\mathcal{U}_{b}=\left\{u_{(1, b)}, u_{(2, b)}, \ldots, u_{\left(I_{b}, b\right)}\right\}$, where $I_{b}$ is the number of users connected to eNB $b$. Without loss of generality, $I_{b}$ is assumed constant for all $b \in \mathcal{B}$. For each eNB $b \in \mathcal{B}$, the number of available RBs is denoted by $\mathcal{N}=\left\{n_{1}, n_{2}, \ldots, N\right\} . h_{b, u_{b}}^{(n)}$ is defined, where $u_{b} \in \mathcal{U}_{b}$, as the link gain between eNB $b$ and $u_{b}$ over RB $n$. The power Signal to Interference Noise Ratio (SINR) between eNB $b$ and $u_{b}$ over RB $n$ is as follows:

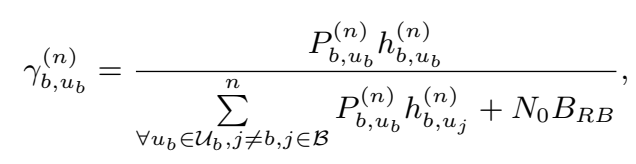

where $P_{b, u_{b}}^{(n)}$ is the power assigned for the link between $b$ and $u_{b}$ over RB $n$ for all $b \in \mathcal{B}, N_{0}$ denotes the thermal noise, and $B_{R B}$ is the resource block bandwidth. The power allocation vector for user $i$ connected to eNB $b$ is $\mathcal{P}_{b, u_{b}}=$ $\left[P_{b, u_{b}}^{(1)}, P_{b, u_{b}}^{(2)}, \ldots, P_{b, u_{b}}^{(N)}\right]$. For simplicity, interference between cells is assumed negligible and SINR for all $b \in \mathcal{B}$ is reduced to the following SNR:

$$
\gamma_{b, u_{b}}^{(n)}=\frac{P_{b, u_{b}}^{(n)} h_{b, u_{b}}^{(n)}}{N_{0} B_{R B}} .
$$

The achievable downlink data rate for all $u_{b} \in \mathcal{U}_{b}$ over $\mathrm{RB} n$ is given by

$$
r_{b, u_{b}}^{(n)}=B_{R B} \log _{2}\left(1+\gamma_{b, u_{b}}^{(n)}\right) .
$$


Chapter 7. Personalized Resource Allocation in Wireless Networks: An AI-Enabled and BD-Driven Multi-Objective Optimization

\subsubsection{Solution encoding: Resource block and power allocation}

The RB allocation indicator is denoted by binary decision variable $x_{u_{b}}^{(n)} \in\{0,1\}$, where

$$
x_{u_{b}}^{(n)}= \begin{cases}1, & \text { if RB } n \text { is assigned to } u_{b} \\ 0, & \text { otherwise }\end{cases}
$$

The RB allocation vector for user $u_{b}$ connected to eNB $b$ is $\mathcal{X}_{u_{b}}=\left[x_{u_{b}}^{(1)}, x_{u_{b}}^{(2)}, \ldots, x_{u_{b}}^{(N)}\right]$. In each frame, the total rate achieved by $u_{b}$ over the allocated RBs is given by

$$
\mathcal{R}_{u_{b}}=\sum_{n=1}^{\mathcal{N}} x_{u_{b}}^{(n)} r_{b, u_{b}}^{(n)}
$$

for all $u_{b} \in \mathcal{U}_{b}$. Consequently, $\Delta_{u_{b}}$, which refers to the amount of saved resources by user $u_{b}$, is given by

$$
\Delta_{u_{b}}=\mathcal{D}_{u_{b}}-\mathcal{R}_{u_{b}},
$$

where $\mathcal{D}_{u_{b}}$ is the rate demanded by user $u_{b}$ connected to eNB $b$. The sum of $\Delta_{u_{b}}$ for all $u_{b} \in \mathcal{U}_{b}$ is given by

$$
\Delta_{\mathcal{U}_{b}}=\sum_{u_{b} \in \mathcal{U}_{b}} \mathcal{D}_{u_{b}}-\mathcal{R}_{u_{b}} .
$$

The total rate achieved by all users connected to eNB $b$ is given by

$$
\mathcal{R}_{b}=\sum_{u_{b} \in \mathcal{U}_{b}} \mathcal{R}_{u_{b}} .
$$

\subsubsection{Data driven optimization}

Let $A_{u_{b}}$ be a $J$-dimensional feature space for $u_{b}$, where $A_{u_{b}}=\left[a_{\left(1, u_{b}\right)}, a_{\left(2, u_{b}\right)}, \ldots, a_{\left(J, u_{b}\right)}\right]$ and $a_{\left(j, u_{b}\right)}$ is the value of feature $j$ for user $u_{b}$. As mentioned earlier, since personalized satisfaction is predicted using the data collected from users, the output of a satisfaction level $\mathcal{S}_{u_{b}}$ is predicted using the deployed and tuned ML-based surrogate model. The inputs for the surrogate model are user ID, context features $A_{u_{b}}$, and user demand $\mathcal{D}_{u_{b}}$.

\subsubsection{Optimization problem formulation}

In order to solve OPA, a MOO problem is formulated with two objectives: I) maximize the average $\Delta_{\mathcal{U}_{b}}$ (i.e., amount of saved resources) for all users and II) maximize the average satisfaction for all users. The formulated MOO has two decision variables: $\mathcal{X}_{u_{b}}$ and $\mathcal{P}_{u_{b}}$. Each objective function is associated with a set of constrained and formulated as follows: 
Chapter 7. Personalized Resource Allocation in Wireless Networks: An AI-Enabled and BD-Driven Multi-Objective Optimization

- Maximize the average $\Delta_{\mathcal{U}_{b}}$ for all users:

$$
\begin{array}{ll}
\max _{\mathcal{X}_{u_{b}}, \mathcal{P}_{u_{b}}} \frac{1}{I_{b}} \sum_{u_{b} \in \mathcal{U}_{b}}\left(\mathcal{D}_{u_{b}}-\sum_{n=1}^{\mathcal{N}} x_{u_{b}}^{(n)} r_{b, u_{b}}^{(n)}\left(P_{b, u_{b}}^{(n)}\right)\right), \\
\text { s.t. } & , \forall n \in N \\
\sum_{u_{b} \in \mathcal{U}_{b}} x_{u_{b}}^{(n)} \leqslant 1 & , \forall u_{b} \in \mathcal{U}_{b} \\
\sum_{u_{b} \in \mathcal{U}_{b}} \sum_{n=1}^{N} x_{u_{b}}^{n} P_{b, u_{b}}^{n} \leqslant \mathcal{P}_{b}^{\max } & \\
\mathcal{R}_{u_{b}, b} \leqslant \mathcal{D}_{u_{b}} & , \forall n \in N, u_{b} \in \mathcal{U}_{b} . \\
P_{b, u_{b}}^{(n)} \geqslant 0 &
\end{array}
$$

- Maximize the average satisfaction for all users:

$$
\begin{aligned}
& \max _{\mathcal{X}_{u_{b}}, \mathcal{P}_{u_{b}}} \frac{1}{I_{b}} \sum_{u_{b} \in \mathcal{U}_{b}} \mathcal{S}_{u_{b}}\left(A_{u_{b}},\right. \\
&\left.,\left(\mathcal{D}_{u_{b}}-\sum_{n=1}^{\mathcal{N}} x_{u_{b}}^{(n)} r_{b, u_{b}}^{(n)}\left(P_{b, u_{b}}^{(n)}\right)\right)\right),
\end{aligned}
$$

s.t.

$$
\begin{array}{ll}
\sum_{u_{b} \in \mathcal{U}_{b}} x_{u_{b}}^{(n)} \leqslant 1 & , \forall n \in N \\
\sum_{u_{b} \in \mathcal{U}_{b}} \sum_{n=1}^{N} x_{u_{b}}^{n} P_{b, u_{b}}^{n} \leqslant \mathcal{P}_{b}^{\max } & \\
\mathcal{R}_{u_{b}, b} \leqslant \mathcal{D}_{u_{b}} & , \forall u_{b} \in \mathcal{U}_{b} \\
P_{b, u_{b}}^{n} \geqslant 0 & , \forall n \in N, u_{b} \in \mathcal{U}_{b} \\
\mathcal{S}_{u_{b}} \geqslant S_{\min , u_{b}} & , \forall u_{b} \in \mathcal{U}_{b} .
\end{array}
$$

The first objective function in (7.9a) maximizes the average $\Delta_{\mathcal{U}_{b}}\left(\bar{\Delta}_{\mathcal{U}_{b}}\right)$ in order to maximize resource-saving in the network. On the other hand, the second objective function in (7.10a) maximizes the average satisfaction for all users. Both objective 
Chapter 7. Personalized Resource Allocation in Wireless Networks: An AI-Enabled and BD-Driven Multi-Objective Optimization

functions contradict each other; hence, the solution set is expected to be a Pareto front, where the optimum points trade-off both objectives.

As for constraints, the first objective function (7.9a) has four constraints, of which constraint $(7.9 \mathrm{~b})$ ensures that each $\mathrm{RB}$ is being used by no more than one user during a single instance. Also, the second constraint (7.9c) prevents each eNB from allocating a total power more than the budget power $\mathcal{P}_{b}^{\max }$. Besides, the third constraint $(7.9 \mathrm{~d})$ limits the rate provided to each user to values less than the demanded rate $\mathcal{R}_{u_{b}, b}$. The fourth constraint (7.9e) ensures that the allocated power for each user $P_{b, u_{b}}^{n}$ is a positive value. On the other hand, the second objective function (7.10a) has five constraints. Constraints (7.10c), (7.10d), (7.10e), and (7.10f) are similar to the constraints associated with the objective function in (7.9a). The last constraint $(7.10 \mathrm{~g})$ maintains a minimum satisfaction specified for each user. This constraint is added to differentiate among the targeted satisfaction levels for different users; hence, enable the network to provide a wider range of service quality levels and pricing policies.

\subsection{EMOO of Resources in Personalized Wire- less Networks}

In this section, the building blocks of MOEAs utilized to solve the proposed OPA problem are presented. For the purpose of this chapter, the implementation of the optimization formulation considers the optimization of the decision variable $\mathcal{X}_{u_{b}}$, whereas $\mathcal{P}_{u_{b}}$ is assigned a constant value for all users.

\subsubsection{Solution encoding}

As mentioned in Section 7.3.2.2, an OPA solution for one user is encoded as a binary vector $\mathcal{X}_{u_{b}}$, which represents a set of RBs available in the networks. With this in mind, the combined solution for all users is an $I_{b} \times N$ matrix in the form of

$$
\mathcal{X}_{s o l}=\left\{\begin{array}{cccc}
x_{u_{(1, b)}}^{(1)} & x_{u_{(1, b)}}^{(2)} & \ldots & x_{u_{(1, b)}}^{(N)} \\
x_{u_{(2, b)}}^{(1)} & x_{u_{(2, b)}}^{(2)} & \ldots & x_{u_{(2, b)}}^{(N)} \\
\ldots & \ldots & \ldots & \ldots \\
x_{u_{\left(I_{b}, b\right)}^{(1)}}^{(1)} & x_{u_{\left(I_{b}, b\right)}^{(2)}}^{(2)} & \ldots & x_{u_{\left(I_{b}, b\right)}^{(N)}}^{(N)}
\end{array}\right\} .
$$

A solution $\mathcal{X}_{u_{b}}$ is feasible if it meets the constraints associated with both objectives (7.9a) and (7.10a). 
Chapter 7. Personalized Resource Allocation in Wireless Networks: An AI-Enabled and BD-Driven Multi-Objective Optimization

\subsubsection{Objective functions}

The proposed OPA problem in Section 7.3.2.4 is a bi-objective optimization problem with two objectives (1) average $\Delta_{\mathcal{U}_{b}}\left(\overline{\Delta_{\mathcal{U}_{b}}}\right)$ in (7.9a) and (2) average satisfaction in (7.10a). Each solution is evaluated in terms of the aforementioned contradicting objectives. In other words, maximizing user satisfaction will require lower $\Delta \mathrm{s}$; hence, minimum resource-saving, and vice versa. The final Pareto front solutions trade-off these contradicting objectives.

\subsubsection{Population intialization}

The population consists of $M$ solutions in the form of $I_{b} \times N$ matrixes. The initial population is generated by drawing the elements of the solution matrixes $\mathcal{X}_{\text {sol }}$ from a random binary uniform distribution.

\subsubsection{Selected MOEAs}

In this chapter, the performance of five MOEAs in solving OPA is investigated. One of the most famous genetic algorithms considered in this chapter is nondominated sorting evolutionary algorithm II (NSGA-II) [109] and its successor NSGA-III [110]. Besides, an indicator-based MOEA called $\varepsilon$-MOEA [111, 112] is tested. Further, SPEA2, which is a multiobjective evolutionary algorithm that incorporates the concept of elitism [112], is tested.

\subsubsection{Evolutionary operators}

In this chapter, binary tournament selection is implemented as the selection operator for all algorithms [113]. As for crossover and mutation, the Half Uniform Crossover (HUX) operator and bit flip are utilized, respectively [114]. Also, the population size used across all experiments is 100 solutions.

\subsubsection{Stoping criteria}

In practice, wireless networks make decisions and perform actions in real-time; therefore, decision time is considered a crucial factor in solving OPA. In EMOO, decision time is proportional to the NFEs. Since OPA is a large scale optimization problem, practical systems are required to implement the appropriate techniques in order to meet the associated time constraint. Considering that meeting the 
Chapter 7. Personalized Resource Allocation in Wireless Networks: An AI-Enabled and BD-Driven Multi-Objective Optimization

wireless networks time constraint is out of the scope of this thesis, the stopping criterion for the implemented simulation is set to a predefined NFEs.

\subsection{Empirical Analysis and Discussion}

In this section, the performance of the considered MOEAs in solving the formulated OPA is evaluated. problem. Then, the best performing MOEA is used to simulate personalized and non-personalized wireless networks in order to compare them in terms of the amount of saved resources and user satisfaction in the network. Also, the impact of errors and uncertainty introduced by the ML surrogate on the performance of MOEAs is studied. Finally, several experiments are conducted to study the complexity and scalability of the proposed optimization problem.

The prototype and simulations in this chapter were done using Python 3.7.6. The DNN model was built using the TensorFlow library. In addition, the scikitlearn library was used for preprocessing the data, whereas seaborn and Matplotlib were used for visualization purposes. Also, MOO is performed using the Platypus library.

\subsubsection{Experimental settings}

\subsubsection{Cellular network environment}

Consider a cell within a cellular network that covers Ottawa, Canada. The cell has one eNB and it is connected to users moving within its coverage area. The area of the cell is divided into a $k * k$ grid. The cellular network environment is simulated using the parameters listed in Table 7.2. The cellular network operator collects context data from users and stores it in a database. The collected data are of two types, real-time user satisfaction levels and context values. Measurements are recorded at each measuring instant. The period between two measuring instances is referred to as a Time Slot (TS). The service provider collects data from the users using a TS length of one second. Besides, the amount of resources consumed within each TS is recorded. Also, for the sake of simplicity, all users are assumed to have the same minimum requirement for user satisfaction. 
Chapter 7. Personalized Resource Allocation in Wireless Networks: An AI-Enabled and BD-Driven Multi-Objective Optimization

TABLE 7.2: Cellular network simulation parameters.

\begin{tabular}{ll}
\hline Parameter name & Parameter value \\
\hline Maximum number of available RBs & 100 \\
Number of subcarriers per RB & 12 \\
RB bandwidth $\left(B_{R B}\right)$ & $180 \mathrm{kHz}$ \\
Carrier frequency & $2 \mathrm{GHz}$ \\
UE thermal noise density & $-174 \mathrm{dBm} / \mathrm{Hz}$ \\
Grid size $(k)$ & 100 \\
Flat fading & Rayleigh \\
Number of users $\left(I_{b}\right)$ & 4 \\
Number of eNBs & 1 \\
$S_{\text {min }, u_{b}}$ & 4 \\
$P^{\text {max }}$ & 1 Watt \\
\hline
\end{tabular}

\subsubsection{Performance metrics}

The deisgn of MOO metrics ususally considers three main performance criteria: capacity, convergence, and diversity [115]. Capacity metrics quantify the ratio (or number) of nondominated solutions in the solution space $\mathbb{S}$ that conforms to the predefined reference set. To measure MOO performance in terms of capacity, the Overall Non-dominated Vector Generation Ratio (NGR) is calculated [116]. NGR describes the capacity ratio of $\mathbb{S}$ with respect to $\mathbb{R}$, and is given as

$$
N G R(\mathbb{S}, \mathbb{R})=\frac{|\mathbb{S}|}{|\mathbb{R}|}
$$

where |.| is the cardinality or number of elements in the set. In contrast to capacity, convergence metrics measures the proximity of the solution set $\mathbb{S}$ to the reference set $\mathbb{R}$. To measure MOO performance in terms of convergenece, the Generational Distance (GD) is calculated [109] as follows:

$$
G D(\mathbb{S}, \mathbb{R})=\frac{\left(\sum_{i=1}^{|\mathbb{S}|} d_{i}^{2}\right)^{\frac{1}{2}}}{|\mathbb{S}|}
$$

where $d_{i}$ is the smallest distance from $s \in \mathbb{S}$ to the closest solution in $\mathbb{R}$, and is given as $d_{i}=\min _{r \in \mathbb{R}}\left\|F\left(s_{i}\right)-F(r)\right\|$, where $s_{i} \in \mathbb{S}$. $\|$.$\| denotes the Euclidean$ distance and $F=\left(f_{1}(s), f_{2}(s)\right)$, where $f_{1}$ is defined in (7.9a) and $f_{2}$ is defined 
Chapter 7. Personalized Resource Allocation in Wireless Networks: An AI-Enabled and BD-Driven Multi-Objective Optimization

in (7.10a). As for measuring the performance of MOO in terms of diversity, the Spacing (SP) metric [117] is calculated as follows:

$$
S P(\mathbb{S})=\sqrt{\sum_{i=1}^{|\mathbb{S}|} \frac{\left(\left(\bar{d}-d_{i}\right)^{2}\right.}{|\mathbb{S}-1|}} .
$$

In addition to the aforementioned metrics, the Hypervolume (HV) [118] and the Inverted Generational Distance (IGD) are calculateed [119, 120]. HV and IGD measure the performance of MOO in terms of both convergence and diversity. HV is one of the most popular performance metrics for MOO, where it quantifies the volume in the objective space that is dominated by the solution set $\mathbb{S}$. $\mathrm{HV}$ is calculated as follows:

$$
H V(\mathbb{S}, \mathrm{R})=\operatorname{volume}\left(\bigcup_{\mathrm{i}=1}^{|\mathbb{S}|} \mathrm{v}_{\mathrm{i}}\right),
$$

where $v_{i}$ is the hypercube associated with $s_{i} \in \mathbb{S}$, and $R$ is a reference point. On the other hand, IGD is calculated as follows:

$$
\operatorname{IGD}(\mathbb{S},|\mathbb{R}|)=\frac{\left(\sum_{i=1}^{|P|} d_{i}^{2}\right)^{\frac{1}{2}}}{|\mathbb{R}|}
$$

It is worth noting that the goal is to maximize HV and SP, whereas GD and IGD are better when they are minimized.

\subsubsection{Reference set generation}

In order to evaluate the performance and the quality of the Pareto front solutions, it is essential to compare them to a Reference set $\mathbb{R}$, which is the Pareto optimal set [108]. Since the optimal set is not known, the common practice of using the best-known approximation of the Pareto optimal set as the reference set is employed. The approximation of the optimal Pareto set for each instance is performed using the MOEAs listed in Section 7.4.4. Each MOEA is run 30 times and the final Pareto front solutions are merged into one set. Then, the set of merged Pareto front solutions is utilized to extract the set of non-dominated solutions, which represents the approximated Pareto front reference set $\mathbb{R}$. Using the simulation parameters in Table 7.2 , both objectives, the average $\Delta_{\mathcal{U}_{b}}\left(\overline{\Delta_{\mathcal{U}_{b}}}\right)$ vs. the average satisfaction for a single exemplary instance are plotted. For illustrative purposes, the minimum satisfaction allowed in the network is set to $S_{\min , u_{b}}=1$. Fig. 7.4 illustrates the generated solution sets by NSGAII, NSGAIII, SPEA2, and 
Chapter 7. Personalized Resource Allocation in Wireless Networks: An AI-Enabled and BD-Driven Multi-Objective Optimization

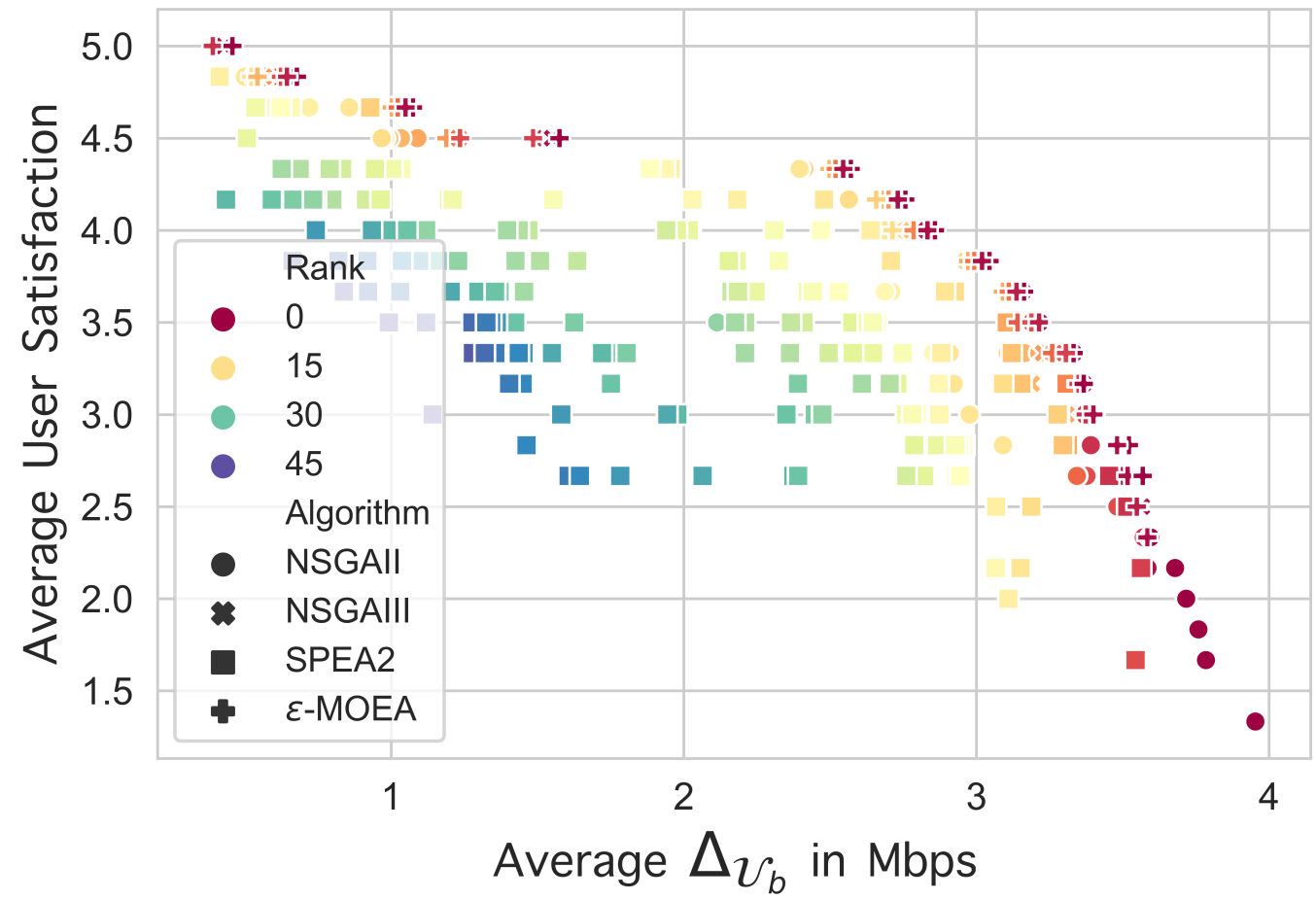

Figure 7.4: The generated approximation sets of the Pareto front solutions by the considered MOEA algorithms.

$\varepsilon$-MOEA and their computed ranks. The Pareto front solutions are the set of solutions in the merged solution set that have the minimum rank (i.e., rank equal to zero), which are referred to as the non-dominated solutions set. In Fig. 7.5, the extracted optimum Pareto front solution set is plotted. The Pareto front solutions provide a trade-off between both objectives. Lower $\overline{\Delta_{\mathcal{U}_{b}}}$ values (i.e., a higher amount of consumed resources) offer higher user satisfaction levels in the network. To achieve a certain satisfaction level in the network, the optimum Pareto front solution for each instance is used to find the required minimum amount of resources. Depending on the service provider policy and the required $S_{\min , u_{b}}$, the personalized network will operate at the Pareto front solution point that achieves the required average satisfaction using the minimum resources. For the instance depicted in Fig. 7.5, if the required average satisfaction level for all users is $4, \bar{\Delta}_{\mathcal{U}_{b}}$ should be less than or equal to 2.9 , which is the maximum (i.e., optimum) $\bar{\Delta}_{\mathcal{U}_{b}}$ solution. 


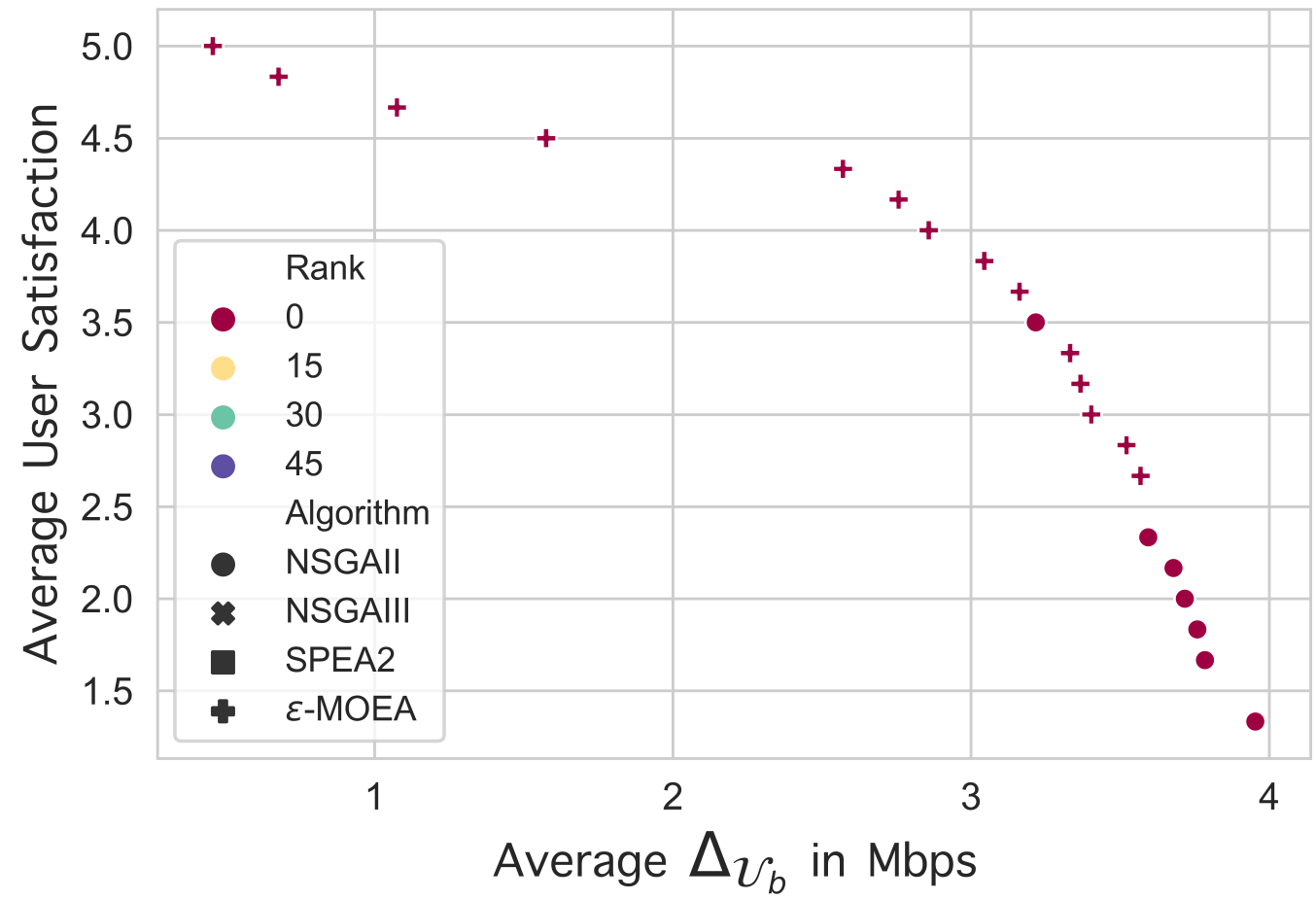

Figure 7.5: The non-dominated Pareto front reference solution set extracted from the multiple sets generated by the considered MOEA algorithms.

\subsubsection{Experiment 1: statistical analysis of MOEAs perfor- mance results}

In this experiment, the performance of the considered MOEAs is evaluated and compared in terms of the metrics described in Section 7.5.1.2. Since some of the performance metrics use the reference set $\mathbb{R}$ as a parameter, an approximation of the reference set is computed for each instance. In order to test the significance of the performance results obtained in this section, non-parametric tests are used [121]. First, the Friedman $N$ x $N$ procedure is utilized to validate the existence of statistical differences among the results obtained by all algorithms. The Friedman test examines the null hypothesis $\left(H_{0}\right)$ that the performance results for all algorithms come from the same distribution. In this chapter, a significance level $(\alpha)$ of 0.05 is picked. This means that if $p$-value is less than 0.05 , the $H_{0}$ is rejected; hence, there exist statistical differences between the algorithms' performance results. Otherwise, $H_{0}$ can not be rejected and the samples are likely coming from the same statistical distribution. If the Friedman test suggests the rejection of $H_{0}$, several posthoc tests are performed to examine the statistical difference of each algorithm from every other algorithm. The performed posthoc tests are Conover, Wilcoxon, Nemenyi, and Mann-Whitney [122]. 
In this experiment, to ensure the consistent performance of the chosen algorithm across all instances, a number of instances $N_{m}$ are randomly chosen from the WPP dataset described in Chapter 4. For each instance, MOEAs described in Section 7.4.4 are run to solve the formulated OPA problem. Then, the performance metrics described in Section 7.5.1.2 are computed. To ensure the statistical significance of the performance results for each instance, this process is repeated $N_{s}$ times for each instance in the selected $N_{m}$ instance set. Thereafter, the mean of each performance metric and for each algorithm is computed over all $N_{s}$ runs. The computed mean data is used to compare the algorithms.

\subsubsection{Sample size sufficiency}

Before proceeding with the performance results comparison, it is important to determine whether the sample size is large enough to support the experiments. This has to do with the fact that too small sample size may produce inconclusive results. In literature, different sample sizes are used, yet a clear justification of the selection is rarely provided. One approach to choose a sufficient sample size $N_{m}$ and $N_{s}$ is to examine their relationship with the Standard Error of the Mean $\left(S E_{M}\right)$, given as

$$
S E_{M}=\frac{\sigma}{\sqrt{n}},
$$

where $\sigma$ is the sample standard deviation and $n$ is the number of samples [123]. For the purpose of this chapter, a maximum $S E_{M}$ of 0.05 is chosen. In Fig. 7.6.a and Fig. 7.6.b, the $S E_{M}$ is computed and plotted for the sample sizes $N_{m}$ and $N_{s}$, respectively. Using the $S E_{M}$ data in Fig. 7.6.a and Fig. 7.6.b, the value of 30 samples is assigned to $N_{s}$ and $N_{m}$, which achieves $S E_{M}$ lower than 0.05 .

\subsubsection{Statistical analysis}

Using the picked $N_{s}$ and $N_{m}$ values, the described statistical analysis experiment is performed. Table 7.3 summarizes the statistical and Friedman test results of the performed experiment. The Friedman test results show that the $p$-value for all performance metrics are less than $\alpha=0.05$; hence, the test rejects $H_{0}$ and accepts the alternative hypothesis $H_{a}$. Consequently, Friedman test results suggest that, for each performance metric, there is a significant statistical difference among the metric values calculated for all algorithms. 
Chapter 7. Personalized Resource Allocation in Wireless Networks: An AI-Enabled and BD-Driven Multi-Objective Optimization

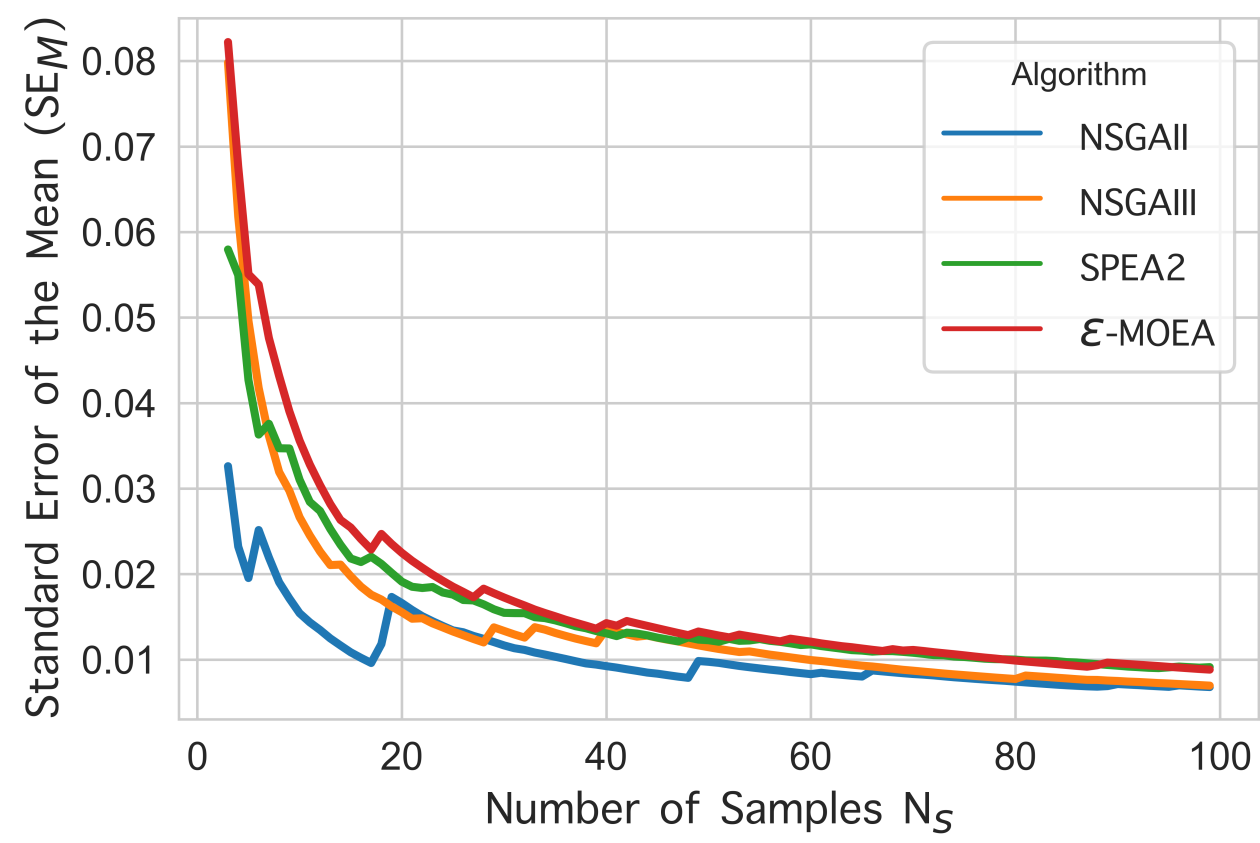

(A) $S E_{M}$ vs. sample size $N_{s}$.

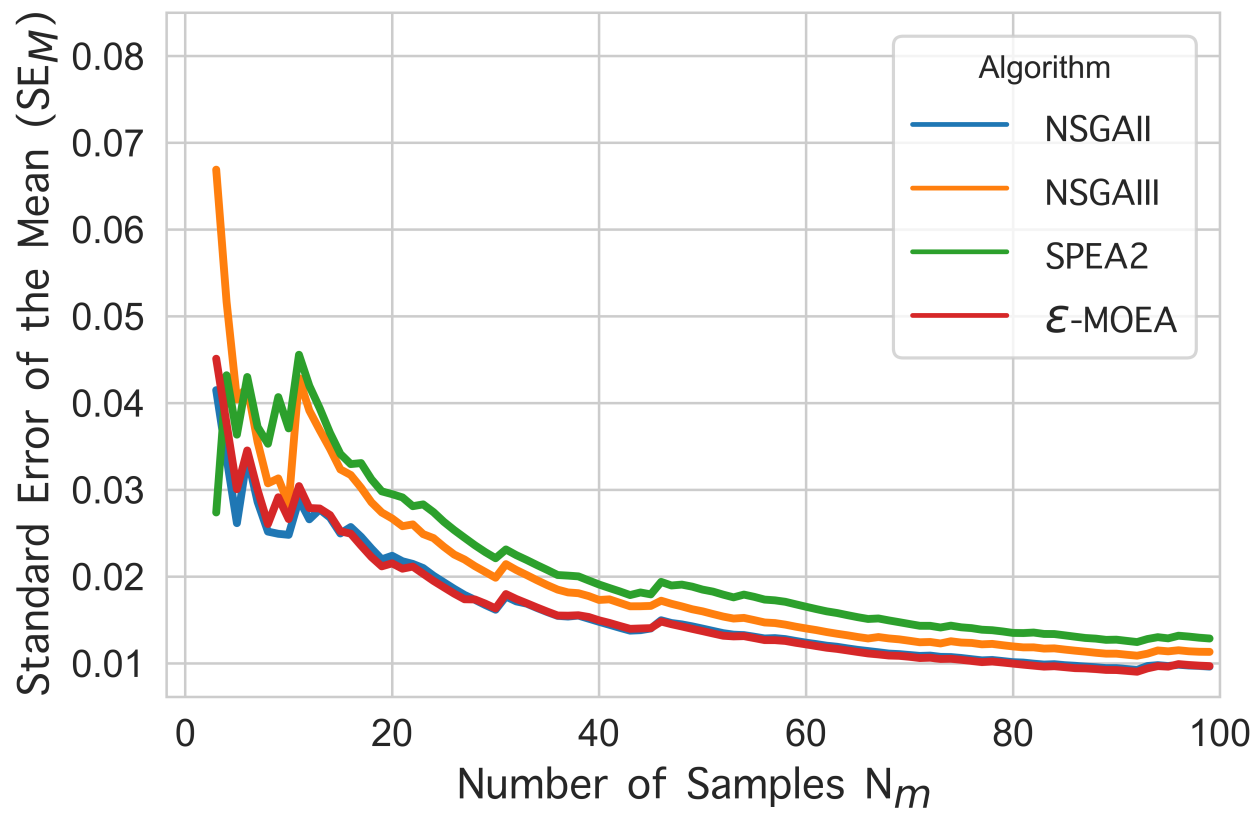

(B) $S E_{M}$ vs. sample size $N_{m}$.

Figure 7.6: Standard Error of the Mean $\left(S E_{M}\right)$ vs. sample sizes. 
Chapter 7. Personalized Resource Allocation in Wireless Networks: An AI-Enabled and BD-Driven Multi-Objective Optimization

TABLE 7.3: Statistical and Friedman test results.

\begin{tabular}{|c|c|c|c|c|c|c|c|}
\hline \multirow{2}{*}{ Metric } & & \multicolumn{4}{|c|}{ Algorithms } & \multirow{2}{*}{$p$-value } & \multirow{2}{*}{ Statistic } \\
\hline & & NSGAII & NSGAIII & SPEA2 & $\varepsilon$-MOEA & & \\
\hline \multirow{4}{*}{ HV } & Rank & 3.520 & 3.880 & 1.970 & 4.580 & \multirow{4}{*}{$<0.001$} & \multirow{4}{*}{336.184} \\
\hline & Mean & 0.504 & 0.507 & 0.367 & 0.535 & & \\
\hline & Max & 0.721 & 0.723 & 0.629 & 0.722 & & \\
\hline & Min & 0.213 & 0.199 & 0.012 & 0.266 & & \\
\hline \multirow{4}{*}{ GD } & Rank & 1.460 & 2.600 & 4.940 & 4.000 & \multirow{4}{*}{$<0.001$} & \multirow{4}{*}{331.808} \\
\hline & Mean & 0.013 & 0.019 & 0.098 & 0.038 & & \\
\hline & Max & 0.051 & 0.054 & 0.048 & 1.086 & & \\
\hline & Min & 0.004 & 0.005 & 0.010 & 0.007 & & \\
\hline \multirow{4}{*}{ IGD } & Rank & 2.640 & 2.570 & 4.120 & 1.720 & \multirow{4}{*}{$<0.001$} & \multirow{4}{*}{164.392} \\
\hline & Mean & 0.221 & 0.217 & 0.318 & 0.192 & & \\
\hline & Max & 0.669 & 0.578 & 1.307 & 0.558 & & \\
\hline & Min & 0.070 & 0.054 & 0.077 & 0.049 & & \\
\hline \multirow{4}{*}{ SP } & Rank & 3.120 & 2.080 & 3.985 & 4.810 & \multirow{4}{*}{$<0.001$} & \multirow{4}{*}{363.668} \\
\hline & Mean & 11.039 & 6.036 & 16.440 & 26.232 & & \\
\hline & Max & 19.728 & 16.882 & 33.966 & 69.815 & & \\
\hline & Min & 4.739 & 1.184 & 0.000 & 3.267 & & \\
\hline \multirow{4}{*}{ NGR } & Rank & 3.980 & 2.145 & 5.000 & 2.875 & \multirow{4}{*}{$<0.001$} & \multirow{4}{*}{388.476} \\
\hline & Mean & 1.168 & 0.627 & 8.094 & 0.675 & & \\
\hline & Max & 2.539 & 0.873 & 15.940 & 0.910 & & \\
\hline & Min & 0.716 & 2.590 & 0.077 & 9.206 & & \\
\hline \multicolumn{2}{|c|}{ API } & -3.472 & 2.303 & -5.597 & 3.611 & & \\
\hline
\end{tabular}

In order to select the best performing algorithm, the evaluated ranks by the Friedman test are employed to compute a new metric, which are refered to as the Algorithm Performance Indicator (API), defined as follows:

$$
\begin{aligned}
A P I= & \left|w_{H V}\right| e^{j \theta_{H V}} * H V_{r}+\left|w_{S P}\right| e^{j \theta_{S P}} * S P_{r}+ \\
& \left|w_{N G R}\right| e^{j \theta_{N G R}} * N G R_{r}+\left|w_{G D}\right| e^{j \theta_{G D}} * G D_{r} \\
& +\left|w_{I G D}\right| e^{j \theta_{I G D}} * I G D_{r},
\end{aligned}
$$

where $H V_{r}, N G R_{r}, G D_{r}, I G D_{r}, S P_{r}$ are the algorithm ranks of $\mathrm{HV}$, NGR, GD, IGD, and SP. Besides, $\left|w_{i}\right|$ and $\theta_{i} \forall i \in[H V, S P, N G R, G D, I G D]$ are the magnitude and phase of the weight $w_{i}$. The weights magnitude can be chosen based on the importance of each metric to the requirements of the tackled problem. 
Chapter 7. Personalized Resource Allocation in Wireless Networks: An AI-Enabled and BD-Driven Multi-Objective Optimization

TABle 7.4: Pairwise comparison of algorithms rejected by the posthoc tests.

\begin{tabular}{|c|c|c|c|c|c|}
\hline Algorithms & HV & GD & IGD & SP & NGR \\
\hline NSGAII vs. NSGAIII & & & & $\sqrt{ }$ & $\sqrt{ }$ \\
\hline NSGAII vs. SPEA2 & $\sqrt{ }$ & & $\sqrt{ }$ & $\sqrt{ }$ & $\sqrt{ }$ \\
\hline NSGAII vs. $\varepsilon$-MOEA & $\sqrt{ }$ & & $\sqrt{ }$ & $\sqrt{ }$ & $\sqrt{ }$ \\
\hline NSGAIII vs. SPEA2 & $\sqrt{ }$ & $\sqrt{ }$ & $\sqrt{ }$ & $\sqrt{ }$ & $\sqrt{ }$ \\
\hline NSGAIII vs. $\varepsilon$-MOEA & $\sqrt{ }$ & $\sqrt{ }$ & $\sqrt{ }$ & $\sqrt{ }$ & $\sqrt{ }$ \\
\hline 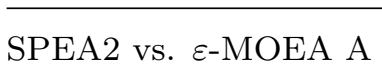 & $\sqrt{ }$ & & $\sqrt{ }$ & $\sqrt{ }$ & $\sqrt{ }$ \\
\hline
\end{tabular}

Nonetheless, for the purpose of this chapter, the same weight value of $1 / 5$ is assigned to all weights; hence, all indicators are assumed to be of equal importance in the final score. As mentioned earlier, some metrics are better when their values are higher and others are the opposite. To reflect this in the API score, $\theta=0$ is assigned for the metrics that are better when they are higher (i.e., HV, SP) and $\theta=\pi$ for those who are better when they are lower (i.e., GD, IGD). In comparison to other metrics, NGR could have $\theta=0$ or $\pi$ depending on the value of $N G R_{r}$. This is because $N G R_{r}<1$ is indicative of missing non-detected Pareto front solutions, wheres $N G R_{r}>1$ is indicative of a higher number of detected solutions compared to the reference set solutions $\mathbb{R}$. Therefore, the closer NGR to 1 , the better the quality of the Pareto front solutions. To incorporate this in the API equation, $\theta_{N G R}$ is defined by

$$
\theta_{N G R}=(1-\beta) \pi
$$

where $\beta$ is given by

$$
\beta=\left\{\begin{array}{ll}
0 & , N G R>1 \\
1 & , N G R \leq 1
\end{array} .\right.
$$

Table 7.3, lists the API score for each algorithm. Of the four algorithms, $\varepsilon$-MOEA achieved the highest score of 3.611; therefore, it will be used for the personalized network simulations in the subsequent experiments.

Before accepting the aforementioned results, it is important to verify that the statistics for $\varepsilon$-MOEA are significantly different for the other algorithms. To test that, the described pairwise posthoc tests are performed. Table 7.4 summarizes the posthoc tests results. Although the posthoc tests failed to reject $H_{0}$ for some 
Chapter 7. Personalized Resource Allocation in Wireless Networks: An AI-Enabled and BD-Driven Multi-Objective Optimization

of the metrics computed for NSGAII and NSGAIII, $\varepsilon$-MOEA showed a significant statistical difference from every other algorithm.

\subsubsection{Experiment 2: personalized vs. non-personalized wireless networks}

The goal of this experiment is to provide insights into the dynamics of personalized wireless networks and to show how they can be used to save the scarce network resources and improve user satisfaction levels in a controlled manner. Besides, the behavior of Surrogate-assisted Personalized Wireless Networks (SPN) is compared to Direct Feedback Personalized Wireless Networks (FPN) (i.e., networks utilizing direct user satisfaction feedback). Although the latter approach is not practical, it is used as a benchmark to study how the user satisfaction surrogates can deteriorate the optimum solutions of OPA, and consequently the amounts of savings and user satisfaction levels in the network. Also, both SPN and FPN are compared to the Non-Personalized Network (NPN), which tries to maximize the utilization of the available resources and maximize the provided rate. The wireless networks simulated in this section have four active users and NFEs is set to 5000 evaluations. Besides, the simulation time frame is set to 50 minutes. The networks described in this section are simulated at a resolution of one second (i.e., $\mathrm{TS}=1$ second); hence, the optimization of OPA is run at every second within the simulation time frame. Nonetheless, for visualization purposes, the results are averaged over every 30 seconds. Notably, the simulated SPN does not employ the surrogate management framework illustrated in Fig. 7.2.

The first promised advantage of personalized networks is saving resources compared to current wireless networks. The amount of saved resources for both SPN and FPN is measured by $\mathrm{Q}_{\mathrm{NP}}-\mathrm{Q}_{\mathrm{N}} \mathrm{S}_{\mathrm{P}}$, where $\mathrm{Q}_{\mathrm{N}} \mathrm{S}_{\mathrm{NP}}$ is the QoS provided by the non-personalized network and $\mathrm{Q}_{\mathrm{O}} \mathrm{S}_{\mathrm{P}}$ is the QoS provided by the personalized network, both in Megabits per seconds (Mbps). Fig. 7.7.a compares the amount of saved resources for FPN and SPN. The first thing that can be observed is the similar trends and patterns between both networks, which is indicative of the validity of the produced solutions by the surrogate-assisted OPA problem. Also, it can be further observed that the SPN spends fewer resources compared to the FPN; consequently, SPN achieved higher resource-savings. With this in mind, the amount of saved resources by the FPN is the maximum achievable amount of saved resources that doesn't compromise the required satisfaction levels required by the network operator. As mentioned earlier, another promised advantage of 
Chapter 7. Personalized Resource Allocation in Wireless Networks: An AI-Enabled and BD-Driven Multi-Objective Optimization

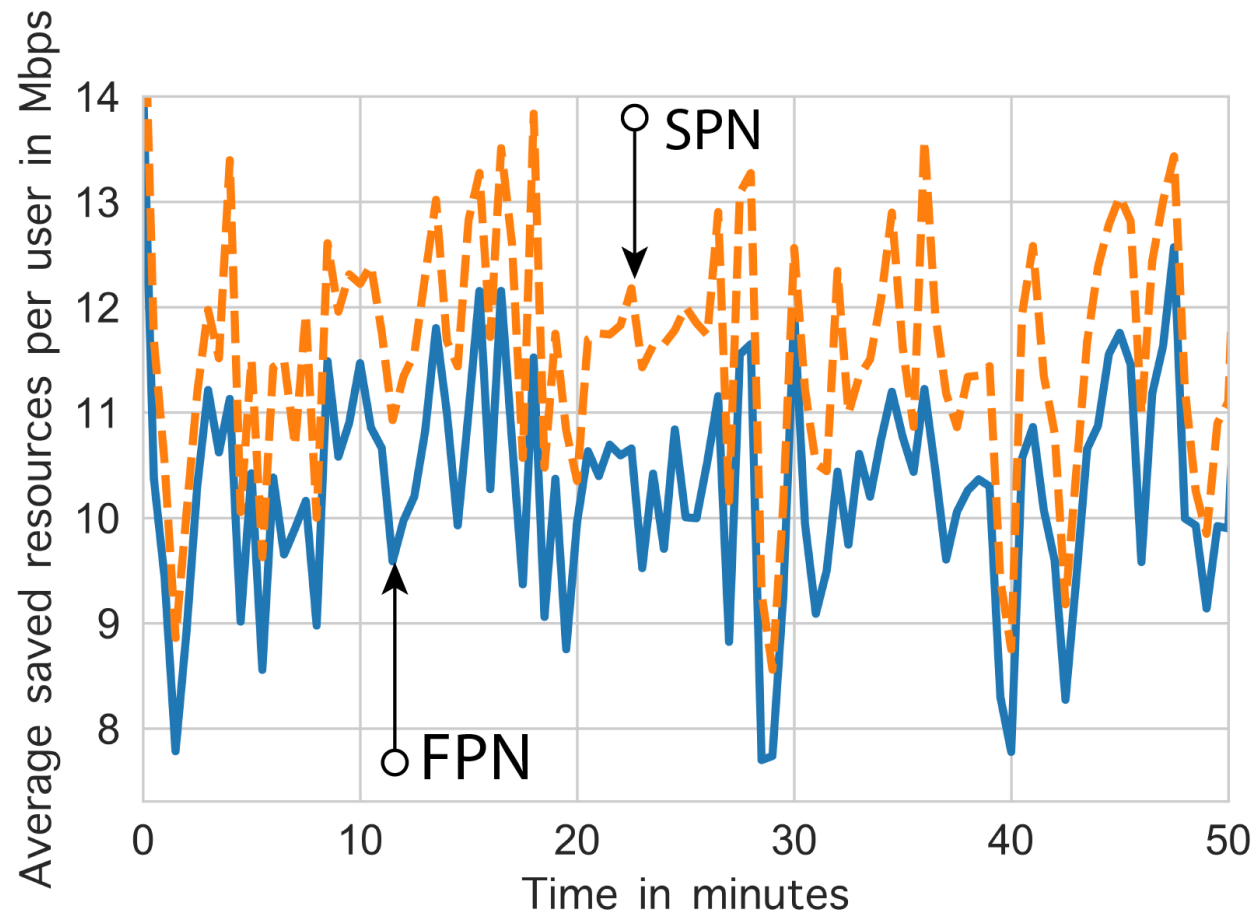

(A) The amount of saved resources $\left(Q_{0} S_{N P}-\right.$ QoSP $)$ by FPN and SPN for the four users vs. time in minutes.

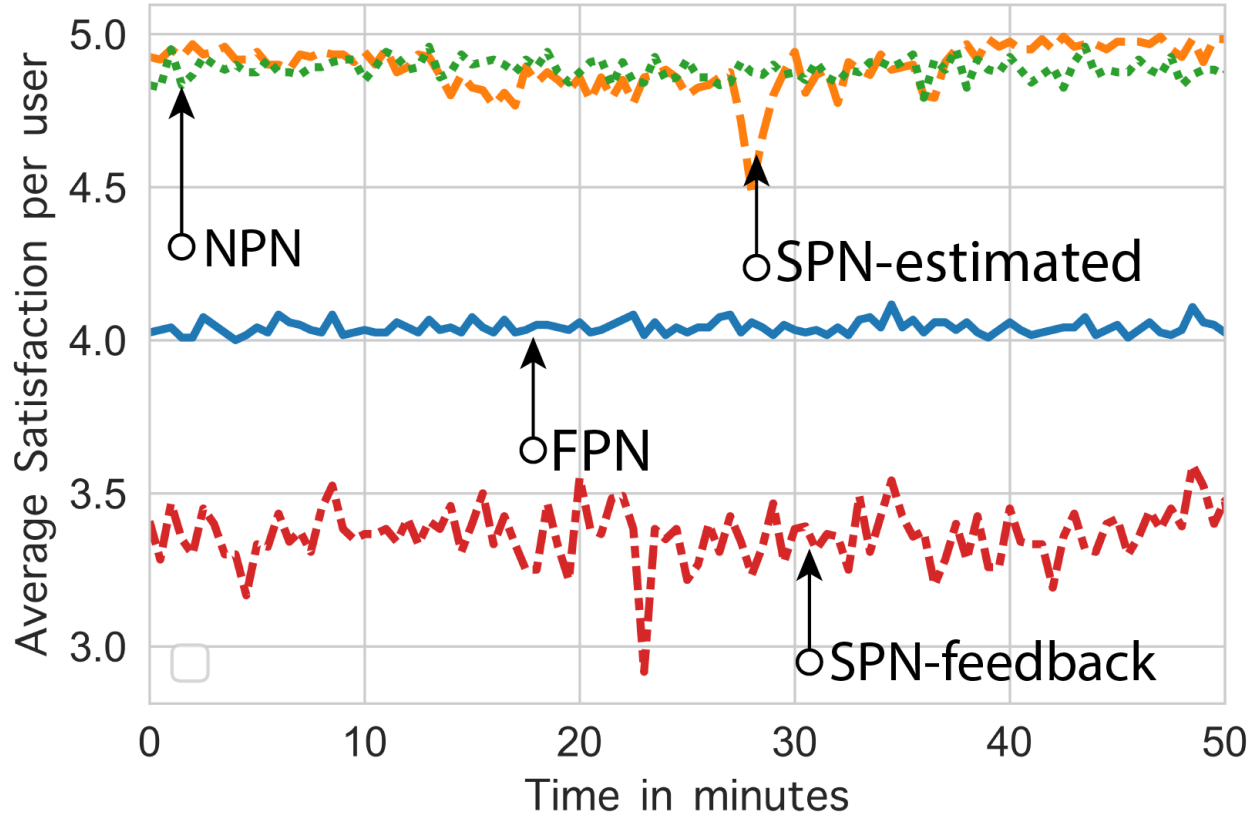

(B) Average user satisfaction for the four users vs. time in minutes for SPN and FPN.

Figure 7.7: The simulation results of the Direct Feedback Personalized Network (FPN) and the Surrogate-assisted Personalized Network (SPN) for four network users. 
Chapter 7. Personalized Resource Allocation in Wireless Networks: An AI-Enabled and BD-Driven Multi-Objective Optimization

personalized networks is maintaining a specific user satisfaction level. In order to analyze the user satisfaction levels achieved by the simulated three networks, Fig. 7.7.b compares the average satisfaction levels for the four users vs. time for NPN, FPN. Also, for SPN, the average satisfaction levels predicted by the surrogate model (SPN-estimated) are plotted. In order to benchmark the predicted satisfaction results, the actual satisfaction levels measured using direct user feedback (SPN-feedback) are plotted. The first thing to observe from 7.7.b is that the satisfaction levels for NPN and FPN networks are above the specified minimum of $S_{\min , u_{b}}=4$. Perhaps the most important observation from Fig. 7.7.b is the gap between the estimated (SPN-estimated) and the actual satisfaction levels for SPN (SPN-feedback). Although the SPN achieved superior amounts of saved resources compared to FPN (see Fig. 7.7.a), it failed to achieve the required average satisfaction level of 4 . This is due to the satisfaction uncertainty introduced by the surrogate model, which led the SPN to further reduce resources below the minimum required to achieve $S_{\min , u_{b}}=4$. These findings emphasize the importance of an effective surrogate management strategy to avoid the deterioration and divergence of user satisfaction levels resulting from false satisfaction predictions in the network.

\subsubsection{Experiment 3: the impact of uncertainty introduced by surrogates on the performance of MOEAs}

Generally, as shown in the previous experiment, the estimation error introduced by surrogates impacts the network's ability to use accurate user satisfaction information in the optimization process. The magnitude of this impact depends on several factors of which the most important is the performance of the utilized surrogate model. To further study this assumption, the following experiment is designed and performed. In order to vary the performance level of the surrogate, the amount of training data is gradually increased. The accuracy and the amount of data used for training are recorded for each surrogate model. Then, using the set of trained surrogate models, the MOEAs are run to solve the OPA problem. For each surrogate model, each MOEA is run for 30 times; thereafter the average HV is computed. Fig. 7.8 compares the average HV values for the surrogate models with varying performance levels. Essentially, as shown in Fig. 7.8, as the quality of the employed surrogate model improves, the quality of the OPA solutions for all algorithms improve with different levels. 
Chapter 7. Personalized Resource Allocation in Wireless Networks: An AI-Enabled and BD-Driven Multi-Objective Optimization

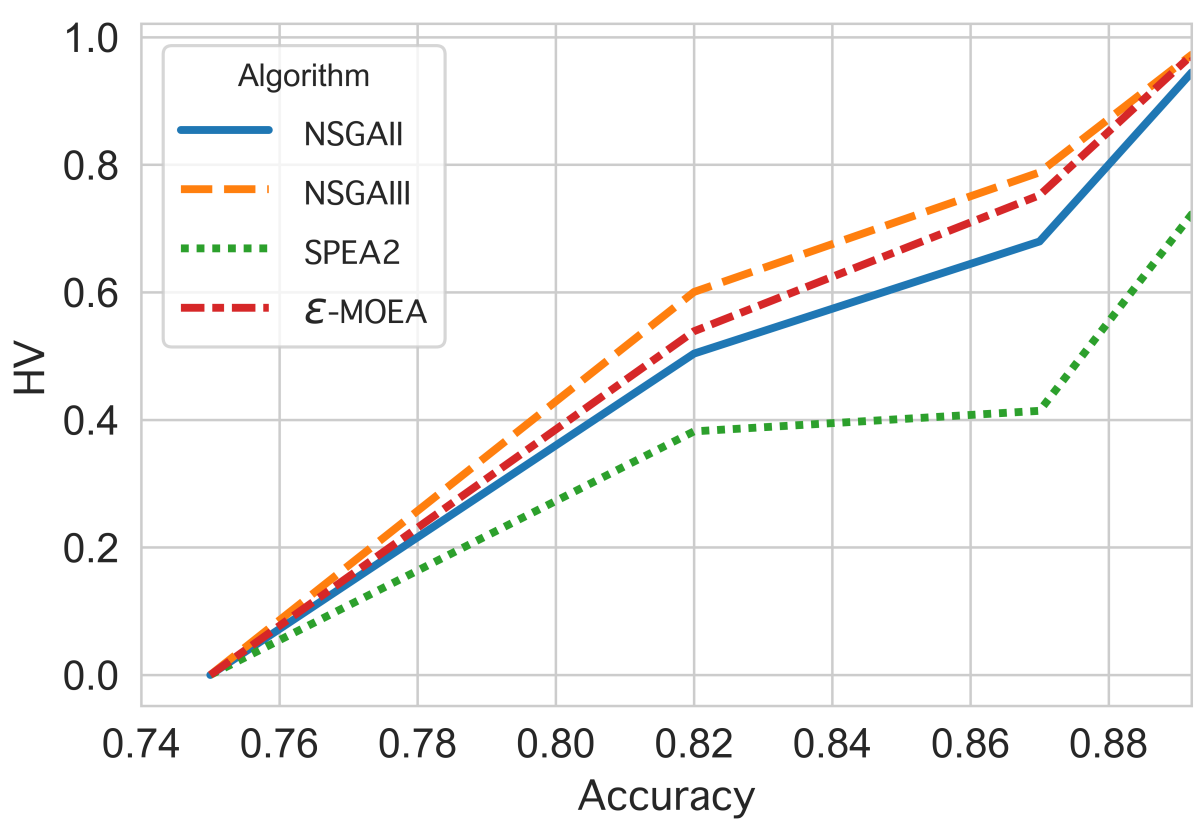

FiguRE 7.8: The average HV computed for different surrogate models with varying performance levels for NSGAII, NSGAIII, SPEA2, and $\varepsilon$-MOEA.

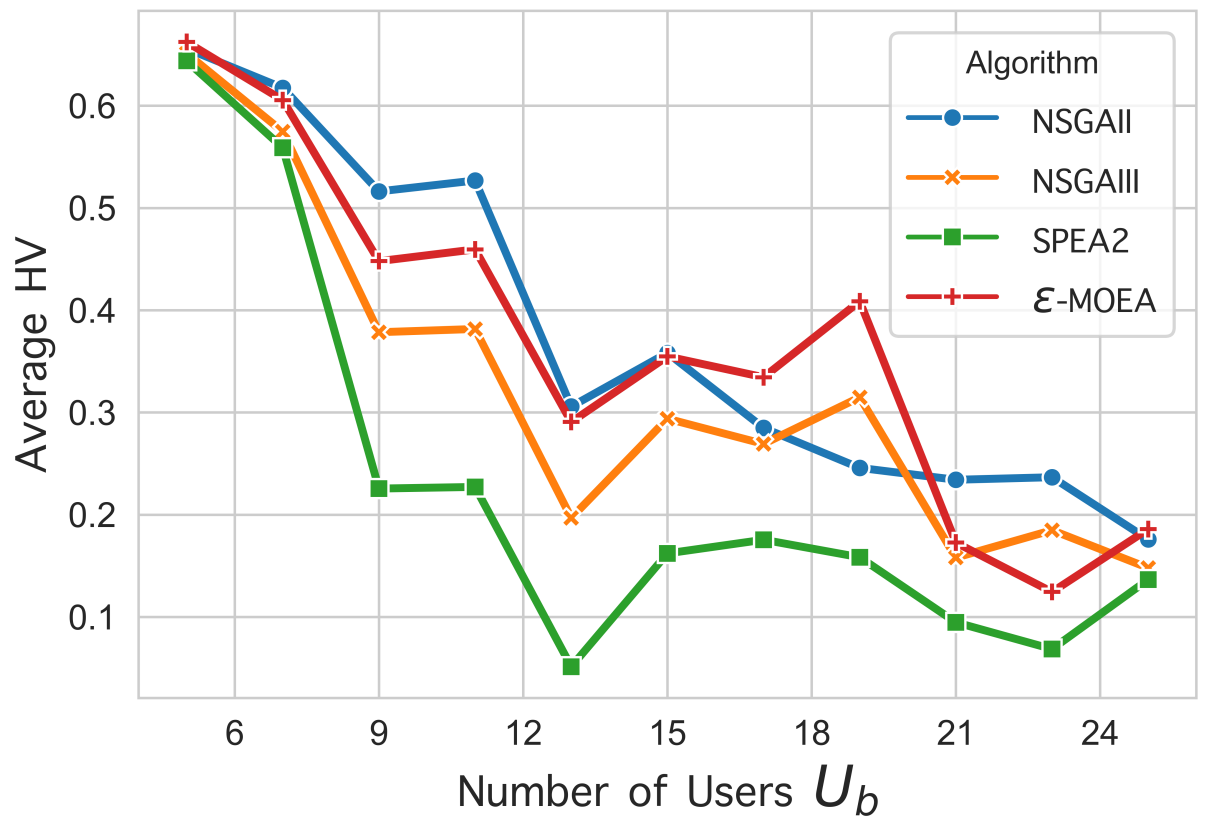

Figure 7.9: The number of users $\mathcal{U}_{b}$ vs. average HV for NSGAII, NSGAIII, SPEA2, and $\varepsilon$-MOEA. 
Chapter 7. Personalized Resource Allocation in Wireless Networks: An AI-Enabled and BD-Driven Multi-Objective Optimization

\subsubsection{Experiment 4: scalability Analysis}

In order to evaluate the scalability of the proposed formulation, the effect of variables that contribute to the complexity of the problem is explored. For the formulated problem in this chapter, the number of users $\mathcal{U}_{b}$ determines the size of the problem decision variables; therefore, it increases the complexity of the problem. Another factor that impacts the complexity of the problem is the required quality of solutions. Higher quality solutions usually require higher NFEs; thereby, higher amounts of computing resources. In this section, the effect of $\mathcal{U}_{b}$ and NFEs on complexity is explored. Due to the random nature of the simulated wireless network environment, and to make a fair comparison among the considered algorithms, the simulation environment values are unified across all algorithms for a certain simulation instance.

\subsubsection{The impact of the number of network users on complexity}

To study the effect of the number of network users $\mathcal{U}_{b}$ on OPA, the following experiment is performed. Using a random instance, the MOEAs considered in this chapter are run 30 times for varying number of users $\mathcal{U}_{b}$. NFEs is set to 5000 evaluations for each run. Then, $\mathrm{HV}$ is computed and averaged over the 30 runs for each $\mathcal{U}_{b}$. Fig. 7.9 depicts the averaged $H V$ values vs. the number of users $\mathcal{U}_{b}$ for

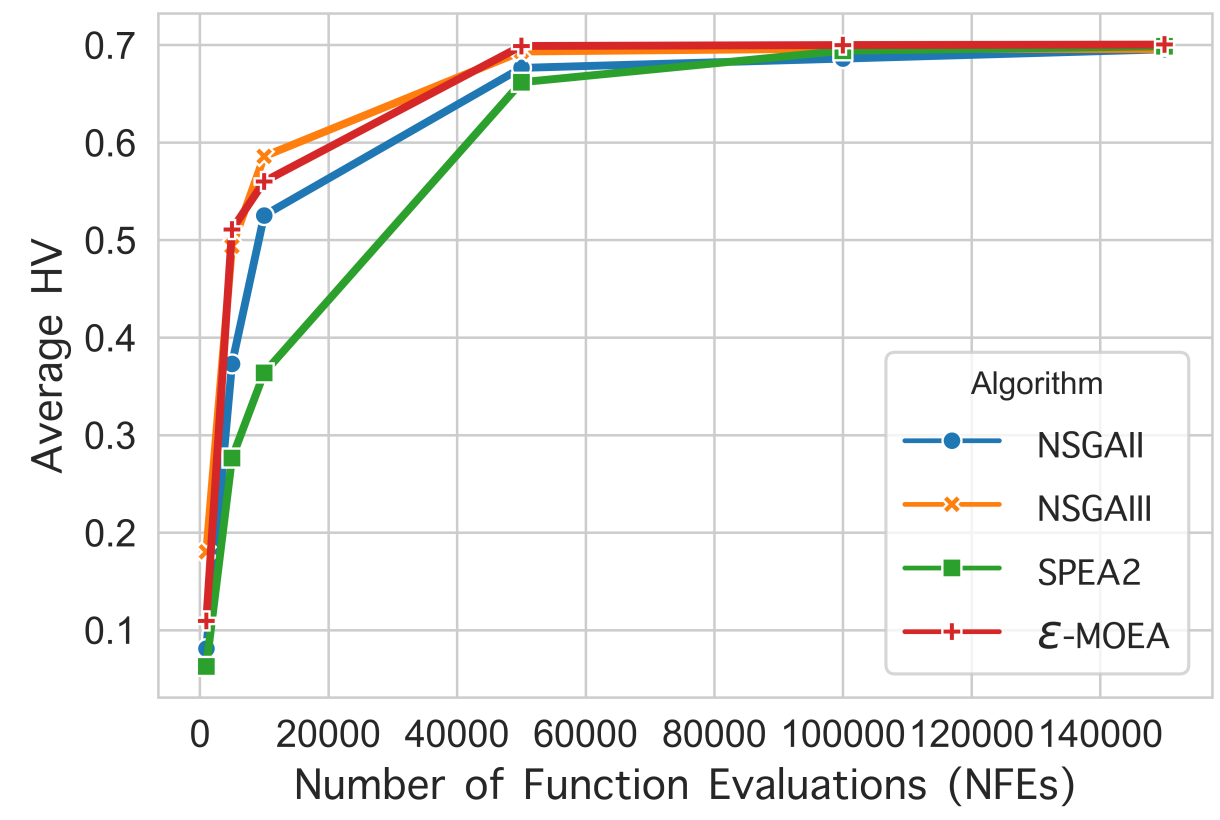

FiguRE 7.10: The Number of Function Evaluations (NFEs) vs. average HV for NSGAII, NSGAIII, SPEA2, and $\varepsilon$-MOEA. 
Chapter 7. Personalized Resource Allocation in Wireless Networks: An AI-Enabled and BD-Driven Multi-Objective Optimization

the considered MOEAs. In Fig. 7.9, it can be observed that HV decreases as the number of users increases. Accordingly, conclude the following: as the complexity of the problem increases, the quality of the output solutions decreases for a fixed amount of computing resources.

\subsubsection{The impact of the required solution quality on complexity}

In order to further improve the quality of the solutions as the complexity of the problem increases, MOEAs need a higher number of evaluations for each run; hence, more computing resources are required. In order to investigate this assumption, using a random instance, the average HV with varying NFEs is computed. In this experiment, the number of network users is fixed at 6 users. Fig. 7.10 compares the average HV vs. NFEs for the considered MOEAs. As anticipated, the HV values rise as the NFEs increase. After a certain NFEs limit, the average HV stagnates. In practice, the network should be able to decide the optimum NFEs in order to optimize the utilization of computing resources and make the computations more efficient. Usually, the optimum NFEs depends on several factors, including the network environment, the number of users, and the selected MOEA algorithm. 


\section{Chapter 8}

\section{Preserving User Privacy in Personalized Wireless Networks}

Personalized wireless networks are designed to track user satisfaction levels using $M L$ models that are trained and updated using context data collected from users. Nonetheless, user data collection and transmission make sensitive user information prone to various privacy attacks. Therefore, in the chapter, a privacy-preserving framework is proposed for personalized wireless networks. The proposed framework is enabled by Differential Privacy (DF), which is an influential privacy technology that offers a rigorous and provable privacy guarantee. Besides, the proposed framework is supported by a multi-stage prediction process, which adds a second layer of privacy protection to raw user data. Lastly, the chapter investigates the impact of privacy budget and data size on the quality of the user satisfaction predictors in personalized wireless networks. 
The pervasive increase in the number of smart devices and the emerging internetof-things (IoT) sensors have made user data ubiquitous. As a result, numerous types of applications surface every year with which value is created by analyzing user data. An example of such applications is personalized wireless networks. The main premise of wireless network personalization is to allocate network resources in a way that achieves the required personalized user satisfaction level for each user using the minimum amount of resources. In practical terms, network personalization is the solution to creating the ultimate balance between saving resources and maintaining the required user satisfaction levels in wireless networks. To realize the promised benefits of wireless network personalization, user satisfaction levels should be tracked, analyzed, and predicted in real-time. User satisfaction tracking in personalized networks is enabled by Machine Learning (ML) models deployed to predict user satisfaction in real-time based on user context-behavioral data. Then, the user satisfaction prediction model is used to solve a computationally expensive optimization problem, which is, therefore, done in a scalable cloud infrastructure. To build and continuously update a predictive model for user satisfaction, and to perform the prediction process, user data should continuously be collected and transmitted to the cloud. Yet, having access to user personal data is difficult and extremely controversial. As a matter of fact, one of the main barriers that are holding back research in personalized wireless networks, despite its tremendous benefits to both users and service providers, is the lack of publically available user behavioral and satisfaction data in wireless networks due to privacy and confidentiality concerns. To overcome this issue, and to stimulate research and development in network personalization, in Chapter 4, user datasets with behavioral context information along with the associated satisfaction levels were modeled and synthesized. The proposed datasets are consolidated with realistic characteristics to accurately mimic user satisfaction behavior in personalized wireless networks. However, as we move towards practical implementation and deployment of this technology, privacy-preserving solutions should be adopted and integrated to gain the benefits of personalization without compromising users' privacy and their sensitive data.

Primarily, user privacy is realized by data anonymization [124]. The main idea behind this method revolves around encrypting/removing any type of personally identifiable data. Also, obfuscation is a type of data sanitization through which user private information is converted into inaccurate information, making it difficult to understand and analyze [125]. Nevertheless, several research articles proved the fragility of these methods as cross-referencing techniques with public datasets 
could enable the identification of the people associated with the anonymized data [126]. One alternative to data anonymization, which is one of the most commonlyaccepted solutions to provide provable protection against privacy leaks is Differential Privacy (DP) [125]. DP is a framework designed to share information describing the patterns within data instead of sharing data itself, which is done by randomly perturbing the original data records in order to prevent the adversary from identifying whether a data record is arbitrary or not. One of the key advantages of DP is the fact that it is built using a statistical model with which the risk of privacy leaks can be quantified. However, the protection of user privacy using DP comes at a cost. DP requires big amounts of data and it decreases the utility of data due to the added noise.

In this chapter, since user data is a foundational aspect of personalizing wireless networks, a framework is proposed to integrate DP in wireless networks to realize network personalization without compromising users' private information. The proposed framework comprises two main stages. The first stage is to integrate DP into the pre-processing step in order to sanitize user personal data prior to transmitting it to the cloud. The second stage integrates DP into the trained user satisfaction ML model in order to protect the models against privacy leaks. Also, to further protect user private data, the proposed framework suggests training the ML model at the user edge and splitting the prediction process into two parts, where the first part is performed at the user edge using secret weights that never leave the user device and the second part is performed in the cloud. To implement and test the proposed framework, Deep Neural Networks (DNN) are utilized due to their adaptability and robustness. Finally, the impact of the privacy budget and data size on the level of privacy provided by the framework is studied.

\subsection{Differential Privacy}

Differential privacy [127] is one of the most prominent frameworks designed to share information about datasets without compromising and disclosing private and sensitive information about individual users. In this section, a brief background on DP is provided and the two main types of DP are discussed.

\subsubsection{Definition of differential privacy}

DP is defined in terms of the concept of adjacent databases, which usually depends on the application. For instance, training datasets in personalized wireless 
networks are a set of user context vectors labeled with user satisfaction levels in wireless networks. Two context-user satisfaction datasets are adjacent if they differ in a single entry. Based on the concept of adjacent datasets, the formal definition of DP is as follows:

Definition 8.1.1. A randomized function $\mathcal{F}: \mathcal{D} \rightarrow \mathcal{R}$ with domain $\mathcal{D}$ and range $\mathcal{R}$ satisfies $(\epsilon, \delta)$-differential privacy if any two adjacent inputs $d, d^{\prime} \in \mathcal{D}$ and for any subset of outputs $\mathcal{X} \subseteq \mathcal{R}$ it holds that

$$
\operatorname{Pr}[\mathcal{F}(d) \in \mathcal{X}] \leq e^{\epsilon} \operatorname{Pr}\left[\mathcal{F}\left(d^{\prime}\right) \in \mathcal{X}\right]+\delta
$$

That is, even if adversaries have the entire $\mathcal{D}$ except for a single entry, they can not infer about that entry from the output of the function/algorithm $\mathcal{F}$. $\delta$ is a variable representing the possibility that DP is broken with a probability. $\epsilon$ is considered a metric for privacy loss, where a smaller $\epsilon$ makes $\mathcal{F}$ more privacypreserving with less privacy loss. The key ingredient to DP functions/algorithms is noise, which provides the necessary randomness to protect sensitive user data. In other words, a vector valued function $\mathcal{F}$ with sensitivity $\mathcal{S}(\mathcal{F})$ can be made $\epsilon$-differentially private by adding an independent Laplace noise vector $n$, where

$$
\operatorname{Pr}[n] \propto e^{-\frac{\epsilon}{\mathcal{S}(\mathcal{F})}\|n\|_{1}}
$$

where $\|.\|_{1}$ is the $L_{1}$ norm [128]. The global sensitivity $\mathcal{S}(\mathcal{F})$ is defined as

$$
\mathcal{S}(\mathcal{F})=\max _{d, d^{\prime}}\left\|\mathcal{F}(\mathrm{d})-\mathcal{F}\left(\mathrm{d}^{\prime}\right)\right\|_{1}
$$

where $d$ and $d^{\prime}$ are neighbor databases. In essence, the definition in this section provides a quantitative idea of differentially private algorithms and privacy loss on a database.

\subsubsection{Central vs. local differential privacy}

The location where noise is added distinguishes two types of DP algorithms and functions, central and local DP. In central DP, data is collected by a trusted entity, which decides and manages the DP analysis performed on the data. The noise in central DP is added in the cloud, where the aggregated data from users is stored. On the other hand, local DP models apply DP analysis and add noise at the user edge after which the output private data is disclosed to the cloud for further analysis. There are some pros and cons associated with both schemes. One 
obvious advantage of local DP is the level of privacy it provides as the original user data is only accessed by the user him $\backslash$ herself. Nonetheless, this high level of protection comes at a cost. Although central DP is not as private as local DP, it requires less amount of noise to ensure privacy, and hence, more accurate predictions. As a result, local DP models require significantly big amounts of data to compensate for the added noise and produce more accurate prediction results. Recently, Google proposed in [129] a new DP design that acts as a middle ground between the two options. Nevertheless, local DP is employed to illustrate how privacy can be preserved in personalized wireless networks.

\subsection{DP-based Privacy-Preserving Framework for Personalized Wireless Networks}

In order to achieve autonomous/non-intrusive personalized wireless networks, where direct user feedback is not required, wireless networks need to have predictive capabilities of user satisfaction in various contexts. In order to build these predictive models in the cloud, wireless networks should have access to user data, which poses a fundamental threat to users' private information. For this reason, in this section, a DP-based framework is proposed through which how personalized wireless networks can optimize network services to the user level and preserve privacy, simultaneously, is illustrated. As shown in Fig. 8.1, the proposed privacy-preserving framework consists of two main stages, training private user satisfaction ML models locally and using the trained ML models to predict the personalized user satisfaction levels in the cloud. The proposed framework consists of the following stages:

\subsubsection{Training at the user edge}

1) Aggregate user context data- The first step is to aggregate enough raw user context data. Examples of context data include location, time, and speed.

2) Apply DP-PCA- Principal Component Analysis (PCA) is a useful method for reducing the inherent noise in the data and capturing the main features. Although the output of PCA is dependent on the input raw data, the PCA algorithm disseminates the data and reduce its dimensionality; hence, data disclosed to the cloud is not the actual raw user data. In order to improve the overall privacy and reduce the probability of data leakage, adding noise to PCA by implementing the Differentially Private PCA (DP-PCA) (as described in [130]) is proposed. 


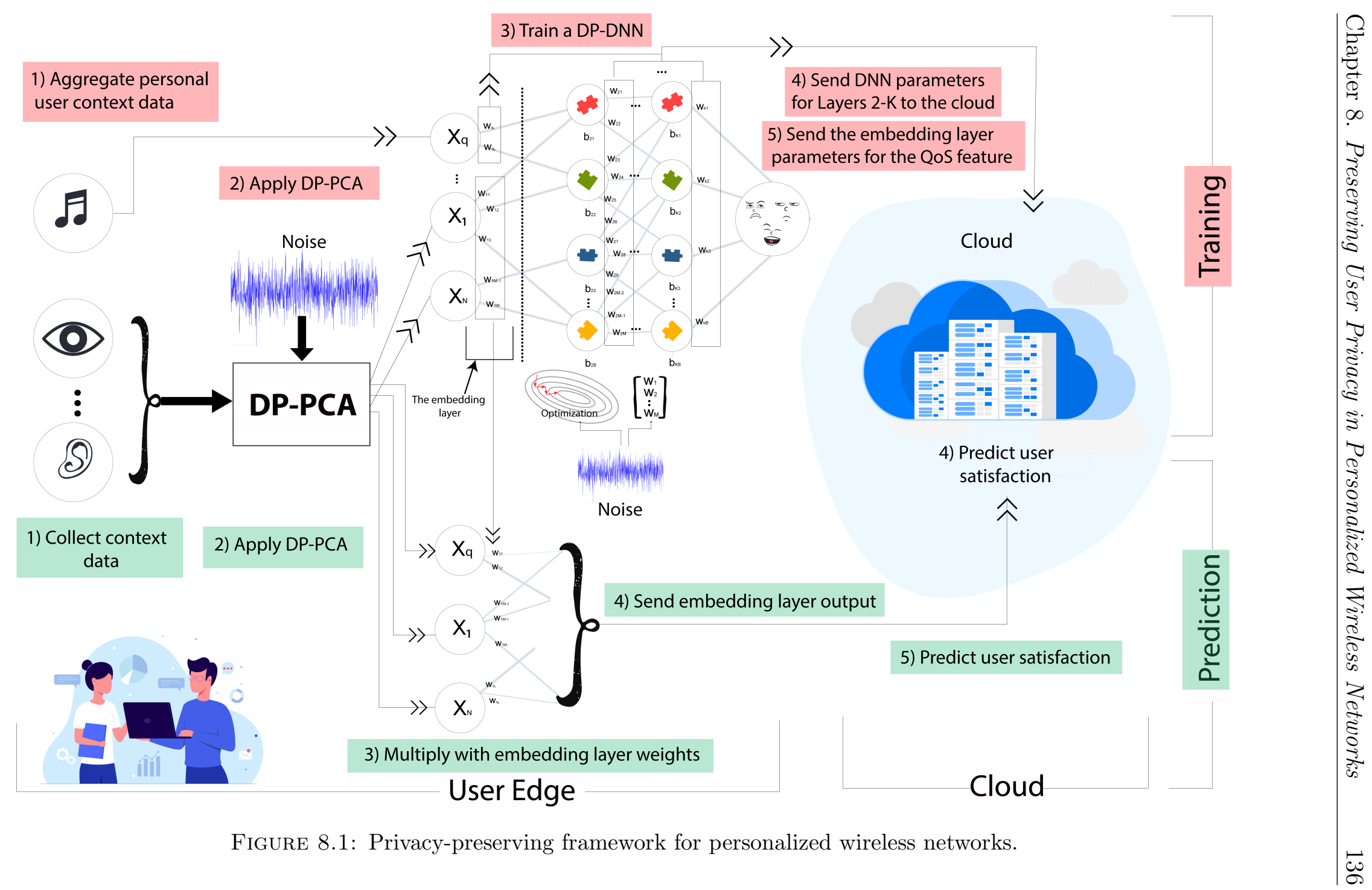


3) Train a DP-DNN- Deep Learning (DL) algorithms leak private information, especially when trained on highly sensitive data. Therefore, even with low privacy budgets, implementing DP is essential to reduce the risk of recovering personal user data. In this step, the proposed framework suggests injecting noise to the weights or the gradient in the optimization process in order to train a DP DNN model at the user edge. For the purpose of this chapter, noise is injected into the gradient during training, as described in [131].

4) Send the parameters of layers 2-K- In this step, as shown in Fig. 8.1, the trained K-layers DNN is clipped after the embedding layer. Also, the weights of the embedding layer are kept at the user edge to act as a latent representation of the raw data. On the other hand, the parameters associated with the remaining layers of the trained DNN model are disclosed to the cloud in order to enable the personalized network to predict user satisfaction and optimizing the Quality-ofService (QoS) based on user context in real-time.

5) Send the embedding layer parameters associated with the QoS feature- The proposed personalized network optimizes user satisfaction at a specific context through several evaluations of user satisfaction levels for different QoS levels. In order to enable the network to perform the optimization process described in Chapter 7 in the cloud, the network should have access to the weights associated with the raw QoS feature (i.e., without PCA).

\subsubsection{Predict user satisfaction in the cloud}

1) Collect context data- The first step is to collect user context at which the personalized network optimizes and predict user satisfaction.

2) Apply DP-PCA- The second step is to transform the raw data vector using the DP-PCA trained in the training stage.

3) Multiply with the embedding layer weights- In this step, the DP-PCA output is multiplied by the embedding layer weights computed in the training process. These weights never leave the user edge, and hence act as embeddings to further burry correlations in the raw private and sensitive user data.

4) Send the embedding layer output- In this step, the output of the embedding layer is disclosed to the cloud, which acts as input to the second part of the DNN model.

5) Predict user satisfaction- Use the output of the embedding layer as an input to Layer 2 in order to predict user satisfaction in real-time using the trained ML model. 


\subsection{Empirical Analysis and Discussions}

In this section, the proposed framework is simulated in order to investigate the effect of utilizing DP and the proposed multi-stage prediction process on the performance of the trained user satisfaction predictor. Moreover, the effect of the privacy budget $\epsilon$ and data size on the performance of the predictor is examined.

\subsubsection{Dataset and simulation setup}

The proposed framework in Fig. 8.1 is simulated using Python and the DNN is simulated using TensorFlow [132]. The dataset utilized in this chapter consists of 200,000 records and it is described in Chapter 4. Also, the dataset can be found in a publicly available GitHub repository [39]. Before training, data is normalized and balanced using the SMOT algorithm. After pre-processing, the dataset consists of 70 feature columns. Also, the values of user satisfaction labels range between 1 and 5 and the number of PCA components is set to 50 .

\subsubsection{Experiment 1: The impact of differential privacy on the quality of the user satisfaction predictor}

This section presents an investigation on the effect of integrating DP on the performance of the proposed framework. Fig. 8.2 depicts the cross-validation accuracy for the following predictors: (I) DNN with a multi-stage prediction process (DNN), (II) PCA followed by DNN, (III) DP-PCA followed by DNN, (IV) PCA followed by a DP-DNN, and (V) DP-PCA followed by DP-DNN. The privacy budget $\epsilon$ is set to 5 for DP-PCA and to $10^{7}$ for DP-DNN. Besides, $\delta$ is set to $10^{-5}$ for all DP algorithms. As shown in Fig. 8.2, DNN, PCA+DNN, and DP-PCA+DNN achieved similar average accuracies (around 90\%). Nonetheless, the variance for DP-PCA $+\mathrm{DNN}$ is higher $( \pm 0.42 \%)$ compared to $\mathrm{PCA}+\mathrm{DNN}$ $( \pm 0.39 \%)$ and DNN $( \pm 0.32 \%)$. The variance increase for PCA is due to the loss of information, which also affects DP-PCA. On the other hand, due to the noise injected into the DP-DNN, PCA+DP-DNN and PCA+DP-DNN achieved lower average accuracies (around $72 \%$ ). It can be observed from Fig. 8.2 that the multistage prediction DNN achieved the best performance, which is expected due to the fact that the multi-stage process does not interfere with the actual training and prediction processes. Besides, it can be deduced that applying DP to DNN considerably decreases the performance by an amount dependent on the chosen 


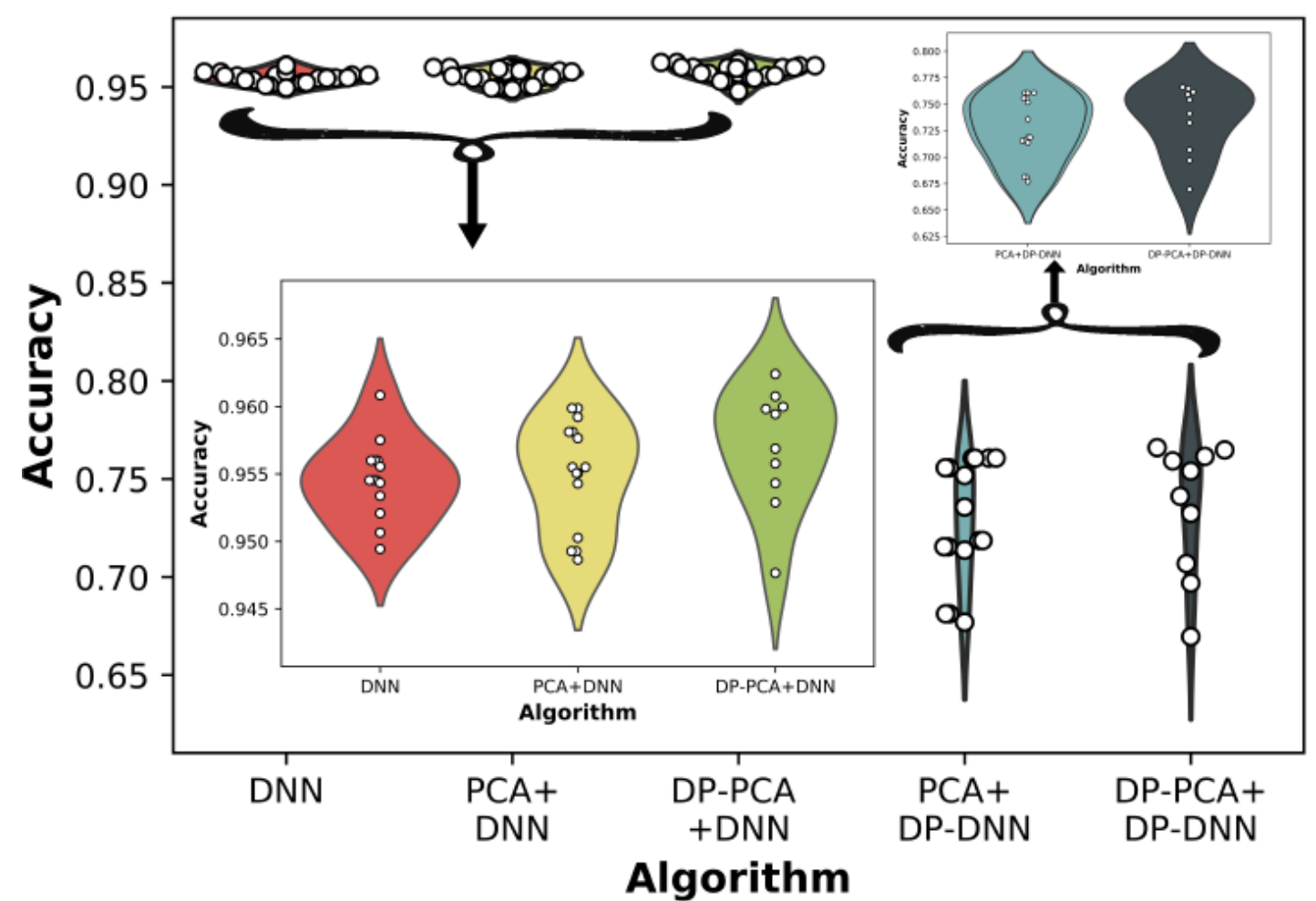

FiguRE 8.2: 10-folds cross-validation swarm plot for (I) DNN, (II) PCA+DNN, (III) DP-PCA+DNN, (IV) PCA+DP-DNN, and (V) DP-PCA+DP-DNN.

privacy budget. It is also worth mentioning that, although DP-PCA affects robustness (i.e., variance), for the chosen number of PCA components, the simulated DP-PCA has less impact on accuracy as compared to DP-DNN.

\subsubsection{Experiment 2: The effect of privacy budget and train- ing data size on performance}

As mentioned earlier, the smaller the $\epsilon$, the higher the amount of noise added, and thereby the higher the protection the DP function/algorithm provides. Fig. 8.3 depicts the accuracy of DP-DNN for different levels of privacy budget $\epsilon$. It can be observed from Fig. 8.3 that, although the accuracy values fluctuate due to the added noise, the overall accuracy increase as $\epsilon$ increase, which is expected as higher $\epsilon$ values indicate that less noise is being injected into the algorithm. Nevertheless, lower $\epsilon$ values are preferable as they provide higher privacy protection. Therefore, to improve accuracy without sacrificing privacy protection (i.e., increase $\epsilon$ without decreasing accuracy), the model should be trained with a bigger amount of data. Fig. 8.3 compares the performance of two DP-DNNs using $50 \%$ (100,000 records) 


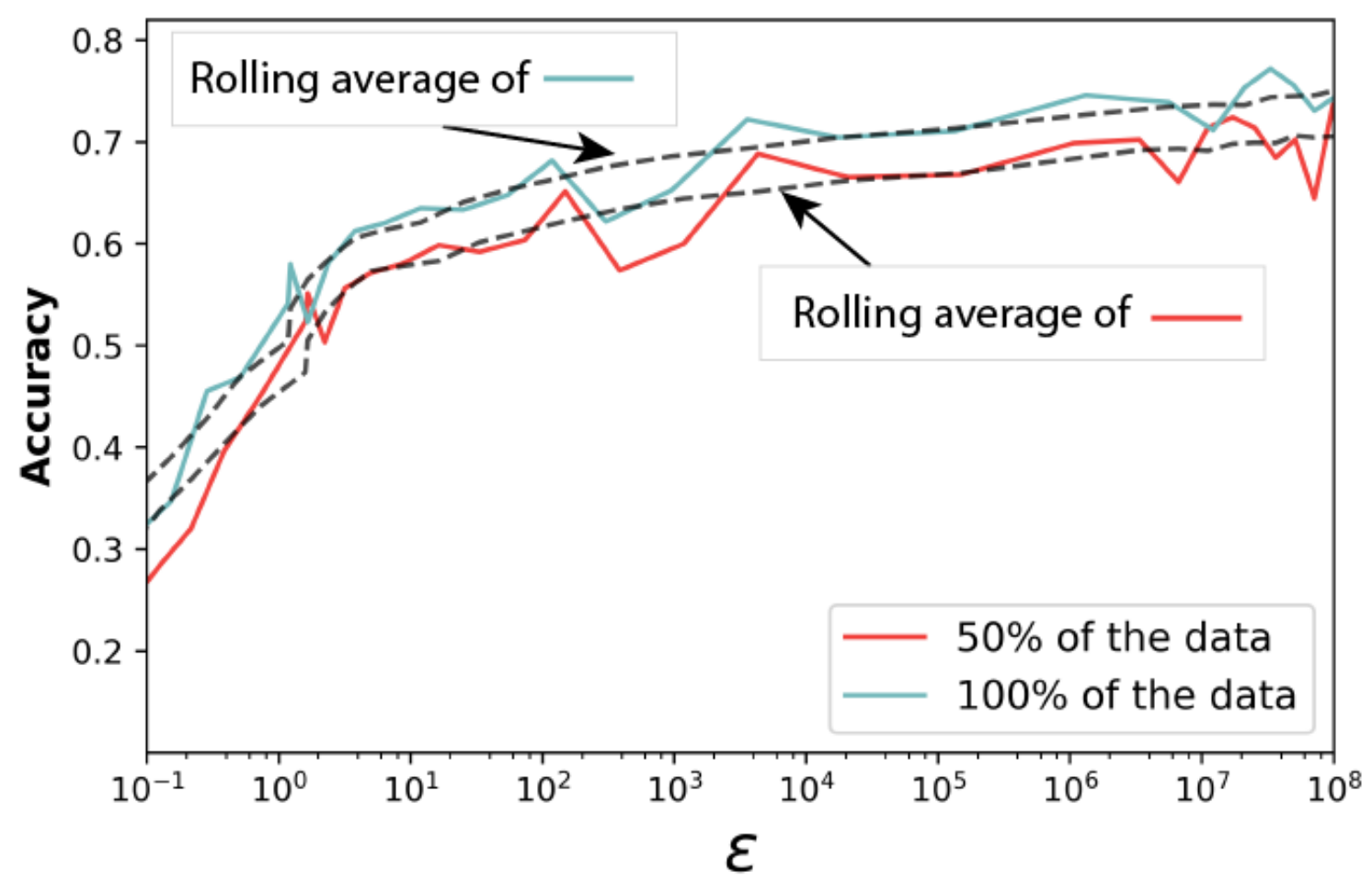

Figure 8.3: Accuracy of DP-DNN vs. privacy budgets $\epsilon$ for two different data sizes.

and 100\% (200,000 records) of the data. As shown in Fig. 8.3, the DP-DNN trained with $100 \%$ of the data achieved higher accuracy values for the same privacy budget $\epsilon$ compared to the DP-DNN trained with $50 \%$ of the data. As a result, it can be deduced that feeding the DP satisfaction predictor with more data will improve the performance without sacrificing the required privacy budget. 


\section{Chapter 9}

\section{Conclusions and Future Research Directions}

This chapter summarizes the contributions presented in this thesis. Besides, the chapter presents different alternatives and options where the proposed technology could be applicable and has value. Finally, some open research issues for future research projects are discussed. 


\subsection{Synopsis of the Proposed Technology}

This thesis introduced a method of personalized resource management for wireless network resources. The proposed personalized networks framework is considered as an improvement on $5 \mathrm{G}$ networks comprising a layer for micro-managing the resources within a slice, tailoring them to the end user's specific needs and requirements, resulting in greater agility and flexibility. The technology is summarized as follows:

- The proposed personalized resource management method comprises the following:

- Establishing a Zone of Tolerance (ZoT) model for user satisfaction, where the range of QoS on a wireless network is divided into a number of discrete QoS levels.

- Responding to a user request for network resources, by:

* Identifying context and persona associated with the user request.

* Determining a predicted ZoT level based on the identified context and persona.

* Identifying the QoS range necessary to reach the targeted satisfaction level for the user request.

* Identifying the lowest QoS required to provide a service within the predicted ZoT level.

* Satisfying the user request by allocating network resources based on the identified lowest QoS level required to achieve the targeted satisfaction.

- Mapper function is proposed to model and implement the ZoT model and to identify user satisfaction behavior from context features and persona type.

- To model the effect of missing context variables on the performance of the user satisfaction prediction process, noise is integrated into the satisfaction mapper.

- The proposed technology utilizes QoS as a measure for ZoT ranges, which comprises at least one metric selected from the group consisting of data rate, reliability, latency, jitter, etc. 
- Context data comprises one or more of the parameters: time, day, location, speed, activity, service request arrival, application choice, application service choice, request rate demand, given rate, and $\Delta$ (the difference between the desired service and offered service). Activity and other immeasurable context features are predicted using Machine Learning (ML) from data collected using user sensors.

- The proposed personalization framework predicts user satisfaction levels based on context data and persona type; hence datasets are gathered from the network.

- Since datasets of user behavior data and the associated user satisfaction levels are not available yet, a synthetic user dataset design is proposed to model, test, and study personalized wireless network.

- In the proposed dataset design, context features are organized into one or multiple tree structures, each of which being directed to a set of users with similar behavioral patterns or personas.

- The problem of real-time user satisfaction tracking and prediction is approached through building a multi-phase ML model to predict user satisfaction levels for each user, where the machine learning model utilizes the extracted persona information by a persona ML model.

- The first phase in the multiphase user satisfaction prediction and tracking is a persona ML model, whereas the second phase is a user satisfaction ML model.

- A user persona prediction framework is proposed to associate users with predefined personas, comprising:

- Quantifying and formatting collected data before feeding it to the ML algorithm in order to achieve better prediction results.

- Dividing data into stacks to decide based on a series of data records instead of one instance.

- Compute the confidence of each persona label and pick the persona label with a higher confidence value.

- A satisfaction real-time data collection and prediction framework to label context data with ground truth satisfaction levels is proposed, which comprises: 
- Acquiring context data via monitoring sensors including microphones and cameras.

- Aggregating and analyzing collected data and predicting missing and future context information.

- Associating the acquired context data with user satisfaction values.

- Measuring the correlation between user satisfaction values and the service being used by the user.

- The labeled dataset by the network is used to train an ML model to predict user satisfaction value from context data.

- User satisfaction level is continuously measured and compared with the predicted values by the ML model. If the prediction result is correct, the data sample is feedback to the ML model to continuously train it in real-time and improve the performance as more data samples become available. In contrast, if the result is not correct, the network penalizes the ML model in a process called concept drift.

- A deep neural network structure is proposed to use context data for predicting future user satisfaction values.

- A method of personalized resource scheduling for wireless network resources is proposed, where the resource allocation method comprises solving a MultiObjective Optimization (MOO) problem.

- The performed multi-objective optimization is designed to ingest real-time data and use the trained ML models to determine the minimum amount of resources required to achieve the targeted satisfaction level.

- Allocating network resources further comprises allocating network resources as a multi-objective decision, based on optimizing resources and user satisfaction.

- Allocating network resources further comprises allocating network resources as a Pareto-based multi-objective solution.

- The proposed resource allocation MOO problem is solved using evolutionary multi-objective optimization (EMOO). 
- The performance of the EMOO algorithms is assessed using the following performance metrics: Non-dominated Vector Generation Ratio (NGR), Generational Distance (GD), Spacing (SP), Hypervolume (HV), and Inverted Generational Distance (IGD).

- The non-dominated Pareto front reference solution set is extracted from multiple sets generated by different EMOO algorithms.

- Statistical analysis is conducted on the solutions produced by different EMOO algorithms in order to decide which algorithm significantly performed better.

- The impact of uncertainty introduced by the satisfaction prediction ML model on the performance of the EMOO algorithms is investigated.

- The impact of the number of network users and the required solution quality on the complexity of the MOO problem is investigated.

- A surrogate management scheme is proposed to track and verify the predicted user satisfaction in personalized wireless networks and to prevent the divergence of the optimization process from the actual user satisfaction levels.

- A privacy-preserving framework that is based on the concept of Differential Privacy (DF) is proposed to protect user privacy in personalized wireless networks, comprising:

- A multi-stage prediction process to further protect user data in personalized wireless networks, comprising:

* Training private user satisfaction ML models locally.

* Using the trained ML models to predict the personalized user satisfaction levels in the cloud.

- Adding a Differentially Private Principle Component Analysis (DPPCA) layer to process raw personal user data.

- Using an embedding layer to further burry the correlations within the raw user data.

- Keeping the parameters of the embedding layer on the user device to act as a second protection layer for user privacy.

- Using a Diffeernetially Private Deep Neural Network (DP-DNN) to train the user satisfaction ML model locally. 
- The effect of the privacy budget $\epsilon$ and data size on the performance of the predictor is examined.

\subsection{Additional Options and Applications}

A number of variations and modifications can be made without departing from the scope of the presented technology. While the presented technology focuses on the two primary objectives, i) maximize average user satisfaction and ii) minimize the allocated resources by the network, the implementation of the system may include the following add-ons:

- Automate hyperparameter tuning and machine learning model selection.

- Augment and semi-synthesize a big data dataset with many users and user personas.

- Develop a distributed parallelized version of the algorithms and optimizers.

- Use customized heuristic and metaheuristics algorithms to solve the formulated multi-objective problem.

- Use a different satisfaction mapper function to replace the stair function.

- Modeling satisfaction as a continuous value.

- Use different ML design models to achieve persona and user satisfaction prediction.

- Adding different context features to the dataset design. The more features, the more accurate results are achieved by the ML models.

- Using different multi-objective formulations to achieve the same goal. The goal of the multi-objective optimizer is to provide service providers with a Pareto-front solution. This solution trades off satisfaction with the amount of recourse required. The optimizer provides service providers with the flexibility to choose a personalized user satisfaction target for each user and find the minimum amount of resources to achieve this target.

The details of the technology proposed in this thesis need not be limited to wireless networks or even to communication networks. The framework and functionality of extracting and/or predicting context and user satisfaction in an unintrusive way, 
in real-time, can be applied in a broad range of applications. At the very least, these applications would include connected and autonomous vehicles, battlefield (tactical) scenarios, gaming and wearables (AV/RV, haptics, etc.) In fact, the proposed technology can be applied to any context in which application delivery can be fine-tuned in real-time based on a number of factors including the end user's likes/dislikes, ambiance, circumstances, mood, how much she/he is willing to pay, etc.

\subsection{Open Research Issues}

While the proposed personalization framework has the potentials to achieve the ultimate balance between the conservation of resources and increasing user satisfaction, there are a number of critical open directions that are yet to be explored prior to practical implementation. This section summarizes and lists some of these directions and open problems.

\subsubsection{Dynamic users data}

User data is highly dynamic and unpredictable. Therefore, the process of data acquisition and preprocessing is extremely challenging and necessitates more investigation and exploration.

\subsubsection{Non-intrusive user feedback}

As mentioned earlier, there are efforts in the literature to sense and predict user emotions and opinions in a non-intrusive manner using various sensors data. However, the process of correlating the predicted feelings to user satisfaction levels in wireless networks constitutes a challenging yet interesting line of research.

\subsubsection{User persona clustering}

In the early stages of adopting wireless network personalization, networks do not have enough information about the types of personas and shared behaviors among certain groups of users. Therefore, unsupervised clustering for user personas is an area of research that needs more study and analysis. 


\subsubsection{An effective and efficient surrogate management scheme}

In this thesis, a surrogate management scheme is proposed in order to prevent the convergence of erroneous Pareto front solutions. Nonetheless, the prototype implemented in this thesis did not employ a surrogate management scheme; hence, the simulated surrogate-assisted personalized network was not able to maintain the required satisfaction level. Therefore, a more thorough study and analysis of the surrogate management scheme should be performed. Interestingly, the simulation results showed similar trends between the surrogate assisted (SPN-estimated) and direct feedback (SPN-feedback) personalized networks. Yet, the SPN-estimated had a negative bias in the satisfaction results and a positive bias in the saved resources results. For this reason, further analysis of the relationship between SPNestimated and SPN-feedback is necessary. Furthermore, the simulation results presented in this thesis raised a vital question about the efficacy of adding a fixed bias to the outcome of surrogate-assisted personalized wireless networks in order to resolve the divergence issues and whether this bias can be calculated and predicted in real-time.

\subsubsection{Accurate prediction of user satisfaction}

Among the main pillars of personalized wireless networks' design is the accurate prediction of user satisfaction levels. Therefore, further investigation into methods and advanced ML structures that can improve the accuracy of predicting user satisfaction in real-time is required. Besides, while the synthetic dataset utilized in this article provided valuable insights into the dynamics of personalized wireless networks, real-world user context and satisfaction data is fundamental to verify the developed models and framework.

\subsubsection{Advaned IEC MOEAs}

As mentioned earlier, IEC MOEAs proposed in the literature are designed to incorporate user feedback into the optimization process. Leveraging IEC optimization in personalized networks could reveal drastic improvements in optimization performance and complexity reduction compared to traditional MOEAs. 


\subsubsection{Optimizing other aspects of wireless networks}

This thesis highlighted resource scheduling, which is one of several aspects of wireless networks that can benefit from the proposed personalization scheme. Examples of such include network failure detection and network security decisions. Additionally, although the proposed OPA problem is formulated for cellular networks, it can be formulated and redesigned for any network with users, such as WiFi and wired networks. Finally, the main ideas and the premise of the thesis can be applied to any application that requires user feedback.

\subsubsection{Data collection, processing, and transmission over- head}

Although personalized wireless networks are envisioned to achieve the ultimate balance between resource-saving and maintaining the required user satisfaction, personalization requires data collection and transmission of user satisfaction and context information from the user to the network, which will result in extra overhead cost. Therefore, it is crucial to investigate and model the cost associated with network personalization in terms of processing power, transmission overhead and delay.

\subsubsection{The correlation of detected user emotions with the provided service quality}

As illustrated in Chapter 6, the process of collecting and capturing the actual user satisfaction information involves user emotion detection and correlating them to the provided QoS. Detecting emotions using various sensor data has been tackled by researchers in the literature. Nonetheless, the correlation process needs to be investigated and studied. An example question that the network should be able to answer is the following: are the detected low satisfaction emotions due to the network failing to provide the requested QoS, or because the user read an infuriating post on Facebook. 


\section{Bibliography}

[1] A. Ericsson, "Ericsson mobility report," Ericsson, Sweden, Tech. Rep. EAB19:003442 Uen, June 2019.

[2] A. Parasuraman, V. A. Zeithaml, and L. L. Berry, "A conceptual model of service quality and its implications for future research," the Journal of Marketing, pp. 41-50, 1985.

[3] H. Zhang, H. Liu, J. Cheng, and V. C. M. Leung, "Downlink energy efficiency of power allocation and wireless backhaul bandwidth allocation in heterogeneous small cell networks," IEEE Transactions on Communications, vol. 66, no. 4, pp. 1705-1716, April 2018.

[4] H. Lim and T. Hwang, "User-centric energy efficiency optimization for MISO wireless powered communications," IEEE Transactions on Wireless Communications, vol. 18, no. 2, pp. 864-878, February 2019.

[5] O. L. Alcaraz Lopez, H. Alves, and M. Latva-aho, "Joint power control and rate allocation enabling ultra-reliability and energy efficiency in SIMO wireless networks," IEEE Transactions on Communications, vol. 67, no. 8, pp. 5768-5782, August 2019.

[6] A. Asheralieva and Y. Miyanaga, "An autonomous learning-based algorithm for joint channel and power level selection by D2D pairs in heterogeneous cellular networks," IEEE transactions on communications, vol. 64, no. 9, pp. 3996-4012, 2016.

[7] X. Wang, T. Jin, L. Hu, and Z. Qian, "Energy-efficient power allocation and Q-learning-based relay selection for relay-aided D2D communication," IEEE Transactions on Vehicular Technology, vol. 69, no. 6, pp. 6452-6462, 2020.

[8] A. Asheralieva, "Bayesian reinforcement learning-based coalition formation for distributed resource sharing by device-to-device users in heterogeneous 
cellular networks," IEEE Transactions on Wireless Communications, vol. 16, no. 8, pp. 5016-5032, 2017.

[9] M. I. Khan, M. M. Alam, Y. Le Moullec, and E. Yaacoub, "Cooperative reinforcement learning for adaptive power allocation in device-to-device communication," in 2018 IEEE 4th World Forum on Internet of Things (WF-IoT), 2018, pp. 476-481.

[10] I. Budhiraja, N. Kumar, and S. Tyagi, "Deep reinforcement learning based proportional fair scheduling control scheme for underlay D2D communication," IEEE Internet of Things Journal, pp. 1-1, 2020.

[11] Y.-J. Liu, S.-M. Cheng, and Y.-L. Hsueh, "eNB selection for machine type communications using reinforcement learning based Markov decision process," IEEE Transactions on Vehicular Technology, vol. 66, no. 12, pp. $11330-11338,2017$.

[12] X. He, K. Wang, H. Huang, T. Miyazaki, Y. Wang, and S. Guo, "Green resource allocation based on deep reinforcement learning in content-centric iot," IEEE Transactions on Emerging Topics in Computing, 2018.

[13] L. Nie, Z. Ning, M. Obaidat, B. Sadoun, H. Wang, S. Li, L. Guo, and G. Wang, "A reinforcement learning-based network traffic prediction mechanism in intelligent internet of things," IEEE Transactions on Industrial Informatics, pp. 1-1, 2020.

[14] A. Galindo-Serrano and L. Giupponi, "Distributed Q-learning for interference control in OFDMA-based femtocell networks," in Proc. IEEE 71st Vehicular Technology Conference (VTC 2010-Spring), 2010, pp. 1-5.

[15] M. Dirani and Z. Altman, "A cooperative reinforcement learning approach for inter-cell interference coordination in OFDMA cellular networks," in Proc. 8th IEEE International Symposium on Modeling and Optimization in Mobile, Ad Hoc and Wireless Networks (WiOpt), 2010, pp. 170-176.

[16] A. Feki, V. Capdevielle, and E. Sorsy, "Self-organized resource allocation for LTE pico cells: a reinforcement learning approach," in Proc. IEEE 75th Vehicular Technology Conference (VTC Spring), 2012, pp. 1-5. 
[17] Y. Wei, F. R. Yu, M. Song, and Z. Han, "User scheduling and resource allocation in HetNets with hybrid energy supply: An actor-critic reinforcement learning approach," IEEE Transactions on Wireless Communications, vol. 17, no. 1, pp. 680-692, 2018.

[18] F. Tang, Y. Zhou, and N. Kato, "Deep reinforcement learning for dynamic uplink/downlink resource allocation in high mobility 5G HetNet," IEEE Journal on Selected Areas in Communications, pp. 1-1, 2020.

[19] J. Moysen and L. Giupponi, "A reinforcement learning based solution for self-healing in LTE networks," in Proc. IEEE 80th Vehicular Technology Conference (VTC Fall), 2014, pp. 1-6.

[20] C.-K. Wen, S. Jin, K.-K. Wong, J.-C. Chen, and P. Ting, "Channel estimation for massive mimo using Gaussian-mixture bayesian learning," IEEE Transactions on Wireless Communications, vol. 14, no. 3, pp. 1356-1368, 2015 .

[21] Y.-Y. Liu and S.-J. Yoo, "Dynamic resource allocation using reinforcement learning for LTE-U and WiFi in the unlicensed spectrum," in Proc. IEEE Ninth International Conference on Ubiquitous and Future Networks (ICUFN), 2017, pp. 471-475.

[22] Y. He, Z. Zhang, and Y. Zhang, "A big data deep reinforcement learning approach to next generation green wireless networks," in Proc. 2017 IEEE Global Communications Conference (Globecom), 2017, pp. 1-6.

[23] B. Gu, Y. Wei, X. Liu, M. Song, and Z. Han, "Traffic offloading and power allocation for green HetNets using reinforcement learning method," in 2019 IEEE Global Communications Conference (GLOBECOM), 2019, pp. 1-6.

[24] K. W. Choi and E. Hossain, "Estimation of primary user parameters in cognitive radio systems via hidden Markov model," IEEE Transactions on sSignal Processing, vol. 61, no. 3, pp. 782-795, 2013.

[25] A. Assra, J. Yang, and B. Champagne, "An EM approach for cooperative spectrum sensing in multiantenna CR networks," IEEE Transactions on Vehicular Technology, vol. 65, no. 3, pp. 1229-1243, 2016.

[26] M. A. Qureshi and C. Tekin, "Online bayesian learning for rate selection in millimeter wave cognitive radio networks," in IEEE INFOCOM 2020 IEEE Conference on Computer Communications, 2020, pp. 1449-1458. 
[27] A. Kaloxylos, "A survey and an analysis of network slicing in 5G networks," IEEE Communications Standards Magazine, vol. 2, no. 1, pp. 60-65, March 2018 .

[28] H. Zhang, N. Liu, X. Chu, K. Long, A. Aghvami, and V. C. M. Leung, "Network slicing based $5 \mathrm{G}$ and future mobile networks: Mobility, resource management, and challenges," IEEE Communications Magazine, vol. 55, no. 8, pp. 138-145, August 2017.

[29] I. Afolabi, T. Taleb, K. Samdanis, A. Ksentini, and H. Flinck, "Network slicing and softwarization: A survey on principles, enabling technologies, and solutions," IEEE Communications Surveys Tutorials, vol. 20, no. 3, pp. 2429-2453, 2018.

[30] X. Foukas, G. Patounas, A. Elmokashfi, and M. K. Marina, "Network slicing in 5G: Survey and challenges," IEEE Communications Magazine, vol. 55, no. 5, pp. 94-100, May 2017.

[31] S. Zhang, "An overview of network slicing for 5G," IEEE Wireless Communications, vol. 26, no. 3, pp. 111-117, June 2019.

[32] X. Xu, H. Zhang, X. Dai, Y. Hou, X. Tao, and P. Zhang, "SDN based next generation mobile network with service slicing and trials," China Communications, vol. 11, no. 2, pp. 65-77, February 2014.

[33] J. Ordonez-Lucena, P. Ameigeiras, D. Lopez, J. J. Ramos-Munoz, J. Lorca, and J. Folgueira, "Network slicing for $5 \mathrm{G}$ with SDN/NFV: Concepts, architectures, and challenges," IEEE Communications Magazine, vol. 55, no. 5, pp. 80-87, May 2017.

[34] K. U. R. Laghari and K. Connelly, "Toward total quality of experience: A QoE model in a communication ecosystem," IEEE Communications Magazine, vol. 50, no. 4, 2012.

[35] R. Alkurd, I. Abualhaol, and H. Yanikomeroglu, "A synthetic user behavior dataset design for data-driven AI-based personalized wireless networks," in Proc. 2019 IEEE International Conference on Communications (ICC Workshops), Shanghai, China, May 2019.

[36] — , "Data-driven user persona prediction framework for personalized wireless networks," in Proc. IEEE 92nd Vehicular Technology Conference (VTC2020-Fall), September 2020. 
[37] — , "User satisfaction prediction framework for personalized networks: A deep neural network approach," submitted to 2020 IEEE International Conference on Communications (ICC).

[38] J. Liu, F. Liu, and N. Ansari, "Monitoring and analyzing big traffic data of a large-scale cellular network with hadoop," IEEE Network, vol. 28, no. 4, pp. 32-39, 2014.

[39] R. Alkurd, I. Abualhaol, and H. Yanikomeroglu. A synthetic user behavior dataset design for data-driven AI-based personalized wireless networks: Dataset. [Online]. Available: https://github.com/rawanalkurd/ Personalization-Framework-Datasets, AccessedinJuly2020.

[40] R. Schoenen and H. Yanikomeroglu, "User-in-the-loop: Spatial and temporal demand shaping for sustainable wireless networks," IEEE Communications Magazine, vol. 52, no. 2, pp. 196-203, 2014.

[41] Y. Bao, H. Wu, and X. Liu, "From prediction to action: Improving user experience with data-driven resource allocation," IEEE Journal on Selected Areas in Communications (J-SAC), vol. 14, no. 8, p. 1, 2015.

[42] X. Hu, J. Cheng, M. Zhou, B. Hu, X. Jiang, Y. Guo, K. Bai, and F. Wang, "Emotion-aware cognitive system in multi-channel cognitive radio ad hoc networks," IEEE Communications Magazine, vol. 56, no. 4, pp. 180-187, April 2018.

[43] M. S. Hossain, G. Muhammad, B. Song, M. M. Hassan, A. Alelaiwi, and A. Alamri, "Audio-visual emotion-aware cloud gaming framework," IEEE Transactions on Circuits and Systems for Video Technology, vol. 25, no. 12, pp. 2105-2118, 2015.

[44] M. S. Hossain and G. Muhammad, "Emotion-aware connected healthcare big data towards 5G," IEEE Internet of Things Journal, vol. 5, no. 4, pp. 2399-2406, August 2018.

[45] B. G. Lee, T. W. Chong, B. L. Lee, H. J. Park, Y. N. Kim, and B. Kim, "Wearable mobile-based emotional response-monitoring system for drivers," IEEE Transactions on Human-Machine Systems, vol. 47, no. 5, pp. 636-649, October 2017.

[46] A. Dutka, AMA Handbook for Customer Satisfaction. Ntc Pub Group, 1993. 
[47] P. Christen and A. Pudjijono, "Accurate synthetic generation of realistic personal information-rich," in Proc. 2009 Pacific-Asia Conference on Knowledge Discovery and Data Mining, Bangkok, Thailand, April 2009, pp. 507514.

[48] Q. Lv, Y. Qiao, N. Ansari, J. Liu, and J. Yang, "Big data driven hidden Markov model based individual mobility prediction at points of interest," IEEE Transactions on Vehicular Technology, vol. 66, no. 6, pp. 5204-5216, 2017.

[49] H. Gjoreski, M. Ciliberto, F. J. Ordoñez Morales, D. Roggen, S. Mekki, and S. Valentin, "A versatile annotated dataset for multimodal locomotion analytics with mobile devices." Sensys, 2017.

[50] D. Anguita, A. Ghio, L. Oneto, X. Parra, and J. L. Reyes-Ortiz, "A public domain dataset for human activity recognition using smartphones." in Proc. 2013 European Symposium on Artificial Neural Networks, Computational Intelligence and Machine Learning (ESANN), Bruges, Belgium, April 2013, pp. $437-442$.

[51] R. O. Duda, P. E. Hart, D. G. Stork et al., Pattern Classification. Wiley New York, 1973, vol. 2.

[52] J. Pruitt and T. Adlin, The Persona Lifecycle: Keeping People in Mind Throughout Product Design. Elsevier, 2010.

[53] J. Manoogian III and J. Elvekrog, "Targeting users based on persona data," June 2014, US Patent 8,751,305.

[54] M. Haas and W. H. Kunz, "How to master the challenges of service mass customization: Apersona-based approach," in Handbook of Research in Mass Customization and Personalization. World Scientific, 2010, pp. 603-621.

[55] B. A. Knott, R. R. Bushey, J. M. Martin, and S. Andrews, "Method, software and system for developing interactive call center agent personas," March 2009, US Patent 7,512,545.

[56] J. Li, M. Galley, C. Brockett, G. P. Spithourakis, J. Gao, and B. Dolan, "A persona-based neural conversation model," arXiv preprint arXiv:1603.06155, 2016. 
[57] S. Kujala and M. Kauppinen, "Identifying and selecting users for usercentered design," in Proc. of the Third Nordic Conference on HumanComputer Interaction. ACM, 2004, pp. 297-303.

[58] A. Shahri, M. Hosseini, M. Almaliki, K. Phalp, J. Taylor, and R. Ali, "Engineering software-based motivation: A persona-based approach," in Proc. 2016 IEEE Tenth International Conference on Research Challenges in Information Science (RCIS), June 2016, pp. 1-12.

[59] R. Sinha, "Persona development for information-rich domains," in Proc. CHI'03 Extended Abstracts on Human Factors in Computing Systems. ACM, 2003, pp. 830-831.

[60] F. Tanudjaja and L. Mui, "Persona: a contextualized and personalized web search," in Proc. of the 35th Annual Hawaii International Conference on System Sciences, January 2002, pp. 1232-1240.

[61] J. An, H. Kwak, and B. J. Jansen, "Validating social media data for automatic persona generation," in Proc. 2016 IEEE/ACS 13th International Conference of Computer Systems and Applications (AICCSA), November 2016, pp. 1-6.

[62] N. Tu, X. Dong, P. P. Rau, and T. Zhang, "Using cluster analysis in Persona development," in Proc. 2010 8th International Conference on Supply Chain Management and Information, October 2010, pp. 1-5.

[63] T. Howley, M. G. Madden, M.-L. O'Connell, and A. G. Ryder, "The effect of principal component analysis on machine learning accuracy with high dimensional spectral data," in Proc. of 2005 International Conference on Innovative Techniques and Applications of Artificial Intelligence. Springer, 2005, pp. 209-222.

[64] P. Baldi and K. Hornik, "Neural networks and principal component analysis: Learning from examples without local minima," Neural Networks, vol. 2, no. 1, pp. 53-58, 1989.

[65] P. Reichl, S. Egger, R. Schatz, and A. D'Alconzo, "The logarithmic nature of QoE and the role of the Weber-Fechner law in QoE assessment," in 2010 IEEE International Conference on Communications, May 2010. 
[66] S. Tasaka, "Bayesian hierarchical regression models for QoE estimation and prediction in audiovisual communications," IEEE Transactions on Multimedia, vol. 19, no. 6, pp. 1195-1208, June 2017.

[67] X. Tao, Y. Duan, M. Xu, Z. Meng, and J. Lu, "Learning QoE of mobile video transmission with deep neural network: A data-driven approach," IEEE Journal on Selected Areas in Communications, pp. 1-1, 2019.

[68] Y. Bao, H. Wu, and X. Liu, "From prediction to action: Improving user experience with data-driven resource allocation," IEEE Journal on Selected Areas in Communications, vol. 35, no. 5, pp. 1062-1075, May 2017.

[69] X. Tao, C. Jiang, J. Liu, A. Xiao, Y. Qian, and J. Lu, "QoE driven resource allocation in next generation wireless networks," IEEE Wireless Communications, vol. 26, no. 2, pp. 78-85, April 2019.

[70] V. Vasilev, J. Leguay, S. Paris, L. Maggi, and M. Debbah, "Predicting QoE factors with machine learning," in 2018 IEEE International Conference on Communications (ICC), May 2018, pp. 1-6.

[71] P. Makris, D. N. Skoutas, and C. Skianis, "A survey on context-aware mobile and wireless networking: On networking and computing environments' integration," IEEE Communications Surveys Tutorials, vol. 15, no. 1, pp. 362-386, 2013.

[72] B. Xu, Y. Fu, Y. Jiang, B. Li, and L. Sigal, "Heterogeneous knowledge transfer in video emotion recognition, attribution and summarization," IEEE Transactions on Affective Computing, vol. 9, no. 2, pp. 255-270, April 2018.

[73] C. Wu and W. Liang, "Emotion recognition of affective speech based on multiple classifiers using acoustic-prosodic information and semantic labels," IEEE Transactions on Affective Computing, vol. 2, no. 1, pp. 10-21, January 2011.

[74] K. Wang, N. An, B. N. Li, Y. Zhang, and L. Li, "Speech emotion recognition using fourier parameters," IEEE Transactions on Affective Computing, vol. 6, no. 1, pp. 69-75, January 2015.

[75] N. Fourati and C. Pelachaud, "Perception of emotions and body movement in the emilya database," IEEE Transactions on Affective Computing, vol. 9, no. 1, pp. 90-101, January 2018. 
[76] A. Kleinsmith and N. Bianchi-Berthouze, "Affective body expression perception and recognition: A survey," IEEE Transactions on Affective Computing, vol. 4, no. 1, pp. 15-33, January 2013.

[77] Z. Yang and S. S. Narayanan, "Modeling dynamics of expressive body gestures in dyadic interactions," IEEE Transactions on Affective Computing, vol. 8, no. 3, pp. 369-381, July 2017.

[78] C. Qing, R. Qiao, X. Xu, and Y. Cheng, "Interpretable emotion recognition using EEG signals," IEEE Access, vol. 7, pp. 94 160-94 170, 2019.

[79] R. Subramanian, J. Wache, M. K. Abadi, R. L. Vieriu, S. Winkler, and N. Sebe, "Ascertain: Emotion and personality recognition using commercial sensors," IEEE Transactions on Affective Computing, vol. 9, no. 2, pp. 147160, April 2018.

[80] C. Zhang, P. Patras, and H. Haddadi, "Deep learning in mobile and wireless networking: A survey," IEEE Communications Surveys Tutorials, pp. 1-1, 2019.

[81] M. A. Alsheikh, D. Niyato, S. Lin, H. Tan, and Z. Han, "Mobile big data analytics using deep learning and apache spark," IEEE Network, vol. 30, no. 3, pp. 22-29, May 2016.

[82] M. H. ter Beek, C. MartÂn-Vide, and V. Mitrana, "Synchronized shuffles," Theoretical Computer Science, vol. 341, no. 1, pp. 263 - 275, 2005. [Online]. Available: http://www.sciencedirect.com/science/article/ pii/S0304397505003208

[83] H. M. Nguyen, E. W. Cooper, and K. Kamei, "Borderline over-sampling for imbalanced data classification," in Proc. Fifth International Workshop on Computational Intelligence $\&$ Applications, vol. 2009, no. 1. IEEE SMC Hiroshima Chapter, 2009, pp. 24-29.

[84] J. Duchi, E. Hazan, and Y. Singer, "Adaptive subgradient methods for online learning and stochastic optimization," Journal of Machine Learning Research, vol. 12, pp. 2121-2159, July 2011.

[85] D. P. Kingma and J. Ba, "Adam: A method for stochastic optimization," arXiv preprint arXiv:1412.6980, 2014. 
[86] A. Ponsich, A. L. Jaimes, and C. A. C. Coello, "A survey on multiobjective evolutionary algorithms for the solution of the portfolio optimization problem and other finance and economics applications," IEEE Transactions on Evolutionary Computation, vol. 17, no. 3, pp. 321-344, June 2013.

[87] Y. del Valle, G. K. Venayagamoorthy, S. Mohagheghi, J. Hernandez, and R. G. Harley, "Particle swarm optimization: Basic concepts, variants and applications in power systems," IEEE Transactions on Evolutionary Computation, vol. 12, no. 2, pp. 171-195, April 2008.

[88] K. Deb, Multi-Objective Optimization Using Evolutionary Algorithms. John Wiley \& Sons, 2001, vol. 16.

[89] A. Mukhopadhyay, U. Maulik, S. Bandyopadhyay, and C. A. C. Coello, "A survey of multiobjective evolutionary algorithms for data mining: Part I," IEEE Transactions on Evolutionary Computation, vol. 18, no. 1, pp. 4-19, February 2014.

[90] A. Brabazon, M. O'Neill, and I. Dempsey, "An introduction to evolutionary computation in finance," IEEE Computational Intelligence Magazine, vol. 3, no. 4, pp. 42-55, November 2008.

[91] Z. Fei, B. Li, S. Yang, C. Xing, H. Chen, and L. Hanzo, "A survey of multiobjective optimization in wireless sensor networks: Metrics, algorithms, and open problems," IEEE Communications Surveys Tutorials, vol. 19, no. 1, pp. 550-586, September 2016.

[92] H. Takagi, "Interactive evolutionary computation: fusion of the capabilities of EC optimization and human evaluation," Proceedings of the IEEE, vol. 89, no. 9, pp. 1275-1296, September 2001.

[93] H. Takagi, T. Takahashi, and K. Aoki, "Applicability of interactive evolutionary computation to mental health measurement," in Proc. 2004 IEEE International Conference on Systems, Man and Cybernetics (IEEE Cat.), vol. 6, October 2004.

[94] H. Zhu, S. Wang, and Z. Wang, "Emotional music generation using interactive genetic algorithm," in Proc. 2008 International Conference on Computer Science and Software Engineering (CASCON), vol. 1, December 2008. 
[95] J. Kim, J. Han, Y. Kim, S. Choi, and E. Kim, "Preference-based solution selection algorithm for evolutionary multiobjective optimization," IEEE Transactions on Evolutionary Computation, vol. 16, no. 1, pp. 20-34, February 2012.

[96] K. Deb, A. Sinha, P. J. Korhonen, and J. Wallenius, "An interactive evolutionary multiobjective optimization method based on progressively approximated value functions," IEEE Transactions on Evolutionary Computation, vol. 14, no. 5, pp. 723-739, October 2010.

[97] N. Eswara, S. Ashique, A. Panchbhai, S. Chakraborty, H. P. Sethuram, K. Kuchi, A. Kumar, and S. S. Channappayya, "Streaming video QoE modeling and prediction: A long short-term memory approach," IEEE Transactions on Circuits and Systems for Video Technology, pp. 1-1, 2019.

[98] Yaochu Jin and J. Branke, "Evolutionary optimization in uncertain environments-a survey," IEEE Transactions on Evolutionary Computation, vol. 9, no. 3, pp. 303-317, June 2005.

[99] Y. Jin, M. Olhofer, and B. Sendhoff, "On evolutionary optimization with approximate fitness functions," in Proc. 2nd Annual Conference on Genetic and Evolutionary Computation (GECCO). Morgan Kaufmann Publishers Inc., 2000.

[100] J. Branke and C. Schmidt, "Faster convergence by means of fitness estimation," Soft Computing, vol. 9, no. 1, pp. 13-20, September 2005.

[101] H. Ulmer, F. Streichert, and A. Zell, "Evolution strategies assisted by Gaussian processes with improved preselection criterion," in Proc. 2003 Congress on Evolutionary Computation (CEC), vol. 1, December 2003.

[102] Yaochu Jin, M. Olhofer, and B. Sendhoff, "A framework for evolutionary optimization with approximate fitness functions," IEEE Transactions on Evolutionary Computation, vol. 6, no. 5, pp. 481-494, October 2002.

[103] L. Gräning, Y. Jin, and B. Sendhoff, "Efficient evolutionary optimization using individual-based evolution control and neural networks: A comparative study." in Proc. European Symposium on Artificial Neural Networks (ESANN), 2005. 
[104] W. Gong, A. Zhou, and Z. Cai, "A multioperator search strategy based on cheap surrogate models for evolutionary optimization," IEEE Transactions on Evolutionary Computation, vol. 19, no. 5, pp. 746-758, October 2015.

[105] I. Loshchilov, M. Schoenauer, and M. Sebag, "A mono surrogate for multiobjective optimization," in Proc. the 12th Annual Conference on Genetic and Evolutionary Computation (GECCO), 2010.

[106] B. Liu, Q. Zhang, and G. G. E. Gielen, "A Gaussian process surrogate model assisted evolutionary algorithm for medium scale expensive optimization problems," IEEE Transactions on Evolutionary Computation, vol. 18, no. 2, pp. 180-192, April 2014.

[107] O. Grøndalen, A. Zanella, K. Mahmood, M. Carpin, J. Rasool, and O. N. Østerbø, "Scheduling policies in time and frequency domains for LTE downlink channel: a performance comparison," IEEE Transactions on Vehicular Technology, vol. 66, no. 4, pp. 3345-3360, April 2017.

[108] J. Cho, Y. Wang, I. Chen, K. S. Chan, and A. Swami, "A survey on modeling and optimizing multi-objective systems," IEEE Communications Surveys Tutorials, vol. 19, no. 3, pp. 1867-1901, May 2017.

[109] K. Deb, A. Pratap, S. Agarwal, and T. Meyarivan, "A fast and elitist multiobjective genetic algorithm: NSGA-II," IEEE Transactions on Evolutionary Computation, vol. 6, no. 2, April 2002.

[110] K. Deb and H. Jain, "An evolutionary many-objective optimization algorithm using reference-point-based nondominated sorting approach, part I: Solving problems with box constraints," IEEE Transactions on Evolutionary Computation, vol. 18, no. 4, pp. 577-601, August 2014.

[111] M. Basseur and E. Zitzler, "Handling uncertainty in indicator-based multiobjective optimization," International Journal of Computational Intelligence Research, vol. 2, no. 3, pp. 255-272, 2006.

[112] E. Zitzler, M. Laumanns, and L. Thiele, "SPEA2: Improving the strength Pareto evolutionary algorithm," Eidgenössische Technische Hochschule Zürich (ETH), Institut für Technische, Tech. Rep., 2001.

[113] B. L. Miller, D. E. Goldberg et al., "Genetic algorithms, tournament selection, and the effects of noise," Complex systems, vol. 9, no. 3, pp. 193-212, 1995. 
[114] A. Zhou, B.-Y. Qu, H. Li, S.-Z. Zhao, P. N. Suganthan, and Q. Zhang, "Multiobjective evolutionary algorithms: A survey of the state of the art," Swarm and Evolutionary Computation, vol. 1, no. 1, pp. 32-49, 2011.

[115] S. Jiang, Y. Ong, J. Zhang, and L. Feng, "Consistencies and contradictions of performance metrics in multiobjective optimization," IEEE Transactions on Cybernetics, vol. 44, no. 12, pp. 2391-2404, December 2014.

[116] D. A. Van Veldhuizen and G. B. Lamont, "On measuring multiobjective evolutionary algorithm performance," in Proc. 2000 Congress on Evolutionary Computation (CECO0), July 2000.

[117] J. R. Schott, "Fault tolerant design using single and multicriteria genetic algorithm optimization." Air force Inst. of Tech. wright-patterson AFB OH, Tech. Rep., 1995.

[118] E. Zitzler and L. Thiele, "Multiobjective evolutionary algorithms: a comparative case study and the strength pareto approach," IEEE Transactions on Evolutionary Computation, vol. 3, no. 4, pp. 257-271, November 1999.

[119] H. Li and Q. Zhang, "Multiobjective optimization problems with complicated pareto sets, MOEA/D and NSGA-II," IEEE Transactions on Evolutionary Computation, vol. 13, no. 2, pp. 284-302, April 2009.

[120] E. Zitzler, L. Thiele, M. Laumanns, C. M. Fonseca, and V. G. da Fonseca, "Performance assessment of multiobjective optimizers: an analysis and review," IEEE Transactions on Evolutionary Computation, vol. 7, no. 2, pp. 117-132, April 2003.

[121] S. García, D. Molina, M. Lozano, and F. Herrera, "A study on the use of non-parametric tests for analyzing the evolutionary algorithmsâ behaviour: a case study on the cecâ2005 special session on real parameter optimization," Journal of Heuristics, vol. 15, no. 6, p. 617, May 2008.

[122] J. Hsu, Multiple Comparisons: Theory and Methods. CRC Press, 1996.

[123] G. W. Brown, "Standard Deviation, Standard Error: Which 'Standard' Should We Use?" American Journal of Diseases of Children, vol. 136, no. 10, pp. 937-941, October 1982. [Online]. Available: https://doi.org/10.1001/archpedi.1982.03970460067015. 
[124] A. Zigomitros, F. Casino, A. Solanas, and C. Patsakis, "A survey on privacy properties for data publishing of relational data," IEEE Access, vol. 8, pp. 51 071-51 099, 2020.

[125] C. A. Ardagna, M. Cremonini, E. Damiani, S. D. C. Di Vimercati, and P. Samarati, "Location privacy protection through obfuscation-based techniques," in Proc. 2007 IFIP Annual Conference on Data and Applications Security and Privacy. Springer, 2007.

[126] L. Rocher, J. M. Hendrickx, and Y.-A. De Montjoye, "Estimating the success of re-identifications in incomplete datasets using generative models," Nature Communications, vol. 10, 2019.

[127] C. Dwork, A. Roth et al., "The algorithmic foundations of differential privacy." Foundations and Trends in Theoretical Computer Science, vol. 9, no. 3-4, pp. 211-407, 2014.

[128] C. Dwork, F. McSherry, K. Nissim, and A. Smith, "Calibrating noise to sensitivity in private data analysis," in Proc. 2006 Theory of Cryptography Conference. Springer, 2006.

[129] Ú. Erlingsson, V. Feldman, I. Mironov, A. Raghunathan, K. Talwar, and A. Thakurta, "Amplification by shuffling: From local to central differential privacy via anonymity," in Proc. 2019 Thirtieth Annual ACM-SIAM Symposium on Discrete Algorithms, 2019.

[130] C. Dwork, K. Talwar, A. Thakurta, and L. Zhang, "Analyze gauss: optimal bounds for privacy-preserving principal component analysis," in Proc. 2014 forty-sixth Annual ACM Symposium on Theory of Computing, 2014.

[131] M. Abadi, A. Chu, I. Goodfellow, H. B. McMahan, I. Mironov, K. Talwar, and L. Zhang, "Deep learning with differential privacy," in Proc. 2016 ACM SIGSAC Conference on Computer and Communications Security, 2016.

[132] "TensorFlow: Large-scale machine learning on heterogeneous systems," 2015. [Online]. Available: https://www.tensorflow.org. 